Article

\title{
Composition of Modern Dust and Holocene Aeolian Sediments in Archaeological Structures of the Southern Levant
}

\author{
Bernhard Lucke ${ }^{1, *(\mathbb{C}}$, Amir Sandler ${ }^{2}$, Kim André Vanselow $^{1}{ }^{(0}$, Hendrik J. Bruins ${ }^{3}{ }^{(}$, \\ Nizar Abu-Jaber ${ }^{4}$, Rupert Bäumler ${ }^{1}$, Naomi Porat ${ }^{2}$ and Paula Kouki ${ }^{5}$ (D) \\ 1 FAU Erlangen-Nürnberg, Institute of Geography, Wetterkreuz 15, 91058 Erlangen, Germany; \\ kim.vanselow@fau.de (K.A.V.); rupert.baeumler@fau.de (R.B.) \\ 2 Geological Survey of Israel, Jerusalem 9692100, Israel; sandler@gsi.gov.il (A.S.); naomi.porat@gsi.gov.il (N.P.) \\ 3 Sede Boker Campus \& Department of Geography and Environmental Development, Ben-Gurion University \\ of the Negev, Jacob Blaustein Institutes for Desert Research, Beer-Sheva 8410501, Israel; hjbruins@bgu.ac.il \\ 4 School of Natural Resources Engineering and Management, German Jordanian University, Madaba, \\ Amman 11180, Jordan; nizar.abujaber@gju.edu.jo \\ 5 Ancient Near Eastern Empires Centre of Excellence, P.O. Box 3, University of Helsinki, \\ 00014 Helsinki, Finland; paula.kouki@hamina.fi \\ * Correspondence: bernhard.lucke@fau.de; Tel.: +49-176-4380-4256
}

Received: 24 October 2019; Accepted: 27 November 2019; Published: 30 November 2019

\begin{abstract}
Archaeological structures are often filled with sediments and may serve as effective dust traps. The physical parameters and chemical composition of archaeological soils in hilltop ruins, ancient runoff-collecting terraces, and cleanout spoils of cisterns were determined in the Petra region in southern Jordan and the Northern Negev in Israel. Different types of ruins are characterized by certain soil structures, but could not be distinguished with regard to substrate composition. This reflects a predominance of aeolian processes for primary sedimentation, while fluvial processes seem to only re-distribute aeolian material. In the Petra region, the physical and chemical properties of all archaeological soils show a significant local contribution from associated weathered rocks. Compared to modern settled dust, archaeological soils in Southern Jordan are enriched with various major and trace elements associated with clays and oxide coatings of fine silt particles. This seems connected with preferential fixation of silt and clay by surface crusts, and a role of moisture in sedimentation processes as calcareous silt was found to be deposited in greater amounts when associated with precipitation. In contrast, the contribution of rocks is negligible in the Negev due to greater rock hardness and abundant biological crusts that seal surfaces. Archaeological soils in the Negev are chemically similar to current settled dust, which consists of complex mixtures of local and remote sources, including significant portions of recycled material from paleosols. Archaeological soils are archives of Holocene dust sources and aeolian sedimentation processes, with accretion rates exceeding those of Pleistocene hilltop loess in the Negev. Comparison with Pleistocene paleosols suggests that dust sources did not change significantly, but disappearance of snow could have reduced dust accumulation during the Holocene.
\end{abstract}

Keywords: Holocene; loess; archaeological soils; dust; aeolian sedimentation; EMMAgeo; runoff-irrigated terraces; snow; precipitation influence; pre-weathered deposits

\section{Introduction}

Pleistocene loess deposits are widespread in the Central and Northern Negev desert. They are important environmental archives: loess profiles were investigated as records of dust deposition, 
accumulation, and soil development [1-12]. The Negev loess provides fertile soils for agriculture where irrigation by collected runoff or cisterns is practiced. Ancient agricultural terraces gathered runoff water and sediments and enabled cities in the Negev desert and Southern Jordan to flourish, in particular during the Roman and Byzantine period [13-15]. In Jordan, however, loess-like sediments were postulated [16,17], but have been documented only in few cases [18,19].

Holocene deposits of settling dust in the Negev are hardly recorded [20]. This has been attributed to pronounced rainfalls which lead to erosion rather than accumulation [21]. An additional cause could be diminishing of snowfall, which was assumed to be frequent during the Pleistocene $[19,22]$. Snow in the Southern Levant mostly causes minimal runoff, and could enhance dust deposition and sediment-fixing agents such as vegetation and biocrusts [19]. In addition, stronger winds during the Pleistocene (with its comparatively longer time frame than the Holocene) have been proposed to produce silt-sized particles by abrasion of mobilized sand dunes [11,23]. However, Swet et al. [24] could not identify abrasion of silt-sized particles from quartz grains during wind tunnel experiments. Therefore, it seems likely that other processes than aeolian abrasion governed dust supply, and that silt deposition may have been mainly a result of medium-range transport [25].

Variations of the dust dynamics may have played an important role for landscape changes in the Southern Levant. Reduced amounts of settling dust at the onset of the Holocene were suggested by Faersthein et al. [20] to lead to smaller sediment loads, and thus more intense runoff with stronger discharges. Changes of fluvial dynamics from sediment aggradation to incision and erosion and vice versa may thus have been less the result of rainfall variations or base-level changes, but could have been triggered by dust supply and/or the type of precipitation as either snow or rain.

During the Holocene, agricultural terraces in the Negev reduced runoff and consequently, incision and erosion diminished [26]. Sediments that accumulated behind ancient terrace walls contain a significant portion of aeolian dust [27]. Aeolian dust accumulated, as well, in the ruins of houses and other built structures $[28,29]$. This was confirmed by a recent study of soils on archaeological hilltop ruins in the Northern Negev and Petra region [19]. What could not yet be clarified, though, is whether these Holocene aeolian sediments reflect primary deposition, or re-deposition of "recycled" old dust [30] that was eroded from paleosols.

Sediments in archaeological ruins, hitherto unexplored, are potential environmental archives of dust supply and climate variations. Thus, a study of sediments in all types of man-made structures in the desert margins can assist in the reconstruction of Holocene environmental conditions, including sedimentation rates and provenance of dust. However, terraces and cisterns collected water and sediments from catchments and may thus contain substrates derived from nearby rock outcrops. This may lead to differing sediment compositions in the various types of archaeological ruins: higher shares of material derived from local rocks could be expected in profiles that received sediments from larger fluvial catchments.

In order to address the question how different types of ruins recorded dust deposition, we studied three types of archaeological structures in the Northern Negev in Israel and in the Petra region in Southern Jordan: (1) ruins of buildings on hilltops, (2) agricultural terraces, and (3) cleanout spoils from cisterns (representing sedimentation during the time of cistern operation). The studied sites were assumed to date mainly to the Roman-Nabatean till Islamic periods. In order to connect the historical sediment record to current dust in the atmosphere, dry dust collectors were placed near the study sites. The different lithologies and geomorphologies of the study areas, as well as potentially diverse atmospheric dust sources [31], allow for the role of local sediment sources and regional deposition patterns to be assessed. In order to compare Pleistocene deposits with the archaeological sediments, Late Pleistocene-Early Holocene paleosols in both study areas were also investigated. This research continues and extends the work of Lucke et al. [19], who compared sediments in archaeological hilltop ruins with current settled dust.

Our work hypotheses are as follows:

- Sediments in various archaeological structures contain a major dust component. 
- The comparison of different archaeological structures (hilltop ruins, terraces on slopes, cisterns) allows assessing the role of local processes for sediment deposition.

- Atmospheric dust sources and their potential changes over time can be identified.

- Understanding deposition processes in the archaeological structures in the context of current dust dynamics will improve our knowledge of past landscape changes.

\section{Study Areas}

\subsection{Petra Region}

The study sites in Jordan lie in the vicinity of the mountain Jabal Haroun (site of the pilgrimage sanctuary of Aaron/Haroun) near Petra. This region has been surveyed by the archeological Finnish Jabal Haroun Project (FJHP) [32-38] (Figure 1).
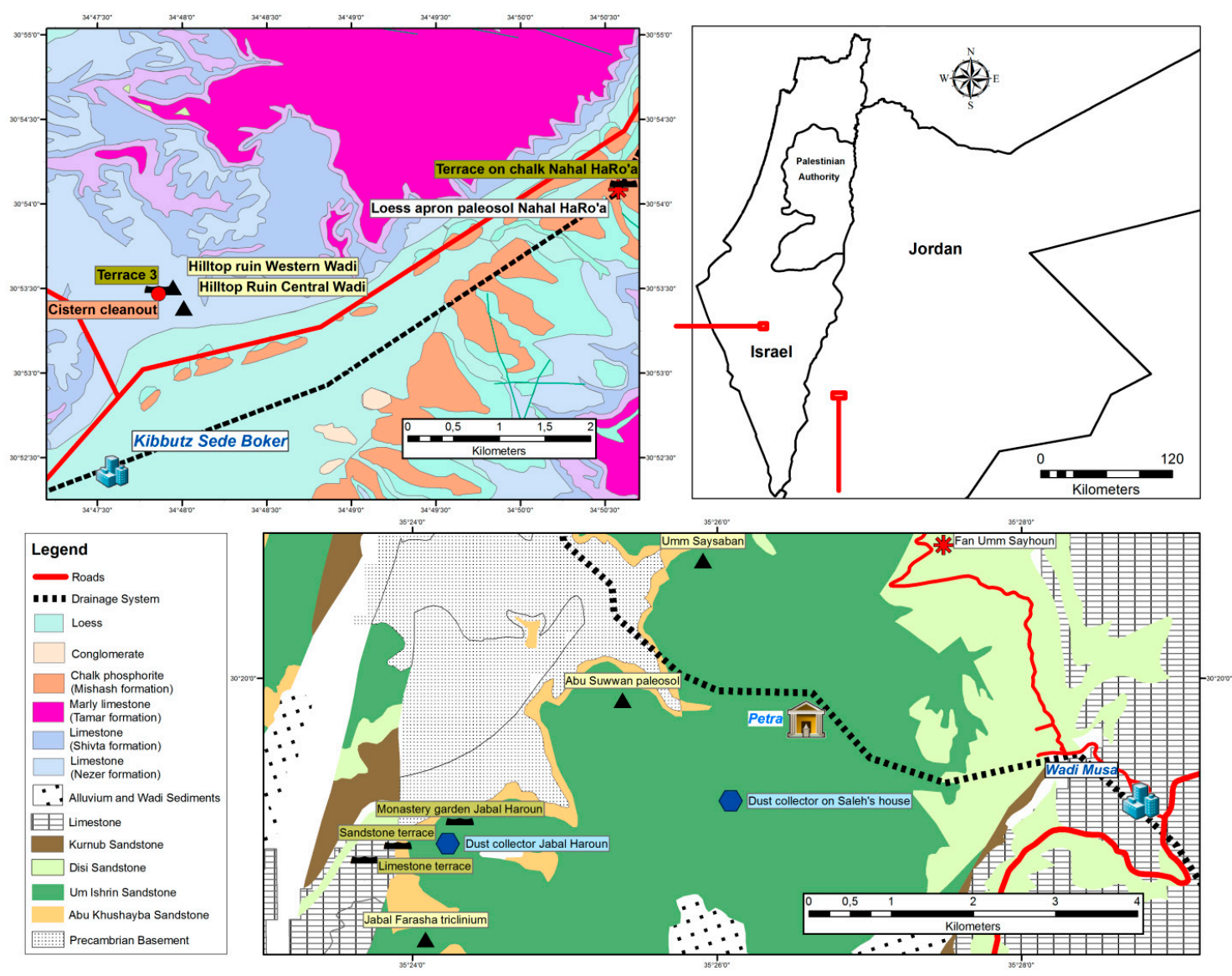

Figure 1. Map showing the locations of the study areas in Israel and Jordan and the main geological units. Black triangles mark the studied archaeological hilltop soils, black wall symbols resemble terraces, orange asterisks mark the sampled reference sites, and blue hexagons mark the dust collectors in the Petra region. The dust collector in the Negev is located c. $4 \mathrm{~km}$ to the south from the sampling sites in Midreshet Ben Gurion. Rock outcrops and reference samples not marked on the map were located close to the sampled hilltop ruins.

Mountains in the Petra region reach elevations of 900-1200 m. Cambrian continental sandstones dominate the area near Jabal Haroun [39]. The Dead Sea transform fault produced horst structures that form a highly diverse geology including patches of Turonian (partly dolomitic) limestones and Precambrian igneous rocks [39]. A significant part of the substrate of the weakly developed natural soils in the Petra region (frequently Arenosol or Regosol types, and mostly situated on alluvial deposits or other areas trapping fine particles) comprises sand derived from local fans and eroded sandstones [40]. 
In contrast, substrates of soils within archaeological hilltop ruins contain a calcareous component associated with the silt fraction, which represents long-range dust transport [19].

The arid climate of both study areas corresponds to the BWh classification of the Köppen-Geiger system [41], with rains occurring mostly from November to March. Mean annual rainfall in the Petra region is $153 \mathrm{~mm}$ (Wadi Musa weather station, 1984-2011), with high variations: $274 \mathrm{~mm}$ in the wettest season 1987/88 during the above-mentioned period, or $42 \mathrm{~mm}$ in the driest season of 2010-2011. Wind directions are dominated by western winds (see Figure 2). Wind speeds above ground during the sampling period varied mostly between $5-35 \mathrm{~km} / \mathrm{h}$, with max speeds of up to $61 \mathrm{~km} / \mathrm{h}$ [19].

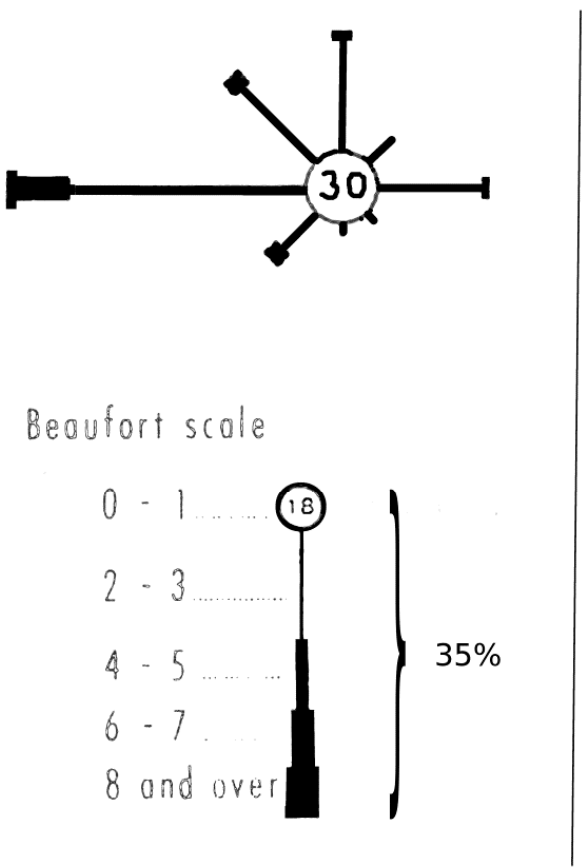

Beersheva
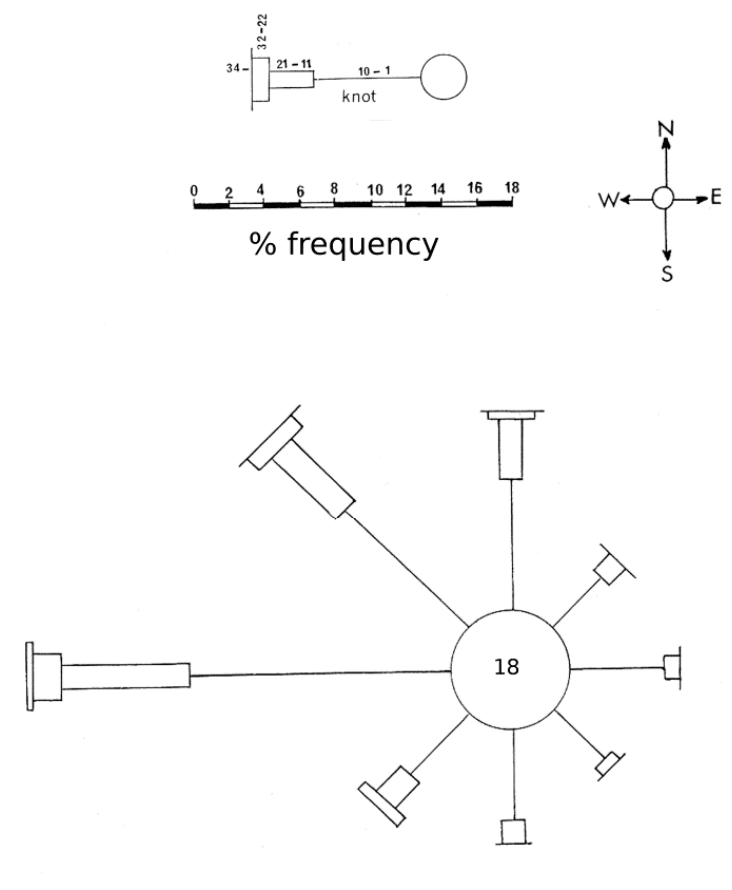

Ma'an

Figure 2. Wind diagrams showing the probabilities of wind directions and strengths near Beer Sheva according to Rosenan [42], and Ma'an according to [43] (p. 110), modified. Beersheva is located ca. $40 \mathrm{~km}$ north of Horvat Haluqim, and Ma'an $\sim 35 \mathrm{~km}$ east of Jabal Haroun. Numbers in the circles indicate the probability in \% of calm conditions. Lengths of bars correspond to probability of wind directions (see scales), and bar forms and their size to possible wind strengths and their probability (in Beaufort for Beersheva, and in Knots for Ma'an). At both locations, winds from western directions dominate.

\subsection{The Northern Negev}

The studied sites in the Northern Negev are located in and around Horvat Haluqim near Sede Boker. The ancient runoff-harvesting and respective terrace systems in this area have previously been studied [44-50]. The elevation of the study region is between 450 and $600 \mathrm{~m}$. Its main lithology is massive to well-bedded shallow marine Turonian limestone, including a continental clastic unit of sandstone and paleosols [51]. One studied site lies on an outcrop of Santonian massive chalk. Patches of Pleistocene colluvial-aeolian aprons with loessial paleosols were preserved in some areas, suggesting that the area was once covered by extensive loess blankets $[8,20]$. Substrates of the weakly developed soils (frequently Regosols) are silt-dominated.

The mean annual rainfall at Sede Boker is $93 \mathrm{~mm}$ (average for 1990-2000), but variations are high: $188 \mathrm{~mm}$ in a wet season like 1991/92, or $34 \mathrm{~mm}$ in a dry season like 1998/99. The current average P/PET-ratio (a more relevant figure for agriculture than precipitation) was determined by Bruins [46] to be 0.07 . Western winds prevail in the Northern Negev (see Figure 2). Wind speeds above ground varied mostly between 10 and $30 \mathrm{~km} / \mathrm{h}$ during dust sampling, with maximum speeds of up to $84 \mathrm{~km} / \mathrm{h}$ [19]. 


\subsection{Sampled Profiles}

A short summary of the sampling sites is presented here, and in detail in Appendix A. We studied "archaeological soils" developed in archaeological structures with parent materials probably largely derived from aeolian sediments. The term refers to material that gathered in the various structures, and includes the subgroups of "hilltop ruin soils", "terrace soils", and "cistern cleanout spoil". Soil types were classified according to WRB [52] (see Tables 2 and 3).

At Jabal Haroun in Jordan, two hilltop ruin soils were sampled along with two hilltop paleosols that were preserved below the ruins of Neolithic settlements, besides a natural hilltop soil profile on a sandstone plateau (see Figures A1 and A2 in Appendix A). These sites, as well as some rock outcrops, were described in detail by Lucke et al. [19]. In addition, one profile was excavated on a sandstone plateau next to the ruins of a Byzantine monastery, where wall remains suggested the presence of a garden during antiquity (see Figure A2 in Appendix A). Two runoff-irrigated terraces were sampled: on the central part of a dolomitic limestone slope, and on the lower part of a sandstone slope. Some outcrop rocks were analyzed, too (see Figure 1, Table 1, and Figure A2 in Appendix A).

Table 1. List of sampled profiles and collected samples. Samples were consistently numbered for statistical analysis, and assigned to deposit types that could be statistically modeled. A complete sample list with names, numbers, and deposit types is shown in Table A8 in the Appendix C.

\begin{tabular}{|c|c|c|c|}
\hline Site Name & Coordinates & Sample Numbers & Description \\
\hline \multicolumn{4}{|c|}{ Petra Region: Jabal Haroun } \\
\hline Sandplateau & $\begin{array}{l}\text { N } 30.41564 \\
\text { E } 35.46117\end{array}$ & 5 samples (no. $47-50,39$ ) & $\begin{array}{l}\text { Natural, currently forming hilltop soil on } \\
\text { sandstone plateau NW of Jabal Haroun }\end{array}$ \\
\hline $\begin{array}{l}\text { Jabal Farasha triclinium (Jf } \\
\text { site 124) }\end{array}$ & $\begin{array}{l}\text { N } 30.30445 \\
\text { E } 35.40141\end{array}$ & 4 samples (no. 67-69, 38) & $\begin{array}{l}\text { Soil covering the ruins of a triclinium on a } \\
\text { hilltop southwest of Jabal Haroun }\end{array}$ \\
\hline Umm Saysaban & $\begin{array}{l}\text { N } 30.34595 \\
\text { E } 35.43178\end{array}$ & 2 samples (no. 70-71) & $\begin{array}{l}\text { Soil covering the ruins of the hilltop site of } \\
\text { Umm Saysaban north of Jabal Haroun }\end{array}$ \\
\hline Shkarat Msaied paleosol & $\begin{array}{l}\text { N } 30.44372 \\
\text { E } 35.43917\end{array}$ & 1 sample (no. 59 ) & $\begin{array}{c}\text { Paleosol preserved below ruin of Neolithic } \\
\text { hilltop site NW of Jabal Haroun }\end{array}$ \\
\hline Abu Suwwan paleosol & $\begin{array}{l}\text { N } 30.33064 \\
\text { E } 35.42297\end{array}$ & 1 sample (no. 58$)$ & $\begin{array}{c}\text { Paleosol preserved below ruin of Neolithic } \\
\text { hilltop site NW of Jabal Haroun }\end{array}$ \\
\hline Monastery garden (Jh site 2) & $\begin{array}{l}\text { N } 30.31665 \\
\text { E } 35.40518\end{array}$ & 7 samples (no. 51-57) & $\begin{array}{c}\text { Soil in remains of a walled monastery } \\
\text { garden on sandstone plateau at Jabal } \\
\text { Haroun }\end{array}$ \\
\hline $\begin{array}{l}\text { Terrace on limestone (Jh site } \\
\qquad 60 \text { ) }\end{array}$ & $\begin{array}{l}\text { N } 30.31244 \\
\text { E } 35.39476\end{array}$ & 4 samples (no. $72-75$ ) & $\begin{array}{l}\text { Runoff-irrigated terrace on the central part } \\
\text { of a slope on dolomitic limestone }\end{array}$ \\
\hline $\begin{array}{l}\text { Terrace on sandstone (Jh site } \\
\text { 33) }\end{array}$ & $\begin{array}{l}\text { N } 30.31665 \\
\text { E } 35.40518\end{array}$ & 7 samples (no. 60-66) & $\begin{array}{l}\text { Runoff-irrigated terrace on the lower part of } \\
\text { a steep slope on sandstone }\end{array}$ \\
\hline Reference samples & $\begin{array}{l}\text { See Appendix A for } \\
\text { coordinates }\end{array}$ & 7 samples (no. 40-46) & $\begin{array}{c}\text { Various bedrock samples and geological } \\
\text { outcrops from the Petra region }\end{array}$ \\
\hline $\begin{array}{l}\text { Current dust mountain } \\
\text { summit }\end{array}$ & $\begin{array}{l}\text { N } 30.31520 \\
\text { E } 35.40406\end{array}$ & $\begin{array}{l}6 \text { samples (no. } 76-79 \\
83-84)\end{array}$ & $\begin{array}{l}\text { Samples collected on top of Jabal Haroun } \\
\text { during dust storms from } 11 / 2016-08 / 2017\end{array}$ \\
\hline $\begin{array}{l}\text { Current dust foot of } \\
\text { mountain }\end{array}$ & $\begin{array}{l}\text { N } 30.319891 \\
\text { E } 35.435040\end{array}$ & 3 samples (no. 80-82) & $\begin{array}{l}\text { Samples collected at foot of Jabal Haroun } \\
\text { during dust storms from } 11 / 2016-08 / 2017\end{array}$ \\
\hline \multicolumn{4}{|c|}{ Northern Negev: Horvat Haluqim } \\
\hline Terrace Haroa Farm & $\begin{array}{l}\text { N } 30.90111 \\
\text { E } 34.84350\end{array}$ & 6 samples (no. 7-12) & $\begin{array}{c}\text { Runoff-irrigated terrace in a tributary of } \\
\text { Nahal HaRo'a on chalk bedrock }\end{array}$ \\
\hline NH-LA & $\begin{array}{l}\text { N } 30.30140 \\
\text { E } 34.84296\end{array}$ & 2 samples (no. 5-6) & $\begin{array}{c}\text { Pleistocene loessial paleosol of a } \\
\text { colluvial-aeolian apron near Nahal HaRo'a }\end{array}$ \\
\hline HH-WW-Cistern Cleanout1 & $\begin{array}{l}\text { N } 30.89114 \\
\text { E } 34.79768\end{array}$ & 4 samples (no. 18-21) & $\begin{array}{l}\text { Ancient cleanout sediment pile next to open } \\
\text { cistern in western wadi of Horvat Haluqim }\end{array}$ \\
\hline HH-WW-R1 & $\begin{array}{l}\text { N } 30.89151 \\
\text { E } 34.79909\end{array}$ & 2 samples (no. 22-23) & $\begin{array}{l}\text { Soil covering a hilltop tumulus ruin near } \\
\text { the western wadi of Horvat Haluqim }\end{array}$ \\
\hline HH-CW-Ruin & $\begin{array}{l}\text { N } 30.88948 \\
\text { E } 34.80015\end{array}$ & 6 samples (no. 24-29) & $\begin{array}{l}\text { Soil covering a circular hilltop ruin near the } \\
\text { central wadi of Horvat Haluqim }\end{array}$ \\
\hline Terrace HH-WW-T3 & $\begin{array}{l}\text { N } 30.89069 \\
\text { E } 34.79769\end{array}$ & 5 samples (no. 13-17) & $\begin{array}{c}\text { Runoff-irrigated terrace in western wadi of } \\
\text { Horvat Haluqim on hard limestone }\end{array}$ \\
\hline Reference samples & $\begin{array}{l}\text { See Appendix A for } \\
\text { coordinates }\end{array}$ & 5 samples (no. $1-4,90$ ) & $\begin{array}{c}\text { Turonian paleosol, and various rock } \\
\text { outcrops at Horvat Haluqim }\end{array}$ \\
\hline Current dust Sede Boker & $\begin{array}{l}\text { N } 30.85135 \\
\text { E } 34.78099\end{array}$ & 8 samples (no. $30-37$ ) & $\begin{array}{l}\text { Dust samples collected at Midreshet Ben } \\
\text { Gurion in Sede Boker from 2003-2015 }\end{array}$ \\
\hline
\end{tabular}

At Horvat Haluqim in Israel, two hilltop ruin soils were sampled, along with two samples of a Pleistocene paleosol preserved in a nearby loessial apron adjacent to Nahal HaRo'a (see Figure A3 in 
Appendix A). These profiles, as well as some rock outcrops, were described in detail by Lucke et al. [19]. In addition, two runoff-irrigated agricultural terraces were sampled: on chalk in a tributary of Nahal HaRo'a, and on hard limestone in the western wadi of Horvat Haluqim. Ancient cleanout spoil of a cistern in the western wadi was also studied (see Figure 1, Table 1, and Figure A3 in Appendix A).

\subsection{Current Dust}

Plastic boxes of $45 \times 35 \mathrm{~cm}$ size filled with standard glass marbles were placed in two locations in the Petra region: one very close to the summit of Jabal Haroun (N 30.31520, E 35.40406), and another on the roof of a house (see Figure A4 in Appendix A), located at the foothill of the mountain close to the Snake Monument (N 30.319891, E 35.435040).

In the Northern Negev, dust was collected (by H. J. B.) at Midreshet Ben Gurion (N 30.85135, E 34.78099) from sheltered and elevated surfaces, at $80 \mathrm{~cm}$ height above ground level. These surfaces were cleaned before an expected dust storm, and after the storm the deposited dust was carefully collected in plastic bags.

There was no apparent connection of wind direction, speed, and total amount of sampled dust. One major dust collection event on Jabal Haroun during rather quiet conditions in August 2017 was probably connected with the occurrence of a dust devil [19].

\section{Methods}

Detailed descriptions of the methodologies of sample collection, laboratory analysis, dating, and statistical evaluation are provided in Appendix B. A short summary is given below.

Classification of soil and substrate followed the World Reference Base of Soil Resources (WRB) [52] and horizons description followed Soil Survey Staff [53].

Color, $\mathrm{pH}$, electrical conductivity, organic matter, bulk density, and contents of calcium carbonate were determined according to standard methods. Chemical composition of major and trace elements was determined by energy-dispersive X-ray fluorescence (Spectro XEPOS device, error less than $0.9 \%$ for major elements and $5 \%$ for trace elements). Dissolved iron was extracted with oxalate and dithionite. Mass-specific magnetic susceptibilities were measured with an Agico MFK1-FA multi-function Kappabridge.

Grain sizes were determined by wet sieving and a Sedigraph grain size analyzer, without removal of $\mathrm{CaCO}_{3}$. Samples of current dust were often too small and thus determined with a Malvern MasterSizer 3000 laser diffraction analyzer, applying ultrasonic for aggregate dispersal and a clay-silt border of $5 \mu \mathrm{m}$ [11]. Comparability of the two methods was satisfactory for dust samples [19] (pp. 50-53). For selected samples from the Petra region, sand was removed by sieving and the remaining silt and clay fractions analyzed for their element contents in order to check whether they were more similar to silt-rich dust deposited with precipitation.

Sediments were dated by the Optically Stimulated Luminescence (OSL) method and by the ${ }^{14} \mathrm{C}$ method for organic material. Archaeological ages were derived from artifact and building styles.

We used the Gradistat for Excel program for calculating grain size statistics [54] and the EMMAgeo v0.9.4 R package for grain size end-member analysis [55]. In addition, we applied Principle Component Analysis (PCA) using the R-function prcomp [56] to identify similarities between samples in the multivariate space. In order to explore the suitability and importance of selected parameters for distinguishing between different types of deposits, we applied random forest classification using the R-package randomForest [57]. As well, simple linear regressions were used for evaluating connections of selected parameters. 


\section{Results}

\subsection{General Properties of Archaeological Soils}

\subsubsection{Petra Region}

Schematic drawings of the profiles including soil horizons and substrates are presented in Figure 3.

General soil properties, soil types, soil horizons, and calibrated ages of selected archaeological soils and reference samples around Petra are summarized in Table 2. Full results are presented in Table A1 in Appendix C.

Natural Protic Arenosols (Ochric) are present on sandstone plateaus (Sandplateau samples). A slightly darker color than that of the disintegrated bedrock is discernible (sample Sandplateau Stein is the underlying bedrock). Calcaric Leptosols are present in hilltop ruins and Protic Colluvic Calcaric Regosol profiles are present on terraces. The Late Pleistocene-Early Holocene paleosols are Cambic Calcisols and Cambisols. Archaeological soils have browner colors of 7.5YR and 5YR, but these are still in the range of the color variations of the sandstones in the area, which range from 2.5-10 YR [58]. Soil pH values are alkaline (8-9). Total organic carbon (TOC) values are all below $1 \%$. Conductivities are mostly lower than $250 \mu \mathrm{S} / \mathrm{cm}$.

\subsubsection{The Northern Negev}

Schematic drawings of the profiles, including soil horizons and substrates, are presented in Figure 4.

General soil properties, soil types, soil horizons, and calibrated ages of selected archaeological soils and reference samples are summarized in Table 3. Full results can be found in Table A2 at Appendix C.

Calcaric Leptosols and Calcaric Regosols are present in the hilltop ruins. Protic Colluvic Calcaric Regosols occur in the terraces, while the profile in the cistern spoil shows a Protic Calcaric Regosol. Soil colors are yellow or pale brown, all in the range of 10YR. The reference samples of the rock outcrops, in contrast, range from 2.5YR to 7.5YR. The paleosols below ruins, and of loessial aprons, are Cambic Calcisols.

pH-values are in the alkaline milieu of 8-9. Most TOC values are below 1\%, but slightly elevated values of 1.1-1.4\% were determined in some hilltop ruin soils and in the Apb-horizon of the runoff-irrigated terrace on chalk in a tributary of Nahal HaRo'a. The topsoil of the latter has a high value of $3 \%$ TOC that reflects the dense current vegetation at the terrace wall, which apparently still stores water.

Conductivities vary from relatively low values of 107-265 $\mu \mathrm{S} / \mathrm{cm}$ in the loessial paleosol and in Terrace 3 in the western wadi of Horvat Haluqim, which is located rather upstream, to 4520-4960 $\mu \mathrm{S} / \mathrm{cm}$ in the terrace sediments in the tributary of Nahal HaRo'a, which is located rather downstream. The variability seems related to the geomorphology and geology, and partly to rainwater storing capacity of archeological soils within the wall remains of the hilltop ruins. The low sand fraction of the Northern Negev soils, compared with the Petra region, causes reduced drainage and higher salt accumulation. 
Table 2. Soil classifications, diagnostic horizons, available ages, color, $\mathrm{pH}$, conductivities (EC), and total organic carbon (TOC) of selected samples of the Petra region. All results are presented Table A2 in Appendix C. The sample no. reflects the sample's number used in statistical analyses, ordered according to types of deposits (see Table A8 in the Appendix C).

\begin{tabular}{|c|c|c|c|c|c|c|c|c|c|c|}
\hline Sample Name & Sample No. & $\begin{array}{l}\text { Horizon from } \\
\ldots \text { to } \ldots(\mathrm{cm})\end{array}$ & $\begin{array}{l}\text { Sampling } \\
\text { Depth }(\mathrm{cm})\end{array}$ & $\begin{array}{l}\text { Calibrated }{ }^{14} \mathrm{C} \\
\text { Age (1-Sigma) }\end{array}$ & $\begin{array}{l}\text { Cal. OSL } \\
\text { Age }\end{array}$ & Age from Context & Munsell Dry & $\mathrm{pH}\left(\mathrm{H}_{2} \mathrm{O}\right)$ & $\mathrm{EC}(\mu \mathrm{S} / \mathrm{cm})$ & TOC \% \\
\hline \multicolumn{11}{|c|}{ Natural Hilltop Soil on Sandstone Plateau: Protic Arenosol (Ochric) } \\
\hline Sandplateau 1 & 47 & $\mathrm{~A}(0-10)$ & 10 & & & & 10 YR 6/4 1. y. brown & 8.4 & 66 & 0.28 \\
\hline Sandplateau 2 & 48 & C (10-70) & 30 & & & & 10 YR 5/6 y. brown & 8.5 & 60 & 0.16 \\
\hline Sandplateau Stein & 39 & $\mathrm{R}(70+)$ & bedrock & & & & $10 \mathrm{YR} 8 / 1$ white & 8.5 & 52 & 0.03 \\
\hline \multicolumn{11}{|c|}{ Jabal Farasha Triclinium Hilltop Ruin: Calcaric Leptosol (Protic) } \\
\hline JF site $124 / 15 \mathrm{~cm}$ & 67 & $\mathrm{~V}(0-10)$ & 5 & \multirow{4}{*}{\multicolumn{2}{|c|}{ 880-1040 CE }} & Nabatean pottery & $7.5 Y R 5 / 3$ brown & 8.5 & 112 & 0.94 \\
\hline $\mathrm{JF}$ site $124 / 115 \mathrm{~cm}$ & 68 & C (10-25) & 15 & & & \multirow{3}{*}{$\sim 200 \mathrm{CE}$} & 7.5YR 5/4 brown & 8.6 & 86 & 0.53 \\
\hline JF site $124 / 125 \mathrm{~cm}$ & 69 & C (10-25) & 25 & & & & 5YR 5/4 r. brown & 8.5 & 79 & 0.52 \\
\hline JF site 124/1 rock & 38 & $\mathrm{R}(25+)$ & bedrock & & & & 10R 5/4 weak red & & & 0.13 \\
\hline \multicolumn{11}{|c|}{ Umm Saysaban Hilltop Ruin: Calcaric Leptosol (Protic) } \\
\hline Umm Saysaban $5 \mathrm{~cm}$ & 70 & $\mathrm{~V}(0-5)$ & 5 & & & & 7.5YR 6/3 light brown & 8.5 & 106 & 0.58 \\
\hline Umm Saysaban $10 \mathrm{~cm}$ & 71 & $C(5-10)$ & 10 & & & $\sim 2500 \mathrm{BCE}$ & $10 Y R 7 / 3$ v. p. brown & 8.5 & 83 & 0.38 \\
\hline \multicolumn{11}{|c|}{ Buried Early Holocene Paleosols: Cambic Calcisol (Hypocalcic) (below Shkarat Msaied Ruins); Cambisol (Protocalcic) (below Abu Suwwan Ruins) } \\
\hline $\begin{array}{l}\text { Shkarat Msaied 1(paleosol } \\
\text { below ruin) }\end{array}$ & 59 & $3 \mathrm{BCk}(45-60+)$ & 55 & & & $>8000 \mathrm{BCE}$ & 7.5 YR 7/3 pink & 8.3 & 1375 & 0.27 \\
\hline $\begin{array}{l}\text { Abu Suwwan below nw } 65 \\
\text { (buried paleosol) }\end{array}$ & 58 & ЗВС $(60-70+)$ & 65 & & & $>8000 \mathrm{BCE}$ & 10 YR 5/2 g. brown & 8.1 & 142 & 0.60 \\
\hline \multicolumn{11}{|c|}{ Monastery Garden of Jabal Haroun: Protic Arenosol (Alcalic. Ochric) } \\
\hline Jh Site $210 \mathrm{~cm}$ & 51 & A $(0-10)$ & 10 & & \multirow{4}{*}{$\begin{array}{c}150 \text { BCE }-90 \\
\text { CE }\end{array}$} & \multirow[t]{3}{*}{ Byz-Um. pottery } & 7.5YR 6/4 light brown & 8.5 & 224 & 0.27 \\
\hline Jh Site $250 \mathrm{~cm}$ & 55 & $2 \mathrm{Cu}(35-60)$ & 50 & 239-327 CE & & & 7.5YR 5/3 brown & 8.8 & 208 & 0.22 \\
\hline Jh Site $260 \mathrm{~cm}$ & 56 & $2 \mathrm{Cu}(35-60)$ & 60 & & & & 5YR 5/4 r. brown & 8.5 & 113 & 0.09 \\
\hline Jh Site $270 \mathrm{~cm}$ & 57 & $3 \mathrm{Cu}(60-70)$ & 70 & & & $\begin{array}{l}\text { Late Roman } \\
\text { pottery }\end{array}$ & 5YR 5/3 r. brown & 8.7 & 200 & 0.16 \\
\hline \multicolumn{11}{|c|}{ Terrace on Dolomitic Limestone (Jh site 60): Protic Colluvic Calcaric Regosol } \\
\hline Jh limestone 0 & 72 & Ap $(0-10)$ & surface & & \multirow{2}{*}{\multicolumn{2}{|c|}{$\begin{array}{l}340 \text { BCE-50 Nabatean pottery } \\
\text { CE }\end{array}$}} & $10 Y R$ 7/4 v. p. brown & 8.8 & 228 & 0.70 \\
\hline Jh limestone 60 & 75 & C (10-60) & 60 & & & & 10YR 6/4 1. y. brown & 8.8 & 518 & 0.49 \\
\hline \multicolumn{11}{|c|}{ Terrace on Sandstone (Jh site 33): Protic Calcaric Arenosol (Colluvic) over Protic Colluvic Calcaric Regosol } \\
\hline Jh site 3310 & 60 & Ap $(0-20)$ & 10 & & & \multirow[t]{4}{*}{$\begin{array}{l}\text { Nababatean - Late } \\
\text { Islamic pottery }\end{array}$} & 7.5YR 6/4 light brown & 8.8 & 239 & 0.26 \\
\hline Jh site 3330 & 61 & C (20-70) & 30 & & & & 5YR 6/4 1. r. brown & 8.4 & 363 & 0.02 \\
\hline Jh site 33100 & 64 & $2 \mathrm{C}(70-140)$ & 100 & & & & 7.5YR 6/4 1. brown & 8.8 & 688 & 0.08 \\
\hline Jh site 33150 & 66 & 3C (140-150) & 150 & & & & 5YR 6/4 1. r. brown & 8.9 & 599 & 0.10 \\
\hline \multicolumn{11}{|c|}{ Reference Samples from Petra Region: Rocks \& Current Fans } \\
\hline JH limestone outcrop & 44 & & rock outcrop & & & & 7.5YR 8/4 pink & & & 0.85 \\
\hline Beidha Fan & 46 & & surface & & & & 10YR 6/4 1. y. brown & 8.44 & 346 & 0.46 \\
\hline \multirow{2}{*}{$\begin{array}{l}\text { Fan Umm Sayhoun } \\
\text { JH sandstone (Abu } \\
\text { Khushayba) }\end{array}$} & 45 & & surface & & & & $10 Y R 8 / 4$ v. p. brown & 8.98 & 136 & 0.07 \\
\hline & 41 & & rock outcrop & & & & $2.5 Y R$ 5/3 r. brown & 8.7 & 97 & 0.02 \\
\hline
\end{tabular}




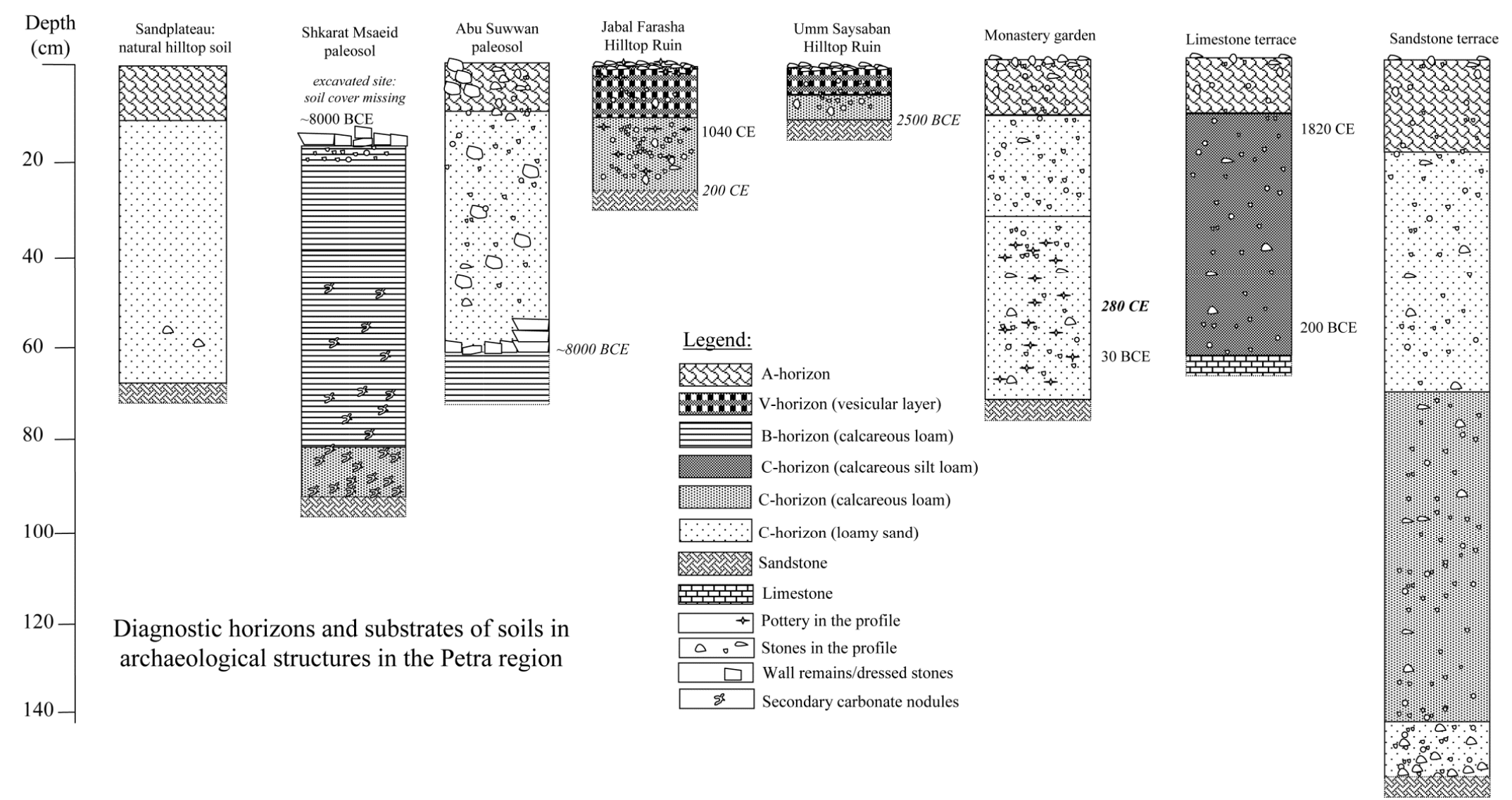

Figure 3. Schematic drawings of the profiles in the Petra region showing diagnostic horizons and substrates, along with available ages (OSL-ages in normal letters, ages from archaeological context in italics, and ${ }^{14} \mathrm{C}$-ages in bold and italics). Detailed descriptions and soil classification are displayed in Table 2, Table A1 at Appendix C, and at Appendix A.1. Full dating results are shown in Tables A3 and A4 in Appendix C. 
Table 3. Soil classifications, diagnostic horizons, available ages, color, $\mathrm{pH}$, conductivities, and total organic carbon (TOC) of selected samples of the Northern Negev. All results are presented Table A2 in Appendix C. The sample no. reflects the sample's number used in statistical analyses, ordered according to types of deposits (see Table A8 in the Appendix C).

\begin{tabular}{|c|c|c|c|c|c|c|c|c|c|c|}
\hline Sample Name & Sample No. & $\begin{array}{l}\text { Horizon from } \\
\ldots \text { to } \ldots(\mathrm{cm})\end{array}$ & $\begin{array}{c}\text { Sampling } \\
\text { Depth }(\mathrm{cm})\end{array}$ & $\begin{array}{l}\text { Calibrated }{ }^{14} \mathrm{C} \\
\text { Age (1-Sigma) }\end{array}$ & $\begin{array}{l}\text { Cal. OSL } \\
\text { Age }\end{array}$ & Age from Context & Munsell Dry & $\mathrm{pH}\left(\mathrm{H}_{2} \mathrm{O}\right)$ & $\begin{array}{c}\text { Conductivity } \\
(\mu \mathrm{S} / \mathrm{cm})\end{array}$ & тос $\%$ \\
\hline \multicolumn{11}{|c|}{ Terrace on Chalk in Nahal HaRo'a: Protic Colluvic Calcaric Regosol (Siltic) over Calcaric Fluvisol (Aric, Siltic) over Cambic Calcisol (Siltic) } \\
\hline Haroa Farm $10 \mathrm{C}$ & 7 & $\begin{array}{l}\mathrm{A}(0-5), \mathrm{C}(5-15), \\
2 \mathrm{C}(15-28)\end{array}$ & 10 & & & & 10 YR 6/4 light yellowish & 8.1 & 4520 & 3.02 \\
\hline Haroa Farm $28 \mathrm{fAh}$ & 8 & $3 \mathrm{Apb}(28-30)$ & 28 & & & & 10 YR 7/4 very pale brown & 7.8 & 4810 & 1.43 \\
\hline Haroa Farm 40 C & 9 & $3 \mathrm{C}(30-55)$ & 40 & & & & 10 YR $7 / 4$ very pale brown & 8.1 & 4960 & 0.65 \\
\hline \multicolumn{11}{|c|}{ Paleosol in Loessial Apron of tributary of Nahal HaRo'a: Cambic Calcisol (Siltic) } \\
\hline NH-LA-10 cm & 5 & $\mathrm{~V}(0-25)$ & 10 & & & & 10YR 8/6 yellow & 8.9 & 111 & 0.43 \\
\hline NH-LA-30 cm & 6 & 2Bwk (25-55+) & 30 & & & & 10YR 6/6 brownish yellow & 9.3 & 135 & 0.38 \\
\hline \multicolumn{11}{|c|}{ Cistern Cleanout Spoil: Protic Calcaric Regosol (Siltic, Transportic) over Calcaric Regosol (Siltic, Transportic) over Camic Calcisol (Siltic) } \\
\hline HH-WW-Cistern1-Cleanout1-160 & 21 & $2 \mathrm{Cu}(80-160)$ & 160 & & $590-730 \mathrm{CE}$ & $\begin{array}{l}\text { Byzantine-Islamic } \\
\text { pottery }\end{array}$ & 10 YR 7/4 very pale brown & 8.0 & 1445 & 0.38 \\
\hline \multicolumn{11}{|c|}{ Hilltop Ruin Overlooking the Western Wadi: Calcaric Leptosol (Protic, Siltic) } \\
\hline HH-WW-R1 20 & 23 & $C(5-20)$ & 20 & & & 2000-2500 BCE & 10YR 5/6 yellowish brown & 8.3 & 3890 & 1.30 \\
\hline \multicolumn{11}{|c|}{ Hilltop Ruin Overlooking the Central Wadi: Protic Calcaric Regosol (Siltic) over Cambic Calcisol (Siltic) } \\
\hline HH-CW-Ruin 25 & 25 & V2 (15-35) & 25 & $\begin{array}{c}1694-1918 \text { CE }(20 \\
\mathrm{cm})\end{array}$ & $870-1020 \mathrm{CE}$ & & 10YR $7 / 3$ very pale brown & 8.4 & 2100 & 0.84 \\
\hline HH-CW-Ruin 50 & 26 & C (40-70) & 50 & & $\begin{array}{c}\text { 2570-1990 } \\
\text { BCE }\end{array}$ & & $10 Y R$ 7/3 very pale brown & 8.7 & 3450 & 0.76 \\
\hline HH-CW-Ruin 90 & 29 & 2Bwk (70-100+) & 90 & & $\begin{array}{c}23900-20600 \\
\text { BCE }\end{array}$ & & $10 Y R \quad 7 / 4$ very pale brown & 9.0 & 3120 & 1.10 \\
\hline HH-WW-T3-20 cm & 13 & $\mathrm{~A}(0-5), \mathrm{C}(0-60)$ & 20 & & & & 10YR 7/4 Very pale Brown & 8.8 & 265 & 0.60 \\
\hline HH-WW-T3-70 cm & 15 & $2 \mathrm{C}(60-120)$ & 70 & & $320-480 \mathrm{CE}$ & & 10YR 7/4 Very pale Brown & 8.5 & 107 & 0.32 \\
\hline HH-WW-T3-120 & 17 & $2 C(60-120)$ & 120 & & $460-240 \mathrm{BCE}$ & & $10 Y R \quad 7 / 3$ very pale brown & 8.0 & 127 & 0.24 \\
\hline \multicolumn{11}{|c|}{ Negev reference samples } \\
\hline Haroa Farm chalk & 4 & chalk & outcrop & & & & 5YR $8 / 2$ pinkish white & & & \\
\hline HH-WW-C2-soft limestone & 2 & soft limestone & outcrop & & & & 5YR $8 / 1$ white & & & \\
\hline HH-CW-chalk & 3 & chalk & outcrop & & & & 7.5YR $8 / 3$ pink & & & \\
\hline
\end{tabular}



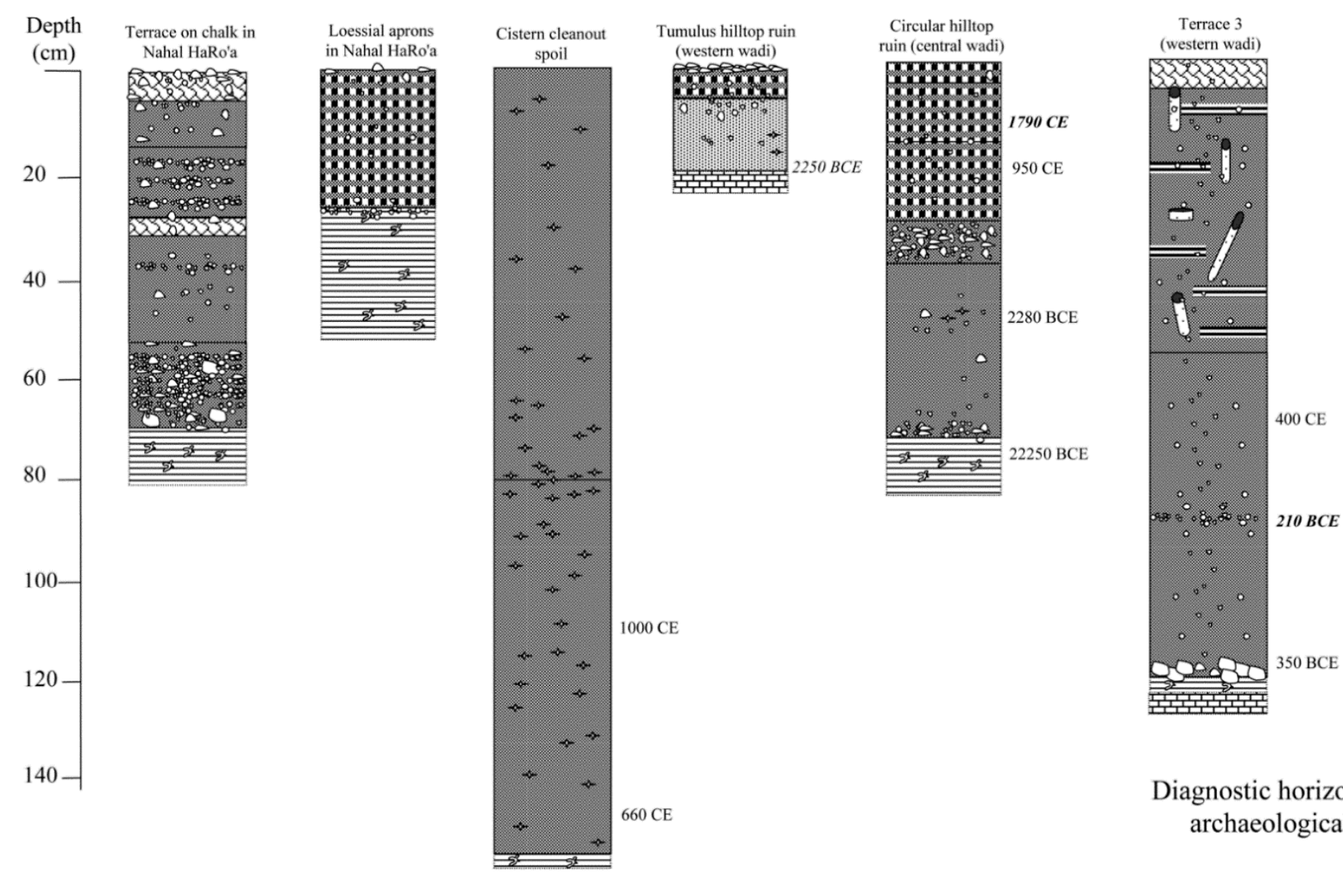

Legend:

अस्य A-horizon

2.7.7. V-horizon (vesicular layer)

B-horizon (calcareous silt loam)

C-horizon (calcareous silt loam)

\section{吕}

Filled animal burrows

(Bioturbation: Krotowinas)

Banded layers

$\$$ Secondary carbonate nodules

$\rightarrow$ Pottery in the profile

0.0 Stones in the profile

\section{Diagnostic horizons and substrates of soils in} archaeological structures in the Negev

Figure 4. Schematic drawings of the profiles in the Northern Negev showing diagnostic horizons and substrates, along with available ages (OSL-ages in normal

letters, ages from archaeological context in italics, and ${ }^{14} \mathrm{C}$-ages in bold and italics). Detailed descriptions and soil classification are displayed in Table 3 , Table A2 at Appendix C, and at Appendix A.2. Full dating results are shown in Tables A3 and A4 in Appendix C. 


\subsubsection{Comparison of Archaeological Soils in Various Structures and Substrates}

Table 4 summarizes a comparison of the basic structures and substrates of the soils in the different archaeological ruin types in both investigation regions, as well as the natural reference soils, and the substrates of the current dust samples (see also Figures 3 and 4).

Table 4. Summary of soil structures and substrates in the Negev and Petra region (except monastery garden on Jabal Haroun), and substrates of current dust samples.

\begin{tabular}{|c|c|c|c|c|}
\hline \multirow[b]{2}{*}{ Soil Type } & \multicolumn{2}{|c|}{ Petra Region } & \multicolumn{2}{|c|}{ Negev } \\
\hline & Structure & Substrate & Structure & Substrate \\
\hline Hilltop ruins & $\begin{array}{l}\text { Crust and clast } \\
\text { cover, V-C profile }\end{array}$ & $\begin{array}{l}\text { Calcareous loam, } \\
\text { occasional stones }\end{array}$ & $\begin{array}{l}\text { Crust and clast cover, } \\
\text { V-C profile }\end{array}$ & $\begin{array}{c}\text { Calcareous silt loam, } \\
\text { occasional stones }\end{array}$ \\
\hline Runoff terraces & $\begin{array}{l}\text { Compact and } \\
\text { homogeneous, or } \\
\text { sandy layers }\end{array}$ & $\begin{array}{l}\text { Calcareous (silt) } \\
\text { loam, or loamy } \\
\text { sand, occasional } \\
\text { stones }\end{array}$ & $\begin{array}{l}\text { Compact and } \\
\text { homogeneous, or } \\
\text { banded layers }\end{array}$ & $\begin{array}{c}\text { Calcareous silt loam, } \\
\text { occasional stones }\end{array}$ \\
\hline Cistern cleanout & n.a. & n.a. & $\begin{array}{l}\text { Excavated aggregates } \\
\text { of silt loam }\end{array}$ & $\begin{array}{l}\text { Calcareous silt loam, } \\
\text { free of stones }\end{array}$ \\
\hline $\begin{array}{l}\text { Natural reference } \\
\text { soils }\end{array}$ & $\begin{array}{c}\text { A-C profile } \\
\text { (sandstone hilltop) }\end{array}$ & Loamy sand & $\begin{array}{l}\text { V-2Bwk profile } \\
\text { (colluvial apron) }\end{array}$ & Calcareous silt loam \\
\hline Current dust & - & $\begin{array}{c}\text { Loamy sand } \\
\text { (dry deposition) } \\
\text { Calcareous silt } \\
\text { loam (wet } \\
\text { deposition) }\end{array}$ & - & $\begin{array}{l}\text { Calcareous silt loam } \\
\text { (dry deposition) } \\
\text { Calcareous silt } \\
\text { (wet deposition) }\end{array}$ \\
\hline
\end{tabular}

The characteristics of the studied profiles partly depend on the types of the archaeological structures. All hilltop ruin soils exhibit a vesicular layer/V-horizon below a crust and clast pavement, which suggests that their substrates are largely aeolian sediments [19]. In contrast, V-horizons could not be discerned in terrace and cistern sediments.

A large part of the terrace profiles in both study areas is homogeneous and compact. This could result of mixing by effects of plowing. The upper parts of Terrace 3 and of the terrace in the tributary of Nahal HaRo'a in the Negev have finely banded layers, apparently resulting from sorting and upward fining during fluvial processes, and probably deposited during phases without agricultural practices. However, no vesicular layers could be observed there, too, suggesting that direct aeolian sedimentation played a subordinate role in the terraces.

Banded sediments could not be observed in the Petra region. A friable, sandy layer poor in $\mathrm{CaCO}_{3}$ is present in the upper part of the sandstone terrace profile, in contrast with the compact, homogeneous calcareous loam that dominates most terrace profiles. This could indicate that an increasing contribution from weathering sandstones was deposited in this profile, while continuous plowing prevented the formation of banded layers.

Remains of soil aggregates from the excavation during cistern cleaning were identified in the cistern cleanout spoil. The profile did not show layers or bedding structures related to sedimentation in water, but it is possible that such laminations can only be discerned by micromorphology [59].

Apart from these differences, the archaeological soils of the various structures are strikingly similar. Although higher silt contents are evident in the Northern Negev, all profiles are dominated by calcareous silt, most likely of aeolian origin [19]. This is supported by the characteristics of current dust samples deposited with precipitation in the Petra region, which consist of calcareous silt loam.

An exception is the profile in the assumed monastery garden, which consists of homogeneous loamy sand with low $\mathrm{CaCO}_{3}$, and is similar to the natural reference soil on the sandstone plateau. The catchment area for potential runoff collection in the possible garden is very small, but it may have been irrigated from nearby large cisterns. Weathered sandstone rocks surrounding the plateau could have provided a significant aeolian sand contribution. Weathering of the underlying sandstone bedrock is 
unlikely as the pottery, organic remains, and available ages of the profile suggest a sedimentary origin of the substrate.

\subsection{Sediment Ages and Sedimentation Rates}

Tables 2 and 3 and Figures 3 and 4 summarize the available ages of the profiles. Details of the dating results are presented in Tables A3 and A4 in the Appendix C. Ages of the studied ruins in the Petra region show that sediments accumulated mainly during the past 2000 years. Exceptions are the hilltop ruin of Umm Saysaban, which started collecting sediments approximately 4500 years ago, and the paleosols below the Neolithic sites, which are more than 10,000 years old.

Similar ages are indicated in the Northern Negev. The sediments of Terrace 3 were deposited during the last 2300 years, while the hilltop ruins collected material since approximately 4300 years. The cistern cleanout spoil is dated to the last 1300 years, and the late Pleistocene paleosol of the loessial apron to $\sim 24,000$ years.

Average sedimentation rates in the hilltop ruins were recently calculated [19] (pp. 12-15). Sedimentation rates in other structures were calculated in the current study (Table 5). Here, we applied a revised bulk density of hilltop ruins soils, subtracting the soil skeleton $(>2 \mathrm{~mm})$, and using more conservative estimates of annual dust deposition, presented in Appendix B. The revision was applied to the previous estimates [19], as well. Accordingly, a minimum of $\sim 250 \mathrm{~g} \mathrm{~m}^{2} \mathrm{a}^{-1}$ dust was trapped by each of our standard marble dust collectors on Jabal Haroun. In comparison, the average sedimentation rate in the hilltop ruin of the triclinium on Jabal Farasha is $\sim 125 \mathrm{~g} \mathrm{~m}^{2} \mathrm{a}^{-1}$. A detailed calculation of sedimentation rates is presented in Appendix D.

Table 5. Average sedimentation rates calculated for the archaeological soils in terraces and the cistern cleanout. The following experimentally determined bulk densities were used: $0.901 \mathrm{~g} / \mathrm{cm}^{3}$ (hilltop ruins), 1.036/0.895 g/ $/ \mathrm{cm}^{3}$ (compact/friable terrace sediments), 1.296/0.954 g/ $/ \mathrm{cm}^{3}$ (compact/friable cistern cleanout spoil). Full results including re-calculated sedimentation rates in hilltop ruins are presented in Appendix C, Table A5.

\begin{tabular}{|c|c|c|c|}
\hline Site & $\mathrm{mm} /$ year & $\mathrm{g} / \mathrm{m}^{2}$ year $^{-1}$ & Comment \\
\hline \multicolumn{4}{|c|}{ Petra Region } \\
\hline Monastery garden (Jh site 2) & 0.29 & 264 & $\begin{array}{c}\text { Steady and continuous } \\
\text { sedimentation, apparently ongoing } \\
\text { Strongly scattering OSL-ages, } \\
\text { samples may consist of mixtures of } \\
\text { older and younger material }\end{array}$ \\
\hline \multicolumn{4}{|c|}{ Negev } \\
\hline Cistern Cleanout & 1.47 & 1906 & $\begin{array}{l}\text { Lower, compact part of cleanout pile } \\
\text { that was dated by OSL }\end{array}$ \\
\hline Terrace 3 & 0.51 & $453-525$ & $\begin{array}{c}\text { Possibly inhomogeneous } \\
\text { sedimentation rates }\end{array}$ \\
\hline
\end{tabular}

The estimated annual sedimentation rates suggest that deposition on the monastery garden was nearly identical to the amounts that could be collected during one year of continuous sampling with the dust collectors on Jabal Haroun. It is slightly higher than sedimentation in the limestone terrace, and about the double of the deposition rate that was calculated for the hilltop ruin of the triclinium on Jabal Farasha [19]. The cistern cleanout spoil is hardly comparable to the other archives and off-scale, while Terrace 3 collected about 3.5 times the amount that was deposited in the adjacent Negev hilltop ruins [19]. 


\subsection{Grain Sizes and $\mathrm{CaCO}_{3}$-Contents}

\subsubsection{The Petra Region}

Table 6 presents grain sizes of selected samples in the Petra region. All results are displayed in Table A6 in Appendix C. Grain sizes within each profile are rather similar, with an exception of the sandstone terrace, where grain sizes mirror the layer change to sandy substrate in its upper part. Dominance of medium sand fraction with a main mode of $\sim 415 \mu \mathrm{m}$ indicates a significant contribution by weathered sandstones, as the latter were found to consist mostly of medium sand $[19,58]$. Its contents are high in the natural soil on the sandstone plateau, and in the monastery garden, but are low in the hilltop ruins and terraces, where fine sand dominates with a main mode of $\sim 132 \mu \mathrm{m}$.

It is notable that the sandy layer within the profile of the terrace on sandstone is dominated by fine sand, along with elevated amounts of medium sand. These fractions are nearly absent from the silt-dominated layers, suggesting limited sand deposition from the surrounding rocks during formation of the silt-rich layers. In addition, coarse fractions such as skeleton $(>2 \mathrm{~mm})$ and coarse sand in the terrace sediments are not higher than in other archaeological soils, and lower than in the current alluvial fans. This suggests that runoff processes did not lead to the mobilization and deposition of coarse grains, and played a subordinate role for the composition of the sediments.

The clay fraction of the probably once cultivated soils in the terraces is higher than those of the other profiles. Terrace soils have 25-39\% clay, whereas most other samples rarely exceed contents of $15 \%$. It is worth noting that a similar pattern, although less pronounced, is evident in the soil of the monastery garden, which probably consists of sand derived from the surrounding rocks. The clay content at the lower part of the profile, which was probably subject to irrigated cultivation, is $~ 17 \%$, whereas it is only $\sim 9 \%$ in the upper part, which was subject to limited, or no cultivation.

Calcium carbonate contents show a positive correlation with the silt fraction $\left(R^{2}=0.44\right)$ (Figure 5), supporting $\mathrm{CaCO}_{3}$ and silt in the Petra region to be of aeolian origin, mainly of intermediate to long-range transport [19].

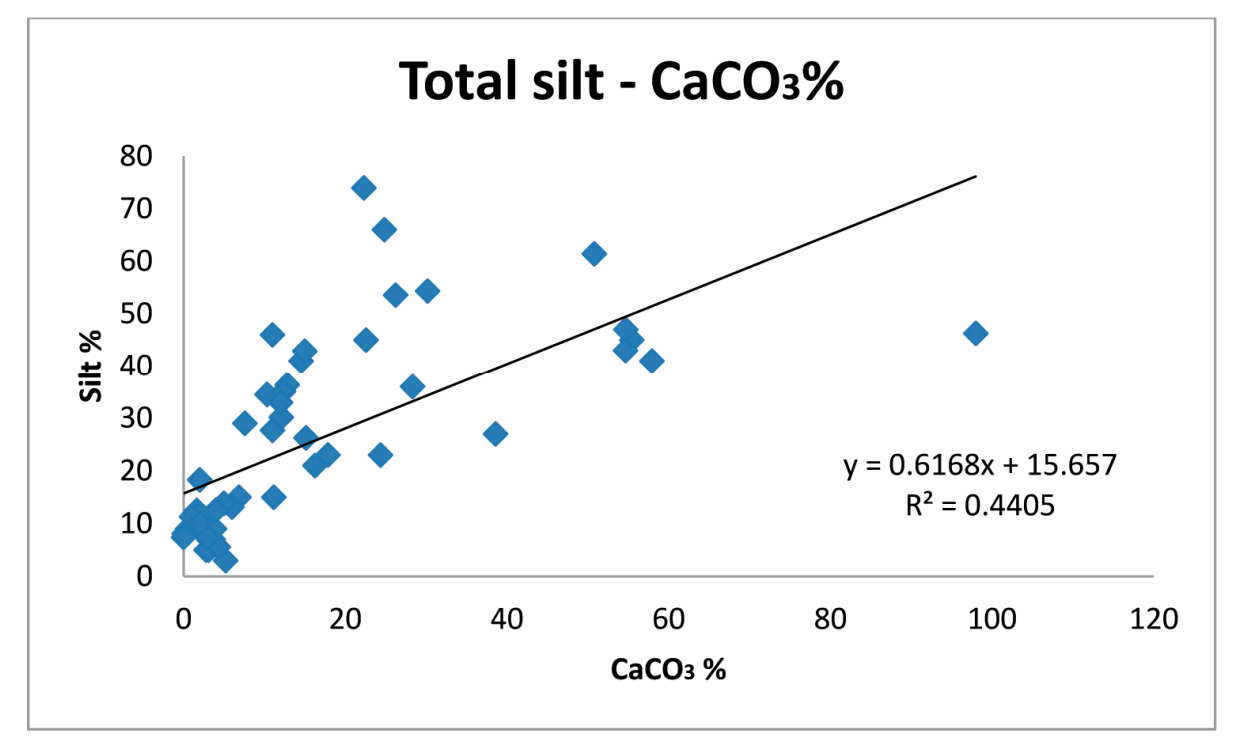

Figure 5. Correlation of silt contents with $\mathrm{CaCO}_{3}$ in the Petra region (referring to the full sample set presented in Table A6 in Appendix C). 
Table 6. $\mathrm{CaCO}_{3}$-contents, grain sizes, and statistical parameters of selected samples from the Petra region. All results are displayed in Table A6 in Appendix C. Dust samples were measured by laser grain size analysis with assumed clay-silt border of $5 \mu \mathrm{m}$ for optimal comparability with Sedigraph results [11,19]. Note that the laser medium silt fraction is mathematically eliminated due to the adapted clay border.

\begin{tabular}{|c|c|c|c|c|c|c|c|c|c|c|c|c|c|c|c|c|c|c|c|}
\hline Sample Name & $\begin{array}{l}\text { Sampl } \\
\text { No. }\end{array}$ & $\begin{array}{c}\mathrm{CaCO}_{3} \\
\%\end{array}$ & $\begin{array}{l}\text { Skeleton > } \\
2 \mathrm{~mm}(\%)\end{array}$ & $\begin{array}{l}\text { Coarse } \\
\text { Sand \% }\end{array}$ & $\begin{array}{l}\text { Medium } \\
\text { Sand \% }\end{array}$ & $\begin{array}{c}\text { Fine } \\
\text { Sand \% }\end{array}$ & $\begin{array}{l}\text { Coarse } \\
\text { Silt } \%\end{array}$ & $\begin{array}{l}\text { Medium } \\
\text { Silt } \%\end{array}$ & $\begin{array}{l}\text { Fine Silt } \\
\quad \%\end{array}$ & $\begin{array}{l}\text { Coarse } \\
\text { Clay \% }\end{array}$ & $\begin{array}{l}\text { Medium } \\
\text { Clay \% }\end{array}$ & $\begin{array}{c}\text { Fine } \\
\text { Clay } \%\end{array}$ & $\begin{array}{c}\text { Sand } \\
\%\end{array}$ & $\begin{array}{l}\text { Silt } \\
\%\end{array}$ & Clay $\%$ & $\begin{array}{c}\text { MODE } 1 \\
{[\mu \mathrm{m}]}\end{array}$ & $\begin{array}{c}\text { MODE } 2 \\
{[\mu \mathrm{m}]}\end{array}$ & $\begin{array}{c}\text { MODE } 3 \\
{[\mu \mathrm{m}]}\end{array}$ & $\begin{array}{c}\text { MEAN } \\
{[\mu \mathrm{m}]}\end{array}$ \\
\hline \multicolumn{20}{|c|}{ Natural Hilltop Soil on Sandstone Plateau: Protic Arenosol (Ochric) } \\
\hline Sandplateau 1 & 47 & 2 & 0 & 3 & 59 & 18 & 6 & 4 & 3 & 3 & 2 & 2 & 80 & 13 & 8 & 415 & 0 & 0 & 143 \\
\hline $\begin{array}{l}\text { Sandplateau Stein } \\
\text { (bedrock) }\end{array}$ & 39 & 0 & 0 & 1 & 75 & 12 & 2 & 2 & 4 & 3 & 1 & 0 & 89 & 7 & 4 & 415 & 0 & 0 & 248 \\
\hline \multicolumn{20}{|c|}{ Jabal Farasha Triclinium Hilltop Ruin: Calcaric Leptosol (Protic) } \\
\hline JF site $124 / 115 \mathrm{~cm}$ & 68 & 10 & 5 & 3 & 16 & 34 & 19 & 9 & 7 & 6 & 3 & 3 & 53 & 34 & 12 & 132 & 0 & 0 & 41 \\
\hline $\begin{array}{l}\text { JF site 124/1 } \\
\text { bedrock }\end{array}$ & 38 & 2 & n.a. & 48 & 10 & 2 & 3 & 8 & 8 & 10 & 9 & 3 & 60 & 18 & 22 & 1315 & 1 & 13 & 89 \\
\hline \multicolumn{20}{|c|}{ Umm Saysaban Hilltop Ruin: Calcaric Leptosol (Protic) } \\
\hline $\begin{array}{c}\text { Umm Saysaban } 10 \\
\mathrm{~cm}\end{array}$ & 71 & 12 & 12 & 5 & 18 & 32 & 13 & 9 & 8 & 8 & 5 & 3 & 54 & 30 & 16 & 132 & 0 & 0 & 36 \\
\hline \multicolumn{20}{|c|}{ Buried Early Holocene Paleosols: Cambic Calcisol (Hypocalcic) (below Shkarat Msaied Ruins); Cambisol (Protocalcic) (below Abu Suwwan Ruins) } \\
\hline Shakarat Msaid 1 & 59 & 15 & 0 & 0 & 30 & 21 & 11 & 7 & 8 & 8 & 6 & 8 & 52 & 26 & 22 & 415 & 4 & 0 & 27 \\
\hline $\begin{array}{l}\text { Abu Suwwan } \\
\text { below nw } 65\end{array}$ & 58 & 18 & 2 & 1 & 39 & 22 & 7 & 8 & 8 & 7 & 5 & 3 & 62 & 23 & 15 & 415 & 4 & 0 & 48 \\
\hline \multicolumn{20}{|c|}{ Monastery Garden of Jabal Haroun: Protic Arenosol (Alcalic. Ochric) } \\
\hline Jh Site $210 \mathrm{~cm}$ & 51 & 4 & 2 & 2 & 63 & 19 & 3 & 2 & 2 & 2 & 3 & 4 & 83 & 7 & 9 & 415 & 0 & 0 & 201 \\
\hline Jh Site $240 \mathrm{~cm}$ & 54 & 3 & 13 & 0 & 50 & 25 & 3 & 2 & 3 & 4 & 6 & 7 & 75 & 9 & 17 & 415 & 0 & 0 & 51 \\
\hline Jh Site $270 \mathrm{~cm}$ & 57 & 4 & 22 & 2 & 52 & 20 & 5 & 2 & 2 & 4 & 5 & 8 & 74 & 9 & 17 & 415 & 0 & 0 & 53 \\
\hline \multicolumn{20}{|c|}{ Terrace on Dolomitic Limestone (Jh site 60): Protic Colluvic Calcaric Regosol } \\
\hline Jh limestone 40 & 74 & 58 & 30 & 6 & 7 & 21 & 14 & 14 & 13 & 12 & 8 & 5 & 34 & 41 & 25 & 132 & 13 & 0 & 13 \\
\hline \multicolumn{20}{|c|}{ Terrace on Sandstone (Jh site 33): Protic Calcaric Arenosol (Colluvic) over Protic Colluvic Calcaric Regosol } \\
\hline Jh site 3310 & 6 & 39 & 26 & 11 & 20 & 35 & 13 & 8 & 7 & 4 & 1 & 1 & $66^{\circ}$ & 27 & 7 & 132 & 0 & 0 & 79 \\
\hline Jh site 3350 & 62 & 7 & 3 & 3 & 21 & 50 & 7 & 4 & 4 & 4 & 3 & 4 & 74 & 15 & 11 & 132 & 0 & 0 & 66 \\
\hline Jh site 3375 & 63 & 15 & 1 & 0 & 2 & 30 & 17 & 12 & 11 & 9 & 8 & 11 & 32 & 41 & 27 & 132 & 0 & 0 & 10 \\
\hline Jh site 33100 & 64 & 23 & 0 & 0 & 3 & 13 & 10 & 16 & 19 & 13 & 6 & 20 & 16 & 45 & 39 & 4 & 132 & 0 & 3 \\
\hline Jh site 33150 & 66 & 24 & 8 & 5 & 19 & 35 & 6 & 7 & 10 & 8 & 5 & 5 & 59 & 23 & 18 & 132 & 4 & 0 & 35 \\
\hline \multicolumn{20}{|c|}{ Reference Samples from Petra Region: Rocks \& Current Fans } \\
\hline $\begin{array}{l}\text { Jh limestone } \\
\text { outcrop }\end{array}$ & 44 & 98 & n.a. & 29 & 14 & 5 & 12 & 27 & 7 & 2 & 1 & 2 & 48 & 46 & 6 & 1315 & 13 & 0 & 74 \\
\hline Beidha Fan & 46 & 16 & 9 & 3 & 43 & 22 & 9 & 5 & 6 & 7 & 3 & 1 & 69 & 21 & 11 & 415 & 1 & 0 & 72 \\
\hline $\begin{array}{l}\text { Fan Umm } \\
\text { Sayhoun }\end{array}$ & 45 & 5 & 7 & 16 & 66 & 9 & 0 & 1 & 1 & 2 & 1 & 3 & 91 & 3 & 6 & 415 & 0 & 0 & 338 \\
\hline Disi Sandstone & 42 & 4 & n.a. & 11 & 67 & 12 & 1 & 2 & 2 & 2 & 1 & 1 & 91 & 6 & 4 & 415 & 0 & 0 & 281 \\
\hline \multicolumn{20}{|c|}{ Current Dust from Sampler on Summit of Jabal Haroun } \\
\hline JH-07-01-17 & 77 & 6 & 0 & 10 & 62 & 11 & 7 & n.a. & 6 & 4 & 0 & 0 & 83 & 13 & 4 & 415 & 0 & 0 & 204 \\
\hline $\begin{array}{l}\mathrm{JH}-15-02-17 \\
\text { (snow) }\end{array}$ & 84 & 15 & 0 & 1 & 24 & 19 & 22 & n.a. & 21 & 13 & 0 & 0 & 44 & 43 & 13 & 415 & 42 & 10 & 34 \\
\hline JH-01-03-17 (rain) & 83 & 11 & 0 & 0 & 11 & 35 & 30 & n.a. & 16 & 8 & 0 & 0 & 46 & 46 & 8 & 132 & 0 & 0 & 40 \\
\hline \multicolumn{20}{|c|}{ Current Dust from Sampler at Foot of of Jabal Haroun } \\
\hline Saleh 05-08-17 & 82 & 11 & 0 & 1 & 23 & 42 & 19 & n.a. & 8 & 6 & 0 & 0 & 65 & 28 & 7 & 132 & 1 & 0 & 85 \\
\hline
\end{tabular}




\subsubsection{Northern Negev}

Table 7 presents grain sizes of selected samples in the Northern Negev. All results are displayed in Table A7 in Appendix C. Silt is the dominating size fraction throughout the sampled profiles. Sand fraction, where present, is mainly fine sand. Small but similar contents of coarse and medium sand are present in nearly all archaeological soils (2-5\% coarse sand, $2-11 \%$ medium sand), with the exception of the upper part of the cistern cleanout spoil. Medium and coarse sand are absent from current dust samples. This could be connected with the position of the sampler ca. $80 \mathrm{~cm}$ above ground.

The main mode of all dust samples is $42 \mu \mathrm{m}$, which is mirrored by most archaeological soils. However, some of them show a main mode of fine sand around $132 \mu \mathrm{m}$.

Clay fraction of current dust storms is between $12 \%$ and $20 \%$, whereas in archaeological soils it is $12-33 \%$. Higher clay contents (29-33\%) were detected in the buried paleosols below ruins, and in the cistern cleanout spoil. Clay fraction of assumed cultivated terrace sediments is between $13 \%$ and $26 \%$, slightly higher, but not as high as in the Petra region.

\subsubsection{Comparison of Grain Sizes of the Archaeological Soils}

A principal component analysis (PCA) of the grain size parameters was performed in order to assess the possible role of different ruin types on grain size distributions. The result is presented in Figure 6, and the whole dataset displayed in Tables A6 and A7 at Appendix C.

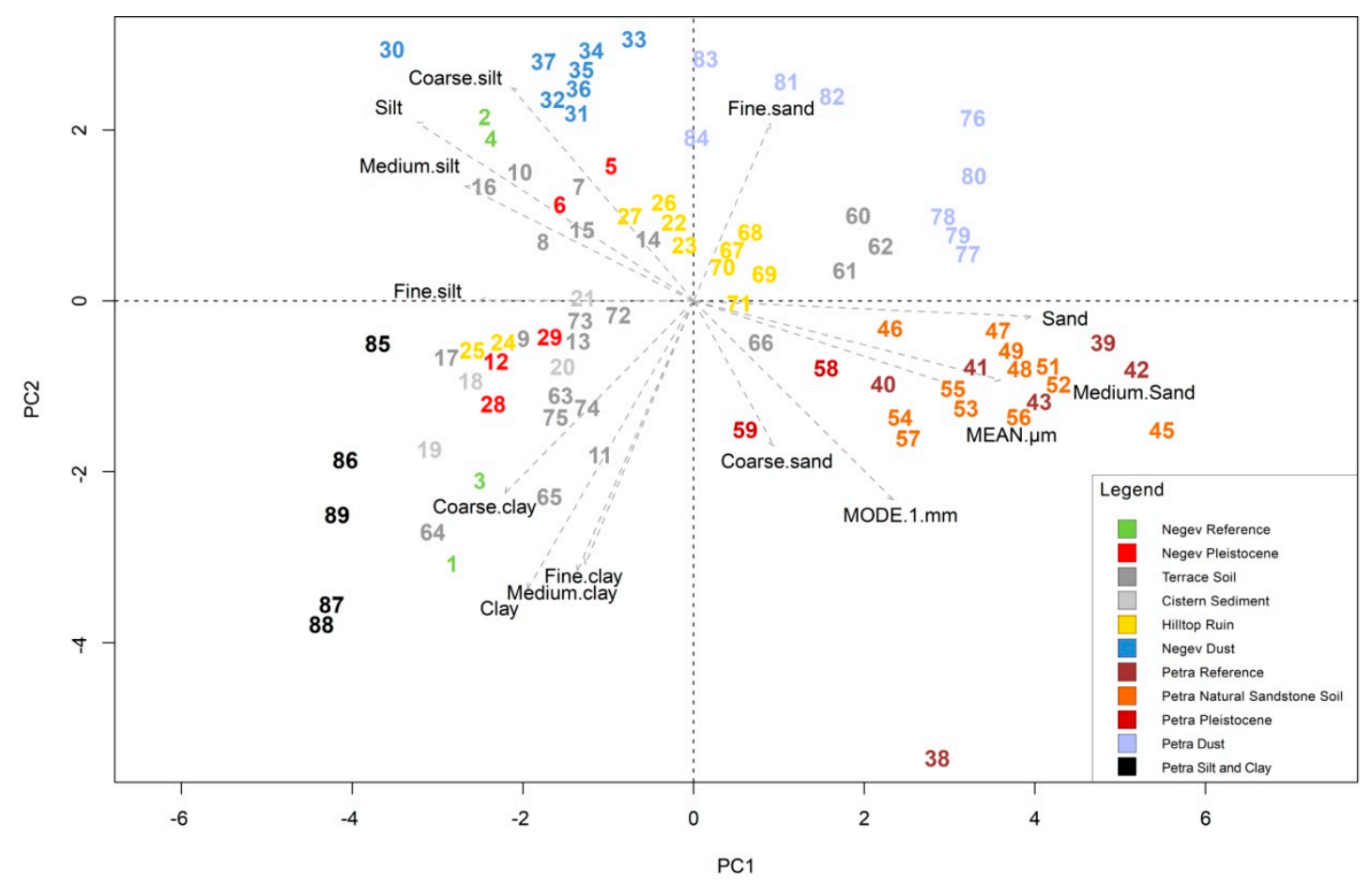

Figure 6. PCA biplot based on the nine grain size classes, and statistical parameters related to grain size distributions, such as the main modes and mean grain sizes of the full dataset (see Tables A6 and A7 in Appendix C). Samples are represented by their numbers and type colors (see Table A8 in Appendix C). The relative locations of the samples reflect their respective similarity with regard to the first and second principal component. The parameters that are most relevant for this distribution are shown at dashed lines, referring to their relative spatial direction with regard to the principal components. 
Table 7. $\mathrm{CaCO}_{3}$-contents, grain sizes, and some statistical parameters of selected samples from the Northern Negev. All results are presented in Table A7 in Appendix C. Dust samples were measured by laser grain size analysis with assumed clay-silt border of $5 \mu \mathrm{m}$ for optimal comparability with Sedigraph results [11,19]. Note that laser medium silt fraction is mathematically eliminated due to the adapted clay border.

\begin{tabular}{|c|c|c|c|c|c|c|c|c|c|c|c|c|c|c|c|c|c|c|c|}
\hline Sample Name & $\begin{array}{c}\text { Samp } \\
\text { No. }\end{array}$ & $\begin{array}{c}\mathrm{CaCO}_{3} \\
\% \\
\end{array}$ & $\begin{array}{l}\text { Skeleton > } \\
2 \mathrm{~mm}(\%)\end{array}$ & $\begin{array}{l}\text { Coarse } \\
\text { Sand \% }\end{array}$ & $\begin{array}{c}\text { Medium } \\
\text { Sand } \%\end{array}$ & $\begin{array}{c}\text { Fine } \\
\text { Sand \% }\end{array}$ & $\begin{array}{l}\text { Coarse } \\
\text { Silt } \% \\
\end{array}$ & $\begin{array}{l}\text { Medium } \\
\text { Silt } \%\end{array}$ & $\begin{array}{c}\text { Fine Silt } \\
\%\end{array}$ & $\begin{array}{l}\text { Coarse } \\
\text { Clay \% } \\
\end{array}$ & $\begin{array}{l}\text { Medium } \\
\text { Clay }\end{array}$ & $\begin{array}{c}\text { Fine } \\
\text { Clay } \%\end{array}$ & $\begin{array}{c}\text { Sand } \\
\%\end{array}$ & $\begin{array}{c}\text { Silt } \\
\%\end{array}$ & $\begin{array}{c}\text { Clay } \\
\%\end{array}$ & $\begin{array}{c}\text { MODE } 1 \\
{[\mu \mathrm{m}]}\end{array}$ & $\begin{array}{c}\text { MODE } 2 \\
{[\mu \mathrm{m}]}\end{array}$ & $\begin{array}{c}\text { MODE } 3 \\
{[\mu \mathrm{m}]}\end{array}$ & $\begin{array}{c}\text { MEAN } \\
{[\mu \mathrm{m}]}\end{array}$ \\
\hline \multicolumn{20}{|c|}{ Terrace on Chalk in Nahal HaRo'a: Protic Colluvic Calcaric Regosol (Siltic) over Calcaric Fluvisol (Aric, Siltic) over Cambic Calcisol (Siltic) } \\
\hline Haroa Farm $10 \mathrm{C}$ & 7 & 24 & 3 & 3 & 2 & 22 & 34 & 15 & 12 & & 3 & 3 & & 61 & 12 & 42 & 0 & 0 & 21 \\
\hline Haroa Farm $28 \mathrm{fAh}$ & 8 & 25 & 12 & 3 & 2 & 20 & 33 & 14 & 12 & 9 & 5 & 2 & 25 & 59 & 16 & 42 & 0 & 0 & 18 \\
\hline Haroa Farm $50 \mathrm{C}$ & 10 & 26 & 13 & 2 & 1 & 16 & 28 & 25 & 14 & 9 & 3 & 1 & 19 & 68 & 13 & 42 & 0 & 0 & 15 \\
\hline Haroa Farm $65 \mathrm{C}$ & 11 & 42 & 57 & 16 & 5 & 16 & 23 & 9 & 9 & 8 & 5 & 9 & 37 & 41 & 22 & 42 & 1315 & 0 & 26 \\
\hline Haroa Farm $80 \mathrm{~B}$ & 12 & 29 & 3 & 1 & 2 & 15 & 30 & 11 & 14 & 11 & 5 & 10 & 18 & 55 & 27 & 42 & 4 & 0 & 10 \\
\hline \multicolumn{20}{|c|}{ Paleosol in Loessial Apron of tributary of Nahal HaRo'a: Cambic Calcisol (Siltic) } \\
\hline $\mathrm{NH}-\mathrm{LA}-30 \mathrm{~cm}$ & 6 & 27 & 5 & 2 & 2 & 23 & 35 & 12 & 10 & 8 & 6 & 1 & 27 & 58 & 15 & 42 & 0 & 0 & 20 \\
\hline \multicolumn{20}{|c|}{ Cistern Cleanout Spoil: Protic Calcaric Regosol (Siltic, Transportic) over Calcaric Regosol (Siltic, Transportic) over Camic Calcisol (Siltic) } \\
\hline HH-WW-Cistern1-Cleanout1-80 & 19 & 34 & 0 & 0 & 1 & 12 & 22 & 16 & 16 & 12 & 8 & 13 & 13 & 54 & 33 & 42 & 4 & 0 & 5 \\
\hline HH-WW-Cistern1-Cleanout1-160 & 21 & 38 & 4 & 3 & 5 & 25 & 25 & 12 & 10 & 8 & 6 & 6 & 33 & 48 & 20 & 42 & 0 & 0 & 16 \\
\hline \multicolumn{20}{|c|}{ Hilltop Ruin Overlooking the Western Wadi: Calcaric Leptosol (Protic, Siltic) } \\
\hline HH-WW-R1 20 & 23 & 35 & 16 & 5 & 11 & 30 & 12 & 16 & 13 & 8 & 3 & 2 & 45 & 42 & 13 & 132 & 13 & 0 & 28 \\
\hline \multicolumn{20}{|c|}{ Hilltop Ruin Overlooking the Central Wadi: Protic Calcaric Regosol (Silttic) over Cambic Calcisol (Siltic) } \\
\hline HH-CW-Ruin 10 & 24 & 40 & 4 & 2 & 3 & 16 & 22 & 14 & 16 & 14 & 6 & 5 & 22 & 53 & 26 & 42 & 4 & 0 & 10 \\
\hline HH-CW-Ruin 50 & 26 & 40 & 9 & 3 & 6 & 32 & 20 & 14 & 13 & 7 & 3 & 2 & 41 & 47 & 12 & 132 & 0 & 0 & 26 \\
\hline HH-CW-Ruin 90 & 29 & 27 & 10 & 2 & 2 & 23 & 22 & 14 & 14 & 11 & 7 & 5 & 27 & 50 & 22 & 132 & 4 & 0 & 13 \\
\hline \multicolumn{20}{|c|}{ Western Wadi Terrace 3: Protic Colluvic Calcaric Regosol (Siltic) } \\
\hline HH-WW-T3-20 & 13 & 36 & 5 & 3 & 4 & 26 & 16 & 14 & 15 & 11 & 6 & 5 & 33 & 44 & 22 & 132 & 4 & 0 & 14 \\
\hline HH-WW-T3-70 & 15 & 48 & 2 & 3 & 4 & 24 & 26 & 16 & 13 & 7 & 4 & 4 & 30 & 55 & 15 & 42 & 0 & 0 & 19 \\
\hline HH-WW-T3-90 & 16 & 43 & 1 & 0 & 1 & 17 & 30 & 21 & 16 & 7 & 3 & 3 & 19 & 68 & 14 & 42 & 0 & 0 & 15 \\
\hline HH-WW-T3-120 & 17 & 41 & 0 & 0 & 1 & 13 & 26 & 19 & 16 & 10 & 7 & 9 & 14 & 61 & 26 & 42 & 0 & 0 & 7 \\
\hline \multicolumn{20}{|c|}{ Negev Reference Samples } \\
\hline Haroa Farm - chalk outcrop & 4 & 79 & n.a. & 0 & 1 & 11 & 1 & 7 & $78^{\circ}$ & 1 & 0 & 2 & 12 & 86 & 2 & 4 & 0 & 0 & 5 \\
\hline HH-WW-C2-soft limestone & 2 & 93 & n.a. & 4 & 3 & 8 & 7 & 53 & 20 & 2 & 1 & 1 & 16 & 80 & 4 & 13 & 132 & 0 & 14 \\
\hline HH-CW-chalk & 3 & 98 & n.a. & 5 & 9 & 11 & 1 & 0 & 38 & 35 & 0 & 2 & 25 & 39 & 36 & 4 & 132 & 0 & 8 \\
\hline \multicolumn{20}{|c|}{ Current Dust from Midreshet Ben Gurion, Sede Boker } \\
\hline Dust storm 24./25.03.03 (rain) & 30 & 35 & 0 & 0 & 0 & 2 & 39 & n.a. & 44 & 14 & 0 & 0 & 2 & 84 & 14 & 42 & 0 & 0 & 11 \\
\hline Dust storm 11.12.10 & 31 & 38 & 0 & 0 & 1 & 23 & 38 & n.a. & 18 & 19 & 1 & 0 & 24 & 56 & 20 & 42 & 1 & 0 & 16 \\
\hline Dust storm 29.02.12 & 33 & 34 & 0 & 0 & 0 & 30 & 46 & n.a. & 11 & 12 & 0 & 0 & 30 & 58 & 12 & 42 & 1 & 0 & 27 \\
\hline Dust storm 22.03.13 & 36 & 44 & 0 & 0 & 1 & 23 & 44 & n.a. & 16 & 16 & 0 & 0 & 24 & 60 & 16 & 42 & 1 & 0 & 17 \\
\hline Dust storm 10/11.02.15 & 37 & 40 & 0 & 0 & 0 & 22 & 44 & n.a. & 15 & 17 & 1 & 0 & 22 & 60 & 18 & 42 & 1 & 0 & 16 \\
\hline
\end{tabular}


Several groups of samples can be discerned. The first group, clustering in the upper right corner, largely represents the dust samples from the Petra region (in light blue), which were collected with dry deposition. They are similar to the sandy layer of the terrace on sandstone, and the most relevant parameter for their position in the diagram is fine sand. Dust samples of wet deposition in the Petra region have an intermediate position between this and the second group.

The second group of samples, clustering in the upper left corner, consists mainly of the dust samples from the Northern Negev (in dark blue), as well as some chalk and soft limestone rocks. The most relevant parameters for their location in the diagram are the silt fractions.

The third group, clustering in the lower right corner, consists of the sandstone samples, the natural soil on the sandstone plateau, and the soil in the monastery garden. The most important parameters for its position in the diagram are sand fractions except fine sand, mean grain size, and main mode.

The fourth group is vaguely identified in the center. It consists of all archaeological soils from both study regions except the monastery garden. Elevated clay contents lead to displacement of some samples to the lower left corner of the diagram, but only the cistern cleanout samples seem to form a group in this direction.

Principle Component PC 1 very well represents fine silt (direction cosine -0.99999) and medium sand (0.96886), whereas PC2 strongly positively correlates with fine sand (direction cosine 0.91814$)$. In summary, the statistical analysis of grain size distributions of the archaeological soils of both study regions indicates they are similar, and distinct from current dust storms and rock outcrops.

The grain sizes were further analyzed statistically using EMMAgeo in order to identify possible end-members of the substrates. The result is displayed in Figure 7 and Table 8. The full list of samples used in this analysis is presented in Appendix C, Table A8.

Table 8. Summary of end members that were statistically modeled with EMMAgeo. The main modes are displayed in bold. Reference samples include rock outcrops and alluvial fans.

\begin{tabular}{|c|c|c|c|c|}
\hline No. & $\begin{array}{c}\text { Explained } \\
\text { Variance \% }\end{array}$ & Composition & Present in & Possible Processes \\
\hline 1 & 8 & $\begin{array}{l}\text { Clay, fine-medium } \\
\text { sand }\end{array}$ & $\begin{array}{c}\text { Soils in both regions, reference } \\
\text { samples, absent from dust } \\
\text { samples }\end{array}$ & $\begin{array}{l}\text { In-situ weathering, local } \\
\text { redistribution }\end{array}$ \\
\hline 2 & 9 & Fine silt, coarse silt & $\begin{array}{l}\text { Most Negev samples; } \\
\text { archaeological soils and dust with } \\
\text { precipitation at Petra }\end{array}$ & Long-range dust transport \\
\hline 3 & 25 & Medium-coarse silt & $\begin{array}{l}\text { All Negev samples; archaeological } \\
\text { soils and dust storms with } \\
\text { precipitation near Petra }\end{array}$ & $\begin{array}{l}\text { Intermediate dust } \\
\text { transport }\end{array}$ \\
\hline 4 & 14 & Fine sand, fine silt & $\begin{array}{l}\text { Negev soils and dust storms; } \\
\text { Petra soils and dust storms }\end{array}$ & $\begin{array}{l}\text { Intermediate dust } \\
\text { transport }\end{array}$ \\
\hline 5 & 35 & Medium sand & $\begin{array}{l}\text { Natural sandstone soils, alluvial } \\
\text { fans, rock outcrops, and dry dust } \\
\text { storms near Petra }\end{array}$ & $\begin{array}{c}\text { Short-range transport from } \\
\text { sandstones }\end{array}$ \\
\hline 6 & 9 & $\begin{array}{l}\text { Medium-coarse sand, } \\
\text { medium silt }\end{array}$ & $\begin{array}{l}\text { Rock outcrops, some soils, alluvial } \\
\text { fans, and dry dust storms near } \\
\text { Petra; paleosols and some } \\
\text { archaeological soils in the Negev }\end{array}$ & $\begin{array}{l}\text { Rock weathering, fluvial } \\
\text { processes, and strong wind } \\
\text { (e.g., dust devils) }\end{array}$ \\
\hline
\end{tabular}

This modeling with EMMAgeo repeated the analysis of [19] with a larger sample set, including additional archaeological soils from different ruin types. Results suggest some statistical smoothing, reducing the number of end members from seven to six (end-member 3 of Lucke et al. [19], which was modeled for a few samples to consist of medium-coarse silt and medium sand, disappeared).

Results from end-member modeling suggest that $\sim 48 \%$ of grain size variance of both investigation regions can be explained by the silt fractions and fine sand, which are probably connected with aeolian transport. Medium sand is found to be a contribution bound to the presence of sandstone rocks of the Petra region. No role of the different types of archaeological ruins is discernible. 

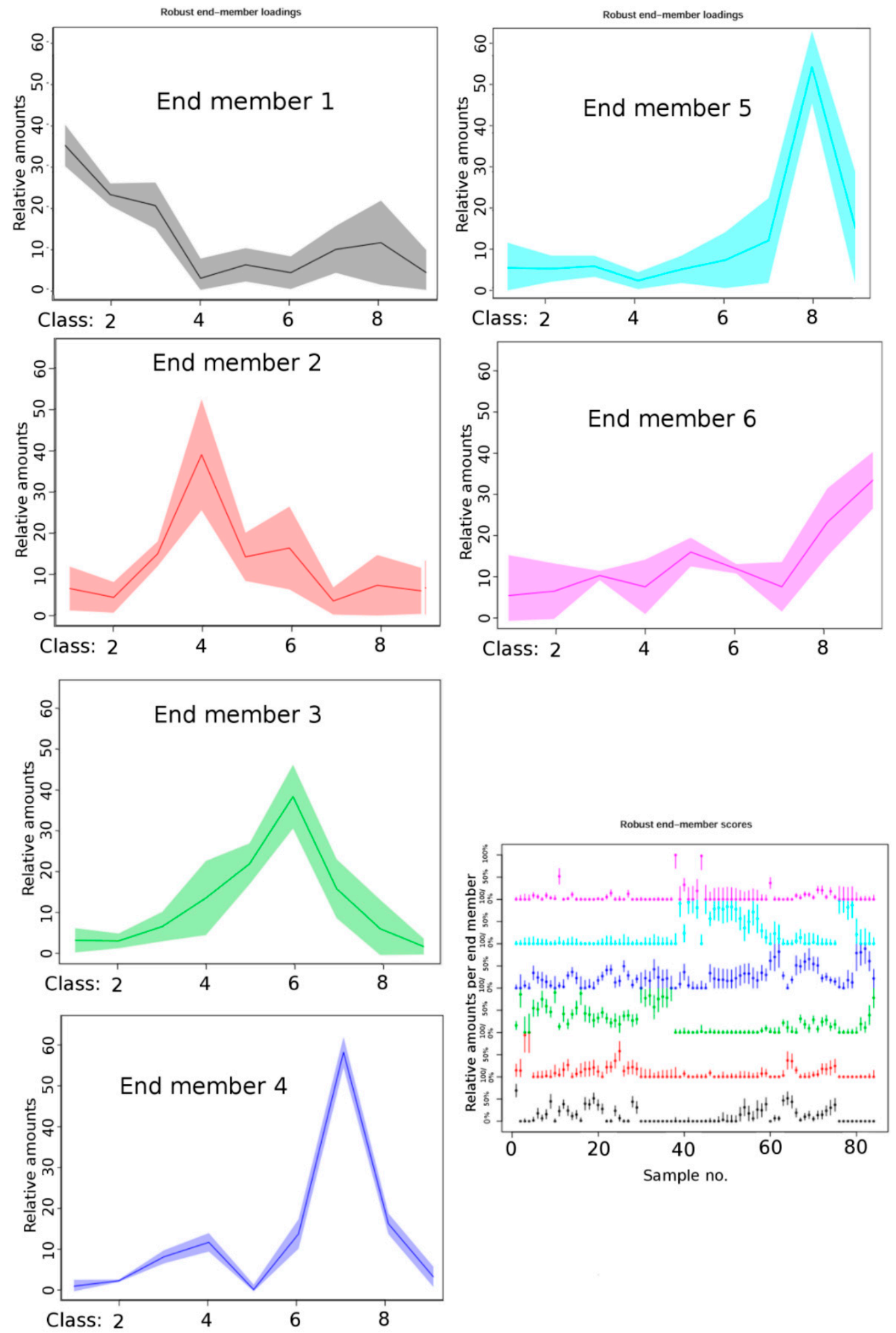

Figure 7. Grain size distributions of six end-members modeled with EMMAgeo, and chart showing the respective end-member scores (relative amounts from $0-100 \%$ ) for each individual sample. Grain-size classes range from 1 (fine clay) to 9 (coarse sand). The chart to the bottom right, showing the robust end-member scores for each sample, displays end-member 1 at the bottom and the subsequent end-members upwards, until end-member 6 at the top. For the detailed list of the samples that were used in calculation of the statistics, including respective individual sample numbers, see Table A8 in Appendix C. 


\subsection{Major and Trace Element Concentrations}

\subsubsection{Petra Region}

Table 9 displays major and trace element concentrations of selected samples from the Petra region. All results are presented in Table A9 at Appendix C. The most relevant element concentrations were plotted against $\mathrm{Al}_{2} \mathrm{O}_{3}$ in Figures 8 and 9.

\section{Major elements}

Strong positive correlations with $\mathrm{Al}_{2} \mathrm{O}_{3}\left(\mathrm{R}^{2}>0.6\right)$ are observed for $\mathrm{TiO}_{2}\left(\mathrm{R}^{2}\right.$ 0.91) and $\mathrm{Fe}_{2} \mathrm{O}_{3}\left(\mathrm{R}^{2}\right.$ 0.78), and negative correlations with $\mathrm{SiO}_{2}\left(\mathrm{R}^{2} 0.63\right)$ are observed (Figure 8). The positive correlation reflects the association of iron and titanium-iron minerals with clays, and the negative trend reflects the antithetic relation to quartz. Weak positive correlations $\left(\mathrm{R}^{2} 0.3-0.6\right)$ are also observed for $\mathrm{K}_{2} \mathrm{O}, \mathrm{CaO}$ and $\mathrm{MgO}$, indicating association with feldspars $\left(\mathrm{K}_{2} \mathrm{O}, \mathrm{CaO}\right)$ and heavy minerals $(\mathrm{MgO})$.

For most oxides, the distribution of the archaeological soils coincides with current dust samples at low $\mathrm{Al}_{2} \mathrm{O}_{3}$ concentrations (Figure 8). The array slope of dust samples, however, is different from that of the archaeological soils, especially for $\mathrm{TiO}_{2}$ and $\mathrm{P}_{2} \mathrm{O}_{5} \cdot \mathrm{SiO}_{2}$ is somewhat exceptional as dust samples and archaeological soils lie approximately on the same negative array, reflecting the contribution of local dust, enriched by quartz, to soils.

A gradual downward decrease of oxides, except for $\mathrm{Al}_{2} \mathrm{O}_{3}$ and $\mathrm{SiO}_{2}$, is observed at the natural soil profile on sandstone (Sandplateau samples). This trend and the low concentrations of oxides indicate an upward increase in contribution of dust to the weathered sandstone (Table 9).

Exceptional enrichment by $\mathrm{CaO}$ and $\mathrm{MgO}$ is observed at the top sample of the terrace on sandstone $(\mathrm{JH}$ site $3310 \mathrm{~cm})$. The only possible source for the enrichment is the adjacent dolomitic limestone slope. At the profile on dolomitic limestone (Jh site 60) some oxides $\left(\mathrm{Fe}_{2} \mathrm{O}_{4}, \mathrm{TiO}_{2}, \mathrm{Na}_{2} \mathrm{O}\right.$, and $\left.\mathrm{K}_{2} \mathrm{O}\right)$ coincide with the dust field at low $\mathrm{Al}_{2} \mathrm{O}_{3}$ range, whereas $\mathrm{SiO}_{2}$, contributed by adjacent sandstone, and $\mathrm{CaO}$ and $\mathrm{MgO}$, contributed by bedrock, lie away from both dust and soils. $\mathrm{P}_{2} \mathrm{O}_{5}$ is in-between dust array and archeological soils trendline, indicating a unique source for phosphorous (possibly partly from manuring and dung, see [60]).

Oxide values of the sandstone bedrocks and natural soils mostly lie near or at the field where archaeological soils low in $\mathrm{Al}_{2} \mathrm{O}_{3}$ and current dust coincide, but are apparently not related to either array. The archaeological soils are enriched, compared to rocks and dust, by all oxides, except $\mathrm{SiO}_{2}$. Elevated $\mathrm{MgO}$ - and $\mathrm{CaO}$-concentrations are observed at the terrace soil on dolomitic limestone, suggesting a steady contribution of the surrounding exposures to all size fractions. Hence, weathering products of the dolomitic limestone played a role in the composition of its associated soils. Moreover, it seems likely that the dolomitic limestone outcrop contributed to the adjacent topsoil of the sandstone terrace (sample JH site $3310 \mathrm{~cm}$ ).

The data considered and presented above (see Table 9, Figure 8) indicate that both rock outcrops and dust contribute to the archeological soils in the Petra region, but not alone. $\mathrm{Al}_{2} \mathrm{O}_{3}$ is much higher in many archeological soil samples than in dust and sandstones, and strong correlation with most oxides is observed for that high $\mathrm{Al}_{2} \mathrm{O}_{3}$ range. It is suggested that this is due to preferential fixation of elements by silt and clay fractions, probably connected with surface crusts [19]. Another possibility is that clayey beds within the sandstone sequence serve as a third local source. These beds, the result of Cambrian tropical weathering, are dominated by fine sand containing significant amounts of kaolinite [58] that is accompanied by enriched concentrations of many oxides and trace elements (see below). As the clayey beds are easily erodible, their chemical impact could be more effective than their mass.

\section{$\underline{\text { Trace elements }}$}

$\mathrm{Ti} / \mathrm{Zr}$-ratios of the archaeological soils lie in the range of 4-11, except a sample of the sandstone terrace with a ratio of 15 (Table 9). In current dust, high ratios are reached only in the sample deposited 
with rain on 1 March 2017. Apart from that, current dust has Ti/Zr-ratios of 4-7, which are similar to most sandstones, fans, and natural soils on sandstone.

$\mathrm{Cr}, \mathrm{Ga}, \mathrm{Nb}$, Th, V, Y, Zn have strong $\left(\mathrm{R}^{2}>0.6\right)$ positive correlations with $\mathrm{Al}_{2} \mathrm{O}_{3}$ (Figure 9) in the archaeological soils, whereas $\mathrm{Pb}, \mathrm{Rb}$, and $\mathrm{Sr}$ have weak positive correlations ( $\mathrm{R}^{2}$ 0.3-0.6). Distribution of most trace elements coincides with current dust at low contents of $\mathrm{Al}_{2} \mathrm{O}_{3}$, as for major oxides. Slopes of trendlines are, however, mostly different from those of the diffused array of current dust. Ba and $\mathrm{Rb}$ are exceptional as dust samples and archaeological soils lie approximately on the same trendline, probably due to association with feldspars and not with clays. Outlier $\mathrm{Zn}$ concentrations are observed in some current dust samples, probably due to air pollution.

Trace element distribution of sandstones and natural soils on sandstone mostly lie near or at the field of archaeological soils and dust at a low $\mathrm{Al}_{2} \mathrm{O}_{3}$ range. Most trace elements are not related to the trendlines of archaeological soils (especially $\mathrm{Nb}, \mathrm{Sr}$, and $\mathrm{Zr}$ ), but some seem to be similar to the arrays of current dust: $\mathrm{Ga}, \mathrm{Nb}, \mathrm{V}$, and $\mathrm{Y}$.

The archaeological soils are enriched by various trace elements including $\mathrm{Ba}, \mathrm{Cr}, \mathrm{Ga}, \mathrm{Ni}, \mathrm{Rb}, \mathrm{Sr}$, Th, $\mathrm{V}$, and $\mathrm{Y}$, relative to rocks and dust, except for the dust deposited with rain, which is similarly enriched. These enrichments are associated with high concentrations of $\mathrm{TiO}_{2}, \mathrm{Al}_{2} \mathrm{O}_{3}$, and $\mathrm{Fe}_{2} \mathrm{O}_{3}$. This supports the suggestions presented above for major oxides.

Trace elements of the terrace on dolomitic limestone (Jh site 60) have three characteristics: (1) they coincide with current dust array (regarding $\mathrm{Ba}, \mathrm{Cr}, \mathrm{Ga}, \mathrm{Rb}$, and $\mathrm{Sr}$ ); (2) they coincide with the trendline of archaeological soils on sandstone (regarding $\mathrm{Ba}, \mathrm{Ga}, \mathrm{Nb}, \mathrm{Rb}, \mathrm{Zn}$ ); and (3) they are unrelated to any other group (regarding Th, V). Exceptional is their $\mathrm{Zr}$ distribution, which is associated with the sandstone group and may indicate a common source for the Zr-bearing minerals for the latter and the insoluble residue in the dolomitic limestone. 
Table 9. Element concentrations and Ti/Zr-ratio of selected samples from the Petra region. LOI is loss on ignition. All results are displayed in Table A9 at Appendix C. The sample no. reflects the sample's number used in statistical analyses, ordered according to types of deposits (see Table A8 in the Appendix C).

\begin{tabular}{|c|c|c|c|c|c|c|c|c|c|c|c|c|c|c|c|c|c|c|c|c|c|c|c|c|c|c|c|}
\hline Sample Name & $\begin{array}{c}\text { Sample } \\
\text { No. }\end{array}$ & $\begin{array}{c}\mathrm{SiO}_{2} \\
\%\end{array}$ & $\begin{array}{c}\mathrm{TiO}_{2} \\
\%\end{array}$ & $\underset{\%}{\mathrm{Al}_{2} \mathrm{O}_{3}}$ & $\underset{\%}{\mathrm{Fe}_{2} \mathrm{O}_{3}}$ & $\underset{\%}{\mathrm{MnO}}$ & $\underset{\%}{\mathrm{MgO}}$ & $\begin{array}{c}\mathrm{CaO} \\
\%\end{array}$ & $\underset{\%}{\mathrm{Na}_{2} \mathrm{O}}$ & $\begin{array}{c}\mathrm{K}_{2} \mathrm{O} \\
\%\end{array}$ & $\underset{\%}{\mathrm{P}_{2} \mathrm{O}_{5}}$ & $\begin{array}{c}\text { LOI } \\
\%\end{array}$ & $\underset{\%}{\text { Sum }}$ & $\begin{array}{c}\mathrm{Ba} \\
\mathrm{ppm}\end{array}$ & $\begin{array}{c}\mathrm{Cr} \\
\mathrm{ppm}\end{array}$ & $\begin{array}{r}\mathrm{Ga} \\
\mathrm{ppm}\end{array}$ & $\begin{array}{c}\mathrm{Nb} \\
\mathrm{ppm}\end{array}$ & $\begin{array}{c}\mathrm{Ni} \\
\mathrm{ppm}\end{array}$ & $\begin{array}{l}(\mathrm{Pb}) \\
\mathrm{ppm}\end{array}$ & $\begin{array}{c}\mathbf{R b} \\
\text { ppm }\end{array}$ & $\begin{array}{c}\text { Sr } \\
\text { ppm }\end{array}$ & $\begin{array}{c}\text { Th } \\
\text { ppm }\end{array}$ & $\begin{array}{c}\mathrm{V} \\
\mathrm{ppm}\end{array}$ & $\begin{array}{c}\mathrm{Y} \\
\mathrm{ppm}\end{array}$ & $\begin{array}{c}\mathrm{Zn} \\
\mathrm{ppm}\end{array}$ & $\begin{array}{c}\mathrm{Zr} \\
\mathrm{ppm}\end{array}$ & $\mathrm{Ti} / \mathrm{Zr}$ \\
\hline \multicolumn{28}{|c|}{ Natural Hilltop Soil on Sandstone Plateau: Protic Arenosol (Ochric) } \\
\hline Sandplateau 1 & 47 & 89.3 & 0.28 & 3.4 & 0.86 & 0.017 & 0.39 & 1.3 & 0.05 & 0.25 & 0.06 & 3.3 & 99.1 & 86 & 17 & 5.0 & 5.0 & 4.0 & 14 & 7.0 & 104 & 5.0 & 23 & 15 & 21 & 193 & 9 \\
\hline Sandplateau St & 39 & 92.9 & 0.09 & 3.7 & 0.10 & 0.003 & 0.02 & 0.1 & 0.01 & 0.03 & 0.07 & 1.6 & 98.6 & 23 & 6 & 5.0 & 3.0 & 1.0 & 11 & 1.0 & 83 & 10.0 & 12 & 12 & 4 & 75 & 7 \\
\hline \multicolumn{28}{|c|}{ Jabal Farasha Triclinium Hilltop Ruin: Calcaric Leptosol (Protic) } \\
\hline JF site $124 / 15 \mathrm{~cm}$ & 67 & 69.1 & 0.60 & 7.2 & 2.46 & 0.036 & 1.26 & 7.5 & 0.54 & 0.77 & 0.22 & 10.1 & 99.8 & 249 & 50 & 7.8 & 13.0 & 15.9 & 17 & 24.3 & 271 & 9.2 & 51 & 24 & 40 & 410 & 9 \\
\hline JF site $124 / 15 \mathrm{~cm} \mathrm{S \& C}$ & 85 & 48.4 & 0.92 & 11.4 & 4.37 & 0.066 & 2.12 & 12.5 & 0.74 & 1.19 & 1.24 & 16.8 & 99.7 & 376 & 76 & 11.3 & 28.3 & 39.3 & 22 & 38.0 & 450 & 11.1 & 84 & 33 & 71 & 541 & 10 \\
\hline JF site $124 / 1$ bedrock & 38 & 54.1 & 1.00 & 21.5 & 11.84 & 0.007 & 0.13 & 1.5 & 0.24 & 0.40 & 0.29 & 8.8 & 99.7 & 243 & 113 & 27.4 & 17.0 & 27.5 & 107 & 14.0 & 786 & 18.2 & $\begin{array}{l}04 \\
92\end{array}$ & 58 & 13 & 182 & 33 \\
\hline \multicolumn{28}{|c|}{ Umm Saysaban Hilltop Ruin: Calcaric Leptosol (Protic) } \\
\hline Umm Saysaban $10 \mathrm{~cm}$ & 71 & 69.5 & 0.61 & 8.2 & 2.11 & 0.028 & 0.96 & 7.5 & 0.58 & 0.50 & 0.22 & 9.6 & 99.8 & 183 & 41 & 9.2 & 13.8 & 12.5 & 24 & 17.3 & 325 & 9.8 & 47 & 24 & 35 & 422 & 9 \\
\hline $\begin{array}{l}\text { Umm Saysaban } 10 \mathrm{~cm} \\
\text { S\&c }\end{array}$ & 86 & 44.9 & 0.88 & 13.9 & 3.25 & 0.052 & 1.96 & 13.9 & 0.59 & 0.94 & 1.13 & 18.2 & 99.8 & 304 & 75 & 16.3 & 26.8 & 24.5 & 23 & 32.1 & 493 & 13.3 & 73 & 34 & 65 & 503 & 11 \\
\hline \multicolumn{28}{|c|}{ Buried Early Holocene Paleosols: Cambic Calcisol (Hypocalcic) (below Shkarat Msaied Ruins) } \\
\hline Shakarat Msaid 1 & 59 & 72.1 & 0.45 & 4.5 & 2.03 & 0.034 & 1.24 & 8.6 & 0.54 & 0.74 & 0.14 & 9.5 & 99.9 & 138 & 44 & 5.5 & 10.3 & 10.3 & 9 & 19.5 & 159 & 6.9 & 47 & 18 & 29 & 332 & 9 \\
\hline Shakarat Msaid 1 S \& C & 88 & 45.0 & 0.78 & 9.0 & 3.73 & 0.066 & 2.33 & 16.9 & 0.73 & 1.28 & 1.18 & 18.7 & 99.8 & 306 & 81 & 11.1 & 27.2 & 34.0 & 6 & 32.4 & 300 & 8.1 & 73 & 27 & 63 & 405 & 12 \\
\hline \multicolumn{28}{|c|}{ Monastery Garden of Jabal Haroun: Protic Arenosol (Alcalic. Ochric) } \\
\hline Jh Site $210 \mathrm{~cm}$ & 51 & 88.7 & 0.23 & 1.8 & 1.21 & 0.015 & 0.43 & 2.6 & 0.38 & 0.25 & 0.21 & 4.0 & 99.9 & 76 & 22 & 1.0 & 6.1 & 6.6 & 8 & 7.9 & 121 & 5.1 & 26 & 12 & 26 & 263 & 5 \\
\hline Jh Site $240 \mathrm{~cm}$ & 54 & 87.8 & 0.30 & 2.4 & 1.42 & 0.018 & 0.39 & 2.5 & 0.37 & 0.28 & & 4.3 & 99.9 & 92 & 27 & 4.8 & 8.0 & 9.3 & 7 & 9.6 & 146 & 6.6 & 28 & 16 & 26 & 431 & 4 \\
\hline Jh Site $270 \mathrm{~cm}$ & 57 & 84.8 & 0.37 & 3.0 & 2.33 & 0.031 & 0.51 & 3.1 & 0.56 & 0.39 & 0.11 & 4.7 & 99.8 & 150 & 39 & 7.2 & 9.0 & 25.3 & 11 & 12.6 & 182 & 7.4 & 41 & 21 & 33 & 491 & 5 \\
\hline \multicolumn{28}{|c|}{ Terrace on Dolomitic Limestone (Jh site 60): Protic Colluvic Calcaric Regosol } \\
\hline Jh limestone 60 & 75 & 34.0 & 0.40 & 4.4 & 2.14 & 0.032 & 4.97 & 25.2 & 0.39 & 0.53 & 0.19 & 27.6 & 99.9 & 210 & 46 & 2.1 & 8.4 & 6.7 & 5 & 15.5 & 335 & 6.0 & 50 & 14 & 32 & 250 & 10 \\
\hline Jh limestone $60 \mathrm{~S} \& \mathrm{C}$ & 89 & 27.0 & 0.50 & 4.8 & 2.42 & 0.038 & 5.27 & 28.5 & 0.40 & 0.66 & 0.55 & 29.7 & 99.8 & 262 & 54 & 4.2 & 15.4 & 26.7 & 6 & 18.6 & 396 & 6.4 & 51 & 18 & 38 & 279 & 11 \\
\hline \multicolumn{28}{|c|}{ Terrace on Sandstone (Jh site 33): Protic Calcaric Arenosol (Colluvic) over Protic Colluvic Calcaric Regosol } \\
\hline Jh site 33 & 60 & 50.4 & 0.37 & 4.1 & 1.67 & 0.027 & 3.84 & 17.6 & 0.36 & 1.01 & 0.21 & 20.2 & 99.9 & 272 & 36 & 4.7 & 9.5 & 9.1 & 7 & 27.4 & 267 & 5.8 & 38 & 16 & 35 & 349 & 6 \\
\hline & 64 & $\begin{array}{l}30.4 \\
47.8\end{array}$ & 0.70 & $\begin{array}{l}4.1 \\
13.5\end{array}$ & 3.97 & 0.039 & $\begin{array}{l}3.04 \\
2.25\end{array}$ & 12.1 & 0.45 & 2.24 & 0.23 & 16.6 & 99.8 & 440 & 52 & $\begin{array}{l}4.8 \\
15.7\end{array}$ & $\begin{array}{l}9.3 \\
15.3\end{array}$ & $\begin{array}{l}9.1 \\
26.1\end{array}$ & 14 & 79.8 & 356 & $\begin{array}{l}5.0 \\
11.9\end{array}$ & 6 & 23 & $\begin{array}{l}30 \\
82\end{array}$ & 284 & $\begin{array}{l}0 \\
15\end{array}$ \\
\hline Jh site $33100 \mathrm{~S} \& \mathrm{C}$ & 87 & 40.5 & 0.86 & 15.3 & 4.32 & 0.045 & 2.41 & 14.7 & 0.44 & 2.17 & 0.47 & 18.6 & 99.8 & 433 & 54 & 16.7 & 20.8 & 31.1 & 16 & 81.7 & 419 & 14.7 & 56 & 27 & 94 & 295 & 18 \\
\hline \multicolumn{28}{|c|}{ Reference Samples from Petra Region: Rocks \& Current Fans } \\
\hline Jh limestone 60 & 44 & 3.1 & & 0.7 & 0.29 & 0.013 & 18.35 & 31.9 & & 0.02 & 0.07 & 45.5 & 100.0 & 61 & 16 & 2.3 & 1.2 & 0.5 & 1 & & & & & & 7 & & 18 \\
\hline & 46 & 71.3 & 0. & 2.9 & 1.61 & & 1.06 & 11.4 & & & & 9.8 & & 196 & 48 & 8.8 & & & 8 & & & & 40 & 19 & 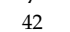 & & 5 \\
\hline Disi Sandstone & 42 & 92.9 & 0.13 & 2.0 & 0.14 & 0.001 & 0.20 & 1.4 & 0.37 & 0.03 & 0.07 & 2.7 & 99.9 & 39 & 13 & $\begin{array}{l}3.0 \\
3.4\end{array}$ & 3.3 & $\begin{array}{l}2.49 \\
7.9\end{array}$ & 5 & 1.7 & 237 & $\begin{array}{l}5.0 \\
5.2\end{array}$ & 21 & 6 & 7 & 88 & 6 \\
\hline \multicolumn{28}{|c|}{ Mountaintop dust Petra region } \\
\hline JH-07-01-17 & 77 & 88.2 & 0.25 & 1.7 & 1.23 & 0.010 & 0.39 & 3.2 & 0.53 & 0.18 & & 3.9 & & 74 & 25 & 7.8 & 6.4 & & 13 & & 190 & 7.2 & 26 & 10 & 47 & 64 & 6 \\
\hline & 84 & & 0. & 3.8 & 2.14 & & 1.16 & 8.5 & & 0.46 & & 10.0 & & 185 & 49 & 5.2 & 10.2 & & 12 & & & & 45 & 19 & 63 & & 7 \\
\hline JH-01-03-17 (rain) & 83 & 73.8 & 0.80 & 3.5 & 1.52 & 0.022 & 0.97 & 6.5 & 0.57 & 0.42 & 0.37 & 11.3 & 99.8 & 190 & 39 & 1.9 & 9.8 & 15.6 & 14 & 14.0 & 244 & 8.5 & 41 & 18 & 269 & 474 & 10 \\
\hline \multirow{2}{*}{\multicolumn{28}{|c|}{ Foot of mountain dust Petra region }} \\
\hline Saleh 25-11-16 & 80 & 93.6 & 0.18 & 0.6 & 0.81 & 0.005 & 0.11 & 1.3 & 0.34 & & & & & & 15 & 1.0 & 4.2 & 9.0 & 6 & 2.5 & 149 & 6.1 & 16 & 7 & 31 & 234 & 5 \\
\hline
\end{tabular}




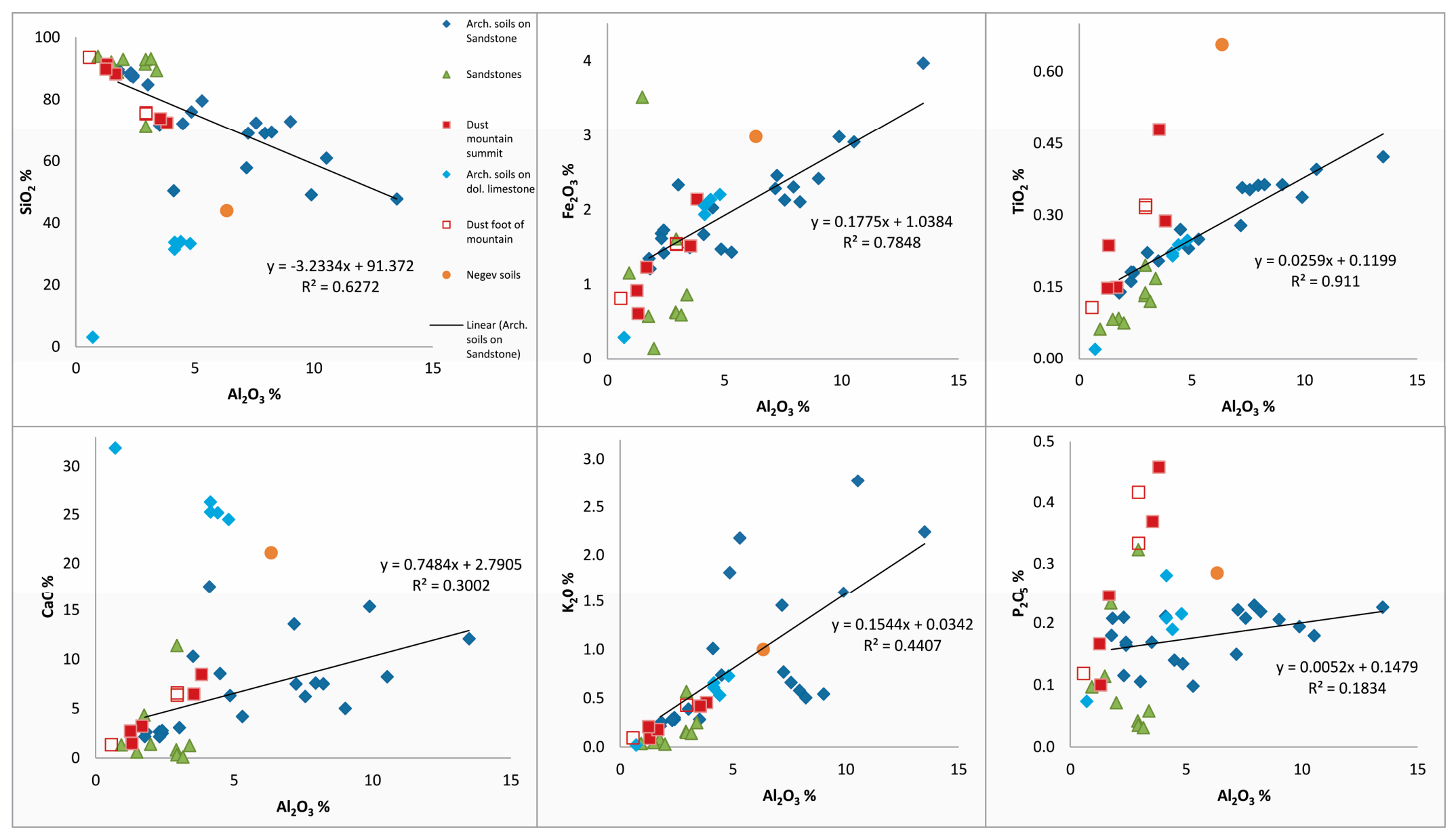

Figure 8. Selected major elements, presented as oxides, plotted against $\mathrm{Al}_{2} \mathrm{O}_{3}$ of samples from the Petra region (see Table A9, except the outlier sample JF 124/1 rock and the silt\&clay fractions samples). For comparison, average of Northern Negev archeological soils is shown with a light brown circle. A linear trendline was calculated for archaeological soils on sandstone alone. 


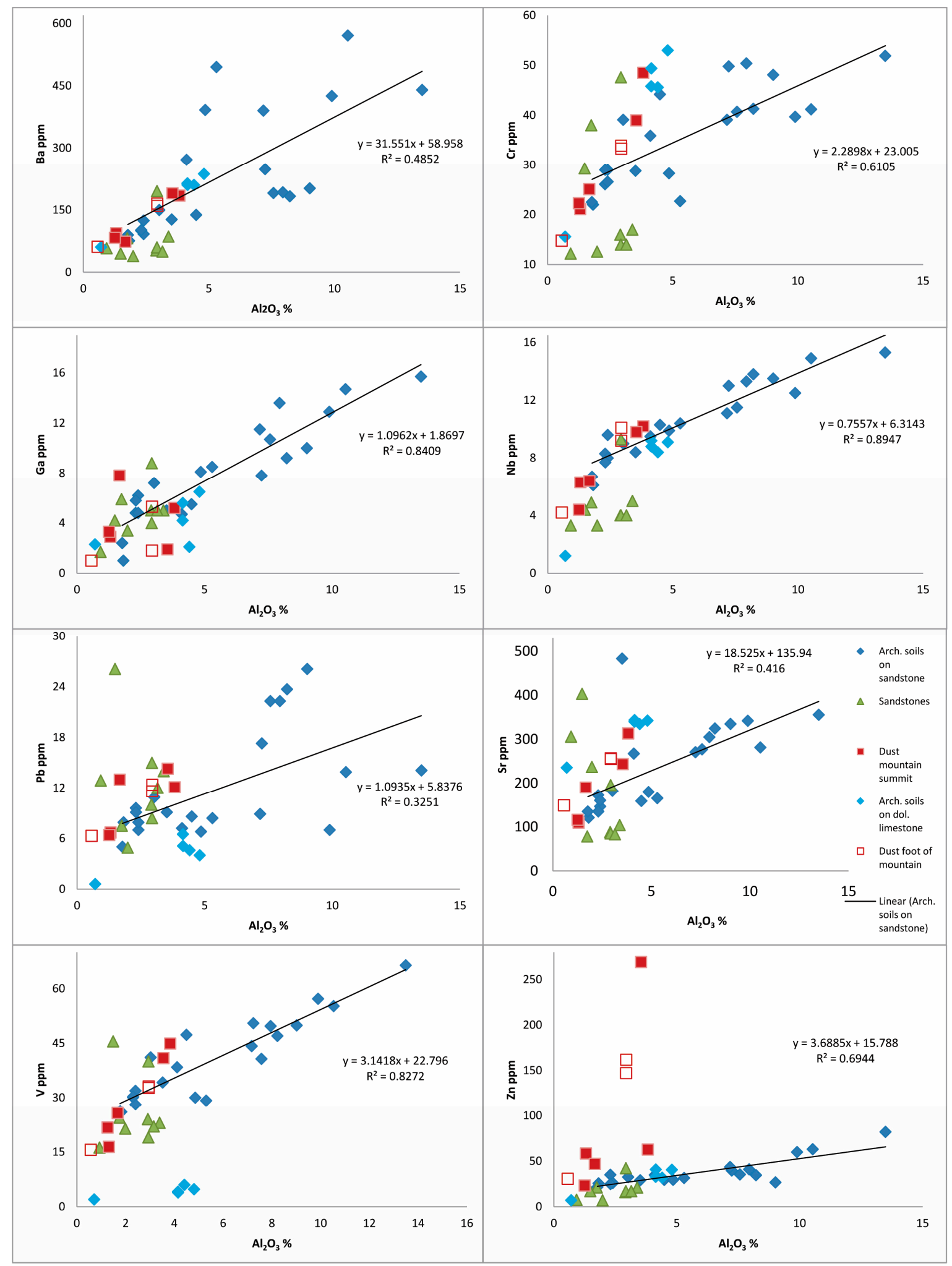

Figure 9. Selected trace elements plotted against $\mathrm{Al}_{2} \mathrm{O}_{3}$ of samples from the Petra region (see Table A9 except the outlier sample JF 124/1 rock and the silt\&clay fractions samples). A linear regression trendline was calculated for archaeological soils on sandstone alone. 


\subsubsection{Northern Negev}

Table 10 displays concentrations of major and trace elements of selected samples from the Northern Negev. All results are presented in Table A10 at Appendix C. Some of the most relevant element concentrations were plotted against $\mathrm{Al}_{2} \mathrm{O}_{3}$ in Figures 10 and 11 .

Major elements

In the archaeological soils and paleosols, a strong positive correlation with $\mathrm{Al}_{2} \mathrm{O}_{3}\left(\mathrm{R}^{2}>0.6\right)$ is observed only for $\mathrm{Fe}_{2} \mathrm{O}_{3}\left(\mathrm{R}^{2}\right.$ 0.92) (Figure 10). Weak positive correlations $\left(\mathrm{R}^{2}\right.$ 0.3-0.6) are discerned for $\mathrm{SiO}_{2}$ and $\mathrm{TiO}_{2}$, whereas a negative trendline is observed for $\mathrm{CaO}\left(\mathrm{R}^{2}\right.$ 0.41). For most oxides, current dust and soils fields coincide, except $\mathrm{K}_{2} \mathrm{O}$, which is lower in some dust samples than in soils. $\mathrm{P}_{2} \mathrm{O}_{5}$ concentrations of the hilltop ruin soil at the central wadi (samples HH-CW-Ruin) are relatively high, possibly representing remains of bones or other kitchen refuse, as was observed in hilltop ruin soils in Northern Jordan [61].

Oxides concentrations of limestone bedrocks are hardly related to the distribution fields of dust and archeological soils. Exceptional is $\mathrm{Fe}_{2} \mathrm{O}_{3}$, which falls on a correlation trendline common for all samples, including bedrocks. This suggests a consistent and similar chemical behavior of $\mathrm{Al}$ and $\mathrm{Fe}$ in oxidized weathering environments of sedimentary rocks, sediments and soils in the region as has been recorded in previous studies [62-64]. In contrast to the Petra region, enrichment of $\mathrm{TiO}_{2}, \mathrm{Al}_{2} \mathrm{O}_{3}, \mathrm{Fe}_{2} \mathrm{O}_{3}$ or of other oxides is not observed in the archaeological soils.

The upper, younger part of the cistern cleanout spoil shows slightly higher contents of $\mathrm{Al}_{2} \mathrm{O}_{3}$, $\mathrm{Fe}_{2} \mathrm{O}_{3}, \mathrm{MgO}$, and $\mathrm{MnO}$ than the lower, older part, while contents of $\mathrm{CaO}$ are slightly smaller. This might correspond to higher clay contents of the younger cistern cleanout, and could indicate a change of dust sources.

The Northern Negev archeological soils and dust are more uniform in the major oxides composition than those from the Petra region, as they lie within the same field/array. However, samples from the terrace in the tributary of Nahal HaRo'a are relatively depleted in $\mathrm{CaO}$, and enriched in $\mathrm{SiO}_{2}$. This seems connected with paleosols like those of the loessial apron as potential contributors to terrace sediments in the tributary of Nahal HaRo'a. Remains of such paleosols were found at various adjacent locations, e.g., below the cistern cleanout pile, suggesting a wider distribution during the late Pleistocene time with remains possibly still preserved during antiquity.

Although the chemical composition of current dust largely resembles that of the archaeological soils, the sample deposited with rain is an outlier. It contains significantly higher concentrations of $\mathrm{Al}_{2} \mathrm{O}_{3}, \mathrm{Fe}_{2} \mathrm{O}_{3}$, and $\mathrm{MgO}$, has an exceptional high Ti/Zr-ratio of 22, and relatively low concentrations of $\mathrm{SiO}_{2}$ and $\mathrm{CaO}$. This indicates some contribution of leached Mediterranean soils of the Terra Rossa type.

\section{$\underline{\text { Trace elements }}$}

The Northern Negev archeological soils and dust show Ti/Zr ratios of 9-12. Rocks, in contrast, show ratios of 12-33. Most analyzed trace elements in archaeological soils ( $\mathrm{Sr}, \mathrm{Ba}, \mathrm{Zr}, \mathrm{Ni}, \mathrm{Pb}, \mathrm{Cr}, \mathrm{Rb}, \mathrm{Th}$, and $\mathrm{Zn}$ ) have very weak $\left(\mathrm{R}^{2}<0.3\right)$ positive correlations with $\mathrm{Al}_{2} \mathrm{O}_{3}$, whereas a few $(\mathrm{Ga}, \mathrm{Nb}, \mathrm{V}$, and $\mathrm{Y})$ have higher correlations $\left(R^{2} 0.5-0.6\right)$ (Figure 11). This may indicate multiple sources for trace elements.

For most trace elements, current dust and soils lie within the same field. $\mathrm{Zn}$ is elevated in current dust, along with $\mathrm{Ba}$, and in the hilltop ruin soil at the central wadi (samples $\mathrm{HH}-\mathrm{CW}$-Ruin), along with $\mathrm{P}_{2} \mathrm{O}_{5}$. Enrichment of zinc in dust is known to be a result of industrial pollution [65]. 
Table 10. Element concentrations and Ti/Zr-ratios of selected samples from the Northern Negev. LOI is loss on ignition. All results are displayed in Table A10 at Appendix C. The sample no. reflects the sample's number used in statistical analyses, ordered according to types of deposits (see Table A8 in the Appendix C).

\begin{tabular}{|c|c|c|c|c|c|c|c|c|c|c|c|c|c|c|c|c|c|c|c|c|c|c|c|c|c|c|c|}
\hline Sample Name & $\begin{array}{l}\text { Sample } \\
\text { No. }\end{array}$ & $\begin{array}{c}\mathrm{SiO}_{2} \\
\%\end{array}$ & $\begin{array}{c}\mathrm{TiO}_{2} \\
\%\end{array}$ & $\underset{\%}{\mathrm{Al}_{2} \mathrm{O}_{3}}$ & $\begin{array}{c}\mathrm{Fe}_{2} \mathrm{O}_{3} \\
\%\end{array}$ & $\underset{\%}{\mathrm{MnO}}$ & $\underset{\%}{\mathrm{MgO}}$ & $\begin{array}{c}\mathrm{CaO} \\
\%\end{array}$ & $\begin{array}{c}\mathrm{Na}_{2} \mathrm{O} \\
\%\end{array}$ & $\begin{array}{c}\mathrm{K}_{2} \mathrm{O} \\
\%\end{array}$ & $\begin{array}{c}\mathrm{P}_{2} \mathrm{O}_{5} \\
\%\end{array}$ & $\begin{array}{c}\text { LOI } \\
\%\end{array}$ & 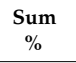 & $\begin{array}{c}\text { Ba } \\
\text { ppm }\end{array}$ & $\begin{array}{c}\mathrm{Cr} \\
\mathrm{ppm}\end{array}$ & $\begin{array}{c}\mathrm{Ga} \\
\mathrm{ppm}\end{array}$ & $\begin{array}{c}\mathrm{Nb} \\
\mathrm{ppm}\end{array}$ & $\begin{array}{c}\mathrm{Ni} \\
\text { ppm }\end{array}$ & $\begin{array}{l}(\mathrm{Pb}) \\
\mathrm{ppm}\end{array}$ & $\begin{array}{c}\mathbf{R b} \\
\mathbf{p p m}\end{array}$ & $\begin{array}{c}\mathrm{Sr} \\
\mathrm{ppm}\end{array}$ & $\begin{array}{c}\text { Th } \\
\text { ppm }\end{array}$ & $\begin{array}{c}\mathrm{V} \\
\mathrm{ppm}\end{array}$ & $\begin{array}{c}\mathrm{Y} \\
\mathrm{ppm}\end{array}$ & $\begin{array}{c}\mathrm{Zn} \\
\mathrm{ppm}\end{array}$ & $\begin{array}{c}\mathrm{Zr} \\
\mathrm{ppm}\end{array}$ & $\mathrm{Ti} / \mathrm{Zr}$ \\
\hline \multicolumn{28}{|c|}{ Terrace on Chalk in Nahal HaRo'a: Protic Colluvic Calcaric Regosol (Siltic) over Calcaric Fluvisol (Aric, Siltic) over Cambic Calcisol (Siltic) } \\
\hline Haroa Farm $10 \mathrm{C}$ & 7 & 49.2 & 0.73 & 6.6 & 3.2 & 0.067 & 2.6 & 14.9 & 1.16 & 1.15 & 0.27 & 19.9 & 99.8 & 357 & 77 & 10.6 & 14 & 18.1 & 5.7 & 30 & 407 & 5.4 & 70 & 21 & 43 & 473 & 9 \\
\hline Haroa Farm $28 \mathrm{fAh}$ & 8 & 50.1 & 0.73 & 6.8 & 3.4 & 0.066 & 2.5 & 16.0 & 1.21 & 0.91 & 0.23 & 17.9 & 99.8 & 331 & 77 & 14.7 & 15 & 24.6 & 7.0 & 23 & 417 & 6.5 & 75 & 23 & 45 & 465 & 9 \\
\hline Haroa Farm $50 \mathrm{C}$ & 10 & 48.7 & 0.75 & 7.3 & 3.6 & 0.067 & 2.6 & 16.8 & 1.22 & 0.95 & 0.21 & 17.6 & 99.8 & 324 & 77 & 8.3 & 14 & & 3.3 & 24 & 486 & & 78 & 22 & 44 & & 11 \\
\hline Haroa Farm 65 & 11 & 38.9 & 0.53 & 5.3 & 2.6 & 0.051 & 2.0 & 25.7 & 0.79 & 0.63 & 0.24 & 23.1 & 99.8 & 262 & 56 & 3.3 & 12 & 16.4 & 5.6 & 16 & 480 & 4.7 & 58 & 19 & 35 & 297 & 11 \\
\hline Haroa Farm 80 B & 12 & 48.4 & 0.95 & 7.5 & 3.9 & 0.075 & 2.4 & 17.6 & 1.21 & 1.06 & 0.23 & 16.4 & 99.8 & 330 & 110 & 9.5 & 17 & 32.2 & 4.5 & 29 & 419 & 6.5 & 94 & 26 & 45 & 536 & 11 \\
\hline \multicolumn{28}{|c|}{ Paleosol in Loessial Apron of tributary of Nahal HaRo'a: Cambic Calcisol (Siltic) } \\
\hline NH-LA-30cm & 6 & 53.8 & 0.89 & 6.6 & 3.3 & 0.064 & 2.3 & 15.9 & 0.96 & 1.23 & 0.18 & 14.6 & 99.8 & 337 & 90 & 11.1 & 16 & 21.1 & 6.4 & 30 & 342 & 8.2 & 76 & 24 & 41 & 657 & 8 \\
\hline \multicolumn{28}{|c|}{ Cistern Cleanout Spoil: Protic Calcaric Regosol (Siltic, Transportic) over Calcaric Regosol (Siltic, Transportic) } \\
\hline HH-WW-C.1-Cl.80 & 19 & 44.0 & 0.67 & 7.7 & 3.7 & 0.063 & 2.2 & 20.3 & 0.83 & 0.82 & 0.23 & 19.4 & 99.8 & 325 & 77 & 8.7 & 13 & 27.1 & 3.8 & 22 & 354 & 5.5 & 75 & 21 & 52 & 337 & 12 \\
\hline HH-WW-C1-Cl-160 & 21 & 44.7 & 0.59 & 6.1 & 2.7 & 0.050 & 1.9 & 22.1 & 0.76 & 0.83 & 0.20 & 19.8 & 99.8 & 300 & 65 & 5.7 & 11 & 21.3 & 6.3 & 24 & 367 & 6.5 & 61 & 20 & 38 & 374 & 10 \\
\hline \multicolumn{28}{|c|}{ Hilltop Ruin Overlooking the Western Wadi: Calcaric Leptosol (Protic, Siltic) } \\
\hline HH-WW-R1 20 & 23 & 39.8 & 0.59 & 5.8 & 2.6 & 0.045 & 2.2 & 23.8 & 0.60 & 0.52 & 0.24 & 23.6 & 99.8 & 282 & 62 & 4.2 & 11 & 7.8 & 4.3 & 13 & 418 & 6.8 & 63 & 17 & 38 & 392 & 9 \\
\hline \multicolumn{28}{|c|}{ Hilltop Ruin Overlooking the Central Wadi: Protic Calcaric Regosol (Siltic) over Cambic Calcisol (Siltic) } \\
\hline HH-CW-Ruin 10 & 24 & 39.8 & 0.62 & 6.3 & 3.0 & 0.061 & 2.8 & 23.0 & 0.67 & 1.23 & 0.60 & 21.7 & 99.8 & 326 & 68 & 6.8 & 13 & 20.5 & 6.5 & 31 & 493 & 4.8 & 69 & 20 & 63 & 372 & 10 \\
\hline HH-CW-Ruin & 26 & 39.5 & 0.57 & 5.5 & 2.5 & 0.053 & 3.1 & 23 & 0.82 & & 0.67 & 22.3 & 99.8 & 327 & 62 & 4.9 & 12 & 19.3 & 6.0 & 25 & & & 55 & 18 & 58 & 68 & 9 \\
\hline HH-CW-Ruin 90 & 29 & 48.9 & 0.75 & 6.1 & 2.5 & 0.050 & 2.3 & 18.1 & 0.95 & 0.94 & 0.20 & 19.0 & 99.8 & 295 & 55 & 4.5 & 12 & 9.0 & 10.3 & 22 & 363 & 6.4 & 80 & 18 & 34 & 477 & 9 \\
\hline \multicolumn{28}{|c|}{ Western Wadi Terrace 3: Protic Colluvic Calcaric Regosol (Siltic) } \\
\hline & 13 & 45.1 & 0.66 & 6.7 & 3.1 & 0.056 & 1.9 & 21.4 & 0.69 & 1.01 & 0.23 & 18.9 & 99.8 & 338 & 65 & 9.8 & 13 & & & & & & & & & & 10 \\
\hline HH-WW-T3-70 & 1 & 43.2 & 0.59 & 5.9 & 2.6 & 0.05 & 2.0 & 23.6 & 0.69 & & 0.29 & 19.9 & 99.8 & & 167 & 5.7 & 12 & 98. & 0.8 & 24 & 358 & 3.9 & 58 & 19 & 39 & 369 & 10 \\
\hline HH-WW-T3-90 & 16 & 40.8 & 0.56 & 5.9 & 2.7 & 0.050 & 2.2 & 23.9 & & 0.88 & 0.23 & & & 300 & 56 & 6.5 & 12 & 16.1 & 3.7 & 23 & & 5.1 & 61 & 18 & 40 & 314 & 11 \\
\hline HH-WW-T3-120 & 17 & 40.6 & 0.57 & 6.7 & 3.1 & 0.048 & 2.4 & 23.1 & 0.64 & 0.91 & 0.22 & 21.7 & 99.8 & 300 & 66 & 5.8 & 13 & 19.8 & 6.9 & 25 & 349 & 6.1 & 63 & 19 & 44 & 291 & 12 \\
\hline \multicolumn{28}{|c|}{ Negev Reference Samples } \\
\hline HH-CW-chalk & 3 & 1.1 & 0.03 & 0.2 & 0.2 & 0.003 & 0.6 & 54.5 & 0.01 & 0.02 & 0.08 & 43.2 & 99.9 & 135 & 15 & 3.1 & 3 & 0.5 & 1.2 & 1 & 266 & 2.9 & 14 & 1 & 5 & 15 & 14 \\
\hline \multicolumn{28}{|c|}{ Current Dust from Midreshet Ben Gurion, Sede Boker } \\
\hline & 30 & & & & 4.5 & 0.069 & 3.7 & 19.9 & 0.86 & 1.09 & 0.30 & 20.6 & 99.8 & 305 & 66 & 10.2 & 16 & 28.8 & 7.1 & 34 & 430 & 8.3 & 87 & 23 & 78 & 169 & 22 \\
\hline 11.12 .10 & 31 & 41.9 & 0.64 & 6.1 & 2.8 & 0.056 & 2.1 & 21.6 & 0.94 & 0.48 & 0.30 & 22.8 & 99.8 & 438 & 76 & 5.9 & 12 & 26.8 & 7.5 & 10 & 521 & 5.8 & 78 & 21 & 77 & 400 & 10 \\
\hline dust storn & 33 & 47. & 0.6 & 5.7 & 2.7 & 0.0 & 2.3 & & & 0. & 0. & & 99 & & 66 & 4.3 & 12 & & 6. & 16 & & 4 & & & 8 & & 8 \\
\hline & 36 & & 0.6 & 5.5 & 2. & & 2 & & & 0. & & & & 4 & 8 & 6.7 & 11 & & 3. & & & & & & 68 & & 9 \\
\hline 11..02.15 & 37 & 38.8 & 0.68 & 6.6 & 3.5 & 0.062 & 3.1 & 22.3 & 0.88 & 0.47 & 0.26 & 23.2 & 99.8 & 486 & 76 & 7.2 & 14 & 25.6 & 6.1 & 11 & 528 & 7.4 & 79 & 27 & 74 & 440 & 9 \\
\hline
\end{tabular}




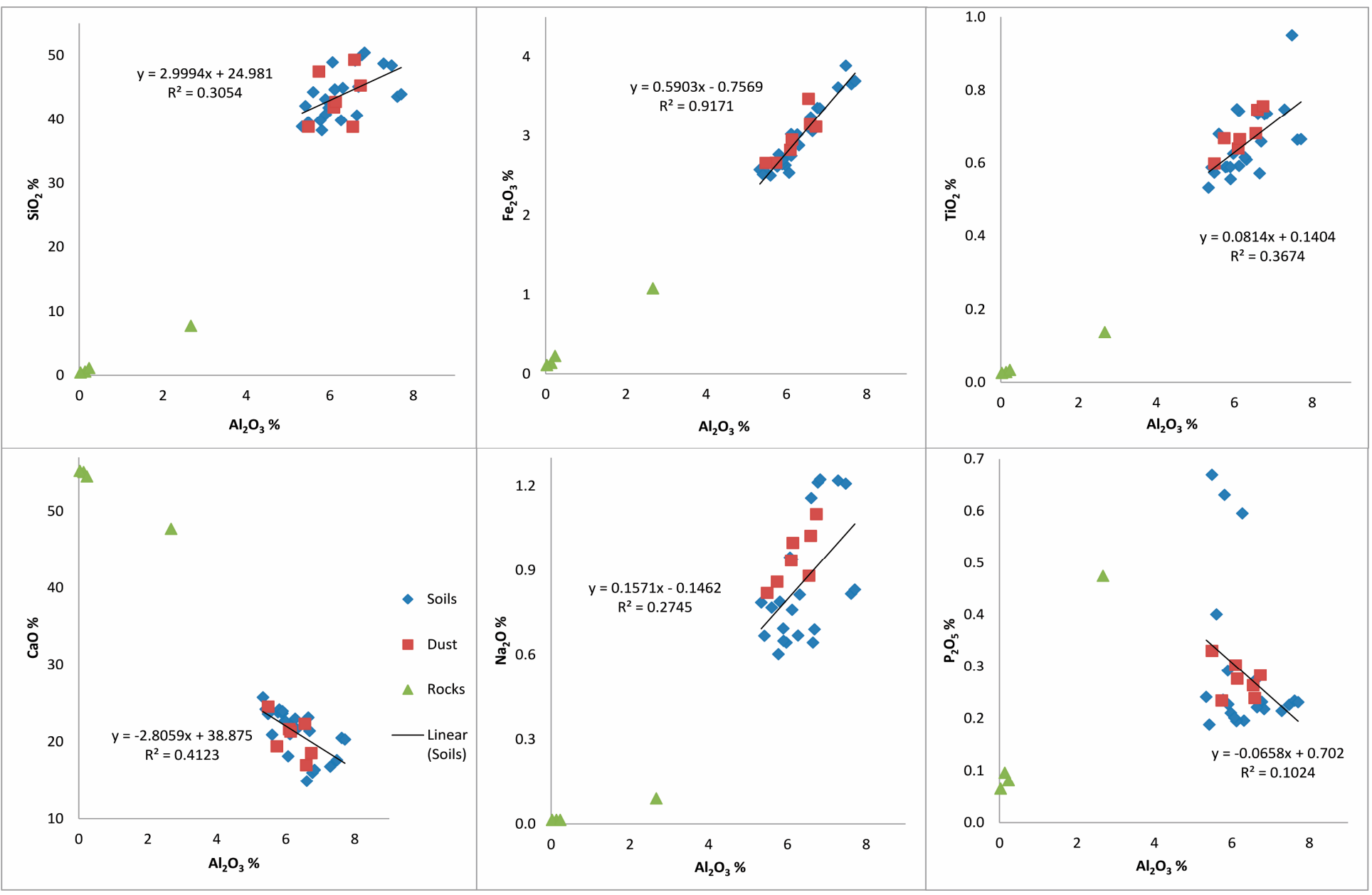

Figure 10. Selected major elements, presented as oxides, are plotted against $\mathrm{Al}_{2} \mathrm{O}_{3}$ of samples from the Northern Negev (see Table A10). A linear regression was calculated and presented for the archeological soils alone. 


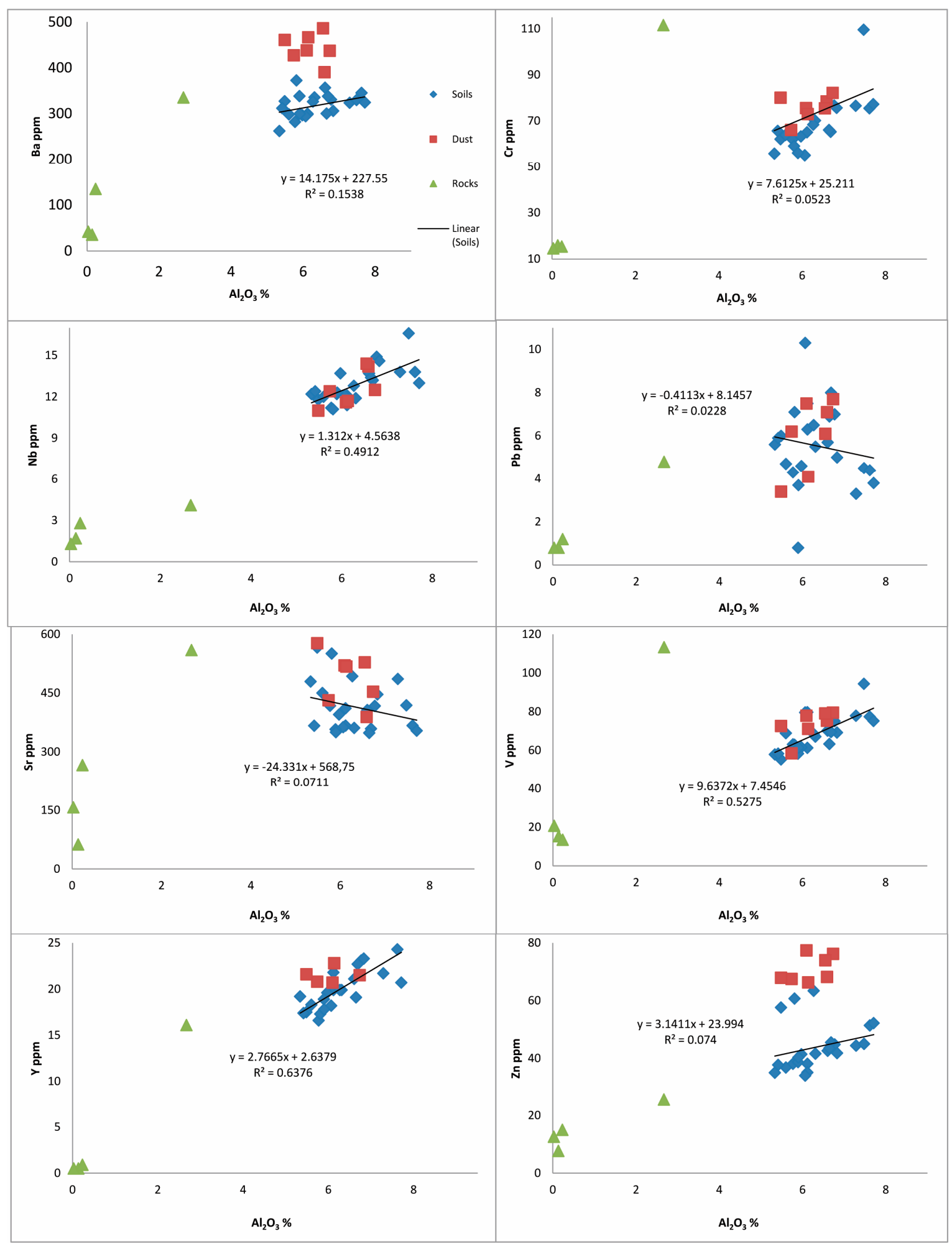

Figure 11. Selected trace elements plotted against $\mathrm{Al}_{2} \mathrm{O}_{3}$ of samples from the Northern Negev (see Table A10, excluding the paleosols of the loessial aprons near Nahal HaRo'a, the Turonian paleosol, and the outlier sample of dust deposited with rain). A linear regression was calculated and presented for the archeological soils alone.

$\mathrm{Rb}$ is somewhat exceptional as some dust samples have lower concentrations than archaeological soils in a similar manner to $\mathrm{K}_{2} \mathrm{O}$. As $\mathrm{Rb}$ is known to be geochemically associated with $\mathrm{K}$, mainly due to 
co-occurrence in K-feldspars and micas, this suggests a common mineralogical origin and also a slight shift of dust provenance with time (Figure 12).
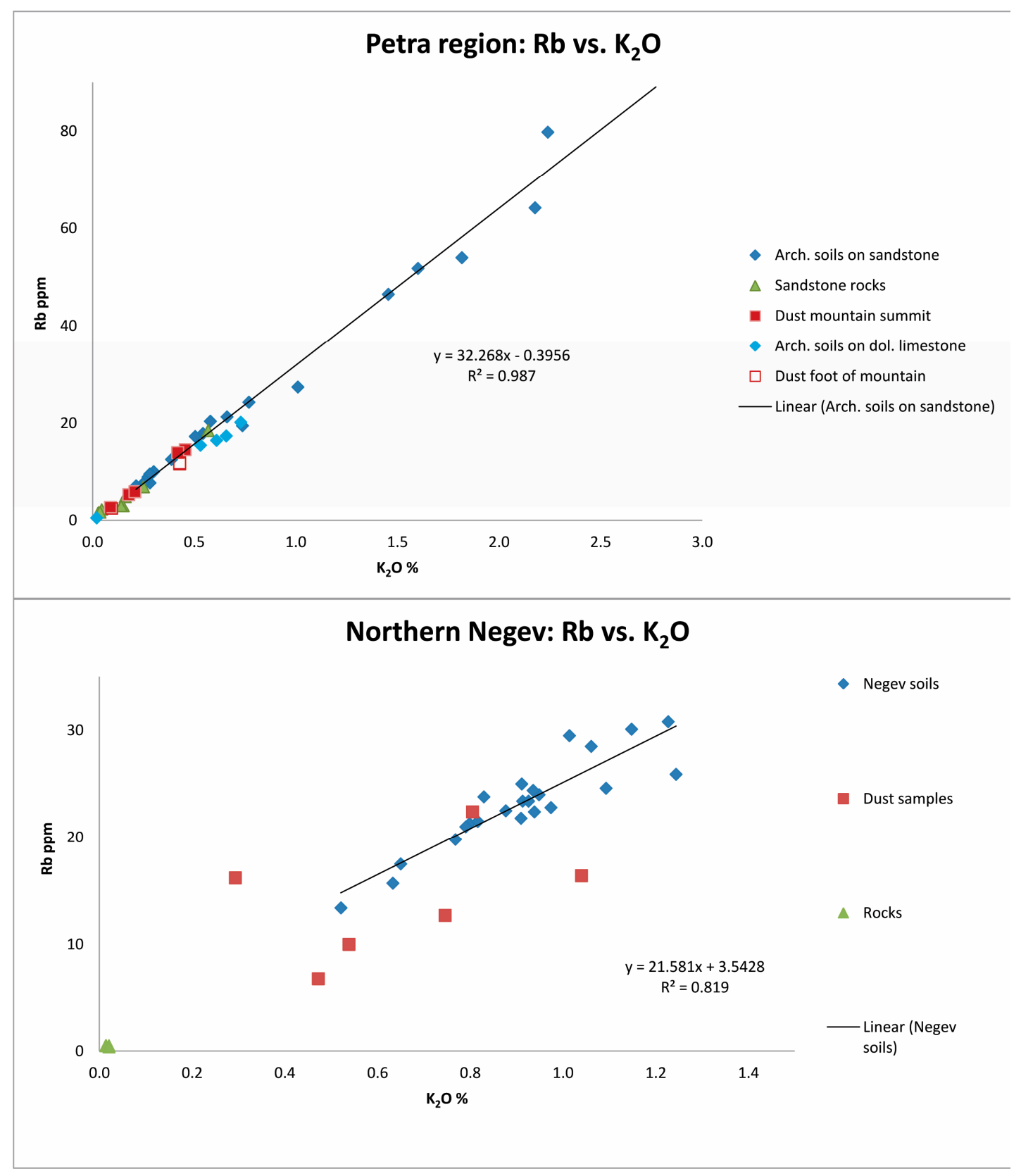

Figure 12. Plots of $\mathrm{Rb}$ against $\mathrm{K}_{2} \mathrm{O}$ concentrations in the Petra region (top) and Northern Negev (bottom). The linear regressions were calculated and presented for archaeological soils on sandstone in the Petra region, and for soils in the Northern Negev.

Trace elements suggest rock outcrops to have a negligible contribution to the archeological soils in the Northern Negev. The tight similarity to dust, however, indicates the latter to be the main parent material. The sample deposited with rain is an outlier as it has relatively low concentrations of $\mathrm{Ba}$ and $\mathrm{Zr}$ and high concentrations of $\mathrm{Ga}, \mathrm{Nb}, \mathrm{Ni}, \mathrm{Rb}, \mathrm{Th}$, and $\mathrm{V}$. 


\subsubsection{Statistical Comparison of Element Concentrations}

A principal component analysis (PCA) of the element concentrations was performed in order to assess the possible role of the different ruin types and investigation areas for their distribution. The result is presented in Figure 13. The respective dataset is found in Tables A8 and A9 at Appendix C.

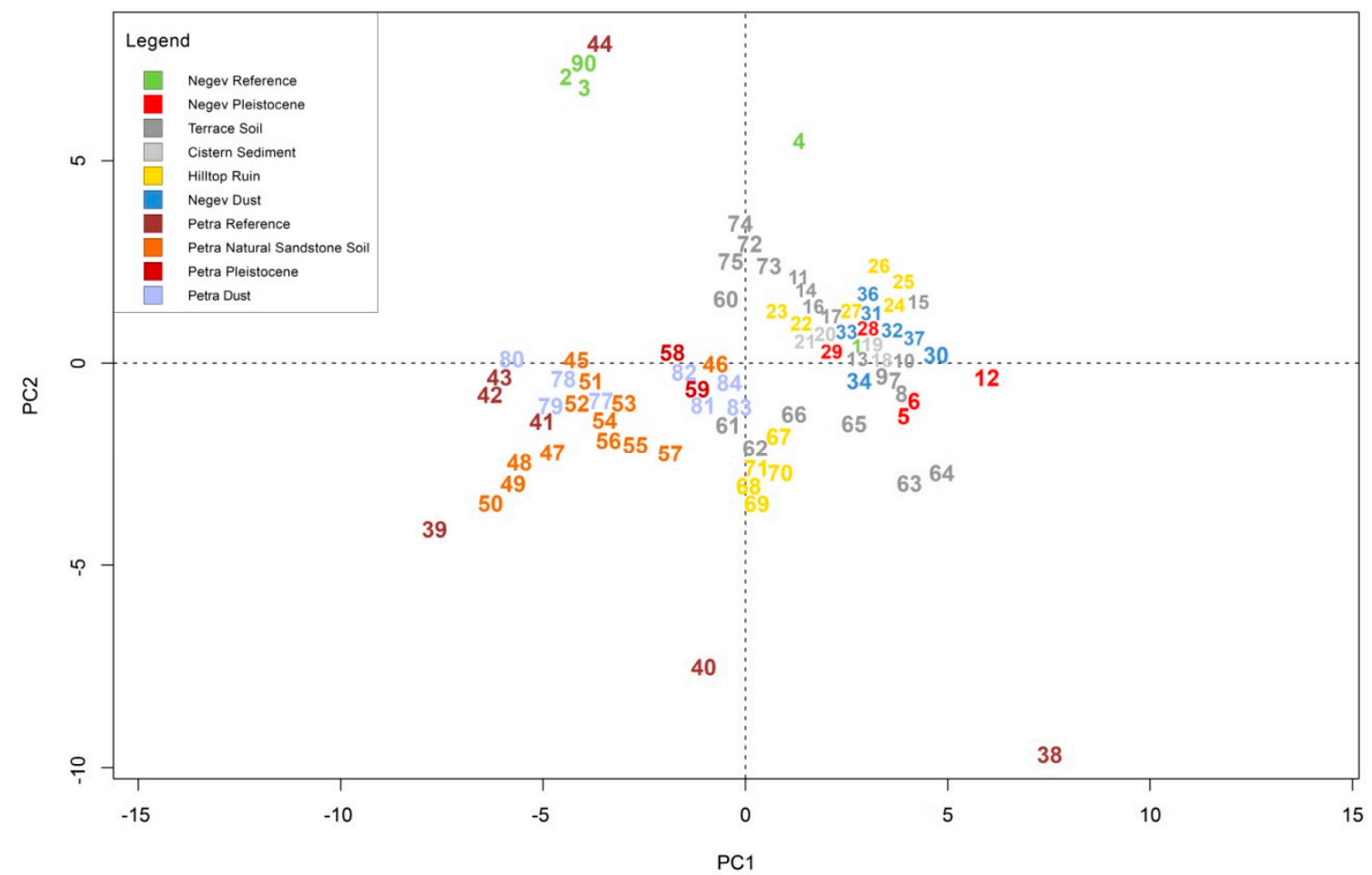

Figure 13. PCA plot based on element concentrations. The locations of samples reflect their relative similarity with regard to the first and second principal component. Samples are represented by their numbers and type colors (see Table A8 in Appendix C).

The PCA plot shows that the geochemical composition of the dust and most Northern Negev archeological soils are so similar that it is difficult to display sample names separately. These samples cluster at the upper-right corner with slightly positive values of both principal components (PCs). Only the samples from the tributary of Nahal HaRo'a are, to some degree, different: the paleosol of the loessial apron (samples NH-LA, no. 5-6), as well as the paleosol below the terrace in the tributary of Nahal HaRo'a (no. 12), show small negative values of PC2. The same is true for the buried Ah-horizon in the tributary of Nahal HaRo'a terrace profile (no. 8).

Rock samples of the Northern Negev are, except one chalk, chemically similar, but distinct from the soil and dust samples. They show negative values of PC1 and positive values of PC2, and cluster at the top left of the diagram. The dolomitic (Turonian) limestone in the Petra region (sample Jh limestone 60 outcrop, no. 44)) matches the calcareous (Turonian) rocks of the Northern Negev. The chalk from the tributary of Nahal HaRo'a (no. 4), however, is different and is closer to the Negev soils, with positive values of both PCs.

The sandstones from the Petra region show mostly high negative values of both PC parameters and are scattered over the fringes of the lower left corner of the diagram. They are chemically less uniform than the calcareous rocks, and very dissimilar from the Negev dust and soil samples. The sandstone bedrock of the triclinium on Jabal Farasha (sample JF site 124/1 rock, no. 38) is an outlier with a positive value of $\mathrm{PC} 1$, and a high negative value of $\mathrm{PC} 2$. It has elevated values of $\mathrm{Al}_{2} \mathrm{O}_{3}, \mathrm{Fe}_{2} \mathrm{O}_{3}$, $\mathrm{Cr}, \mathrm{Pb}, \mathrm{Sr}$, and $\mathrm{Y}$, and might represent a Paleozoic weathering crust. 
The hilltop ruin soils from the Petra region (the triclinium JF site 124/1 and Umm Saysaban, no. 67-71) are chemically similar and cluster in the lower center of the diagram, with negative values of PC2 and values of PC1 around 0. In contrast, samples from the sandstone terrace (samples Jh site 33, no. 60-66) scatter, suggesting contributions from different sources. However, the terrace on limestone (samples Jh limestone, no. 72-75), located in the upper center of the diagram, with values around 0 for PC1, and positive values of PC2, collected rather homogeneous material, different from the samples of the hilltop ruins. It seems, therefore, that the type of ruin matters less for the geochemical composition of archaeological soils in the Petra region, which is mainly associated with rocks. The rocks are surrounding the respective archaeological soils and dust samples, apparently 'pulling' them to the fringes of the matrix, probably depending on their content of weathered rock debris.

Therefore, it seems that the archeological soil and dust samples from the Petra region represent mixtures consisting of local material derived from the bedrocks, and of remote aeolian sediments similar to the dust and soil samples from the Northern Negev. Those samples that contain more calcareous silt are located closer to the Negev sample field, but all samples from the Petra region seem to be affected, by some degree, by their associated rocks.

\subsection{Pedogenesis}

Pedogenesis can affect grain sizes if it is sufficiently strong to alter minerals. The previous study on hilltop ruins [19] found that in-situ weathering of the archaeological soils was negligible, while aeolian sediments in the study areas comprise pre-weathered material. This study now includes archaeological soils of terraces to evaluate whether runoff irrigation and land use enhanced in-situ weathering. Parameters such as contents of extractable (pedogenic) iron, magnetic susceptibilities, and chemical indices are suited to characterize weathering intensities (Table 11, Table A11 at Appendix C).

In the Petra region, concentrations of oxalate-extractable iron Fe(o) are all below $0.6 \mathrm{mg} / \mathrm{g}$, suggesting negligible amounts of weakly crystalline oxidic iron compounds. Those of dithionite-extractable iron $\mathrm{Fe}(\mathrm{d})$ are $1.7-10.2 \mathrm{mg} / \mathrm{g}$, indicating more substantial occurrence of crystallized iron in all samples. With the exception of one sample from a silt-rich layer of the terrace on sandstone (Jh site $33100 \mathrm{~cm}$ ), amounts of extractable iron in the terraces $(3.9-10.2 \mathrm{mg} / \mathrm{g}$ ) do not exceed those of the hilltop ruin soils (which have 5.2 to $7.6 \mathrm{mg} / \mathrm{g}$ ). The soil of the monastery garden contains $5 \mathrm{mg} / \mathrm{g}$. All archaeological soils contain more $\mathrm{Fe}(\mathrm{d})$ than the natural hilltop soil on sandstone, which contains $2.5 \mathrm{mg} / \mathrm{g} \mathrm{Fe}(\mathrm{d})$. Most sandstones have minimal concentrations of Fe(d), except the bedrock of the triclinium on Jabal Farasha. The latter is an outlier with $52.2 \mathrm{mg} / \mathrm{g} \mathrm{Fe}(\mathrm{d})$, which supports that it represents an old weathering crust. In the Negev, concentrations of oxalate-extractable iron are below $0.6 \mathrm{mg} / \mathrm{g}$, too, while $\mathrm{Fe}(\mathrm{d})$ is between 1.5 and $5.0 \mathrm{mg} / \mathrm{g}$.

The Parker-index, which becomes smaller with increasing chemical alteration, mirrors the weathering of silicate rocks based on the proportions of alkali and alkaline earth metals [66]. In the Petra region, weathering intensity according to the Parker index seems to be more pronounced in soils affected by sandstone parent material. The natural sandstone soil has a Parker-index of 7, the hilltop ruin soils have values between 23 and 34, and runoff-irrigated terrace soils reach the highest values, ranging from 34 to 87 (which suggest they underwent the least chemical alteration). In the Negev, index values of the samples from the terraces (36 to 48) are slightly higher than those from the hilltop ruins (32 to 42 ).

The ratio of dithionite-extractable to total iron $\mathrm{Fe}(\mathrm{d} / \mathrm{t})$ can be a more useful parameter for assessing weathering intensity than absolute amounts of extractable iron, since it should reflect the degree to which the available iron has been oxidized [67]. This ratio shows small variations in soils of the Petra region, with numbers between 0.31 and 0.46 . It would suggest strongest weathering in the natural sandstone soil (0.46). In the Negev, values and variations are smaller and remain between 0.11 and 0.25. 
Table 11. Table summarizing indicators of pedogenesis of selected samples (full results in Table A11 in Appendix C). Fe(o) is oxalate-extractable iron, Fe(d) dithionite-extractable iron, $\mathrm{Fe}(\mathrm{d} / \mathrm{t})$ the ratio of dithionite-extractable to total iron, and Parker-index calculated according to [66]. $\chi$ is the weight-specific magnetic susceptibility, and $\chi_{\mathrm{FD}}$ the frequency-dependent change of susceptibility. The sample no. reflects the sample's number used in statistical analyses, ordered according to types of deposits (see Table A8 in the Appendix C).

\begin{tabular}{|c|c|c|c|c|c|c|c|c|c|c|c|}
\hline Sample Name & Sample No. & $\mathrm{Fe}(\mathrm{o})(\mathrm{mg} / \mathrm{g})$ & $\mathrm{Fe}(\mathrm{d})(\mathrm{mg} / \mathrm{g})$ & Fe $(d / t)$ & PARKER-index & $\mathrm{CaCO}_{3} \%$ & Sand \% & Silt \% & Clay \% & $X\left(\mathrm{~m}^{3} / \mathrm{kg}\right) \mathrm{E}-8$ & $\mathrm{X}_{\mathrm{FD}} \%$ \\
\hline \multicolumn{12}{|c|}{ Petra Region } \\
\hline \multicolumn{12}{|c|}{ Natural Hilltop Soil on Sandstone Plateau: Protic Arenosol (Ochric) } \\
\hline Sandplateau 1 & 47 & 0.1 & 2.5 & 0.41 & 7 & 2 & 80 & 12 & 8 & 20.0 & -4.3 \\
\hline Sandplateau 4 & 50 & 0.0 & 1.7 & 0.42 & 2 & 0 & 82 & 8 & 10 & 11.0 & 3.6 \\
\hline Sandplateau Stein (bedrock) & 39 & 0.0 & 0.1 & 0.20 & 1 & 0 & 89 & 7 & 4 & -0.2 & \\
\hline \multicolumn{12}{|c|}{ Jabal Farasha Triclinium Hilltop Ruin: Calcaric Leptosol (Protic) } \\
\hline JF site $124 / 15 \mathrm{~cm}$ & 67 & 0.2 & 7.5 & 0.44 & 34 & 13 & 51 & 36 & 13 & 32.0 & 5.3 \\
\hline JF site $124 / 1$ bedrock & 38 & 0.2 & 52.2 & 0.63 & 10 & 2 & 60 & 18 & 22 & 7.0 & 14.4 \\
\hline \multicolumn{12}{|c|}{ Umm Saysaban Hilltop Ruin: Calcaric Leptosol (Protic) } \\
\hline Umm Saysaban $5 \mathrm{~cm}$ & 70 & 0.2 & 5.6 & 0.34 & 31 & 12 & 51 & 35 & 14 & 28.0 & 6.6 \\
\hline \multicolumn{12}{|c|}{ Buried Early Holocene Paleosols: Cambic Calcisol (Hypocalcic) (below Shkarat Msaied Ruins); Cambisol (Protocalcic) (below Abu Suwwan Ruins) } \\
\hline Shakarat Msaid 1 & 59 & 0.3 & 4.4 & 0.31 & 36 & 15 & 52 & 26 & 22 & 22.0 & 5.1 \\
\hline Abu Suwwan below nw 65 & 58 & 0.1 & 4.0 & 0.39 & 34 & 18 & 62 & 23 & 15 & 82.0 & 6.3 \\
\hline \multicolumn{12}{|c|}{ Monastery Garden of Jabal Haroun: Protic Arenosol (Alcalic, Ochric) } \\
\hline Jh Site $230 \mathrm{~cm}$ & 53 & 0.1 & 5.0 & 0.43 & 14 & 3 & 80 & 7 & 13 & 15.4 & 18.7 \\
\hline \multicolumn{12}{|c|}{ Terrace on Dolomitic Limestone (Jh site 60): Protic Colluvic Calcaric Regosol } \\
\hline Jh limestone 20 & 73 & 0.3 & 5.0 & 0.33 & 87 & 55 & 32 & 47 & 21 & 28.9 & 1.6 \\
\hline Jh limestone 60 & 75 & 0.2 & 4.8 & 0.32 & 85 & 55 & 30 & 43 & 27 & 24.1 & 1.1 \\
\hline \multicolumn{12}{|c|}{ Terrace on Sandstone (Jh site 33): Protic Calcaric Arenosol (Colluvic) over Protic Colluvic Calcaric Regosol } \\
\hline Jh site 3310 & 60 & 0.2 & 3.9 & 0.34 & 67 & 39 & 66 & 27 & 7 & 20.6 & 1.4 \\
\hline Jh site 3330 & 61 & 0.1 & 4.2 & 0.41 & 38 & 11 & 73 & 15 & 12 & 7.7 & -2.6 \\
\hline Jh site 3350 & 62 & 0.1 & 4.0 & 0.40 & 34 & 7 & 74 & 15 & 11 & 6.3 & -4.8 \\
\hline Jh site 3375 & 63 & 0.1 & 6.8 & 0.33 & 54 & 15 & 32 & 41 & 27 & 16.2 & -0.1 \\
\hline Jh site 33100 & 64 & 0.2 & 10.2 & 0.37 & 60 & 23 & 16 & $\begin{array}{l}11 \\
45\end{array}$ & 39 & 18.4 & $\begin{array}{l}-0.1 \\
-13.6\end{array}$ \\
\hline Jh site 33130 & $\begin{array}{l}04 \\
65\end{array}$ & 0.2 & $\begin{array}{l}10.2 \\
7.3\end{array}$ & 0.35 & $\begin{array}{l}60 \\
62\end{array}$ & 28 & $\begin{array}{l}10 \\
31\end{array}$ & 36 & 33 & $\begin{array}{l}10.4 \\
18.9\end{array}$ & $\begin{array}{c}-15.0 \\
0.9\end{array}$ \\
\hline Jh site 33150 & 66 & 0.2 & 5.9 & 0.37 & 54 & 24 & 59 & 23 & 18 & 15.5 & 0.6 \\
\hline \multicolumn{12}{|c|}{ Negev } \\
\hline \multicolumn{12}{|c|}{ Paleosol in Loessial Apron of tributary of Nahal HaRo'a: Cambic Calcisol (Siltic) } \\
\hline NH-LA-30cm & 6 & 0.4 & 4.6 & 0.20 & 66 & 27 & 27 & 58 & 15 & 61.1 & 2.8 \\
\hline \multicolumn{12}{|c|}{ Western Wadi Terrace 3: Protic Colluvic Calcaric Regosol (Siltic) } \\
\hline HH-WW-T3-20cm & 13 & 0.5 & 5.0 & 0.23 & 74 & 36 & 33 & 45 & 22 & 44.5 & 4.9 \\
\hline HH-WW-T3-70cm & 15 & 0.4 & 4.6 & 0.25 & 80 & 48 & 30 & 55 & 15 & 40.5 & 5.2 \\
\hline \multicolumn{12}{|c|}{ Hilltop Ruin Overlooking the Western Wadi: Calcaric Leptosol (Protic. Siltic) } \\
\hline HH-WW R1 10 & 22 & 0.2 & 2.5 & 0.14 & 75 & 35 & 42 & 45 & 13 & 43.6 & 3.7 \\
\hline \multicolumn{12}{|c|}{ Hilltop Ruin Overlooking the Central Wadi: Protic Calcaric Regosol (Siltic) over Cambic Calcisol (Siltic) } \\
\hline HH-CW-Ruin 10 & 24 & 0.5 & 4.3 & 0.20 & 82 & 40 & 22 & 53 & 25 & 64.0 & 6.1 \\
\hline HH-CW-Ruin 50 & 26 & 0.5 & 3.1 & 0.17 & 85 & 40 & 41 & 47 & 12 & 74.0 & 7.6 \\
\hline HH-CW-Ruin-90 & 29 & 0.2 & 2.1 & 0.20 & 69 & 27 & 27 & 51 & 22 & 55.0 & 2.9 \\
\hline
\end{tabular}


Plotting the Parker-index against the ratio of $\mathrm{Fe}(\mathrm{d} / \mathrm{t})$, a negative correlation can be observed in the Petra region, i.e., stronger chemical weathering corresponds to higher ratios of dithionite-extractable to total iron $\mathrm{Fe}(\mathrm{d} / \mathrm{t})$ (Figure 14a). However, there is no connection between $\mathrm{Fe}(\mathrm{d})$ and $\mathrm{Fe}(\mathrm{d} / \mathrm{t})$ (Figure 14b). This suggests that varying amounts of pre-weathered minerals are inherited from diverse sources in the Petra region, in particular, in those soils with a large contribution from the sandstones. However, a positive correlation of silt contents with total amounts of extractable iron Fe(d) (Figure 14c), and a negative correlation with the sand fraction (Figure 14d) indicate that part of the extractable iron is bound to a calcareous silt fraction.

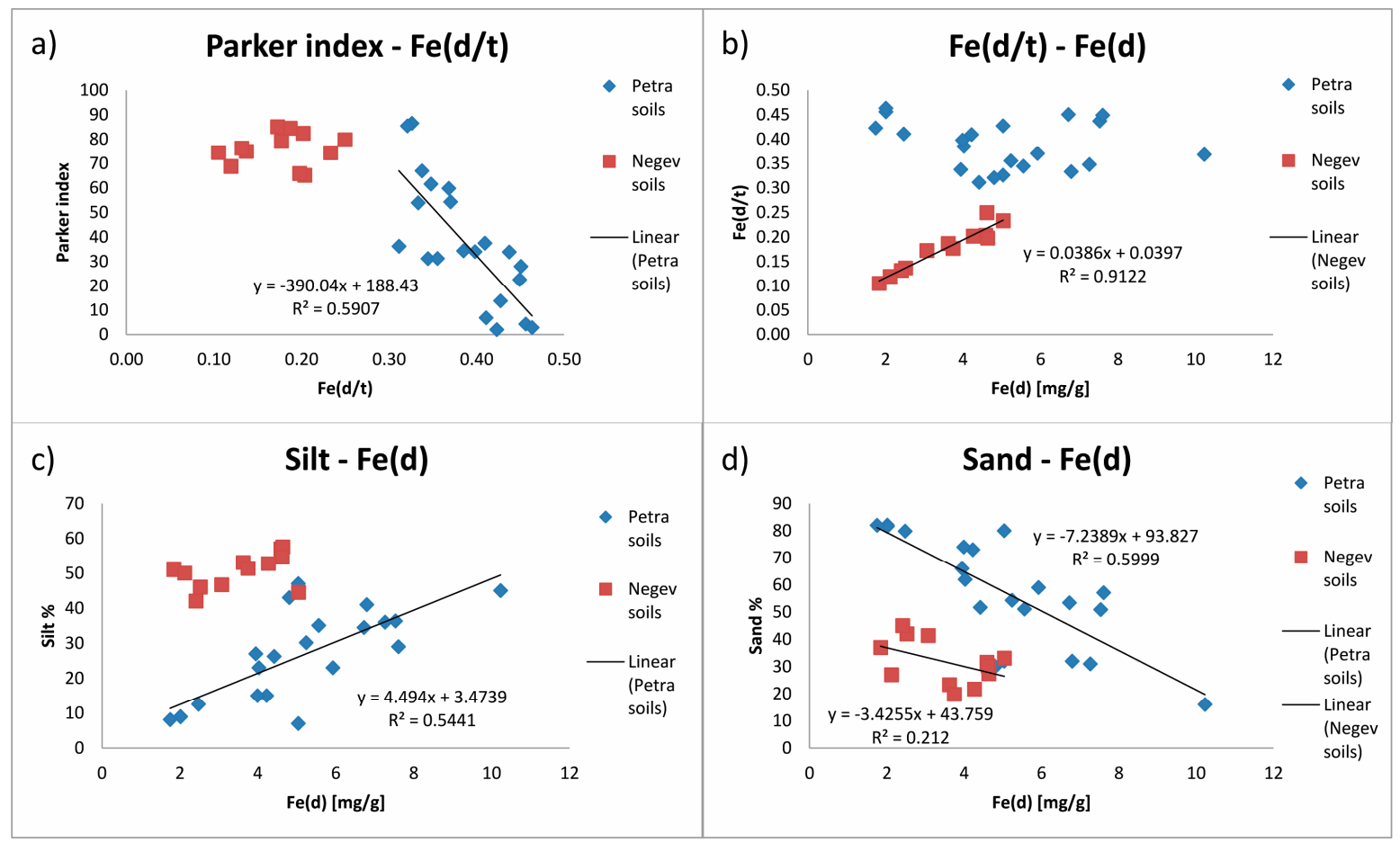

Figure 14. (a) Correlation of Parker-index and ratio $\mathrm{Fe}(\mathrm{d} / \mathrm{t})$ of dithionite-extractable to total iron for soils of the Petra region (blue diamonds) and Negev soils (red squares); (b) relation of Fe(d) to ratio of dithionite-extractable to total iron $\mathrm{Fe}(\mathrm{d} / \mathrm{t}$ ) for soils of the Petra region (blue diamonds) and Negev soils (red squares); (c) positive correlation of silt contents to Fe(d) of Petra region (blue diamonds) and Negev soils (red squares); (d) negative correlation of sand contents to Fe(d) of Petra region (blue diamonds) and Negev soils (red squares). Rock samples were not included in the diagrams.

In contrast, a strong linear relationship between $\mathrm{Fe}(\mathrm{d})$ and $\mathrm{Fe}(\mathrm{d} / \mathrm{t})$ can be observed in the Negev (Figure 14b), but no connection is observed between the Parker-index and Fe(d/t) (Figure 14a). As well, there is no connection between $\mathrm{Fe}(\mathrm{d})$ and grain sizes. This points to a rather homogeneous presence of iron that is not connected with chemical weathering of the substrates.

Magnetic susceptibility $\chi$ shows a positive correlation with silt contents in both areas $\left(R^{2} 0.6\right.$ for the whole dataset) (not shown in figure). It suggests that the minerals which contribute the magnetic signal are mainly of aeolian origin. However, there is no connection of $\chi$ with the total amounts of extractable iron, although the $\mathrm{Fe}(\mathrm{d} / \mathrm{t})$-ratio shows some correspondence $\left(\mathrm{R}^{2} 0.54\right)$. This adds to the rather chaotic pattern of weathering intensities, suggesting diverse sources of magnetic minerals, and further indicating a negligible role of in-situ weathering. The outlier of the paleosol below the site of Abu Suwwan (sample no. 58) can be explained by the addition of burnt ash, which can lead to high susceptibilies [61]. 
In summary, weathering intensity parameters are partly contradictory and no consistent pattern or role of terrace irrigation can be observed. This seems due to mixtures of diverse pre-weathered materials from various sources, while in-situ weathering is negligible.

\subsection{Statistical Modeling of Sample Composition}

In order to test the possibility of predictions of the deposit type and investigation region with the collected parameters, we performed a random forest classification [57]. For this, samples were classified as different types of deposits. These are in the first place: Archaeological Soils with their sub-types Hilltop Ruin Soil, Terrace Soil, and Cistern Cleanout Sediment, and Current Dust and Rock References. In addition, we sub-classified dust and rock samples according to their investigation regions, and defined the classes of 'Petra Natural Sandstone Soil', 'Negev Pleistocene Paleosol', and 'Petra Pleistocene Paleosol'. The monastery garden was assumed to represent a 'Petra Natural Sandstone Soil'. A full list of all samples with their respective assignments is presented in Table A8 in Appendix C. Modeling included the complete dataset with all parameters that were available for all samples (grain sizes and derived parameters, element concentrations and derived ratios, magnetic susceptibilities). Smaller datasets with less parameters produced higher errors.

The achieved result had an overall classification error of only $10.71 \%$. The same error was present when summarizing all sub-types of archaeological soils to one respective class, but with a slightly different distribution of the erroneous classification of samples. Separating archaeological soils according to investigation areas led to higher errors. The same was true when summarizing rock and dust samples from the two regions. This supports the observation that archaeological soils are rather similar, although rocks and current dust storm sediments from the two investigation areas are characterized by different properties.

The response variable was the deposit type, consisting of the classes Negev Rock Reference $(n=4)$, Negev Pleistocene Paleosol $(n=5)$, Terrace Soil $(n=21)$, Cistern Sediment $(n=4)$, Hilltop Ruin Soils $(n=11)$, Negev Dust $(n=8)$, Petra Rock Reference $(n=7)$, Petra Natural Sandstone Soil $(n=13)$, Petra Pleistocene Paleosol ( $n=2)$, and Petra Dust $(n=9)$. We predicted these classes on the basis of explanatory variables from grain sizes, geochemical parameters, and magnetic susceptibilities, and were able to model the classes Negev Dust, Petra Dust, and Terrace Soil with $100 \%$ accuracy. The classes Petra natural Sandstone Soil and Hilltop Ruin Soil were modeled with errors of $7.7 \%$ and 9\%, meaning that one out of the 16 Natural Sandstone Soil samples was incorrectly assigned to the class Petra Pleistocene Paleosol, and one of 11 Hilltop Ruin Soil samples was assigned to Terrace Soil. The class Petra Rock Reference showed an error of $14 \%$, with one sample incorrectly assigned to Negev Rock Reference. Negev Pleistocene Paleosol showed an error of $20 \%$, with one out of four samples falsely allocated (to Terrace Soil). Negev Rock Reference showed an error of $25 \%$ with one out of four samples assigned to Terrace soils. The Cistern Sediment was difficult to model with an error of $50 \%$, and two of four samples assigned to Terrace Soils.

When summarizing the different ruin types as one class of 'Archaeological Soil', classification of this type was $100 \%$ correct, but the total error of $10.71 \%$ remained unchanged since additional paleosol and rock samples were classified incorrectly. Dust samples from the two regions were always modeled without error.

Table 12 summarizes the wrongly assigned samples. It can be discerned that erroneous classifications refer mainly to special samples, or transitional samples that are difficult to assign to a certain group. 
Table 12. List of samples with erroneous type prediction by the statistical model of the random forest approach. Modeling was based on the full dataset including grain sizes and connected statistical parameters such as main mode and mean grain size, element concentrations and derived ratios, and magnetic susceptibilities. The full list of samples with assigned types of deposits is presented in Table A8 in Appendix C.

\begin{tabular}{|c|c|c|c|}
\hline Sample & Original Classification & Predicted Classification & Comment \\
\hline Jh limestone 60 outcrop & Petra Rock Reference & Negev Rock Reference & Turonian dolomitic limestone \\
\hline Beidha Fan & Petra Nat. Sandst. Soil & Petra Pleistocene & High concentrations of $\mathrm{CaO}, \mathrm{K}_{2} \mathrm{O}, \mathrm{Rb}$ \\
\hline Abu Suwwan below nw 65 & Petra Pleistocene & Petra Nat. Sandst. Soil & \multirow{2}{*}{$\begin{array}{c}\text { Paleosols show intermediate } \\
\text { properties among natural and } \\
\text { archaeological soils }\end{array}$} \\
\hline Shakarat Msaid 1 & Petra Pleistocene & Terrace Soil & \\
\hline HH-CW-Tur-Paleo & Negev Rock & Terrace Soil & Turonian paleosol \\
\hline HH-WW-Cistern1-Cleanout1-120 & Cistern Sediment & Terrace Soil & \multirow{2}{*}{$\begin{array}{l}\text { Role of settling tank for correctly } \\
\text { modeled cistern samples? }(\rightarrow \text { no } \\
\text { particles }>\text { fine sand })\end{array}$} \\
\hline HH-WW-Cistern1-Cleanout1-160 & Cistern Sediment & Terrace Soil & \\
\hline HH-CW-Ruin 10 & Hilltop Ruin & Terrace Soil & Recycled terrace material in hilltop? \\
\hline HH-CW-Ruin 90 & Negev Pleistocene & Terrace Soil & $\begin{array}{c}\text { Diffuse boundary to paleosol below } \\
\text { ruin }\end{array}$ \\
\hline
\end{tabular}

With regard to the variable importance, model results were predominantly achieved by grain size parameters such as contents of fine clay, coarse silt, total clay, medium sand, and main mode, as well as by $\mathrm{K} / \mathrm{Al}_{2} \mathrm{O}_{3}$-ratio and concentrations of $\mathrm{K}_{2} \mathrm{O}, \mathrm{Zn}, \mathrm{TiO}_{2}, \mathrm{Ba}, \mathrm{Al}_{2} \mathrm{O}_{3}, \mathrm{Rb}$, and Sr. Weathering indices such as index of chemical alteration CIA A [68] and Parker-index [66] were of some importance, too, but most geochemical elements had only minor explanatory power. It should be mentioned that the importance of fine clay could be overestimated due to an analytical issue connected with laser measurements of dust samples, because fine clay could not be detected with the laser, but was found in Sedigraph analysis [19].

Among the well-distinguished archaeological soils of the two investigation regions, only the Cistern Cleanout sediment was difficult to model, which could be connected with the possible use of a settling tank that removed particles larger than fine sand in the younger sediments, and the small number of samples. As well, paleosols in the Petra region seem difficult to separate from archaeological soils and current natural soils. Rocks can well be distinguished, including the Turonian dolomitic limestone from the Petra region that is similar to the contemporaneous Negev rocks.

It can be summarized that a certain grain size distribution including most particle size classes, plus rather high concentrations of various major and trace elements, seem characteristic of most archaeological soils and their sub-types, allowing distinction from dust, rocks, and current soils with high certainty. Paleosols near Petra, however, take a transitional position between archaeological soils and natural current soils. This could be connected with higher element concentrations derived from long-term dust input during soil formation, while their grain sizes seem largely inherited from weathered sandstones.

\section{Discussion}

\subsection{Role of the Types of Archaeological Ruins}

With the exception of the monastery garden on Jabal Haroun, archaeological soils in the different ruin types in the two study areas are similar. This was already suggested by the field identification of most substrates as calcareous (silt) loam. Archaeological soils seem in general to consist of complex mixtures including most grain size classes, rather high shares of silt and fine sand, and elevated concentrations of various major and trace elements. This is supported by the successful statistical modeling of archaological soils as one characteristic deposit class in both study regions, which assigns only the monastery garden to the group of natural soils in the Petra region. The total error of $10.71 \%$ is small, referring mainly to transitional samples difficult to assign to pre-defined deposit classes. 
It suggests that the composition of archaological soils reflects mainly aeolian sediment sources and deposition processes, whereas the fluvial re-distribution to terraces or cisterns plays a subordinate role.

The different ruin types are, however, characterized by divergent soil structures. All hilltop ruin soils have V-horizons (or vesicular layers) and clast pavements at the surface, which could not be identified in the other ruin types. Formation of banded layers seems connected with sedimentation in abandoned terraces, while plowing of agriculturally used soils apparently led to obliteration of sedimentary structures.

These structural differences seem to some degree connected with minor, but apparently systematic, variations of substrate composition. Although some samples where incorrectly assigned in further statistical modeling of the different ruin types as characteristic deposit classes, the total error of this model was identical to the one summarizing all archaeological soils to one class. Incorrect assignments were mostly related to the cistern cleanout sediments. This could be connected with a small number of samples, or with the possible presence of a settling tank that might have led to exclude particles coarser than fine sand.

\subsection{Role of Rocks}

A contribution of sandstones to natural soil formation in the Petra region is indicated by high medium sand contents with a main mode of $415 \mu \mathrm{m}$. These are present in the natural hilltop soil on sandstone and in the paleosols that were preserved below ruins, probably due to physical disintegration of rocks and the presence of sand-trapping vegetation [19]. With regard to the archaeological soils, however, high medium sand content is only present in the monastery garden on Jabal Haroun. A sandy layer in the terrace on sandstone, in contrast, contains mainly fine sand with a main mode of $132 \mu \mathrm{m}$, which is the dominating sand fraction of all other archaeological soils. The exceptional profile in the monastery garden is located on a sandstone plateau with limited fluvial catchment, but surrounded by weathered sandstone cliffs. This suggests that not fluvial transport, but short-range aeolian contribution from the rocks is the main source of medium sand.

The contribution from sandstones seems minimal at the terrace on dolomitic limestone. There, grain sizes are similar to archaeological soils in the Negev, which suggests that aeolian sediments from similar sources and/or comparable transport and deposition processes settle in the Petra region. Chemical properties of archaeological soils in the Negev and the limestone terrace in the Petra region appear rather similar and suggest similar (remote?) dust sources. However, compared with averages of the Negev archaeological soils, sediments on the dolomitic limestone terrace are enriched by $\mathrm{CaO}$ and $\mathrm{MgO}$ ( $25 \%$ and $5 \%$ vs. $\sim 21$ and $\sim 2 \%$ ), and depleted by all other major and trace elements (20-70\% less than average concentrations in the Negev; smaller depletions of $20-30 \%$ are observed in the silt\&clay fractions of sample Jh limestone 60). This is attributed to contribution from the surrounding weathered dolomitic limestone outcrops, as reflected by the relatively high $\mathrm{MgO}$ - and $\mathrm{CaO}-$ contents (independent from grain size). Such a contribution from this rock is evident in the adjacent terrace on sandstone, too.

Element concentrations suggest that all archaeological soils in the Petra region are to some degree affected by their surrounding rocks. The sandstones play not only a significant role for chemical properties of archaeological soils, but also for the composition of dry dust. Most dry dust samples are very similar to sandstones, but dust associated with precipitation contains more silt and is rich in $\mathrm{CaCO}_{3}$, which makes it more similar to the archaeological soils. In contrast, such contributions from the rocks cannot be identified in the Northern Negev. No chemical distinction between dust storms and archaeological soils is possible in the Negev, and Negev rocks are a negligible sediment source.

This is probably related to rock hardness and crusts, as most calcareous rocks in the Negev are very hard, and locally sealed by biological and physical crusts $[49,50]$. In the Petra region, in contrast, sandstones weather rapidly, forming characteristic structures related to wind erosion. This includes aeolian erosion of clays, which were found to migrate with water during weathering of sandstones. They accumulate near the surface, weaken the rocks by shrink-swell processes, and lead to flaking of 
rock pieces that are blown away [58]. The presence of crusts in the Petra region appeared scattered [19], and the dolomitic limestone was friable and could be cut with a spade.

\subsection{Aeolian and Fluvial Transport Processes}

While deflation from rock outcrops, and probably also fans, is evident in the Petra region, it seems rather irrelevant for the archaeological soils. These form by accretionary processes and do not show a depletion of fine fractions, but on the contrary, a relative enrichment of silt. Even the natural soil on sandstone is characterized by higher silt and clay contents towards the top of the soil profile, which reflect increasing dust deposition on the weathered sandstone.

It is notable that medium and coarse sand are missing in the lower, silt-rich layer of the terrace on sandstone (Jh site 33) near Petra. This layer contains similar amounts of silt and fine sand as the nearby terrace on dolomitic limestone (Jh site 60). It was apparently deposited during the time of terrace cultivation and maintenance. The absence of medium and coarse sand suggests effective flood control, and that aeolian movement of such sand grains does mostly not cross distances of more than $100 \mathrm{~m}$. Upon abandonment, when terraces on top of the sandstone slope were eroded, fluvial deposition of sand resumed in the terrace on sandstone. However, even the upper part of the sandstone terrace profile is dominated by fine sand, apparently aeolian, in contrast to the samples from alluvial fans, which consist mainly of medium sand. This adds support that fluvial processes play a minor role for the composition of the studied terrace substrates, while aeolian sedimentation of medium sand seems to be limited to the immediate vicinity of sandstones such as at the monastery garden of Jabal Haroun.

In the Negev, terraces are dominated by fine sand and silt, similarly to the other archaeological soils. This indicates a predominance of aeolian processes for the composition of all investigated terrace substrates.

Fluvial processes such as runoff irrigation of the terraces seem to play a role for the speed of sediment aggradation, but not the composition of the substrates. There is no connection between fluvial catchment size and sediment properties. Aeolian processes and sources dominate; therefore, the composition and primary deposition of sediments in all archaeological soils, which are only re-distributed by runoff.

This is supported by the current dust samples. Due to technical reasons, the dust collector at the summit of Jabal Haroun had to be placed on a secluded rock outrop, which implied immediate neighborhood to a sandstone cliff. It is reflected by high amounts of medium sand, similar to the monastery garden.

\subsection{Pedogenesis}

Extractable iron, chemical weathering indices, and magnetic susceptibilities are sensible indicators of weathering processes, which are suited to characterize in-situ pedogenesis of soils in semi-arid regions [22,64]. However, Lucke et al. [19] found no in-situ weathering in hilltop ruins, but that varying amounts of pre-weathered iron were inherited from diverse sources including the sandstone rocks in the Petra region. This pattern is confirmed by the additional samples of the current study, and no effect of runoff irrigation and land use on weathering can be discerned in the terrace soils.

The presence of burnt ash in the archaeological soils, macroscopically observed in the paleosol below the site of Abu Suwwan and in the monastery garden (Jh site 2), is associated with elevated magnetic susceptibility only in case of the paleosol at Abu Suwwan. Apart from that, the ash additions seem not reflected by any parameter. This is in line with earlier studies [61] and probably connected with the low mass of the ash.

Extractable iron $\mathrm{Fe}(\mathrm{d})$ in the Petra region is partly connected with calcareous silt, which also contributes the largest part of the magnetic susceptibility of the soils. This suggests that part of the iron and magnetic minerals were supplied by remote dust sources.

Extractable iron in the Northern Negev is lower than in the Petra region, and geochemical indices suggest lower weathering intensities, but higher magnetic susceptibilities. There is a strong positive 
correlation of $\mathrm{Fe}(\mathrm{d})$ and $\mathrm{Fe}(\mathrm{d} / \mathrm{t})$, whereas values of weathering indices are rather uniform with no clear tendencies. This suggests a homogeneous (aeolian) source of iron in the Negev. With regard to the Negev paleosols, indices of weathering intensity suggest only minimally stronger pedogenesis. This is associated with slightly reduced contents of $\mathrm{CaCO}_{3}$, which indicates some leaching that was probably connected with precipitation of the secondary carbonate nodules. In this context, remains of Pleistocene paleosols were found at various locations, such as below the cistern cleanout spoil, or below the terrace in the tributary of Nahal HaRo'a. The remains represent wider distribution of these soils during antiquity, which were eroded and recycled into the archaeological soils [30]. This could explain some of the variations in the profiles, as concentrations of $\mathrm{Fe}(\mathrm{d})$ in the hilltop ruin at the central wadi, or lower $\mathrm{CaCO}_{3}$-contents of the terrace soil in the tributary of Nahal HaRo'a.

\subsection{Land Use Effects}

Higher conductivities and slightly elevated clay contents can be observed in the cistern cleanout samples and layers of terrace soils which were probably cultivated. In the Petra region, a linear positive correlation $\left(R^{2} 0.54\right)$ can be observed between clay contents and conductivity, but not in the Negev. Higher conductivities could result from prolonged agricultural irrigation if drainage was bad. However, the cistern cleanout spoil suggests that irrigation is an unlikely reason of elevated conductivities.

In contrast, a sedimentary origin of the elevated conductivities and slightly higher clay contents seems probable, connected with puddles of temporary standing water. Kidron et al. [69] reported that dust samples gathered in wet collectors were characterized by elevated clay contents. Runoff water gathered in the cisterns, and temporary standing water may have formed behind intact terraces walls, infiltrating only slowly into the soil. Even today, such water puddles can form after major rain events in terrace remains-see Figure 3 in Bruins et al. [50] (p. 95). Evaporation from such puddles could lead to higher salt concentrations. Thus temporary standing water that gathered behind well-maintained walls could explain an increase of clay contents and conductivities.

High concentrations of $\mathrm{P}_{2} \mathrm{O}_{5}$, such as in the hilltop ruin at the central wadi, are interpreted as a marker of organic refuse. Similarly elevated phosphorus concentrations have been reported from hilltop ruins in Northern Jordan [61,64]. Debris from ruined settlements could have been subject to short-range aeolian deposition onto associated hilltops, which was indicated by the single-grain structure and low bulk density of $0.9 \mathrm{~g} / \mathrm{cm}^{3}$ (similar to the hilltop ruin soils of this study [61]). It seems likely that local dust sources comprise fine fractions of nearby ruin debris and refuse. The higher concentrations of $\mathrm{P}_{2} \mathrm{O}_{5}$ in the hilltop ruin at the central wadi in the Negev may thus indicate a dust contribution from the adjacent small group of round and rectangular structures, which apparently resemble remains of a small ruined settlement.

A re-mobilization of terrace soils into the aeolian system could be possible due disturbances such as plowing, potentially inducing deflation (possibly preferentially of silt), but no evidence was detected in the studied profiles. Only statistical modeling of deposit types gave a hint in this direction, as the uppermost sample from the hilltop ruin soil at the central wadi in Horvat Haluqim was modeled to represent a terrace soil. This sample was characterized by strongly elevated sedimentation rate and platy structure, probably due to collapsed vesicular layers resulting from Bedouin re-use of the place for campfires [19]. This lets it seem possible that land use can have a local effect on dust emission and deposition, but which seems of minor importance in the studied profiles.

Collection of dust-laden runoff water may amplify sediment accretion, which seems connected with the catchment areas. Terrace 3 at Horvat Haluqim is estimated to have collected 3.5 times more sediment than the nearby hilltop ruin at the central wadi, while the limestone terrace in the Petra region, with similar catchment size, seems to have gathered two times more sediment than the nearby hilltop ruin at Jabal Farasha. However, a direct comparison of these terraces is not possible, as Terrace 3 is apparently still collecting sediments. In contrast, sedimentation at the limestone terrace might have stopped or been reduced in the past as its wall was not built higher, but filled completely. 


\subsection{Effects of Proximity to Local Sediment Sources and Dust Fixation Processes}

In the Petra region, the archaeological soils do not simply represent the composition of aeolian materials collected in the dust samplers, but other factors must be involved in their formation. Mean annual sedimentation rates on the triclinium on Jabal Farasha suggest that only $20-50 \%$ of the material that could be collected in current dust samplers at Jabal Haroun settled there (see detailed calculation of sedimentation rates in Appendix D.5.). However, sedimentation rates at the monastery garden on Jabal Haroun are similar to the amounts collected in current samplers. This seems due to a contribution from nearby sandstone cliffs: proximity to such local sources leads to higher supply of sediment, in this case, rich in medium sand. In addition, the agricultural use of the monastery garden, probably with cistern-based irrigation, could have led to stronger presence of vegetation, which has been found to favor the trapping of sand [70].

It seems possible that aeolian sedimentation in the dust collectors does not fully mirror natural processes, but that part of the collected dust is in reality remobilized unless trapped by sediment-fixing agents such as marble fills. Surface crusts and clast pavements were found to fix dust and to favor the settling of silt and clay [71-74]. The role of crusts as sediment-fixing agents seems more pronounced in the hilltop ruins, as already indicated by the presence of V-horizons, leading to a preferential fixation of silt and clay and thus higher concentrations of associated elements. Silt and clay fractions of hilltop ruins in the Petra region show enrichment of all major elements (by 30-100\%) except $\mathrm{SiO}_{2}$, and all trace elements (by 50-120\%), which suggest that silt rich in kaolinite and iron preferentially accumulates. This effect was less pronounced in the terrace soils, where silt and clay fractions showed enrichment by major elements except $\mathrm{SiO}_{2}$ by $5-25 \%$, and of trace elements by $5-100 \%$. This seems to reflect a less significant role of surface crusts in the terraces, resulting from plowing and fluvial accumulation.

The dust sampler at the foot of Jabal Haroun is located close to a ridge of fine sediments containing rizoliths, possibly representing Pleistocene slackwater deposits [19]. As two out of three dust samples from the foot of the mountain are characterized by rather high contents of $\mathrm{CaCO}_{3}$, Lucke et al. [19] concluded that these were probably derived from the rizoliths. However, a re-interpretation seems necessary in light of the now evident role of local sediment sources in the Petra region. These two samples were associated with lower wind speeds (see Lucke et al. [19], p. 19, Table 6), while the sample associated with high wind speed showed small contents of $\mathrm{CaCO}_{3}$, high share of medium sand, and very similar properties to the dry dust samples from the summit of Jabal Haroun. Therefore, this sample might in fact represent a contribution from the nearby ridge of fine sediments, while the two samples with higher $\mathrm{CaCO}_{3}$-contents could represent aeolian sediments less affected by proximity to sandstones.

Annual sedimentation rates of current dust samplers were highly variable, which can be illustrated with a maximum of sediment deposition at a one-time event (probably a dust devil) on the summit of Jabal Haroun on 5 August 2017, exceeding total sedimentation during the rest of the year. Therefore, average sedimentation rates must be treated with caution. Nevertheless, the combined results from dust samples and archaeological soils in the Petra region suggest that variability of the amount and composition of dust largely depends on proximity to rock sources. In addition, clast pavements and crusts favor fixation of silt and clay, leading to a relative decrease of the sand fraction in the archaeological soils.

In contrast, effects of dust emissions from rock outcrops play no role in the Negev, and dust storms are already dominated by silt. Therefore, crusts and clast pavements do not much affect substrate composition by processes of dust fixation. Grain size composition of archaeological soils in the Negev differs to some degree from current dust storm sediments, which show higher silt and smaller sand contents. This is mostly connected with the coarse and medium sand fractions, which could resemble debris in the archaeological soils, or be missing in our Negev dust sampler due to its position $\sim 80 \mathrm{~cm}$ above ground.

Local sediment sources other than rocks, however, seem to play a role for current dust. A contribution of local sources to dustfall in the Negev has been reported by various other studies [75-77]. 
It was estimated to comprise around 50\% of dust deposition in the Negev, gradually decreasing towards the north of Israel, and largely depending on the erodibility of potential sources [78].

Current annual sedimentation rates at Sede Boker were found to be $110-178 \mathrm{~g} \mathrm{~m}^{-2} \mathrm{a}^{-1}$ ( $\sim 122.4 \mathrm{~g} \mathrm{~m}^{-2} \mathrm{a}^{-1}$ on hilltops) [69]. This agrees with average deposition rates in the hilltop ruin of the central wadi at Horvat Haluqim [19], which we recalculate to 116-180 $\mathrm{g} \mathrm{m}^{-2} \mathrm{a}^{-1}$ (applying the skeleton-free bulk density of $0.9 \mathrm{~g} / \mathrm{cm}^{3}$ and average sedimentation of $0.13-0.2 \mathrm{~mm} \mathrm{a}^{-1}$ ). Dust accumulation processes in hilltop ruin soils in the Negev seem thus to be well represented by sedimentation in dry standard dust collectors filled with glass marbles. It is worth noting that these Late Holocene mean annual sedimentation rates exceed (the relatively small) Pleistocene dust accretion in the natural hilltop loess profile of Mount Harif in the southern Central Negev (0.02-0.058 $\mathrm{mm} \mathrm{a}^{-1}$, or 30-87 $\mathrm{g} \mathrm{m}^{-2} \mathrm{a}^{-1}$ according to [12]).

The importance of local sources for dust deposition in the Levant could in general have been underestimated due to a focus on well-rounded or subangular quartz grains indicating aeolian abrasion $[61,64,79]$. In this context, a scanning electron microscope (SEM) study of the above mentioned hilltop ruin soil in Northern Jordan found a soil matrix consisting mainly of microfossils from chalk, angular calcite pieces, and bone and pottery fragments [61]. It was interpreted as remains of in-stu disintegrated plaster and debris, but this material could in fact have been subject to short-range aeolian motion. This would suggest that aeolian deposition in hilltop ruins is a common process in arid and semi-arid areas, which may have supplied approximately $20 \mathrm{~cm}$ of hilltop ruin soil cover during the past 2000 years in Northern Jordan [19,61].

\subsection{Role of Precipitation}

Only one sample deposited with precipitation was available from the Negev. In contrast to the proposal of Issar and Bruins [7], who suggested that clay might have increasingly settled during periods of more precipitation, it is not characterized by elevated clay contents, but higher silt and smaller sand contents. Its geochemical composition, however, is very different from the other dust samples and seems to include a contribution from leached Mediterranean soils (Terra rossa).

Two dust samples in the Petra region were associated with snow and rain, and show a very different composition than the other dust samples from the mountain summit. They are characterized by much higher contents of silt and $\mathrm{CaCO}_{3}$, and the clay fraction seems slightly elevated in the snow dust sample. With regard to chemical composition, these samples are depleted in $\mathrm{SiO}_{2}$, but enriched by all other major and trace elements, more similar to the ruin soils and mostly showing approximately twice the concentrations of dry dust samples. This suggests that these elements, as well as extractable iron and magnetic susceptibilities, are bound to a calcareous silt fraction that settles to a higher degree when associated with precipitation. This seems a similarity to the Negev rain dust sample.

One aspect of precipitation could be the wetting of rock surfaces, as well as the activation of biological crusts, as these could minimize local dust emissions. Such a process was observed in the Negev, where rainfall had a postponed effect: dust concentration, and the velocity and amount of deposition, were significantly reduced during a dry season if preceded by a wet season with frequent rains [77] (pp. 241-245). This suggests that local sources contribute (recycled) material to the archaeological soils in the Negev, too, but these might be difficult to identify due to their similarity to remote dust.

Climatic effects such as temporary higher wind speeds and denser vegetation cover could be reflected by the grain sizes of hilltop ruin soils at Horvat Haluqim. Lucke et al. [19] tentatively concluded from the presence of higher fractions of fine sand in Late Bronze-Early Iron Age layers of hilltop ruins that these might have been associated with more frequent cyclones passing the Negev, leading to more frequent strong winds, higher precipitation, and denser vegetation. Chemically, these layers are characterized by slightly lower $\mathrm{CaCO}_{3}$-contents, possibly due to stronger leaching.

In the Petra region, no comparable evidence can be identified. As well, sand and $\mathrm{CaCO}_{3}$-contents are more problematic there as they are closely connected with contributions from rocks. Puddles of 
temporary water may be responsible for increased clay content, hence clay content is not suitable as indicator of moister climate.

\subsection{Role of Remote Sources}

As local dust sources may include re-mobilized older dust, they pose a challenge for the evaluation of distant sources. Some studies focused, therefore, on suspended dust in the atmosphere, assuming it comprises mainly distant sources. Studies of suspended dust collected in Jerusalem [80] and the Dead Sea [81] demonstrated the same mineralogical and chemical composition of dust particles of several synoptic conditions that carry desert dust. The single variable parameters were somewhat higher palygorskite and $\mathrm{Mg}$ amounts in dust that originated from Arabian deserts.

However, it is questionable whether the composition of suspended dust matters with regard to dust deposition, as the composition of settled dust collected in the Dead Sea region [82] was found to differ significantly from suspended dust in the area [83]. In this context, Yaalon and Ganor [84] suggested that only $1-7 \%$ of the dust transported over Israel settles, mainly discontinuously during pulses of storms superimposing a rather uniform background dust load [85,86]. The dust samples collected during our study largely match the variable compositions reported by other authors $[76,77,87,88]$. An important aspect of settling dust is the presence of silt grains coated by iron and clay, which may have formed before aeolian transport, but could also resemble a 'harvesting' of suspended dust by larger grains during storms, especially when associated with high moisture $[89,90]$. Such coatings could be connected with the formation of aggregates during transport [91,92] and match the observed association of calcareous silt and enrichment of elements and extractable iron in the archaeological soils.

In Jordan, dust settling during five major Khamaseen dust storms showed considerable chemical variability, but no spatial trends [93]. It is likely that such dust is fed from various sources that mix during long-distance transport. As in Israel, coatings rich in Fe and other major and trace elements were observed on silt grains. The Khamaseen dust storms showed Ti/Nb-ratios of 236-340, which were interpreted to indicate similar provenance of siliclastic sources, while $\mathrm{Rb} / \mathrm{K}$-ratio of $31-41$ and $\mathrm{Ga} / \mathrm{Al}$-ratios of 2.3-2.8 were assumed to represent rather homogeneous alumosilicates [93]. These results are plotted against our samples in Table 13, although collection from rooftops in urban areas and analysis of only the silt and clay fractions of the Khamaseen storms limit comparability.

Table 13. Average ratios of $\mathrm{Ti} / \mathrm{Nb}, \mathrm{Rb} / \mathrm{K}$, and $\mathrm{Ga} / \mathrm{Al}$ as reported by Abed et al. [93] for dust settled during Khamaseen storms in Jordan, compared with samples of archaeological soils (excluding the monastery garden), the available silt\&clay fractions of archaeological soils in the Petra region, current dust samples, and sandstones in the Petra region (excluding the sandstone weathering crust on Jabal Farasha).

\begin{tabular}{cccc}
\hline Sample Name & Ti/Nb & $\mathbf{R b} / \mathbf{K}$ & $\mathbf{G a} / \mathbf{A l}$ \\
\hline Petra Region: Jabal Haroun & & & \\
Current dust & 324 & 36 & 3.3 \\
Archaeological soils (excluding monastery garden) & 262 & 37 & 2.4 \\
Silt\&clay fractions archaeological soils & 202 & 38 & 2.0 \\
Sandstones & 219 & 49 & 3.8 \\
Khamaseen dust in Jordan reported by Abed et al. [93] & 303 & 36 & 2.6 \\
\hline Negev: Horvat Haluqim & & & \\
Archaeological soils & 298 & 31 & 2.1 \\
Current dust & 318 & 28 & 2.1 \\
\hline
\end{tabular}

$\mathrm{Ti} / \mathrm{Nb}$-ratios of archaeological soils and current dust storms in the Negev move in the same range as Khamaseen storms in Jordan, but $\mathrm{Rb} / \mathrm{K}$ and $\mathrm{Ga} / \mathrm{Al}$ ratios are smaller, which indicates some differences with regard to clay chemistry. Samples from the Petra region are even more different, in particular the silt\&clay fractions of the archaeological soils. They are characterized by significantly lower Ti/Nb-ratios, caused by high $\mathrm{Nb}$-contents which are probably connected with the presence of acid intra-plate magmas [94] as in the Precambrian basement rocks exposed near Petra [39]. This 
suggests either a direct contribution from the Aqaba and Araba complexes, or an indirect one from sediments stored in the Petra sandstones.

It seems, therefore, that even the regional pattern of dust transport and deposition is closely connected with local sources. Dust settling during single storms can be highly variable, depending on wind speed, direction, and the changing erodibility of surfaces. This makes it difficult to compare single storms with soils that formed during long-term accretion of dust. However, the uniformity of the archaeological soils suggest that time and depositional processes homogenized aeolian sediments, making them archives of both dust sources and sedimentation processes.

\section{Conclusions}

The current study continued and extended a previous investigation on hilltop archeological soils in the Northern Negev and Petra region [19], now including sediments in remains of ancient terraces and a cistern cleanout spoil. Apart from some structural differences, mainly connected with the presence of V-horizons and crust and clast covers at the hilltop ruins, the various ruin types were characterized by very similar substrates. Grain size compositions suggest that aeolian processes are decisive for the composition and primary deposition of sediments at all studied soils including the terraces. Fluvial processes such as runoff irrigation re-distribute aeolian material and play a role for the speed of sediment aggradation, but do not affect the physical and chemical composition of the substrates. Puddles of temporary standing water in cisterns and behind terrace walls may cause higher conductivities and clay contents, resembling the effect of wet dust collectors.

The chemical composition of archeological soils of all types is uniform in the Negev, but variable in the Petra region, due to contributions from local rocks that also increased sedimentation rates. Sandstones contribute high amounts of sediment of medium sand size, mainly by aeolian transport over short distances of less than $100 \mathrm{~m}$. Contributions from dolomitic limestone were evident by elevated concentrations of $\mathrm{CaO}$ and $\mathrm{MgO}$ in the associated soil profiles, independent of grain size. In contrast, rocks in the Negev play a negligible role due to rock hardness, and probably also due to surfaces sealed by biological crust, which appear more scattered in the Petra region.

Precipitation led to a higher presence of silt-rich material in current dust samples in both study areas, associated with elevated contents of major and trace elements, probably due to iron-rich coatings of silt grains. In-situ formation of pedogenic iron seems negligible. This includes Pleistocene paleosols in the Negev which show geochemical indices suggesting minimally stronger chemical weathering than in Holocene soils, mainly due to leaching of $\mathrm{CaCO}_{3}$ connected with the formation of secondary carbonate nodules. A rather homogeneous aeolian source of extractable iron seems present in the Negev, while in the Petra region it is partly inherited from the sandstones.

Remains of Pleistocene paleosols were found buried by various archaeological ruins in the Negev, suggesting that archaeological soils contain varying contributions from now eroded, but possibly partly recycled paleosols. The role of local sources in the Negev is difficult to identify due to similarity of older and recent dust. Nevertheless, proximity effects of local dust sources in the Negev are indicated by elevated levels of $\mathrm{P}_{2} \mathrm{O}_{5}$ in a hilltop ruin which apparently marks an aeolian contribution from remains of a surrounding settlement. The similarity of substrates in the various types of archaeological ruins, the small variations of in-situ weathering indicators, and the apparently subordinate role of fluvial deposition processes suggest that repeated mixing and re-deposition of similar aeolian materials from local and remote sources takes place in the Negev.

Comparison of the archaeological soils with settled dust sampled during Khamaseen storms in various cities in Jordan suggests that local, regional, and remote sources play a role for the silt and clay fractions of the archaeological soils. However, mixing with distance, and the variability of single dust storms, obscures the contribution of individual sources. The archaeological soils of this study suggest that settling aeolian sediments do not directly mirror dust composition in the atmosphere, but also processes of transport and deposition. Archaeologial ruins are very effective Holocene dust traps, probably because of the combined effect of rough surface, wall remains that slow down winds, and 
presence of clast covers, crusts, and some vegetation which play a role for the fixation of dust. This could explain why no similar aeolian deposits were found in natural soils.

Comparison with the studied Pleistocene paleosols suggests that dust sources did not change significantly. However, more frequent snowfall during the Ice Ages could have enhanced settling of dust from the air, fostered formation of biological crusts, and reduced runoff while water infiltration into the soil would have been optimal. The disappearance of snow could be the main difference explaining the absence of significant loess deposition during the Holocene.

Author Contributions: Conceptualization, B.L., A.S. and H.J.B.; methodology, B.L., A.S., K.A.V., N.P., and R.B.; software, K.A.V.; validation, B.L., A.S., K.A.V., N.P., H.J.B., N.A.-J. and R.B.; formal analysis, B.L., A.S., and K.A.V.; investigation, B.L., A.S., H.J.B., N.A.-J., N.P., R.B., and P.K.; resources, B.L., A.S., and R.B.; data curation, B.L.; writing-original draft preparation, B.L. and A.S.; writing-review and editing, B.L., A.S., K.A.V., N.P., H.J.B., N.A.-J., and P.K.; visualization, B.L.; supervision, B.L., A.S., H.J.B., N.A.-J. and R.B.; project administration, B.L.; funding acquisition, B.L., H.J.B., and N.A.-J.

Funding: This research was funded by the German Research Foundation (DFG), grant number LU 1552/2-1.

Acknowledgments: This work was made possible by a grant of the German Research Foundation (DFG, grant no. LU 1552/2-1), which we gratefully acknowledge. We would like to thank the Department of Antiquities of Jordan and the Israel Antiquities Authority for their support. As well, we are grateful to Paula Reimer for ${ }^{14} \mathrm{C}$-dating charcoal from the monastery garden. We are indebted to Ulrich Hübner and the Natural History Society of Nuremberg for their support with sampling Umm Saysaban, and to Moritz Kinzel and the Shkarat Msaeid excavation team. We would like to thank Saleh Suleiman and Ghassem Mohammed for their indispensable help with collecting dust samples on Jabal Haroun. Last but not least, we are grateful to two unknown reviewers and the editor Jan Perlwitz whose constructive comments helped to improve the manuscript.

Conflicts of Interest: The authors declare no conflict of interest. The funders had no role in the design of the study; in the collection, analyses, or interpretation of data; in the writing of the manuscript, or in the decision to publish the results.

\section{Appendix A. Detailed Description of Sampled Sites}

\section{Appendix A.1. Sampled Profiles in the Petra Region}

The investigation region in Southern Jordan is located at Jabal Haroun, a mountain of religious significance where the brother of Biblical prophet Moses, Aaron/Haroun, is supposed to be buried. During antiquity, a large, flat plateau of the mountain was used for worship by a Nabatean pagan sanctuary that was later built over by a Christian monastery. In addition, a chapel was built on the summit of the mountain which hosts today an Islamic weli with a cenotaph that can be visited by pilgrims and tourists [95].

Appendix A.1.1. Natural Sandstone Plateau Soil (Samples Sandplateau)

Rather flat sandstone plateaus in the study area near Petra are sometimes covered by fines if vegetation, in particular trees, is present. A $70 \mathrm{~cm}$ deep profile was excavated next to a large Pistacia atlantica and some Juniperus trees (N 30.41564, E 35.46117) (Figure A1A). Some bushes $<30 \mathrm{~cm}$ and grass patches were present, too. There was no reaction with $\mathrm{HCl}$. The soil was classified as Protic Arenosol (Ochric) according to WRB [52] (see Lucke et al., 2019, Appendix A.2.6, p. 38). 


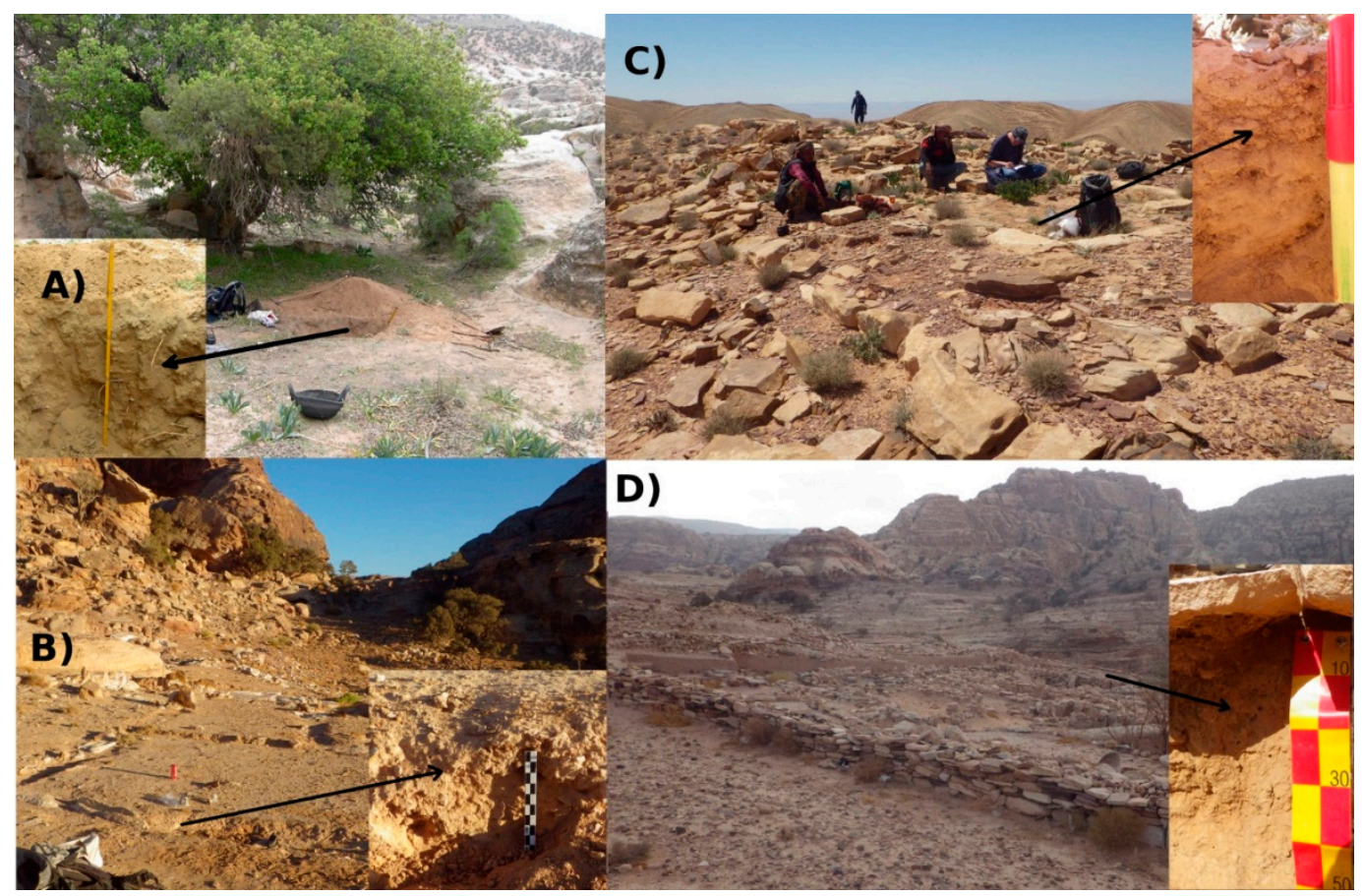

Figure A1. Profiles sampled in the Petra region in Southern Jordan. (A) Natural hilltop sandstone soil. Note the large Pistacia atlantica tree in the background and some Juniperus trees to the right. (B) Soil in wall remains on the site of Umm Saysaban, and (C) soil in the triclinium of Jabal Farasha. Note the clast-covered surface. (D) A paleosol below the partly excavated site of Shkarat Msaeid. The tape measure is in $\mathrm{cm}$.

\section{Appendix A.1.2. The triclinium on Jabal Farasha}

The summit of Jabal Farasha, south-west of Jabal Haroun, was used for cultic purposes with a triclinium from the 1st century BCE till the 2nd century CE [96]. The open space between the triclina consisted of cleared, smoothed sandstone rock. It was covered by about $25 \mathrm{~cm}$ of wind-blown sediment (Figure A1C). There are no archaeological sediments derived from mudbricks, walls, or other debris, whereas the topography does not enable fluvial deposition. The triclina walls had not yet completely filled with sediments, and the soil surface was covered by small clasts and a crust. Occasional roots from small bushes and patches of grass were present. The sandstone at the bottom of the profile appeared weathered, broken into small plates with some fine material in-between small cracks. Reaction to $\mathrm{HCl}$ was strong in the soil, but nil in the rock. The soil classified as Calcaric Leptosol (Protic) according to WRB [52]. Three soil samples and one of the underlying rock were collected ( $\mathrm{N}$ 30.30445, E 35.40141) (see Lucke et al. [19], Appendix A.2.2, p. 36).

\section{Appendix A.1.3. Umm Saysaban Hilltop Ruin}

The Bronze Age site of Umm Saysaban was constructed on a high, but relatively flat, sandstone plateau north-west of Jabal Haroun. Excavations revealed that ancient buildings were constructed on the smoothed sandstone bedrock, and a layer of wind-blown sediment accumulated after the desertion of the site. Fluvial deposition appears unlikely, and there were no indications of remains of archaeological sediments derived from mudbrick, mortar made from mud, or other debris. The soil surface was covered by a crust and many small, subangular sandstone fragments. Occasional roots from small bushes $<30 \mathrm{~cm}$ and patches or grass were present throughout the profile. Two samples were taken from a fresh exposure (N 30.34595, E 35.43178, Figure A1B). Reaction to $\mathrm{HCl}$ was strong. The profile of Umm Saysaban was classified as Calcaric Leptosol (Protic) according to WRB [52].

For further description, see Lucke et al. (2019, Appendix A.2.3, pp. 36-37). 
Appendix A.1.4. Early Holocene Paleosols of Shkarat Msaied and Abu Suwwan

The Pre-Pottery Neolithic B (PPNB) hilltop site of Shkarat Msaeid (N 30.44372, E 35.43917) was dated to 9200-7700 BCE [97]. A paleosol profile of $\sim 60 \mathrm{~cm}$ (Figure A1D) had been buried below the Neolithic walls. Secondary calcium carbonate nodules, calcified root channels, and large pores were present, and the paleosol was classified as Cambic Calcisol (Hypocalcic) according to WRB [52]. A bright horizon on top of the sandstone, representing a BCk-horizon possibly partly derived from aeolian deposits, was analyzed (sample Shkarat Msaied 1).

The unexcavated Pre-Pottery Neolithic B (PPNB) site Abu Suwwan is located on a hilltop of Jabal al-Bara (N 30.33064, E 35.42297, Figure A2A). A paleosol was preserved below a Neolithic wall, representing a truncated 3BC-horizon (sample Abu Suwwan below nw $65 \mathrm{~cm}$ ), apparently containing ash from the settlement. Reaction to $\mathrm{HCl}$ was strong. It was classified as Cambisol (Protocalcic) according to WRB [52].

For further description of the two sites, see Lucke et al. [19], Appendix A.2.4-A.2.5, pp. 37-38).

\section{Appendix A.1.5. The Monastery Garden of Jabal Haroun}

Remains of walls form a rectangular structure to the southeast of the ruins of the monastery on Jabal Haroun (Figure A2B). This area had been suspected to represent a former garden, possibly irrigated with water from cisterns (site 2, area E in Silvonen et al. [98], p. 347). Fluvial deposition is unlikely here since the garden is near the top of a slightly inclined sandstone plateau, so that the outer wall prevented or greatly limited it. The maximum runoff catchment area not considering the wall is $0.007 \mathrm{~km}^{2}$, or $0.002 \mathrm{~km}^{2}$, assuming the wall limited runoff. However, it seems likely that the surrounding, weathered sandstones contributed aeolian sediments. Remains of smaller walls were detected inside the surrounding rectangular wall. We excavated a profile next to one of the smaller walls near the center of the rectangular area (N 30.31665, E 35.40518).

Sandstone bedrock was reached in $70 \mathrm{~cm}$ depth. There was no or only a weak reaction to $\mathrm{HCl}$ throughout the profile which was sand-dominated. The upper $35 \mathrm{~cm}$ had rose color. At $35-60 \mathrm{~cm}$, color was grayish-red and charcoal and bone fragments were present, which were interpreted as remains of ash added as manure. A few Late Byzantine and Early Islamic pottery sherds were found at the surface, while two Early Byzantine pottery pieces were encountered at a depth of $40 \mathrm{~cm}$. At the bottom, at $70 \mathrm{~cm}$ depth, the soil was reddish and quite compact, and contained some Late Roman pottery. A charcoal piece from a depth of $50 \mathrm{~cm}$ was submitted to radiocarbon dating, and an OSL-sample was taken from a depth of $60 \mathrm{~cm}$. Occasional small stones were present in the profile, and many roots were present in the upper $30 \mathrm{~m}$. Patches of grass and various bushes grew on the surface, which was nearly completely covered by stones of variable size. Silvonen [99] (p. 120) found in 1998 a surface pottery density of more than 0.04 pieces $/ \mathrm{m}^{2}$ in the rectangular area behind the monastery. Our re-survey of the pottery cover in 2016 found 0.24 pieces $/ \mathrm{m}^{2}$. Preliminary dating of the surface sherds suggests a dominance of Byzantine-Ummayyad (transitional?) material, with a small Nabatean component and probably some Late Islamic sherds. The soil is classified as Protic Arenosol (Alcalic, Ochric) according to WRB [52]. 


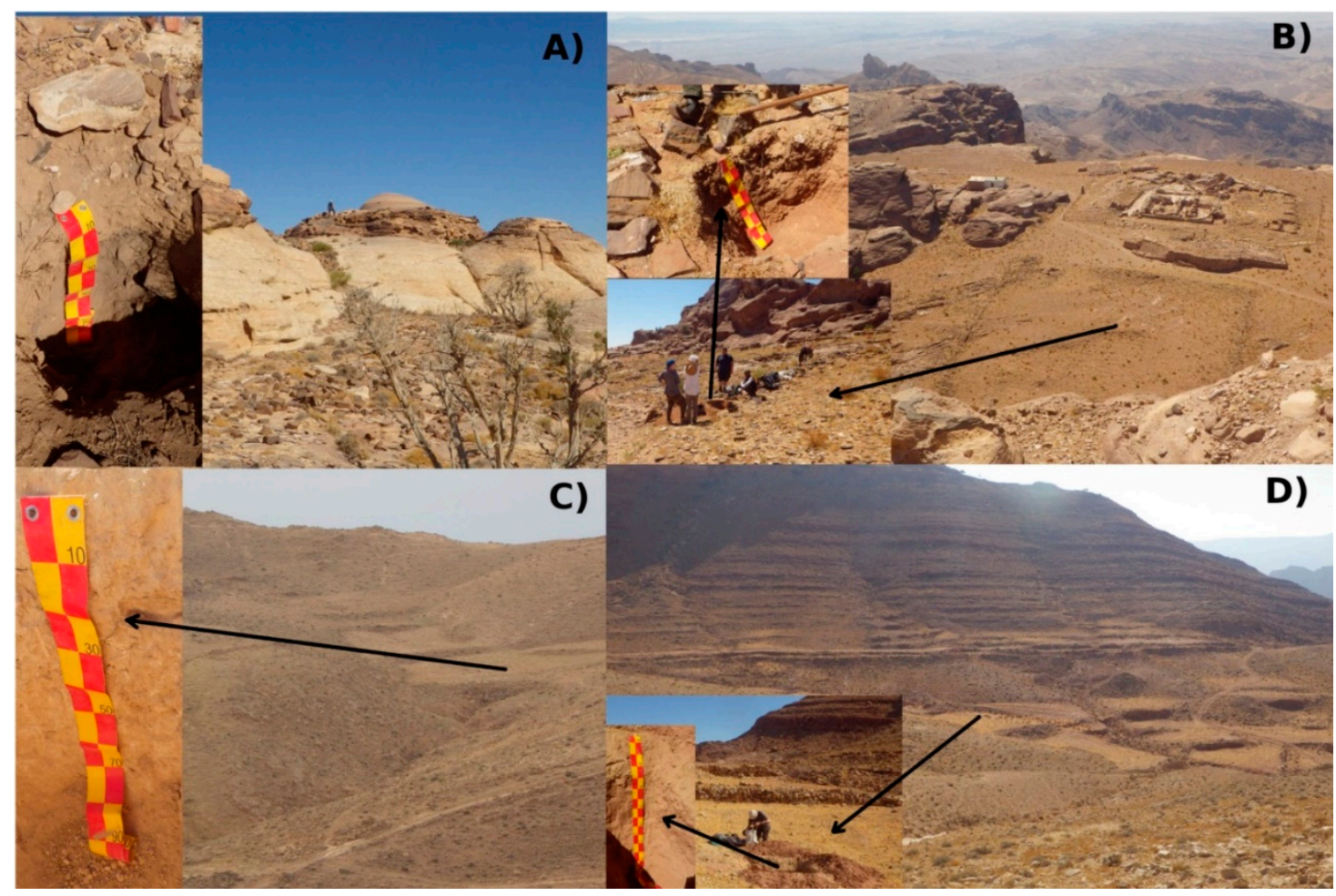

Figure A2. Profiles sampled in the Petra region in Southern Jordan. (A) A paleosol below the unexcavated pre-pottery Neolithic site of Abu Suwwan (the site is the stone scatter in front). (B) A profile in the probable monastery garden on Jabal Haroun. Note the rectangular wall remains below the ruin of the monastery, which apparently surrounded the garden. The stone pile between the monastery ruin and the rectangular enclosure is excavation debris. (C) A profile on the runoff-collecting terraces on dolomitic limestone, and (D) a profile on the runoff-collecting terraces on sandstone. Note that the parallel lines on the steep sandstone slope in the background partly represent geological layers, and partly remains of ancient terraces. The tape measure is in $\mathrm{cm}$.

\section{Appendix A.1.6. The Terrace on Dolomitic Limestone (Jh Site 60)}

A dolomitic Turonian limestone (Wadi As Sir formation) [39] forms a ridge located west of Jabal Haroun. Small terraces cross the depressions of developing wadis from the very top of the ridge until approximately the middle of the slope. This design suggests that water was slowed down by the terraces from the beginning of runoff formation, allowing water and sediments to be harvested behind terrace walls. Today, the terraces are completely filled with soil, and probably stopped collecting further sediment. Local Bedouins occasionally plow the terraced areas in order to grow cereals. This location is part of area K (site 60) described by Lavento et al. [100] (pp. 220-222) and Silvonen et al. [98] (p. 366). The terrace's catchment was estimated to $0.044 \mathrm{~km}^{2}$.

A profile was excavated in the center of the terraced area (N 30.31244, E 35.39476) (Figure A2C) down to bedrock and a layer rich in angular debris at $\sim 70 \mathrm{~cm}$ depth, which probably represents a former, debris-covered rocky slope. Occasional small dolomitic limestone debris was present throughout the profile. Small roots were present in the upper $20 \mathrm{~cm}$. No layers or artifacts could be observed, and the texture appeared silt-dominated throughout, with strong reaction to $\mathrm{HCl}$. The upper $10 \mathrm{~cm}$ were friable probably due to recent plowing. The soil became increasingly compact with depth. The surface was covered by occasional stones, patches of grass, and scattered, small bushes. Two OSL-samples were taken in 20 and $50 \mathrm{~cm}$ depth. The Finnish Jabal Haroun Project (FJHP) recovered $\leq 0.01$ pottery pieces $/ \mathrm{m}^{2}$ [99]. Our re-survey in 2016 found 0.06 pottery pieces $/ \mathrm{m}^{2}$, all dating to the $1 \mathrm{st}-2$ nd century CE. The soil is classified as Protic Calcaric Regosol (Colluvic) according to WRB [52]. 
Appendix A.1.7. A Terrace on Sandstone (Jh Site 33)

The Wadi As-Saddat emerges between the above mentioned dolomitic limestone ridge and the southern slope of Jabal Haroun, and hosts one of the largest runoff cultivation systems of the mountain (site 33) [100] (Figure A2D). Many of the barrages are double-walled, multi-phased, and sturdy. In Sector $\mathrm{D}$, in the approximate center of the area, a stepped structure had been excavated that was interpreted as spillway or sluice gate to control the flow of water [100]. We excavated a profile of $150 \mathrm{~cm}$ in a terrace in the center of sector C (N 30.31404, E 35.39839). Its catchment is estimated to $0.095 \mathrm{~km}^{2}$.

At the bottom of the profile, much angular rock debris were present, probably of an old surface with a debris layer on the bedrock. Silt-dominated sediment followed from 140 to $70 \mathrm{~cm}$ depth. Soil structure was compact and platy, with few stones, and some roots. At $20-70 \mathrm{~cm}$, the soil was much less compact, dominated by brown sand, and stone content increased slightly. The upper $20 \mathrm{~cm}$ had been plowed not long ago, was sand-dominated, and had high stone content. The top layer and the silt-dominated layer showed strong reactions to $\mathrm{HCl}$, while the sandy layer showed a weaker reaction. The sandstone terrace profile could receive fluvial deposition from the sandstone slope it was built on, which resembles the largest area of its catchment, but also from a smaller catchment on the adjacent Wadi As Sir dolomitic limestone. The Finnish Jabal Haroun Project (FJHP) recovered a pottery density of $\leq 0.01$ pieces $/ \mathrm{m}^{2}$ [99]. Our re-survey in 2016 found 0.06 pieces $/ \mathrm{m}^{2}$, mainly Nabatean from the 1st-2nd century CE, and few Late Roman, Byzantine, Early Islamic, and Late Islamic sherds. The soil is classified as Protic Calcaric Arenosol (Colluvic) over Protic Calcaric Regosol (Colluvic).

\section{Appendix A.1.8. Petra Region Reference Samples}

Reference sites were sampled in order to tackle the potential contributions of local sources to the studied sediments. Two active sediment fans were sampled near the villages of Beidha (N 30.37694, E 35.45327) and Umm Sayhoun (N 30.34793, E 35.45814). Sandstone samples were collected from outcrops of the prevailing geological formations in the Petra area: Abu Khushayba (sample JH, N 30.31734, E 35.40418), Umm Ishrin (N 30.32192, E 35.43099), and Disi sandstones (N 30.34763, E 35.45844 and sample Ba'ja sandstone, N 30.41564, E 35.46117). One sample was collected from an outcrop of the Turonian Wadi as Sir dolomitic limestone (N 30.31261, E 35.39468).

\section{Appendix A.2. Sampled Profiles in the Negev}

We sampled sediments in archaeological structures near the site of Horvat Haluqim. The main remains of the site is a large oval structure, interpreted by Cohen [101] as Iron Age fortress with casemate walls. Three small adjacent wadis discharge into the main wadi Nahal HaRo'a, at the crossroads of important routes. The site includes preserved ancient, runoff-collecting terraces, cisterns with conduit cannels collecting water from the slopes, a Roman watchtower, a hilltop cairn, and the ruins of some other round and rectangular buildings. Most of the slopes are devoid of soil cover and the hard Turonian limestone is exposed. All samples collected in the Negev were silt-dominated and showed a strong reaction with $\mathrm{HCl}$.

\section{Appendix A.2.1. Terrace on Chalk in a Tributary of Nahal HaRo'a}

Some $3 \mathrm{~km}$ east of Horvat Haluqim and not associated with an ancient settlement, runoff collecting terraces were preserved on soft chalk phosphorite of the Mishash Formation and chalk of the Menuha formation. Most terrace walls were constructed using large local chert stones. The terrace walls are filled with fine sediments and some gravel layers. At the base of the slopes of the valley, there are older colluvial aprons of (re-deposited) loessial sediments. These are mostly covered by chert debris of varying sizes. Some of the chert-covered old aprons might represent "hogbacks", i.e., rests of ancient surfaces once extending from the hills to the valleys that were later cut by erosion. The soils of the 
loessial aprons show secondary carbonates and cambic horizons and probably represent Pleistocene paleosols [8].

The distribution of chert on the current soil surface is irregular, and it seems possible that human action contributed to the pattern. The colluvia at the lowermost valley slopes show a limited cover of chert, probably due to removing stones before plowing.

We studied a terrace in the lower part of the valley (N 30.90111, E 34.84350, see Figure A3A, profile to the left). Its catchment size was estimated to $0.41 \mathrm{~km}^{2}$. Both sides of the wall remains were excavated and showed identical stratigraphy. The uppermost $15 \mathrm{~cm}$ (horizons A and C, sample Haroa Farm $10 \mathrm{C}$ ), with occasional angular chert debris seem to represent colluvial sedimentation from the bordering slope. Remains of numerous small crusts probably formed after small sedimentation events, suggesting that the terrace was not plowed anymore. Various shrubs of 30-50 cm height grew in the vicinity and suggest that the terrace sediments still store significant amounts of water.

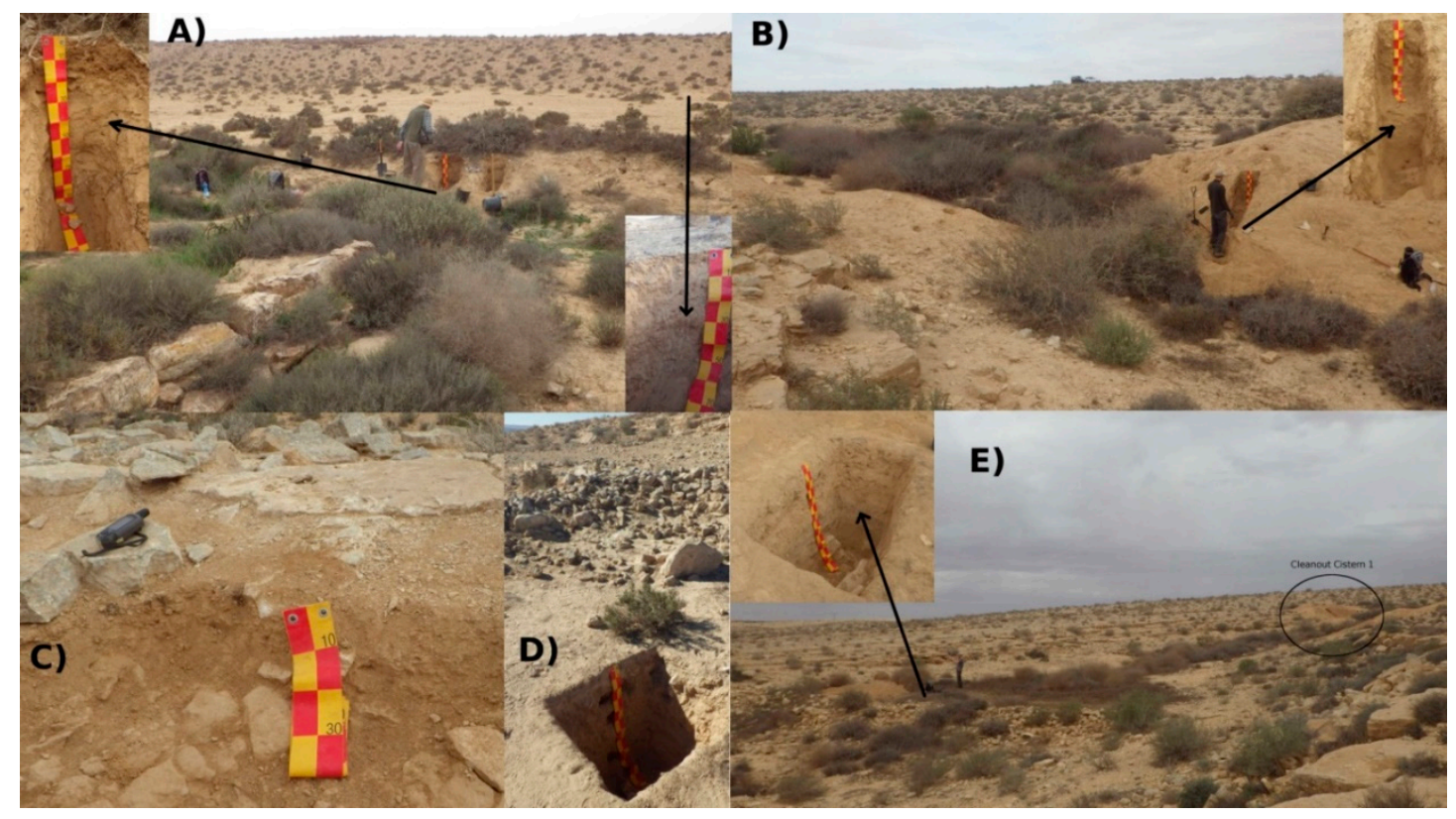

Figure A3. Profiles sampled in the Northern Negev in Israel. (A) A profile of the runoff-collecting terrace in a tributary of Nahal HaRo'a (left), and a loessial paleosol in the nearby colluvial-aeolian apron (right). (B) A profile excavated in the sediment pile cleaned out from Cistern 1 in the western wadi of Horvat Haluqim (to the left-vegetation obscures the structure, but marks the area where water is currently gathering). (C) A soil in the hilltop tumulus grave structure at the western wadi of Horvat Haluqim. (D) A profile in the hilltop ruin at the central wadi of Horvat Haluqim. (E) A profile excavated in Terrace 3 at the western wadi. Vegetation shows where moisture is still gathering today. Note the sediment cleanout pile of Cistern 1 to the right: water was first channeled into open cisterns in the uppermost part of the valleys, and when these were filled, it ran to the fields below. The tape measure is in $\mathrm{cm}$.

From 15 to $28 \mathrm{~cm}$, alluvial sediment was present, dominated by small, sorted gravel with banded bedding structure, interspersed with silt (2C-horizon). This suggests that the terrace walls filled up when these sediments were laid down, and occasional floods moved gravel to the sides. The terrace walls were apparently still intact, but not used any more, since plowing should have destroyed the bedding structure. A darker layer at $28 \mathrm{~cm}$ was interpreted as buried Ah-horizon probably marking an old, cultivated surface (Horizon 3Apb, sample Haroa Farm 28 fAh). Sediments at 30-55 cm apparently reflect cultivation (Horizons $3 \mathrm{C} 1$ and $C 2$, samples Haroa Farm $40 \mathrm{C}$ and $50 \mathrm{C}$ ). They were very homogeneous and showed no bedding structure, suggesting plowing, and were more compact. At 55-70 cm, gravelly alluvium may represent pre-cultivation sediment (Horizon 4C, sample Haroa 
Farm 65 C). At 70-90+ cm depth, the very bottom of the profile, a paleosol, free of stones and with pronounced, large secondary carbonate nodules, appeared (5Bk-horizon, sample Haroa Farm 80 B). It was probably truncated by the lower alluvial layer. The paleosol was slightly more compact and brownish than the sediment on top. Occasional small roots of $<1 \mathrm{~mm}$ in diameter were present throughout the profile.

The terrace soil is classified as Protic Calcaric Regosol (Colluvic, Siltic) over Calcaric Fluvisol (Aric, Siltic) over Cambic Calcisol (Siltic) according to WRB [52].

\section{Appendix A.2.2. Nahal HaRo'a Paleosol of Loessial Apron}

A paleosol, characterized by a cambic horizon and secondary carbonate nodules typical for Pleistocene soils in the area, developed in a loessial apron near Nahal HaRo'a. It had been exposed by a gully (N 30.30140, E 34.84296, see Figure A3A, profile to the right). A 1 mm thick crust (biological or physical) covered the current surface, and various bushes $<30 \mathrm{~cm}$, occasional small stones, and patches of grass were present. Occasional small roots $<1 \mathrm{~mm}$ in diameter could be observed throughout the profile. A V-horizon characterized by yellow color (10 YR 8/6) seems present in the upper $25 \mathrm{~cm}$, while brownish-yellow color (10 YR 6/6) and secondary carbonates appeared in $\sim 25 \mathrm{~cm}$ depth and below, suggesting a layer change and the presence of a buried, probably truncated, 2Bwk horizon. The presence of a few small subangular stones at the layer change supports truncation: as stones are otherwise mostly absent from the profile, these probably mark an old land surface. According to WRB [52], the soil developed in the upper $25 \mathrm{~cm}$ is classified as Protic Calcaric Regosol (Siltic), and the buried paleosol as Cambic Calcisol (Siltic). For further description, see Lucke et al. [19] (Appendix A.1.3, pp. 34-35).

Appendix A.2.3. The Cistern Cleanout in the Western Wadi

In the upper part of the western wadi of Horvat Haluqim, the ancient irrigation system was designed to first collect runoff water from the rocky slopes in a cistern (numbered Cistern 1, see Figure A3B). The catchment of the cistern was estimated to $0.043 \mathrm{~km}^{2}$. When it was full, water would flow to the terraces. This cistern had apparently been cleaned rather recently as it contained little sediment. It still collects water and is covered by dense vegetation. Next to the cistern, two piles of silty cleanout sediment were observed. Junge et al. [59] were able to date a cistern cleanout sediment spoil from the nearby site of Atar Haroa by OSL (in that case to the medieval period). We excavated trenches in both cleanout piles, and decided to focus on the larger one (Cleanout 1), which contained significant amounts of pottery. A preliminary identification suggested that the material dates largely to the Byzantine and Early Islamic periods, and might mainly stem from broken water bottles.

Both cleanout piles were affected by bioturbation of large mammals, probably porcupines, which are today frequent in the area. However, the sections that we dug at the sides of the piles were apparently not affected. The total height of the profile in the first cleanout pile was $160 \mathrm{~cm}$ with a layer change in about $60-80 \mathrm{~cm}$ that was associated with a change of sediment structure from friable to compact. As well, there was a concentration of pottery in that depth, probably marking an old surface that was exposed for a longer time. Broken chunks of sediments could still be observed as they were probably excavated from the cistern. Large aggregates could be observed in the upper layer, and smaller ones in the lower layer. This may represent a gradual loss of structure with time, possibly connected with pedochemical processes. The cistern sediments were free of stones, and the surface of the cleanout piles covered by a thin crust. Vegetation was not present and no A-horizon could be identified. On some sediment aggregates throughout the piles, white calcareous layers could be observed which might represent remains of a plaster cover of the former cistern bottom. On a macroscopic scale, no further structures could be observed. At the very bottom, a buried paleosol with cambic horizon and secondary carbonates appeared, suggesting that some loess soil cover of the today rocky slopes might still have been present during antiquity. Reaction to $\mathrm{HCl}$ was strong throughout 
the cleanout profile. OSL samples were taken from the upper and lower border of the compact, lower cleanout layer in 110 and $150 \mathrm{~cm}$ depth.

Interviews of Bedouins and local inhabitants who are still using cisterns in Jordan suggest that such open water basins were not made to store water for a long period, but that it was soon collected in jars, bottles, or skins in order to prevent evaporation. Alternatively, easily accessible water basins might have been used to water grazing animals during the wintertime. Currently used cisterns in southern Jordan, at least if intended for human use, are regularly cleaned of sediment in time spans of a few years in order to prevent or reduce the development of parasites. Since Cistern 1 is relatively difficult accessible from the slopes and associated with pottery of water bottles, it seems likely that the collected water was destined for human use. Therefore, the OSL-ages of the sediments likely refer to the time of deposition during cistern operation, since the time gap till regular cleaning should not have exceeded the error of the measurement.

The cistern cleanout pile is classified as Protic Calcaric Regosol (Siltic, Transportic) over Calcaric Regosol (Siltic, Transportic) over Camic Calcisol (Siltic) according to WRB [52].

\section{Appendix A.2.4. Western Wadi Hilltop Ruin (Samples HH-WW R1)}

A Bronze Age tumulus grave that was not yet excavated is located at a summit of a slope of the western wadi of Horvat Haluqim (N 30.89151, E 34.79909, Figure A3C). The cairn was possibly already looted and destroyed during antiquity and had largely collapsed, but $20 \mathrm{~cm}$ of sediment fill accumulated in a wall that once surrounding the tumulus. The soil was clearly of aeolian origin and classified as Calcaric Leptosol (Protic, Siltic), according to WRB [52].

A more detailed description can be found in Lucke et al. [19] (Appendix A.1.1, p. 33).

\section{Appendix A.2.5. Central Wadi Hilltop Ruin (Samples HH-CW Ruin)}

A circular structure on a small loess apron projecting into the central wadi of Horvat Haluqium was studied (N 30.88948, E 34.80015, Figure A3D). At least $60 \mathrm{~cm}$ of aeolian sediment accumulated within the structure. It was constructed above a loessial paleosol containing secondary carbonate nodules. Similar to the cistern cleanout pile, this suggests that in antiquity loess soils were still preserved to a larger degree than today. OSL samples were taken from 25, 55, and $90 \mathrm{~cm}$ depths. This ruin soil was classified as Protic Calcaric Regosol (Siltic) over Cambic Calcisol (Siltic) according to WRB [52] (see Lucke et al. [19], Appendix A.1.2, pp. 33-34).

\section{Appendix A.2.6. Western Wadi Terrace 3}

Downslope from Cistern 1, in the upper part of the western wadi of Horvat Haluqim, terraces begin to appear in rather regular intervals. We excavated the third terrace which had been constructed with a massive, well-preserved wall (Figure A3E). Its catchment was estimated to $0.053 \mathrm{~km}^{2}$. The upper $\sim 60 \mathrm{~cm}$ of the sediments were strongly bioturbated as shown by remains of filled animal burrows (Krotowinas). This part of the profile was characterized by small, banded layers resembling fluvial sorting and/or buried surface crusts, which probably formed when the terrace was not plowed. Compact silty sediment without bedding structure occurred at $\sim 60-120 \mathrm{~cm}$ depth. At $120 \mathrm{~cm}$, several large limestones and bedrock appeared. It seems possible that the large stones represent the remains of an older, small terrace wall that was buried by sediments and not built higher as the terrace wall might have been reconstructed further downslope. Between these limestones, at the very bottom of the profile, thin remains of a (probably Pleistocene) paleosol with weakly developed secondary carbonate nodules appeared.

At $\sim 90-100 \mathrm{~cm}$ depth, a grayish-brown layer with some occasional stones was present, interpreted as remains of ashy refuse perhaps deposited for manuring purposes. A few soft, small white "nodules" of material rich in calcium carbonate were dispersed over the profile. As no veins or traces of secondary carbonate could be identified in the surrounding sediment, we assume that these represent chalk 
or marl transferred from the bordering slopes where some dolomitic marl and palygorskite shale associated with a Turonian paleosol [51] are exposed.

The soil of Terrace 3 is classified as Protic Colluvic Calcaric Regosol (Siltic) according to WRB [52].

Appendix A.2.7. Negev Reference Samples

Reference samples were collected from outcrops: soft limestone (N 30.89068, E 34.79859), hard limestone (N 30.89068, E 34.79859), chalk from the central wadi (N 30.88943, E 34.79994), and chalk from the tributary of Nahal HaRo'a (N 30.89634, E 34.84516). The Turonian paleosol [51] was sampled as well (N 30.88943, E 34.79994). Outcrops were excavated in order to reach an unweathered zone and to make sure that no contamination with surface sediments was possible.

\section{Appendix B. Detailed Methods Description}

\section{Appendix B.1. Sample Collection}

Profiles were excavated until reaching bedrock or rock debris. Soils and substrates were classified according to the WRB [52], while horizons were described according to Soil Survey Staff [53]. The concept of V-horizons as proposed by Turk et al. [102] was applied for vesicular layers. Samples were collected in plastic bags and taken from areas of approximately $5 \mathrm{~cm}$ size in the center of defined layers or horizons in the profiles. Dust samples were collected in plastic boxes filled with standard glass marbles (Figure A4).

Catchment sizes of terraces were estimated using the polygon tool of GoogleEarthPro.

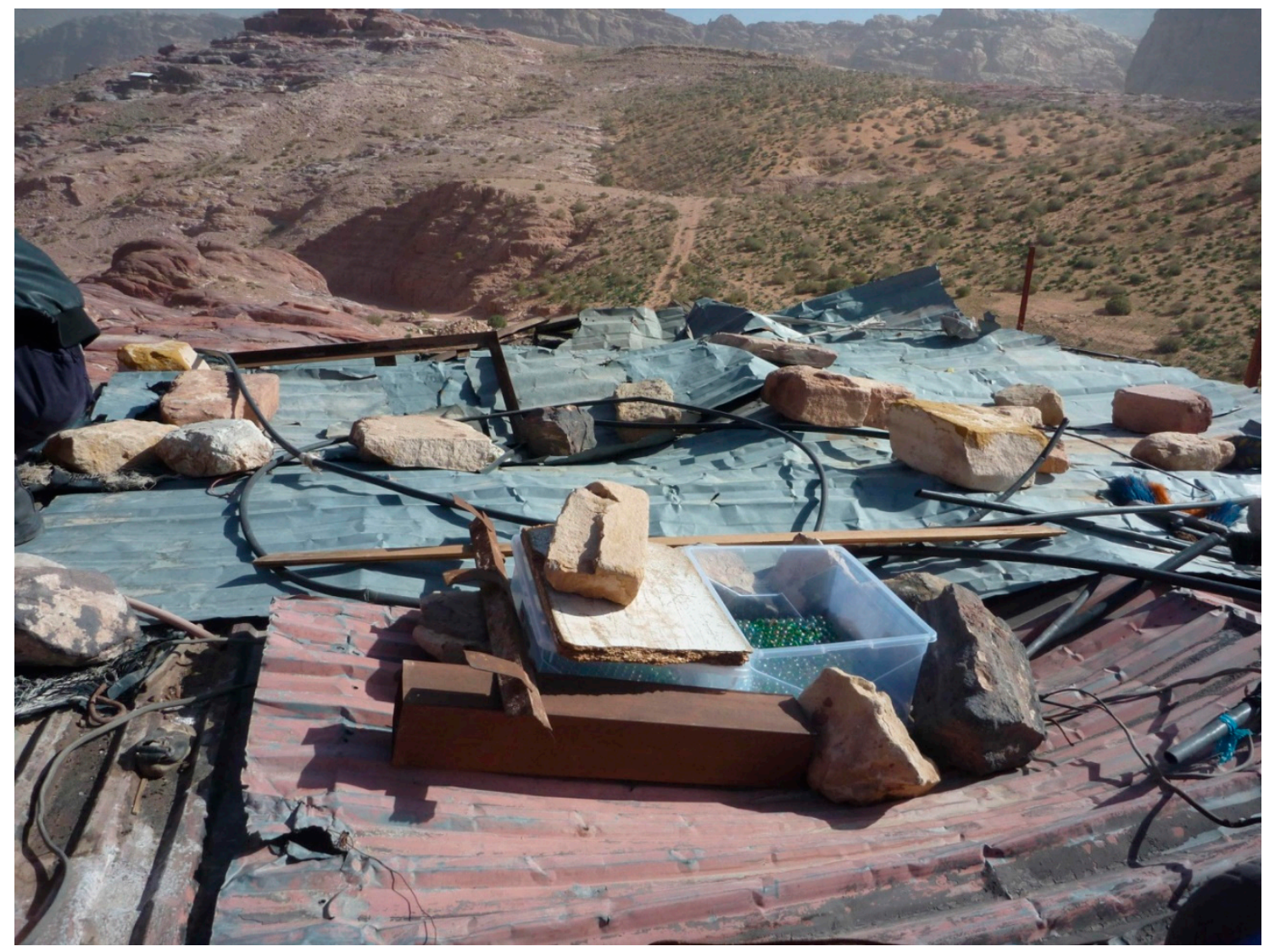

Figure A4. Dust sampler on the roof of Saleh Suleiman's house. Note the ridge of fine sediments in the background to the right, covered by small bushes and patches of grass. From Lucke et al. [19] (p. 10). 


\section{Appendix B.2. Laboratory Analysis}

The samples were air-dried for $72 \mathrm{~h}$ at $40{ }^{\circ} \mathrm{C}$, dry sieved by $2 \mathrm{~mm}$, and all further analyzes conducted with the fraction $<2 \mathrm{~mm}$. An agate ring mill type Retsch RS 200 was used to grind and homogenize samples where pulverized material was needed.

Color was determined by a Munsell Soil Color Chart.

$\mathrm{PH}$ was determined with a glass electrode ( $\mathrm{pH}$-meter 530 by WTW, with electrode InLab 423 by Mettler-Toledo) in a soil: water solution of 1:2.5.

Electrical conductivity was measured with a GMH 3410 conductivity meter by Greisinger electronic in a soil: water solution of 1:5.

Bulk density was determined with $100 \mathrm{~mL}$ cylinders. In order to calculate the bulk density of only the (probably aeolian) fine fraction in hilltop ruins, the volume of the skeleton fraction was determined after sieving by its water displacement.

$\mathrm{CaCO}_{3}$ and organic carbon were determined using an Elementar vario $\mathrm{EL}$ cube $\mathrm{C} / \mathrm{N}$-analyzer in doubles before and after ignition for two hours at $500{ }^{\circ} \mathrm{C}$. The calculation of the $\mathrm{CaCO}_{3}$-content is based on the assumption that ignition removes organic matter and the remaining carbon is bound in $\mathrm{CaCO}_{3}$. For some samples such as rocks, $\mathrm{CaCO}_{3}$-contents were determined using a "Karbonat-Bombe", which applies similar principles as those of the Scheibler-Apparatus [103]. For a comparison of the very similar results from the different methods, see Lucke et al. [19] (Appendix C, pp. 42-43).

Grain size distribution was determined washing samples with distilled water until conductivity fell below $200 \mu \mathrm{S} / \mathrm{cm}$ (without removal of $\mathrm{CaCO}_{3}$ ). Then, sodium hexametaphosphate $\left(\mathrm{Na}_{4} \mathrm{P}_{2} \mathrm{O}_{4}\right)$ was applied for dispersion. Wet sieving determined the sand fractions according to DIN 19,683 [104], while the smaller particles were analyzed with a Sedigraph 5100 (Micromeritics) [105]. As current dust samples were too small to be analyzed by this method, they were measured by a Malvern Mastersizer 3000 using the Hydro-EV dispersion unit with $3000 \mathrm{rpm}$. A quantity of $80 \mathrm{~mL}$ sodium hexametaphosphate $\left(\mathrm{Na}_{4} \mathrm{P}_{2} \mathrm{O}_{4}\right)$ was added and a minimum of 5 min of ultrasonication applied (or until shadowing remained constant). The raw laser diffraction values were transformed into grain size distributions using the Mie scattering model, a refractive index (RI) of 1.54, and adsorption (A) of 0.1. Laser results were mostly calculated as averages of 5 measurements, which showed good reproducibility. For better comparability with Sedigraph results, the clay-silt border of laser measurements was adjusted to $5 \mu \mathrm{m}$ (following Crouvi et al. [11]) and the medium silt fraction mathematically eliminated (a systematic comparison of laser and Sedigraph results is presented by Lucke et al. [19], Appendix E, pp. 50-54).

Some soft rocks were gently crushed in order to simulate physical disintegration, and the grain size distribution measured as described above in order determine an estimate of the particle sizes that could be released from the rocks. For selected samples from the Petra region, silt and clay were collected after the Sedigraph measurement, and washed to remove $\mathrm{Na}_{4} \mathrm{P}_{2} \mathrm{O}_{4}$. Then, the element composition of these silt\&clay fractions was determined as described below.

Major element oxides and selected trace elements were determined by an energy-dispersive X-ray fluorescence spectrometer (XRF) Spectro XEPOS. Precision and accuracy are generally better than $0.9 \%$ for main elements and $5 \%$ for trace elements. The loss of ignition (LOI) was determined by weighing the powdered samples before and after drying: (1) $12 \mathrm{~h}$ at $105^{\circ} \mathrm{C}$ in a cabinet dryer and (2) $12 \mathrm{~h}$ at $1030^{\circ} \mathrm{C}$ in a muffle furnace, melting samples in Pt-crucibles.

Pedogenic iron, aluminium, and manganese oxides were dissolved with sodium dithionite at room temperature according to Holmgren [106]. Weakly crystallized pedogenic oxides were extracted in the dark using buffered ( $\mathrm{pH}$ 3.25) oxalate-solution according to Schwertmann [107]. Their concentrations were measured by ICP-OES (ICAP 6200), and in case of the oxalate extraction, silicium was analyzed as well.

The weight-specific magnetic susceptibility $\chi$ was examined with an Agico MFK1-FA multi-function Kappabridge device using three different frequencies $(976 \mathrm{~Hz}, 3904 \mathrm{~Hz}$ and $15616 \mathrm{~Hz})$ and field strengths between 10 and $700 \mathrm{~A} / \mathrm{m}$. For some samples, an Agico KLY-4S was used with $875 \mathrm{~Hz}$ 
and $300 \mathrm{~A} / \mathrm{m}$. In order to make results between the two devices directly comparable, $424 \mathrm{~A} / \mathrm{m}$ and $976 \mathrm{~Hz}$ were used as reference setting of the MFK1-FA. For those samples in which pedogenic oxides had been extracted, the extracts were dried and measured in the MFK1-FA in order to assess the role of pedogenic iron oxides for magnetic susceptibilities.

\section{Appendix B.3. Dating}

Radiocarbon dating was executed by the ${ }^{14} \mathrm{CHRONO}$ Centre for Climate, the Environment and Chronology, Queen's University Belfast, using a standard acid-alkali-acid (AAA) method. Samples were placed in $100 \mathrm{~mL}$ beakers (cleaned by baking at $\left.450{ }^{\circ} \mathrm{C}\right)$ and immersed in hydrochloric acid $(4 \%$, $30-50 \mathrm{~mL})$. The contents of the beaker were heated on a hotplate $\left(800^{\circ} \mathrm{C}\right.$ for $\left.2-3 \mathrm{~h}\right)$ and the samples then received washes with deionised water until neutral. Sodium hydroxide $\left(2 \%\right.$, at $800{ }^{\circ} \mathrm{C}$ for $\left.1-2 \mathrm{~h}\right)$ was added to remove humic acids followed by rinsing with deionised water until neutral. The acid step was repeated to remove any $\mathrm{CO}_{2}$ absorbed during the $\mathrm{NaOH}$ step, rinsed and dried overnight at $60^{\circ} \mathrm{C}$.

The dried samples were weighed into pre-combusted quartz tubes with an excess of copper oxide $(\mathrm{CuO})$, sealed under vacuum and combusted to carbon dioxide $\left(\mathrm{CO}_{2}\right)$. The $\mathrm{CO}_{2}$ was converted to graphite on an iron catalyst using the zinc reduction method [108]. The graphite was analyzed on a 0.5 MV National Electrostatics compact accelerator mass spectrometer (AMS). The sample ${ }^{14} \mathrm{C} /{ }^{12} \mathrm{C}$ ratio was background corrected using measurements on anthracite and normalised to the HOXII standard (SRM 4990C; National Institute of Standards and Technology). The radiocarbon ages were corrected for isotope fractionation using the AMS measured ${ }^{13} \mathrm{C} /{ }^{12} \mathrm{C}$ ratios which accounts for both natural and machine fractionation. The radiocarbon age and one standard deviation were calculated using the Libby half-life of 5568 years following the methods of Stuiver and Polach [109].

Sediment samples were dated by Optically Stimulated Luminescence (OSL) at the Geological Survey of Israel (GSI). Samples were collected without exposure to sunlight and all laboratory procedures carried out under suitable dim orange-red light. Quartz in the range of 90-125 $\mu \mathrm{m}$ was extracted using routine protocols [20], and the single aliquot regenerative dose (SAR) protocol [110] used to measure the equivalent dose (De) of the sample on $2 \mathrm{~mm}$ aliquots. Dose rates were evaluated from the concentrations of the radio-elements $\mathrm{U}$, Th and $\mathrm{K}$ measured by inductively coupled plasma (ICP) instrumentation. Cosmic dose was calculated from current burial depths and final age calculations were performed using DataBase [111].

\section{Appendix B.4. Statistical Evaluation}

The Gradistat for Excel program [54] was used for a statistical assessment of grain sizes, calculating characteristic parameters such as modality, mean grain size, sorting, skewness, and kurtosis [112]. As well, principal component analysis (PCA) and the EMMAgeo v0.9.4 R package for end-member analysis were applied [55]. For the dust samples, PCA and EMMAgeo used laser results with $2 \mu \mathrm{m}$ clay-silt border in order to avoid missing data of medium silt [19].

The EMMAgeo statistical algorith in $\mathrm{R}$, based on the mathematical concept of eigenspace analysis [113], was applied to unmix and describe possible end-members which could represent deposition processes or different source materials [114]. EMMAgeo uses all available sediment samples to identify potential end-members and their contributions to the final archive [55]. All particle size results of both regions were compiled into one matrix for a joint evaluation. In order to avoid empty values of medium silt, we used laser grain size results with the regular clay-silt border of $2 \mu \mathrm{m}$ for statistics with EMMAgeo.

An eigenspace is an attribute space of interrelated processes or sources recorded in a geoarchive. The axes (i.e., eigenvectors) of this space are the underlying processes or sources as linear combinations of measured sediment properties. EMMAgeo identifies end members from the eigenspace of a data set and thus statistically "unmixes" the sources of the sediment. The resulting end members consists of loadings representing the composition in the sample space [115]. Raw grain-size distributions were 
rounded to sum exactly to $100 \%$ before end-member analysis. A transformation of percentage values is necessary [116], as large-scale contrasts may result in weak or hidden correlations. EMMAgeo applies a column-wise weight transformation as suggested by Manson and Imbrie [117] and Klovan and Imbrie [118], and not a Log-ratio transformation as too many zero values may exist within the grain-size distribution space resulting in numerical problems such as artificial extremes and divisions by zero [55]. The true number of final end-members is unknown, but a minimum number of potential end-members can be estimated by testing whether the log-ratios of an error matrix $E$ are normally distributed [119]. EMMAgeo defines the minimum number of potential end-members by an iterative loop taking at least as many eigenvectors into a VARIMAX rotation [120] as needed to explain more than $95 \%$ of total variance in the original data [55].

Principal component analysis (PCA) is a statistical method for dimension reduction of multivariate datasets using orthogonal transformation. It generalizes the dataset by deriving new synthetic variables, so-called principal components that represent the original variables as well as possible [121]. In this study, we used the R-function prcomp [56] to perform the PCA for detecting similarities between samples.

In order to explore whether parameters are suited to distinguish between different types of sediments, we utilized random forest classification. This is a non-parametric classification approach that applies the techniques of decision tree learning and bootstrap aggregation (or bagging), which repeatedly selects a random sample with replacement of the training set and resulting calculated predictions for the samples [122]. Subsequently, the model is evaluated by out-of-bag-validation. Furthermore, the algorithm calculates an importance value for each predictor variable, based on permutation [57]. We used the R-package randomForest to calculate the model. 


\section{Appendix C. Full Results of Analyses}

Table A1. Soil classifications, diagnostic horizons, available ages, color, $\mathrm{pH}$, conductivities, and total organic carbon (TOC) of the Petra region profiles. The sample no. reflects the sample's number used in statistical analyses, ordered according to types of deposits (see Table A8).

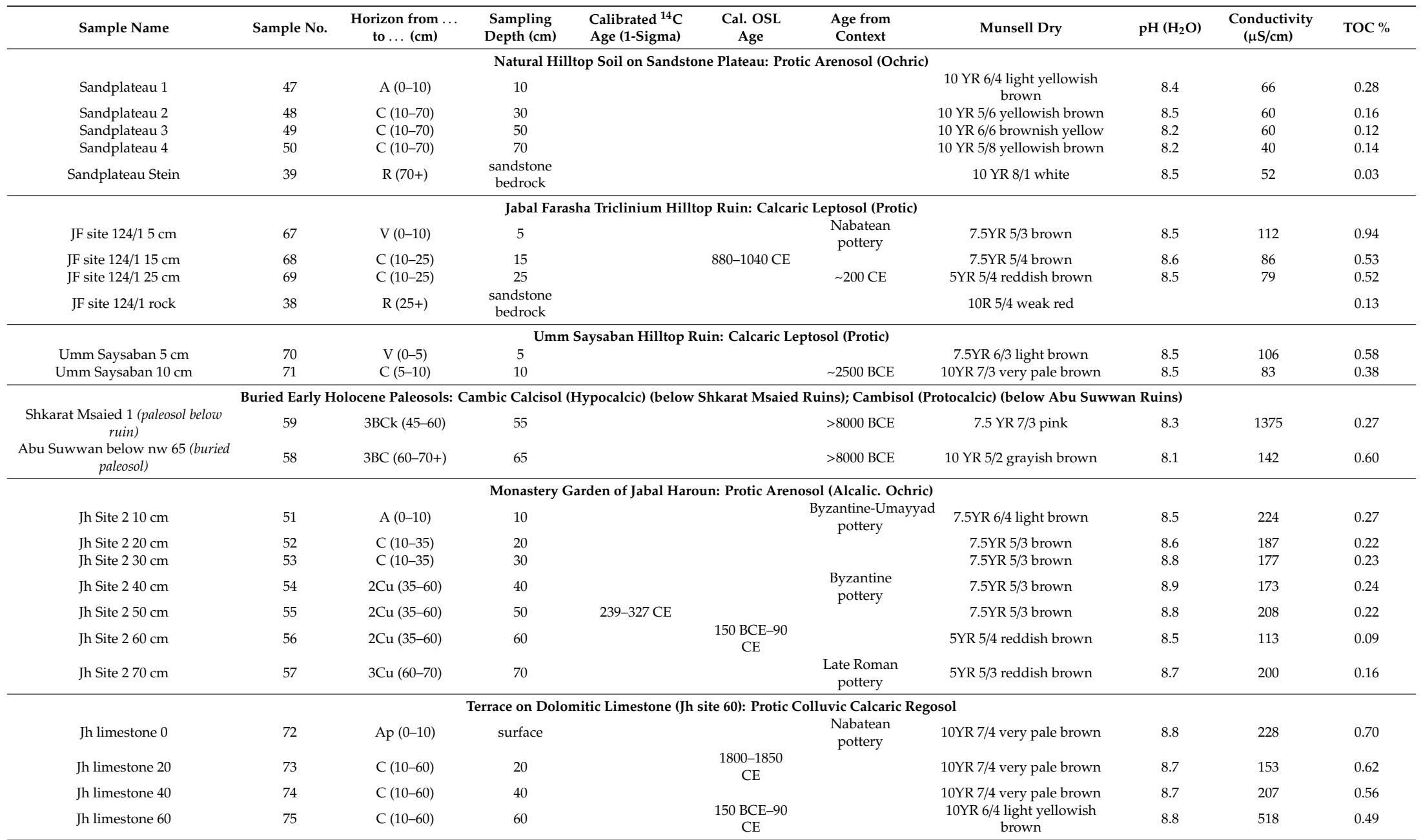


Table A1. Cont.

\begin{tabular}{|c|c|c|c|c|c|c|c|c|c|c|}
\hline Sample Name & Sample No. & $\begin{array}{l}\text { Horizon from } \ldots \\
\quad \text { to } \ldots(\mathrm{cm})\end{array}$ & $\begin{array}{l}\text { Sampling } \\
\text { Depth (cm) }\end{array}$ & $\begin{array}{l}\text { Calibrated }{ }^{14} \mathrm{C} \\
\text { Age (1-Sigma) }\end{array}$ & $\begin{array}{l}\text { Cal. OSL } \\
\text { Age }\end{array}$ & $\begin{array}{c}\text { Age from } \\
\text { Context }\end{array}$ & Munsell Dry & $\mathrm{pH}\left(\mathrm{H}_{2} \mathrm{O}\right)$ & $\begin{array}{c}\text { Conductivity } \\
(\mu \mathrm{S} / \mathrm{cm})\end{array}$ & тос $\%$ \\
\hline \multicolumn{11}{|c|}{ Terrace on Sandstone (Jh site 33): Protic Calcaric Arenosol (Colluvic) over Protic Colluvic Calcaric Regosol } \\
\hline Jh site 3310 & 60 & Ap $(0-20)$ & 10 & & & \multicolumn{2}{|c|}{$\begin{array}{l}\text { Nabatean-LateIslamic } 7.5 Y R \text { 6/4 light brown } \\
\text { pottery }\end{array}$} & 8.8 & 239 & 0.26 \\
\hline Jh site 3330 & 61 & C (20-70) & 30 & & & & 5YR 6/4 light reddish brown & 8.4 & 363 & 0.02 \\
\hline Jh site 3350 & 62 & C (20-70) & 50 & & & & 5YR 6/4 light reddish brown & 8.3 & 374 & 0.01 \\
\hline Jh site 3375 & 63 & $2 \mathrm{C}(70-140)$ & 75 & & & & $5 Y R 4 / 6$ yellowish red & 8.7 & 573 & 0.04 \\
\hline Jh site 33100 & 64 & $2 \mathrm{C}(70-140)$ & 100 & & & & 7.5YR 6/4 light brown & 8.8 & 688 & 0.08 \\
\hline Jh site 33130 & 65 & $2 C(70-140)$ & 130 & & & & 5YR 6/4light reddish brown & 8.7 & 688 & 0.13 \\
\hline Jh site 33150 & 66 & 3C (140-150) & 150 & & & & 5YR 6/4light reddish brown & 8.9 & 599 & 0.10 \\
\hline \multicolumn{11}{|c|}{ Reference Samples from Petra Region: Rocks \& Current Fans } \\
\hline $\mathrm{JH}$ limestone outcrop & 44 & & rock outcrop & & & & 7.5YR $8 / 4$ pink & & & 0.85 \\
\hline Beidha Fan & 46 & & surface & & & & $\begin{array}{l}\text { 10YR 6/4 light yellowish } \\
\text { brown }\end{array}$ & 8.44 & 346 & 0.46 \\
\hline Fan Umm Sayhoun & 45 & & surface & & & & $10 \mathrm{YR} 8 / 4$ very pale brown & 8.98 & 136 & 0.07 \\
\hline JH sandstone (Abu Khushayba) & 41 & & rock outcrop & & & & $2.5 \mathrm{YR} 5 / 3$ reddish brown & 8.7 & 97 & 0.02 \\
\hline Disi Sandstone & 42 & & rock outcrop & & & & 7.5YR $8 / 1$ white & & & 0.14 \\
\hline Um Ishrin Sandstone & 43 & & rock outcrop & & & & 5YR 4/6 yellowish red & & & 0.06 \\
\hline Ba'ja Sandstein & 40 & & surface rock & & & & 10 YR $8 / 2$ white & 8.3 & 81 & 0.02 \\
\hline \multicolumn{11}{|c|}{ Current Dust from Sampler on Top of Jabal Haroun } \\
\hline JH-19-12-16 & 76 & current dust & $\begin{array}{l}\text { Marble } \\
\text { sampler }\end{array}$ & & & 19.12.2016 & n.a. & & & \\
\hline $\mathrm{JH}-07-01-17$ & 77 & current dust & $\begin{array}{l}\text { Marble } \\
\text { sampler }\end{array}$ & & & 07.01.2017 & 10YR 6/3 pale brown & & & \\
\hline JH-15-02-17 & 84 & current dust & $\begin{array}{c}\text { Marble } \\
\text { sampler }\end{array}$ & & & 15.02 .2017 & 10YR $6 / 3$ pale brown & & & \\
\hline $\mathrm{JH}-01-03-17$ & 83 & current dust & $\begin{array}{l}\text { Marble } \\
\text { sampler }\end{array}$ & & & 01.03.2017 & 10YR $5 / 2$ grayish brown & & & \\
\hline $\mathrm{JH}-05-08-17$ & 78 & current dust & $\begin{array}{c}\text { Marble } \\
\text { sampler }\end{array}$ & & & 05.08.2017 & 10YR 5/4 yellowish brown & & & \\
\hline JH-05-08-17 closed box & 79 & current dust & $\begin{array}{c}\text { Marble } \\
\text { sampler }\end{array}$ & & & 05.08.2017 & 10YR 5/4 yellowish brown & & & \\
\hline \multicolumn{11}{|c|}{ Current Dust from Sampler at Foot of of Jabal Haroun } \\
\hline Saleh 25-11-16 & 80 & current dust & $\begin{array}{l}\text { Marble } \\
\text { sampler }\end{array}$ & & & 25.11.2016 & $5 Y R 5 / 3$ reddish brown & & & \\
\hline Saleh 20-06-17 & 81 & current dust & $\begin{array}{l}\text { Marble } \\
\text { sampler }\end{array}$ & & & 20.06 .2017 & 10YR 5/4 yellowish brown & & & \\
\hline Saleh 05-08-17 & 82 & current dust & $\begin{array}{c}\text { Marble } \\
\text { sampler }\end{array}$ & & & 05.08.2017 & 5YR 6/4 light reddish brown & & & \\
\hline
\end{tabular}


Table A2. Soil classifications, diagnostic horizons, available ages, color, $\mathrm{pH}$, conductivities, and total organic carbon (TOC) of the Northern Negev profiles. The sample no. reflects the sample's number used in statistical analyses, ordered according to types of deposits (see Table A8).

\begin{tabular}{|c|c|c|c|c|c|c|c|c|c|c|}
\hline Sample Name & Sample No. & $\begin{array}{l}\text { Horizon from } \ldots \\
\text { to } \ldots(\mathrm{cm})\end{array}$ & $\begin{array}{c}\text { Sampling } \\
\text { Depth (cm) }\end{array}$ & $\begin{array}{l}\text { Calibrated }{ }^{14} \mathrm{C} \\
\text { Age (1-Sigma) }\end{array}$ & $\begin{array}{c}\text { Cal. OSL } \\
\text { Age }\end{array}$ & $\begin{array}{c}\text { Age from } \\
\text { Context }\end{array}$ & Munsell Dry & $\mathrm{pH}\left(\mathrm{H}_{2} \mathrm{O}\right)$ & $\begin{array}{c}\text { Conductivity } \\
(\mu \mathrm{S} / \mathrm{cm})\end{array}$ & TOC \% \\
\hline \multicolumn{11}{|c|}{ Terrace on Chalk in Nahal HaRo'a: Protic Colluvic Calcaric Regosol (Siltic) over Calcaric Fluvisol (Aric, Siltic) over Cambic Calcisol (Siltic) } \\
\hline Haroa Farm $10 \mathrm{C}$ & 7 & $\begin{array}{l}\mathrm{A}(0-5), \mathrm{C}(5-15), \\
2 \mathrm{C}(15-28)\end{array}$ & 10 & & & & $\begin{array}{l}10 \text { YR 6/4 light yellowish } \\
\text { brown }\end{array}$ & 8.1 & 4520 & 3.02 \\
\hline Haroa Farm $28 \mathrm{fAh}$ & 8 & $3 \mathrm{Apb}(28-30)$ & 28 & & & & 10 YR $7 / 4$ very pale brown & 7.8 & 4810 & 1.43 \\
\hline Haroa Farm $40 \mathrm{C}$ & 9 & $3 \mathrm{C}(30-55)$ & 40 & & & & 10 YR $7 / 4$ very pale brown & 8.1 & 4960 & 0.65 \\
\hline Haroa Farm $50 \mathrm{C}$ & 10 & $3 \mathrm{C}(30-55)$ & 50 & & & & 10 YR $7 / 4$ very pale brown & 8.3 & 4650 & 0.55 \\
\hline Haroa Farm $65 \mathrm{C}$ & 11 & $4 \mathrm{C}(55-70)$ & 65 & & & & $10 \mathrm{YR} 7 / 4$ very pale brown & 8.3 & 3420 & 0.59 \\
\hline Haroa Farm 80 B & 12 & $5 \mathrm{Bk}(70-90+)$ & 80 & & & & $\begin{array}{c}10 \text { YR 6/4 light yellowish } \\
\text { brown }\end{array}$ & 8.8 & 2680 & 0.28 \\
\hline \multicolumn{11}{|c|}{ Paleosol in Loessial Apron of tributary of Nahal HaRo'a: Cambic Calcisol (Siltic) } \\
\hline NH-LA-10cm & 5 & $\mathrm{~V}(0-25)$ & 10 & & & & 10YR 8/6 yellow & 8.9 & 111 & 0.43 \\
\hline NH-LA-30cm & 6 & $2 \mathrm{Bwk}(25-55+)$ & 30 & & & & 10YR $6 / 6$ brownish yellow & 9.3 & 135 & 0.38 \\
\hline \multicolumn{11}{|c|}{ Cistern Cleanout Spoil: Protic Calcaric Regosol (Siltic, Transportic) over Calcaric Regosol (Siltic, Transportic) over Camic Calcisol (Siltic) } \\
\hline HH-WW-Cistern1-Cleanout1-40 & 18 & $\mathrm{Cu}(0-80)$ & 40 & & & $\begin{array}{l}\text { Byzantine-Islamic } \\
\text { pottery }\end{array}$ & 10 YR 7/4 very pale brown & 7.7 & 1933 & 0.49 \\
\hline HH-WW-Cistern1-Cleanout1-80 & 19 & $\mathrm{Cu}(0-80)$ & 80 & & & $\begin{array}{l}\text { Byzantine-Islamic } \\
\text { pottery }\end{array}$ & 10 YR $7 / 4$ very pale brown & 7.7 & 1741 & 0.45 \\
\hline HH-WW-Cistern1-Cleanout1-120 & 20 & $2 \mathrm{Cu}(80-160)$ & 120 & & 940-1060 CE & $\begin{array}{l}\text { Byzantine-Islamic } \\
\text { pottery }\end{array}$ & 10 YR $7 / 4$ very pale brown & 8.1 & 1109 & 0.40 \\
\hline HH-WW-Cistern1-Cleanout1-160 & 21 & $2 \mathrm{Cu}(80-160)$ & 160 & & $590-730 \mathrm{CE}$ & $\begin{array}{l}\text { Byzantine-Islamic } \\
\text { pottery }\end{array}$ & 10 YR $7 / 4$ very pale brown & 8.0 & 1445 & 0.38 \\
\hline \multicolumn{11}{|c|}{ Hilltop Ruin Overlooking the Western Wadi: Calcaric Leptosol (Protic, Siltic) } \\
\hline HH-WW R1 10 & 22 & $\mathrm{~V}(0-5), \mathrm{C}(5-20)$ & 10 & & & & 10YR 6/6 brownish yellow & 8.3 & 2300 & 1.10 \\
\hline HH-WW-R1 20 & 23 & $\mathrm{C}(5-20)$ & 20 & & & 2000-2500 BCE & 10YR $5 / 6$ yellowish brown & 8.3 & 3890 & 1.30 \\
\hline \multicolumn{11}{|c|}{$\begin{array}{l}\text { Hilltop Ruin Overlooking the Central Wadi: Protic Calcaric Regosol (Siltic) over Cambic Calcisol (Siltic) } \\
\qquad 1946^{1} \mathrm{CE}(7 \mathrm{~cm})\end{array}$} \\
\hline HH-CW-Ruin 10 & 24 & V0 (0-5), V1 (5-15) & 10 & $\begin{array}{c}\sim 1946^{1} \mathrm{CE}(7 \mathrm{~cm}) \\
1643-1918 \mathrm{CE}(14 \\
\mathrm{cm})\end{array}$ & & & 10YR $7 / 3$ very pale brown & 8.7 & 371 & 0.90 \\
\hline HH-CW-Ruin 25 & 25 & V2 (15-35) & 25 & $\begin{array}{c}1694-1918 \text { CE }(20 \\
\mathrm{cm})\end{array}$ & 870-1020 CE & & 10YR $7 / 3$ very pale brown & 8.4 & 2100 & 0.84 \\
\hline HH-CW-Ruin 50 & 26 & $\mathrm{C}(35-40), 2 \mathrm{C}$ & 50 & & 2570-1990 & & 10YR $7 / 3$ very pale brown & 8.7 & 3450 & 0.76 \\
\hline HH-CW-Ruin 60 & 27 & $2 \mathrm{C}(40-70)$ & 60 & & & & 10YR 7/6 yellow & 9.0 & 3620 & 1.20 \\
\hline HH-CW-Ruin 75 & 28 & $3 \mathrm{Bwk}(70-100+)$ & 75 & & & & 10 YR $7 / 4$ very pale brown & 8.8 & 3320 & 0.30 \\
\hline HH-CW-Ruin 90 & 29 & 3Bwk (70-100+) & 90 & & $\begin{array}{l}\text { 23900-20600 } \\
\text { BCE }\end{array}$ & & 10YR $7 / 4$ very pale brown & 9.0 & 3120 & 1.10 \\
\hline \multicolumn{11}{|c|}{ Western Wadi Terrace 3: Protic Colluvic Calcaric Regosol (Siltic) } \\
\hline HH-WW-T3-20 & 13 & A (0-5), C (0-60) & 20 & & & & 10YR $7 / 4$ Very pale Brown & 8.8 & 265 & 0.60 \\
\hline HH-WW-T3-40 & 14 & $\mathrm{C}(0-60)$ & 40 & & & & 10 YR $7 / 4$ very pale brown & 8.1 & 164 & 0.43 \\
\hline HH-WW-T3-70 & 15 & $2 \mathrm{C}(60-120)$ & 70 & & $320-480 \mathrm{CE}$ & & 10YR 7/4 Very pale Brown & 8.5 & 107 & 0.32 \\
\hline HH-WW-T3-90 & 16 & $2 \mathrm{C}(60-120)$ & 90 & 334-94 BCE & & & $10 Y R$ R $7 / 3$ very pale brown & 8.1 & 223 & 0.33 \\
\hline HH-WW-T3-120 & 17 & $2 \mathrm{C}(60-120)$ & 120 & & $460-240 \mathrm{BCE}$ & & $10 Y R$ R $7 / 3$ very pale brown & 8.0 & 127 & 0.24 \\
\hline
\end{tabular}


Table A2. Cont.

\begin{tabular}{|c|c|c|c|c|c|c|c|c|c|c|}
\hline Sample Name & Sample No. & $\begin{array}{l}\text { Horizon from ... } \\
\text { to } \ldots \text { (cm) }\end{array}$ & $\begin{array}{c}\text { Sampling } \\
\text { Depth (cm) }\end{array}$ & $\begin{array}{l}\text { Calibrated }{ }^{14} \mathrm{C} \\
\text { Age (1-Sigma) }\end{array}$ & $\begin{array}{c}\text { Cal. OSL } \\
\text { Age }\end{array}$ & $\begin{array}{c}\text { Age from } \\
\text { Context }\end{array}$ & Munsell Dry & $\mathrm{pH}\left(\mathrm{H}_{2} \mathrm{O}\right)$ & $\begin{array}{c}\text { Conductivity } \\
(\mu \mathrm{S} / \mathrm{cm})\end{array}$ & TOC \% \\
\hline \multicolumn{11}{|c|}{ Negev reference samples } \\
\hline Haroa Farm chalk & 4 & chalk & outcrop & & & & 5YR $8 / 2$ pinkish white & & & \\
\hline HH-WW-C2-soft limestone & 2 & soft limestone & outcrop & & & & 5YR 8/1 white & & & \\
\hline HH-WW-C2-hard limestone & 90 & hard limestone & outcrop & & & & 5YR $8 / 1$ white & & & \\
\hline HH-CW-chalk & 3 & chalk & outcrop & & & & 7.5YR $8 / 3$ pink & & & \\
\hline HH-CW-Tur-Paleo & 1 & Turonian paleosol & outcrop & & & & $2.5 Y 7 / 4$ pale yellow & 7.6 & 1817 & 0.52 \\
\hline \multicolumn{11}{|c|}{ Current Dust from Midreshet Ben Gurion, Sede Boker } \\
\hline 25-03-03-HH dry spot & - & current dust & & & & & 10YR $7 / 6$ yellow & & & \\
\hline 25-03-03-HH-incl-washout & 30 & current dust & & & & & 10YR 7/6 yellow & & & \\
\hline $11-12-10-\mathrm{HH}$ & 31 & current dust & & & & & $10 Y R \quad 7 / 4$ very pale brown & & & \\
\hline $12-12-10-\mathrm{HH}$ & 32 & current dust & & & & & $10 Y R 7 / 4$ very pale brown & & & \\
\hline 29-02-12-HH & 33 & current dust & & & & & $10 Y R \quad 7 / 4$ very pale brown & & & \\
\hline $18-04-12-\mathrm{HH}$ & 34 & current dust & & & & & $10 Y R$ R $7 / 4$ very pale brown & & & \\
\hline 20-12-12-HH & 35 & current dust & & & & & & & & \\
\hline 22-03-13-HH & 36 & current dust & & & & & $10 Y R \quad 7 / 4$ very pale brown & & & \\
\hline $11-02-15-\mathrm{HH}$ & 37 & current dust & & & & & $10 \mathrm{YR} 7 / 4$ very pale brown & & & \\
\hline $01-12-16-\mathrm{HH}$ & - & current dust & & & & & & & & \\
\hline $05-01-18-\mathrm{HH}$ & - & current dust & & & & & 10YR $7 / 6$ yellow & & & \\
\hline 28-03-18-HH & - & current dust & & & & & $10 Y R 7 / 6$ yellow & & & \\
\hline
\end{tabular}

Table A3. OSL-dating results.

\begin{tabular}{|c|c|c|c|c|c|c|c|c|c|c|c|c|c|c|}
\hline \multirow{2}{*}{ Lab Code } & \multirow{2}{*}{ Sample } & \multirow{2}{*}{$\begin{array}{l}\text { Depth Below } \\
\text { Surface }[\mathrm{cm}]\end{array}$} & \multirow{2}{*}{$\begin{array}{c}\text { Water } \\
\%\end{array}$} & \multirow{2}{*}{$\begin{array}{c}\text { Th } \\
\text { ppm }\end{array}$} & \multirow{2}{*}{$\begin{array}{c}\mathrm{U} \\
\mathrm{ppm}\end{array}$} & \multirow{2}{*}{$\begin{array}{l}\mathbf{K} \\
\%\end{array}$} & \multirow{2}{*}{$\begin{array}{c}\text { Dose Rate } \\
\text { Gy/ka }\end{array}$} & \multirow{2}{*}{$\begin{array}{l}\text { OD } \\
(\%)\end{array}$} & \multirow[t]{2}{*}{$\mathbf{N}$} & \multicolumn{2}{|c|}{ De } & \multicolumn{2}{|c|}{ Age } & \multirow{2}{*}{$\begin{array}{c}\text { Year AD/BC } \\
\text { yrs }\end{array}$} \\
\hline & & & & & & & & & & Gy & \pm & yrs & \pm & \\
\hline HLK-2 & HH Cistern1 Cleanout1 110 & 110 & $8 \pm 3$ & 6.3 & 1.8 & 0.90 & $1.79 \pm 0.07$ & 30 & $17 / 19$ & 1.84 & 0.08 & 1020 & 60 & $940-1060 \mathrm{CE}$ \\
\hline HLK-1 & HH Cistern1 Cleanout1 150 & 150 & $8 \pm 3$ & 5.4 & 1.6 & 0.78 & $1.57 \pm 0.06$ & 13 & $18 / 18$ & 2.13 & 0.07 & 1360 & 70 & $590-730 \mathrm{CE}$ \\
\hline HLK-12 & HH-CW-Ruin 25 & 25 & $5 \pm 3$ & 4.7 & 1.5 & 1.08 & $1.82 \pm 0.07$ & 35 & $30 / 31$ & 1.96 & 0.12 & 1070 & 80 & $870-1020 \mathrm{CE}$ \\
\hline HLK-11 & HH-CW-Ruin 50 & 55 & $8 \pm 3$ & 3.4 & 1.3 & 0.73 & $1.36 \pm 0.05$ & 34 & $32 / 33$ & 5.9 & 0.4 & 4300 & 290 & 2570-1990 BCE \\
\hline HLK-10 & HH-CW-Ruin 90 & 90 & $8 \pm 3$ & 6.3 & 2.1 & 1.31 & $2.21 \pm 0.09$ & 32 & $17 / 19$ & 54 & 3 & 24300 & 1700 & 23900-20600 BCE \\
\hline HKL-4 & HH-WW-T3-70 & 70 & $8 \pm 3$ & 5.3 & 1.8 & 0.79 & $1.64 \pm 0.06$ & 11 & $19 / 19$ & 2.67 & 0.08 & 1620 & 80 & $320-480$ CE \\
\hline HKL-3 & HH-WW-T3-100 & 100 & $8 \pm 3$ & 4.3 & 1.4 & 0.57 & $1.28 \pm 0.05$ & 11 & $19 / 19$ & 3.02 & 0.09 & 2370 & 110 & $460-240 \mathrm{BCE}$ \\
\hline PET-20 & JF site 124/1 15 & 15 & $5 \pm 2$ & 10.2 & 2.42 & 0.56 & $2.02 \pm 0.07$ & 27 & $19 / 19$ & 2.15 & 0.14 & 1060 & 80 & $880-1040 \mathrm{CE}$ \\
\hline PET-19 & Jh limestone 20 & 20 & $5 \pm 2$ & 4.4 & 1.6 & 0.58 & $1.45 \pm 0.04$ & 67 & $28 / 30$ & 0.28 & 0.03 & 200 & 20 & $1800-1850 \mathrm{CE}$ \\
\hline PET-18 & Jh limestone 50 & 50 & $8 \pm 3$ & 3.3 & 1.3 & 0.36 & $1.04 \pm 0.04$ & 74 & MAM * & 2.28 & 0.21 & 2180 & 210 & 340 BCE-50 CE \\
\hline PET-9 & $\mathrm{JH}$ site $260 \mathrm{~cm}$ & 60 & $5 \pm 2$ & 4.9 & 1.8 & 0.16 & $1.14 \pm 0.04$ & 26 & $\begin{array}{r}17 / 19 \\
17\end{array}$ & 2.28 & 0.10 & 2050 & 120 & 150 BCE-90 CE \\
\hline
\end{tabular}

Notes: OSL ages on 90-125 $\mu \mathrm{m}$ quartz samples. DR = dose rate, including cosmic dose. De = Equivalent dose. Errors on radioelements: Th- $10 \%$; U-8\%; K-5\%. OSL De values and errors calculated using the central age model. OD—over-dispersion, a measure of the scatter in a sample beyond that expected from instrumental errors. $\mathrm{N}-$ number of aliquots used for age calculations out of those measured. * age calculated using the Minimum Age Model. 
Table A4. ${ }^{14}$ C-dating results.

\begin{tabular}{|c|c|c|c|c|c|c|c|c|}
\hline \multirow{2}{*}{ Labcode } & \multirow{2}{*}{ Sample } & \multirow{2}{*}{ Material } & \multicolumn{2}{|c|}{$\mathrm{C} 14$} & \multirow{2}{*}{$\frac{\text { cal BCE/CE }}{1 \sigma}$} & \multirow{2}{*}{$\frac{\text { cal BCE/CE }}{2 \sigma}$} & \multirow{2}{*}{$\mathrm{C}(\%)$} & \multirow{2}{*}{ Software/Dataset } \\
\hline & & & yrs BP & \pm & & & & \\
\hline UBA-38659 & HH-CW-R 13-15 & charcoal & 229 & 35 & $1643-1949 * \mathrm{CE}$ & $1527-1949 * \mathrm{CE}$ & 76.8 & CALIB7.04/IntCal13 \\
\hline UBA-38660 & HH-WC-R-20 & charcoal & 106 & 27 & 1694-1918 CE & 1682-1935 CE & 75.7 & CALIB7.04/IntCal13 \\
\hline UBA-38661 & HH-WC-R-5-8 & charcoal & 4 & 24 & \multicolumn{2}{|c|}{ age- 2 sigma outside calibration age range } & 82.8 & \\
\hline UBA-34166 & $\mathrm{HH}-\mathrm{WW}-3-90 \mathrm{~cm}$ & charcoal & 2130 & 36 & 334-94 BCE & $352-49$ ВCE & 61.6 & CALIB7.04/IntCal13 \\
\hline UBA-38662 & Jh site $250 \mathrm{~cm}$ & charcoal & 1763 & 27 & 239-327 CE & 173-379 CE & 73.5 & CALIB7.04/IntCal13 \\
\hline
\end{tabular}

Table A5. Average sedimentation rates re-calculated for all archaeological soils (see Lucke et al. [19] for hilltop ruins). The following experimentally determined bulk densities were used: $0.901 \mathrm{~g} / \mathrm{cm}^{3}$ (hilltop ruins), 1.036/0.895 g/ $\mathrm{cm}^{3}$ (compact/friable terrace sediments), 1.296/0.954 g/ $\mathrm{cm}^{3}$ (compact/friable cistern cleanout spoil).

\begin{tabular}{cccc}
\hline Site & mm/year & $\mathrm{g} / \mathbf{m}^{\mathbf{2}}$ year $\mathbf{~}^{-1}$ & Comment \\
\hline Monastery garden (Jh site 2) & 0.29 & Petra region & Steady and continuous sedimentation, apparently ongoing \\
Limestone terrace (Jh site 60) & 0.27 & 264 & $\begin{array}{c}\text { Strongly scattering OSL-ages, samples may consist of mixtures of } \\
\text { older and younger material }\end{array}$ \\
Jabal Farasha triclinium & 0.14 & 246 & Apparently constant deposition; consistent ages \\
Umm Saysaban & 0.02 & 125 & Minimum rate: ruin filled completely in the past \\
Jabal Haroun monastery & 0.10 & 20 & Minimum rate: lower boundary unclear \\
Cistern Cleanout & & 90 & Lower, compact part of cleanout pile that was dated by OSL \\
Terrace 3 & 1.47 & 1906 & Possibly inhomogeneous sedimentation rates \\
Ruin near Western Wadi & 0.51 & $453-525$ & Minimum rate: ruin filled completely in the past \\
Ruin near Central Wadi & 0.05 & 42 & Lower boundary unclear; occupation \& collapse layers? \\
\hline
\end{tabular}


Table A6. $\mathrm{CaCO}_{3}$-contents, grain sizes, and statistical parameters from the Petra region. Dust samples were measured by laser grain size analysis with assumed clay-silt border of $5 \mu \mathrm{m}$ for optimal comparability with Sedigraph results [11,19]. Note that the laser medium silt fraction is mathematically eliminated due to the adapted clay border. The sample no. reflects the sample's number used in statistical analyses, ordered according to types of deposits (see Table A8).

\begin{tabular}{|c|c|c|c|c|c|c|c|c|c|c|c|c|c|c|c|c|c|c|c|}
\hline Sample Name & $\begin{array}{c}\text { Sample } \\
\text { No. }\end{array}$ & $\underset{\%}{\mathrm{CaCO}_{3}}$ & $\begin{array}{l}\text { Skeleton > } \\
2 \mathrm{~mm}(\%)\end{array}$ & $\begin{array}{r}\text { Coarse } \\
\text { Sand \% }\end{array}$ & $\begin{array}{c}\text { Medium } \\
\text { Sand \% }\end{array}$ & $\begin{array}{c}\text { Fine } \\
\text { Sand \% }\end{array}$ & $\begin{array}{l}\text { Coarse } \\
\text { Silt \% }\end{array}$ & $\begin{array}{c}\text { Medium } \\
\text { Silt } \% \\
\end{array}$ & $\begin{array}{c}\text { Fine Silt } \\
\% \\
\end{array}$ & $\begin{array}{l}\text { Coarse } \\
\text { Clay \% }\end{array}$ & $\begin{array}{c}\text { Medium } \\
\text { Clay \% }\end{array}$ & $\begin{array}{l}\text { Fine } \\
\text { Clay } \%\end{array}$ & $\begin{array}{c}\text { Sand } \\
\%\end{array}$ & $\begin{array}{c}\text { Silt } \\
\%\end{array}$ & $\begin{array}{c}\text { Clay } \\
\%\end{array}$ & $\begin{array}{c}\text { MODE } 1 \\
{[\mu \mathrm{m}]}\end{array}$ & $\begin{array}{c}\text { MODE } 2 \\
{[\mu \mathrm{m}]}\end{array}$ & $\begin{array}{c}\text { MODE } 3 \\
{[\mu \mathrm{m}]}\end{array}$ & $\begin{array}{c}\text { MEAN } \\
{[\mu \mathrm{m}]}\end{array}$ \\
\hline \multicolumn{20}{|c|}{ Natural Hilltop Soil on Sandstone Plateau: Protic Arenosol (Ochric) } \\
\hline Sandplateau 1 & 47 & 2 & 0 & 3 & 59 & 18 & 6 & 4 & 3 & 3 & 2 & 2 & 80 & 13 & 8 & 415 & 0 & 0 & 143 \\
\hline Sandplateau 2 & 48 & 1 & 5 & 2 & 61 & 19 & 4 & 3 & 3 & 3 & 3 & 3 & 82 & 9 & 9 & 415 & 0 & 0 & 169 \\
\hline Sandplateau 3 & 49 & 0 & 9 & 2 & 61 & 19 & 4 & 3 & 3 & 3 & 3 & 3 & 82 & 9 & 9 & 415 & 0 & 0 & 161 \\
\hline Sandplateau 4 & 50 & 0 & 0 & 1 & 62 & 18 & 3 & 3 & 3 & 3 & 3 & 4 & 82 & 8 & 10 & 415 & 0 & 0 & 160 \\
\hline Sandplateau Stein (bedrock) & 39 & 0 & 0 & 1 & 75 & 12 & 2 & 2 & 4 & 3 & 1 & 0 & 89 & 7 & 4 & 415 & 0 & 0 & 248 \\
\hline \multicolumn{20}{|c|}{ Jabal Farasha Triclinium Hilltop Ruin: Calcaric Leptosol (Protic) } \\
\hline $\mathrm{JF}$ site $124 / 15 \mathrm{~cm}$ & 67 & 13 & 3 & 3 & 17 & 30 & 18 & 11 & 8 & 6 & 4 & 3 & 51 & 36 & 13 & 132 & 0 & 0 & 38 \\
\hline $\mathrm{JF}$ site $124 / 15 \mathrm{~cm} \mathrm{S \& C}$ & 85 & 22 & 0 & 0 & 0 & 0 & 37 & 22 & 15 & 12 & 8 & 6 & 0 & 74 & 26 & 42 & 0 & 0 & 7 \\
\hline $\mathrm{JF}$ site $124 / 115 \mathrm{~cm}$ & 68 & 10 & 5 & 3 & 16 & 34 & 19 & 9 & 7 & 6 & 3 & 3 & 53 & 34 & 12 & 132 & 0 & 0 & 41 \\
\hline JF site $124 / 125 \mathrm{~cm}$ & 69 & 8 & 21 & 4 & 14 & 39 & 15 & 8 & 6 & 6 & 5 & 2 & 57 & 29 & 14 & 132 & 1 & 0 & 38 \\
\hline $\mathrm{JF}$ site $124 / 1$ bedrock & 38 & 2 & n.a. & 48 & 10 & 2 & 3 & 8 & 8 & 10 & 9 & 3 & 60 & 18 & 22 & 1315 & 1 & 13 & 89 \\
\hline \multicolumn{20}{|c|}{ Umm Saysaban Hilltop Ruin: Calcaric Leptosol (Protic) } \\
\hline Umm Saysaban $5 \mathrm{~cm}$ & 70 & 12 & 13 & 3 & 16 & 32 & 15 & 11 & 9 & 8 & 4 & 2 & 51 & 35 & 14 & 132 & 0 & 0 & 35 \\
\hline Umm Saysaban $10 \mathrm{~cm}$ & 71 & 12 & 12 & 5 & 18 & 32 & 13 & 9 & 8 & 8 & 5 & 3 & 54 & 30 & 16 & 132 & 0 & 0 & 36 \\
\hline Umm Saysaban $10 \mathrm{~cm} \mathrm{S \& C}$ & 86 & 25 & 0 & 0 & 0 & 0 & 29 & 19 & 18 & 18 & 11 & 6 & 0 & 66 & 34 & 42 & 0 & 0 & 5 \\
\hline \multicolumn{20}{|c|}{ Buried Early Holocene Paleosols: Cambic Calcisol (Hypocalcic) (below Shkarat Msaied Ruins); Cambisol (Protocalcic) (below Abu Suwwan Ruins) } \\
\hline Shakarat Msaid 1 & 59 & 15 & 0 & 0 & 30 & 21 & 11 & 7 & 8 & 8 & 6 & 8 & 52 & 26 & 22 & 415 & 4 & 0 & 27 \\
\hline Shakarat Msaid $1 \mathrm{S \& C}$ & 88 & 30 & 0 & 0 & 0 & 0 & 23 & 15 & 17 & 17 & 13 & 16 & 0 & 54 & 46 & 42 & 4 & 0 & 2 \\
\hline Abu Suwwan below nw 65 & 58 & 18 & 2 & 1 & 39 & 22 & 7 & 8 & 8 & 7 & 5 & 3 & 62 & 23 & 15 & 415 & 4 & 0 & 48 \\
\hline \multicolumn{20}{|c|}{ Monastery Garden of Jabal Haroun: Protic Arenosol (Alcalic. Ochric) } \\
\hline Jh Site $210 \mathrm{~cm}$ & 51 & 4 & 2 & 2 & 63 & 19 & 3 & 2 & 2 & 2 & 3 & 4 & 83 & 7 & 9 & 415 & 0 & 0 & 201 \\
\hline Jh Site $220 \mathrm{~cm}$ & 52 & 3 & 5 & 2 & 63 & 21 & 2 & 1 & 2 & 2 & 3 & 4 & 86 & 5 & 9 & 415 & 0 & 0 & 208 \\
\hline Jh Site $230 \mathrm{~cm}$ & 53 & 3 & 12 & 2 & 54 & 24 & 3 & 2 & 2 & 3 & 5 & 5 & 80 & 7 & 13 & 415 & 0 & 0 & 106 \\
\hline Jh Site $240 \mathrm{~cm}$ & 54 & 3 & 13 & 0 & 50 & 25 & 3 & 2 & 3 & 4 & 6 & 7 & 75 & 9 & 17 & 415 & 0 & 0 & 51 \\
\hline Jh Site $250 \mathrm{~cm}$ & 55 & 3 & 4 & 1 & 54 & 25 & 2 & 2 & 2 & $\begin{array}{l}4 \\
4\end{array}$ & 5 & 5 & 79 & 7 & 13 & 415 & 0 & 0 & 89 \\
\hline Jh Site $260 \mathrm{~cm}$ & 56 & 3 & $\begin{array}{l}7 \\
9\end{array}$ & 2 & 60 & 22 & 1 & 1 & 2 & $\begin{array}{l}4 \\
3\end{array}$ & 4 & 5 & 83 & 5 & 12 & 415 & 0 & 0 & 167 \\
\hline Jh Site $270 \mathrm{~cm}$ & 57 & 4 & 22 & 2 & 52 & 20 & 5 & 2 & 2 & 4 & 5 & 8 & 74 & 9 & 17 & 415 & 0 & 0 & 53 \\
\hline \multicolumn{20}{|c|}{ Terrace on Dolomitic Limestone (Jh site 60): Protic Colluvic Calcaric Regosol } \\
\hline Jh limestone 0 & 72 & 55 & 20 & 6 & 8 & 22 & 19 & 14 & 12 & 11 & 5 & 3 & 36 & 45 & 19 & 132 & 0 & 0 & 19 \\
\hline Jh limestone 20 & 73 & 55 & 17 & 3 & 4 & 25 & 19 & 15 & 13 & 11 & 6 & 4 & 32 & 47 & 21 & 132 & 0 & 0 & 15 \\
\hline Jh limestone 40 & 74 & 58 & 30 & 6 & 7 & 21 & 14 & 14 & 13 & 12 & 8 & 5 & 34 & 41 & 25 & 132 & 13 & 0 & 13 \\
\hline Jh limestone 60 & 75 & $\begin{array}{l}30 \\
55\end{array}$ & 18 & 4 & 6 & $\begin{array}{l}21 \\
19\end{array}$ & 17 & $\begin{array}{l}14 \\
13\end{array}$ & 14 & 12 & $\frac{0}{7}$ & 8 & 30 & $\begin{array}{l}41 \\
43\end{array}$ & 28 & 132 & 4 & 0 & $\begin{array}{l}10 \\
11\end{array}$ \\
\hline Jh limestone $60 \mathrm{S \& C}$ & 89 & 51 & 0 & 0 & 0 & 0 & 24 & 19 & 20 & 17 & 10 & 11 & 0 & 61 & 40 & 42 & 4 & 0 & 3 \\
\hline \multicolumn{20}{|c|}{ Terrace on Sandstone (Jh site 33): Protic Calcaric Arenosol (Colluvic) over Protic Colluvic Calcaric Regosol } \\
\hline Jh site 3310 & 60 & 39 & 26 & 11 & 20 & 35 & 13 & & 7 & 4 & 1 & 1 & 66 & 27 & 7 & 132 & 0 & 0 & 79 \\
\hline Jh site 3330 & 61 & 11 & 6 & 4 & 25 & 45 & 7 & 4 & 4 & 4 & 3 & 5 & 73 & 15 & 12 & 132 & 0 & 0 & 65 \\
\hline Jh site 3350 & 62 & 7 & 3 & 3 & 21 & 50 & 7 & 4 & 4 & 4 & 3 & 4 & 74 & 15 & 11 & 132 & 0 & 0 & 66 \\
\hline Jh site 3375 & 63 & 15 & 1 & 0 & 2 & 30 & 17 & $\begin{array}{c}7 \\
12\end{array}$ & 11 & 9 & 8 & 11 & 32 & 41 & 27 & 132 & 0 & 0 & 10 \\
\hline Jh site 33100 & 64 & 23 & 0 & 0 & 3 & 13 & 10 & 16 & 19 & 13 & 6 & 20 & 16 & 45 & 39 & 4 & 132 & 0 & 3 \\
\hline Jh site $33100 \mathrm{S \& C}$ & 87 & 26 & 0 & 0 & 0 & 0 & 12 & 19 & 23 & 15 & 7 & 24 & 0 & 54 & 46 & 4 & 0 & 0 & 1 \\
\hline Jh site 33130 & 65 & 28 & 3 & 2 & 10 & 20 & 7 & 12 & 16 & 14 & 8 & 11 & 31 & 36 & 33 & 132 & 4 & 0 & 8 \\
\hline Jh site 33150 & 66 & 24 & 8 & 5 & 19 & 35 & 6 & 7 & 10 & 8 & 5 & 5 & 59 & 23 & 18 & 132 & 4 & 0 & 35 \\
\hline
\end{tabular}


Table A6. Cont.

\begin{tabular}{|c|c|c|c|c|c|c|c|c|c|c|c|c|c|c|c|c|c|c|c|}
\hline Sample Name & $\begin{array}{c}\text { Sample } \\
\text { No. }\end{array}$ & $\begin{array}{c}\mathrm{CaCO}_{3} \\
\% \\
\end{array}$ & $\begin{array}{l}\text { Skeleton > } \\
2 \mathrm{~mm}(\%)\end{array}$ & $\begin{array}{c}\text { Coarse } \\
\text { Sand } \% \\
\end{array}$ & $\begin{array}{l}\text { Medium } \\
\text { Sand \% } \\
\end{array}$ & $\begin{array}{c}\text { Fine } \\
\text { Sand } \%\end{array}$ & $\begin{array}{l}\text { Coarse } \\
\text { Silt \% } \\
\end{array}$ & $\begin{array}{c}\text { Medium } \\
\text { Silt \% }\end{array}$ & $\begin{array}{c}\text { Fine Silt } \\
\%\end{array}$ & $\begin{array}{l}\text { Coarse } \\
\text { Clay \% } \\
\end{array}$ & $\begin{array}{l}\text { Medium } \\
\text { Clay } \%\end{array}$ & $\begin{array}{c}\text { Fine } \\
\text { Clay } \% \\
\end{array}$ & $\begin{array}{c}\text { Sand } \\
\%\end{array}$ & $\begin{array}{c}\text { Silt } \\
\%\end{array}$ & $\begin{array}{c}\text { Clay } \\
\%\end{array}$ & $\begin{array}{c}\text { MODE } 1 \\
{[\mu \mathrm{m}]}\end{array}$ & $\begin{array}{c}\text { MODE } 2 \\
{[\mu \mathrm{m}]}\end{array}$ & $\begin{array}{c}\text { MODE } 3 \\
{[\mu \mathrm{m}]}\end{array}$ & $\begin{array}{c}\text { MEAN } \\
{[\mu \mathrm{m}]}\end{array}$ \\
\hline \multicolumn{20}{|c|}{ Reference Samples from Petra Region: Rocks \& Current Fans } \\
\hline Jh limestone outcrop & 44 & 98 & n.a. & 29 & 14 & 5 & 12 & 27 & 7 & 2 & 1 & 2 & 48 & 46 & 6 & 1315 & 13 & 0 & 74 \\
\hline Beidha Fan & 46 & 16 & 9 & 3 & 43 & 22 & 9 & 5 & 6 & 7 & 3 & 1 & 69 & 21 & 11 & 415 & 1 & 0 & 72 \\
\hline Fan Umm Sayhoun & 45 & 5 & 7 & 16 & 66 & 9 & 0 & 1 & 1 & 2 & 1 & 3 & 91 & 3 & 6 & 415 & 0 & 0 & 338 \\
\hline JH sandstone (Abu & 41 & 2 & n.a. & 3 & 63 & 13 & 2 & 3 & 5 & 5 & 2 & 2 & 79 & 11 & 10 & 415 & 0 & 0 & 101 \\
\hline $\begin{array}{l}\text { Khushayaba) } \\
\text { Disi Sandstone }\end{array}$ & 42 & 4 & n.a. & 11 & 67 & 12 & 1 & 2 & 2 & 2 & 1 & 1 & 91 & 6 & 4 & 415 & 0 & 0 & 281 \\
\hline Um Ishrin & 43 & 2 & n.a. & 18 & 55 & 9 & 3 & 4 & 4 & 3 & 1 & 2 & 82 & 12 & 7 & 415 & 0 & 0 & 186 \\
\hline Ba'ja Sandstein & 40 & 1 & n.a. & 14 & 31 & 27 & 3 & 3 & 6 & 17 & 0 & 0 & 72 & 11 & 17 & 415 & 1 & 0 & 56 \\
\hline \multicolumn{20}{|c|}{ Current Dust from Sampler on Summit of Jabal Haroun } \\
\hline JH-19-12-16 & 76 & 3 & 0 & 0 & 36 & 54 & 4 & n.a. & 4 & 3 & 0 & 0 & 89 & 8 & 3 & 132 & 0 & 0 & 157 \\
\hline JH-07-01-17 & 77 & 6 & 0 & 10 & 62 & 11 & 7 & n.a. & 6 & 4 & 0 & 0 & 83 & 13 & 4 & 415 & 0 & 0 & 204 \\
\hline $\mathrm{JH}-15-02-17$ (snow) & 84 & 15 & 0 & 1 & 24 & 19 & 22 & $\begin{array}{l}\text { n.a. } \\
\text { n.a. }\end{array}$ & 21 & 13 & 0 & 0 & 44 & 43 & 13 & 415 & 42 & 10 & 34 \\
\hline JH-01-03-17 (rain) & $\begin{array}{l}07 \\
83\end{array}$ & 11 & 0 & 0 & 11 & 35 & 30 & $\begin{array}{l}\text { n.a. } \\
\text { n.a. }\end{array}$ & 16 & 7 & 0 & 0 & 46 & 46 & 8 & 132 & 0 & 0 & 40 \\
\hline JH-05-08-17 & 78 & 5 & 0 & 4 & 63 & 14 & 7 & $\begin{array}{l}\text { l.a. } \\
\text { n.a. }\end{array}$ & 7 & 5 & 0 & 0 & 80 & $\begin{array}{l}40 \\
14\end{array}$ & 6 & 415 & 0 & 0 & 170 \\
\hline JH-05-08-17 (dust devil?) & 79 & 4 & 0 & 3 & 65 & 13 & 6 & n.a. & 6 & 5 & 0 & 0 & 82 & 13 & 5 & 415 & 0 & 0 & 182 \\
\hline \multicolumn{20}{|c|}{ Current Dust from Sampler at Foot of of Jabal Haroun } \\
\hline Saleh 25-11-16 & 80 & 2 & 0 & 0 & 52 & 34 & 5 & n.a. & 5 & 4 & 0 & 0 & 86 & 10 & 4 & 415 & 0 & 0 & 184 \\
\hline Saleh 20-06-17 & 81 & 12 & 0 & 0 & 19 & 41 & 23 & $\begin{array}{l}\text { n.a. } \\
\text { n.a. }\end{array}$ & 10 & 8 & 0 & 0 & 59 & 33 & 8 & 132 & 1 & 0 & 63 \\
\hline Saleh 05-08-17 & 82 & 11 & 0 & 1 & 23 & 42 & 19 & n.a. & 8 & 6 & 0 & 0 & 65 & 28 & 7 & 132 & 1 & 0 & 85 \\
\hline
\end{tabular}


Table A7. $\mathrm{CaCO}_{3}$-contents, grain sizes, and some statistical parameters from the Northern Negev. Dust samples were measured by laser grain size analysis with assumed clay-silt border of $5 \mu \mathrm{m}$ for optimal comparability with Sedigraph results [11,19]. Note that laser medium silt fraction is mathematically eliminated due to the adapted clay border. The sample no. reflects the sample's number used in statistical analyses, ordered according to types of deposits (see Table A8).

\begin{tabular}{|c|c|c|c|c|c|c|c|c|c|c|c|c|c|c|c|c|c|c|c|}
\hline Sample Name & $\begin{array}{c}\text { Sample } \\
\text { No. }\end{array}$ & $\begin{array}{c}\mathrm{CaCO}_{3} \\
\%\end{array}$ & $\begin{array}{l}\text { Skeleton > } \\
2 \mathrm{~mm}(\%)\end{array}$ & $\begin{array}{r}\text { Coarse } \\
\text { Sand \% } \\
\end{array}$ & $\begin{array}{l}\text { Medium } \\
\text { Sand \% }\end{array}$ & $\begin{array}{c}\text { Fine } \\
\text { Sand \% }\end{array}$ & $\begin{array}{l}\text { Coarse } \\
\text { Silt \% } \\
\end{array}$ & $\begin{array}{c}\text { Medium } \\
\text { Silt } \% \\
\end{array}$ & $\begin{array}{c}\text { Fine Silt } \\
\% \\
\end{array}$ & $\begin{array}{l}\text { Coarse } \\
\text { Clay \% } \\
\end{array}$ & $\begin{array}{l}\text { Medium } \\
\text { Clay } \%\end{array}$ & $\begin{array}{c}\text { Fine } \\
\text { Clay } \% \\
\end{array}$ & $\begin{array}{c}\text { Sand } \\
\%\end{array}$ & $\begin{array}{c}\text { Silt } \\
\%\end{array}$ & $\begin{array}{c}\text { Clay } \\
\%\end{array}$ & $\begin{array}{c}\text { MODE } 1 \\
{[\mu \mathrm{m}]}\end{array}$ & $\begin{array}{c}\text { MODE } 2 \\
{[\mu \mathrm{m}]}\end{array}$ & $\begin{array}{c}\text { MODE } 3 \\
{[\mu \mathrm{m}]}\end{array}$ & $\begin{array}{c}\text { MEAN } \\
{[\mu \mathrm{m}]}\end{array}$ \\
\hline \multicolumn{20}{|c|}{ Terrace on Chalk in Nahal HaRo'a: Protic Colluvic Calcaric Regosol (Siltic) over Calcaric Fluvisol (Aric, Siltic) over Cambic Calcisol (Siltic) } \\
\hline Haroa Farm $10 \mathrm{C}$ & 7 & 24 & 3 & 3 & 2 & 22 & 34 & 15 & 12 & 7 & 3 & 3 & 26 & & 12 & 42 & 0 & 0 & 21 \\
\hline Haroa Farm $28 \mathrm{fAh}$ & 8 & 25 & 12 & 3 & 2 & 20 & 33 & 14 & 12 & 9 & 5 & 2 & 25 & 59 & 16 & 42 & 0 & 0 & 18 \\
\hline Haroa Farm $40 \mathrm{C}$ & 9 & 26 & 20 & 3 & 2 & 17 & 32 & 12 & 10 & 9 & 7 & 8 & 22 & 54 & 24 & 42 & 0 & 0 & 12 \\
\hline Haroa Farm $50 \mathrm{C}$ & 10 & 26 & 13 & 2 & 1 & 16 & 28 & 25 & 14 & 9 & 3 & 1 & 19 & 68 & 13 & 42 & 0 & 0 & 15 \\
\hline Haroa Farm $65 \mathrm{C}$ & 11 & 42 & 57 & 16 & 5 & 16 & 23 & 9 & 9 & 8 & 5 & 9 & 37 & 41 & 22 & 42 & 1315 & 0 & 26 \\
\hline Haroa Farm 80 B & 12 & 29 & 3 & 1 & 2 & 15 & 30 & 11 & 14 & 11 & 5 & 10 & 18 & 55 & 27 & 42 & 4 & 0 & 10 \\
\hline \multicolumn{20}{|c|}{ Paleosol in Loessial Apron of tributary of Nahal HaRo'a: Cambic Calcisol (Siltic) } \\
\hline NH-LA-10cm & 5 & 28 & 10 & 3 & 3 & 25 & 39 & 10 & 8 & 7 & 4 & 0 & 32 & 57 & 11 & 42 & 0 & 0 & 27 \\
\hline NH-LA-30cm & 6 & 27 & 5 & 2 & 2 & 23 & 35 & 12 & 10 & 8 & 6 & 1 & 27 & 58 & 15 & 42 & 0 & 0 & 20 \\
\hline \multicolumn{20}{|c|}{ Cistern Cleanout Spoil: Protic Calcaric Regosol (Siltic, Transportic) over Calcaric Regosol (Siltic, Transportic) over Camic Calcisol (Siltic) } \\
\hline HH-WW-Cistern1-Cleanout1-40 & $0 \quad 18$ & 33 & 0 & 0 & 1 & 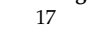 & 23 & 16 & 15 & 11 & 7 & 10 & 18 & 54 & 28 & 42 & 0 & 0 & 8 \\
\hline HH-WW-Cistern1-Cleanout1-80 & $0 \quad 19$ & 34 & 0 & 0 & 1 & 12 & 22 & 16 & 16 & 12 & 8 & 13 & 13 & 54 & 33 & 42 & 4 & 0 & 5 \\
\hline HH-WW-Cistern1-Cleanout1-120 & 2020 & 37 & 2 & 4 & 4 & 22 & 21 & 14 & 12 & 9 & 6 & 9 & 29 & 46 & 24 & 132 & 0 & 0 & 12 \\
\hline HH-WW-Cistern1-Cleanout1-160 & $\begin{array}{ll}60 & 21 \\
\end{array}$ & 38 & 4 & 3 & 5 & 25 & 25 & 12 & 10 & 8 & 6 & 6 & 33 & 48 & 20 & 42 & 0 & 0 & 16 \\
\hline \multicolumn{20}{|c|}{ Hilltop Ruin Overlooking the Western Wadi: Calcaric Leptosol (Protic, Siltic) } \\
\hline HH-WW R1 10 & 22 & 35 & 21 & 4 & 8 & 29 & 18 & 15 & 13 & 9 & 2 & 2 & 42 & 46 & 13 & 132 & 0 & 0 & 25 \\
\hline HH-WW-R1 20 & 23 & 35 & 16 & 5 & 11 & 30 & 12 & 16 & 13 & 8 & 3 & 2 & 45 & 42 & 13 & 132 & 13 & 0 & 28 \\
\hline \multicolumn{20}{|c|}{ Hilltop Ruin Overlooking the Central Wadi: Protic Calcaric Regosol (Siltic) over Cambic Calcisol (Siltic) } \\
\hline HH-CW-Ruin 10 & 24 & 40 & 4 & 2 & 3 & 16 & 22 & 14 & 16 & 14 & 6 & 5 & 22 & 53 & 26 & 42 & 4 & 0 & 10 \\
\hline HH-CW-Ruin 25 & 25 & 42 & 19 & 4 & 3 & 17 & 18 & 16 & 19 & 16 & 5 & 3 & 23 & 53 & 24 & 4 & 42 & 1315 & 11 \\
\hline HH-CW-Ruin 50 & 26 & 40 & 9 & 3 & 6 & 32 & 20 & 14 & 13 & 7 & 3 & 2 & 41 & 47 & 12 & 132 & 0 & 0 & 26 \\
\hline HH-CW-Ruin 60 & 27 & 32 & 24 & 5 & 5 & 27 & 19 & 18 & 14 & 9 & 3 & 1 & $\begin{array}{l}\text { ti } \\
37 \\
\end{array}$ & 51 & 13 & 132 & 0 & 0 & 22 \\
\hline HH-CW-Ruin 75 & 28 & 36 & 12 & 1 & 4 & 15 & 28 & 9 & 14 & 11 & 7 & 11 & 20 & 51 & 29 & 42 & 4 & 0 & 8 \\
\hline HH-CW-Ruin 90 & 29 & 27 & 10 & 2 & 2 & 23 & 22 & 14 & 14 & 11 & 7 & 5 & 27 & 50 & 22 & 132 & 4 & 0 & 13 \\
\hline \multicolumn{20}{|c|}{ Western Wadi Terrace 3: Protic Colluvic Calcaric Regosol (Siltic) } \\
\hline HH-WW-T3-20 & 13 & 36 & 5 & 3 & 4 & 26 & 16 & 14 & 15 & 11 & 6 & 5 & 33 & 44 & 22 & 132 & 4 & 0 & 14 \\
\hline HH-WW-T3-40 & 14 & 43 & 9 & 5 & 6 & 28 & 26 & 12 & 10 & 7 & 4 & 3 & 39 & 48 & 14 & 132 & 0 & 0 & 25 \\
\hline HH-WW-T3-70 & 15 & 48 & 2 & 3 & 4 & 24 & 26 & 16 & 13 & 7 & 4 & 4 & 30 & 55 & 15 & 42 & 0 & 0 & 19 \\
\hline HH-WW-T3-90 & 16 & 43 & 1 & 0 & 1 & 17 & 30 & 21 & 16 & 7 & 3 & 3 & 19 & 68 & 14 & 42 & 0 & 0 & 15 \\
\hline HH-WW-T3-120 & 17 & 41 & 0 & 0 & 1 & 13 & 26 & 19 & 16 & 10 & 7 & 9 & 14 & 61 & 26 & 42 & 0 & 0 & 7 \\
\hline \multicolumn{20}{|c|}{ Negev Reference Samples } \\
\hline Haroa Farm - chalk outcrop & 4 & 79 & n.a. & 0 & 1 & 11 & 1 & 7 & 78 & 1 & 0 & 2 & 12 & 86 & 2 & 4 & 0 & 0 & 5 \\
\hline HH-WW-C2-soft limestone & 2 & 93 & $\mathrm{n}$ & 4 & 3 & 8 & 7 & 53 & 20 & 2 & 1 & 1 & 16 & 80 & 4 & 13 & 132 & 0 & 14 \\
\hline HH-WW-C2-hard limestone & 90 & 93 & n.a. & n.a. & n.a. & n.a. & n.a. & n.a. & n.a. & n.a. & n.a. & n.a. & & & n.a. & n.a. & & n.a. & n.a. \\
\hline HH-CW-chalk & 3 & 98 & n.a. & 5 & 9 & 11 & 1 & 0 & 38 & 35 & 0 & 2 & 25 & 39 & 36 & 4 & 132 & 0 & 8 \\
\hline HH-CW-Tur-Paleo & 1 & 49 & 14 & 2 & 6 & 11 & 12 & 16 & 14 & 8 & 9 & 22 & 20 & 42 & 38 & 13 & 0 & 0 & 3 \\
\hline \multicolumn{20}{|c|}{ Current Dust from Midreshet Ben Gurion, Sede Boker } \\
\hline Dust storm 24./25.03.03 (rain) & 30 & 35 & 0 & 0 & 0 & 2 & 39 & n.a. & 44 & 14 & 0 & 0 & & 84 & 14 & 42 & 0 & 0 & 11 \\
\hline Dust storm 11.12.10 & 31 & 38 & 0 & 0 & 1 & 23 & 38 & $\mathrm{n}$ & 18 & 19 & 1 & 0 & 24 & 56 & 20 & 42 & 1 & 0 & 16 \\
\hline Dust storm 12.12.10 & 32 & 38 & 0 & 0 & 0 & 22 & 41 & $\mathrm{n}$ & 18 & 19 & 1 & 0 & 22 & 59 & 19 & 42 & 1 & 0 & 16 \\
\hline Dust storm 29.02.12 & 33 & 34 & 0 & 0 & 0 & 30 & 46 & & 11 & 12 & 0 & 0 & 30 & 58 & 12 & 42 & 1 & 0 & 27 \\
\hline Dust storm & 34 & 30 & 0 & 0 & 1 & 25 & 48 & & 13 & 12 & 0 & 0 & 26 & 61 & 13 & 42 & 1 & 0 & 24 \\
\hline Dust storm 20.12 & 35 & 33 & 0 & 0 & 0 & 23 & 50 & & 14 & 13 & 0 & 0 & 23 & 64 & 13 & 42 & 1 & 0 & 18 \\
\hline Dust storm 22.03 & 36 & 44 & 0 & 0 & 1 & 23 & 44 & n.a. & 16 & 16 & 0 & 0 & 24 & 60 & 16 & 42 & 1 & 0 & 17 \\
\hline Dust storm 10/11.02.15 & 37 & 40 & 0 & 0 & 0 & 22 & 44 & n.a. & 15 & 17 & 1 & 0 & 22 & 60 & 18 & 42 & 1 & 0 & 16 \\
\hline
\end{tabular}


Table A8. Numbers, sample names, and classification of deposit types used for end-member modeling of grain sizes with EMMAgeo, for principal component analysis (PCA), and for statistical modeling of deposit types based on the random forest approach. Sample numbers match those presented in Figure 6, Figure 7, and Figure 13. Background colors refer to assumed deposit types that we attempted to model/predict by the random forest statistical analysis: green for Negev Rock References, red for Negev Pleistocene Paleosols, grey for Terrace Soils, light grey for Cistern Sediments, yellow for Hilltop Ruin Soils, blue for Negev Dust Storm Sediments, brown for Petra Rock References, orange for Petra Natural Sandstone Soils, dark red for Petra Pleistocene Paleosols, light blue for Petra Dust Storm Sediments, and black/no color for silt\&clay (S\&C) fractions of Petra samples. The hard limestone rock (sample no. 90) was not analyzed for grain sizes, and samples 85-90 not included in EMMAgeo and the statistical (random forest) model of deposit types (which was based on grain sizes, element concentrations, respective calculated statistical grain size parameters and element ratios, and magnetic susceptibilities).

\begin{tabular}{|c|c|c|c|c|c|c|c|c|}
\hline No. & Sample Name & Type & No. & Sample Name & Type & No. & Sample Name & Type \\
\hline 1 & HH-CW-Tur-Paleo & Negev Reference & 31 & Dust storm 11.12 .10 & Negev Dust & 61 & Jh site 3330 & Terrace Soil \\
\hline 2 & HH-WW-C2-soft limestone & Negev Reference & 32 & Dust storm 12.12 .10 & Negev Dust & 62 & Jh site 3350 & Terrace Soil \\
\hline 3 & HH-CW-chalk & Negev Reference & 33 & Dust storm 29.02.12 & Negev Dust & 63 & Jh site 3375 & Terrace Soil \\
\hline 4 & Haroa Farm - chalk & Negev Reference & 34 & Dust storm 18.04 .12 & Negev Dust & 64 & Jh site 33100 & Terrace Soil \\
\hline 5 & & & 35 & Dust storm 20.12.12 & Negev Dust & 65 & Jh site 33130 & Terrace Soil \\
\hline 6 & NH-LA-30cm & $\begin{array}{l}\text { Negev Pleistocene } \\
\text { Negen }\end{array}$ & 36 & Dust storm 22.03.13 & Negev Dust & 66 & Jh site 33150 & Terrace Soil \\
\hline 7 & Haroa Farm $10 \mathrm{C}$ & Terrace Soil & 37 & Dust storm 10/11.02.15 & Negev Dust & 67 & $\mathrm{JF}$ site $124 / 15 \mathrm{~cm}$ & Hilltop Ruin \\
\hline 8 & Haroa Farm 28 fAh & Terrace Soil & 38 & JF site $124 / 1$ rock & Petra Reference & 68 & $J F$ site $124 / 115 \mathrm{~cm}$ & Hilltop Ruin \\
\hline 9 & Haroa Farm $40 \mathrm{C}$ & Terrace Soil & 39 & Sandplateau Stein & Petra Reference & 69 & JF site $124 / 125 \mathrm{~cm}$ & Hilltop Ruin \\
\hline 10 & Haroa Farm $50 \mathrm{C}$ & Terrace Soil & 40 & Baja Sandstein & Petra Reference & 70 & Umm Saysaban $5 \mathrm{~cm}$ & Hilltop Ruin \\
\hline 11 & Haroa Farm $65 \mathrm{C}$ & Terrace Soil & 41 & JH Abu Khushayba sandst. & Petra Reference & 71 & Umm Saysaban $10 \mathrm{~cm}$ & Hilltop Ruin \\
\hline 12 & Haroa Farm 80 B & Negev Pleistocene & 42 & Disi Sandstone & Petra Reference & 72 & Jh limestone 0 & Terrace Soil \\
\hline 13 & HH-WW-T3-20 & Terrace Soil & 43 & Um Ishrin Sandstone & Petra Reference & 73 & Jh limestone 20 & Terrace Soil \\
\hline 14 & HH-WW-T3-40 & Terrace Soil & 44 & Jh limestone 60 outcrop & Petra Reference & 74 & Jh limestone 40 & Terrace Soil \\
\hline 15 & HH-WW-T3-70 & Terrace Soil & 45 & Fan Umm Sayhoun & $\begin{array}{l}\text { Petra Natural } \\
\text { Sandstone Soil }\end{array}$ & 75 & Jh limestone 60 & Terrace Soil \\
\hline 16 & HH-WW-T3-90 & Terrace Soil & 46 & Beidha Fan & $\begin{array}{l}\text { Petra Natural } \\
\text { Sandstone Soil }\end{array}$ & 76 & JH-19-12-16 & Petra Dust \\
\hline 17 & HH-WW-T3-120 & Terrace Soil & 47 & Sandplateau 1 & $\begin{array}{l}\text { Petra Natural } \\
\text { Sandstone Soil }\end{array}$ & 77 & $\mathrm{JH}-07-01-17$ & Petra Dust \\
\hline 18 & HH-WW-Cistern1-Cleanout1-40 & Cistern Sediment & 48 & Sandplateau 2 & $\begin{array}{l}\text { Petra Natural } \\
\text { Sandstone Soil }\end{array}$ & 78 & JH-05-08-17 & Petra Dust \\
\hline 19 & HH-WW-Cistern1-Cleanout1-80 & Cistern Sediment & 49 & Sandplateau 3 & $\begin{array}{l}\text { Petra Natural } \\
\text { Sandstone Soil }\end{array}$ & 79 & JH-05-08-17 closed box & Petra Dust \\
\hline 20 & HH-WW-Cistern1-Cleanout1-120 & Cistern Sediment & 50 & Sandplateau 4 & $\begin{array}{l}\text { Petra Natural } \\
\text { Sandstone Soil }\end{array}$ & 80 & Saleh 25-11-16 & Petra Dust \\
\hline 21 & HH-WW-Cistern1-Cleanout1-160 & Cistern Sediment & 51 & Jh Site $210 \mathrm{~cm}$ & $\begin{array}{l}\text { Petra Natural } \\
\text { Sandstone Soil }\end{array}$ & 81 & Saleh 20-06-17 & Petra Dust \\
\hline 22 & HH-WW R1 10 & Hilltop Ruin & 52 & Jh Site $20 \mathrm{~cm}$ & $\begin{array}{l}\text { Petra Natural } \\
\text { Sandstone Soil }\end{array}$ & 82 & Saleh 05-08-17 & Petra Dust \\
\hline 23 & HH-WW-R1 20 & Hilltop Ruin & 53 & Jh Site $230 \mathrm{~cm}$ & $\begin{array}{l}\text { Petra Natural } \\
\text { Sandstone Soil }\end{array}$ & 83 & $\mathrm{JH}-01-03-17$ & Petra Dust \\
\hline 24 & HH-CW-Ruin Soil 10 & Hilltop Ruin & 54 & Jh Site $240 \mathrm{~cm}$ & $\begin{array}{l}\text { Petra Natural } \\
\text { Sandstone Soil }\end{array}$ & 84 & $\mathrm{JH}-15-02-17$ & Petra Dust \\
\hline 25 & HH-CW-Ruin Soil 25 & Hilltop Ruin & 55 & Jh Site $250 \mathrm{~cm}$ & $\begin{array}{l}\text { Petra Natural } \\
\text { Sandstone Soil }\end{array}$ & 85 & JF site $124 / 15 \mathrm{~cm} \mathrm{S \& C}$ & not modeled \\
\hline 26 & HH-CW-Ruin Soil 50 & Hilltop Ruin & 56 & Jh Site $260 \mathrm{~cm}$ & $\begin{array}{l}\text { Petra Natural } \\
\text { Sandstone Soil }\end{array}$ & 86 & Umm Saysaban $10 \mathrm{~cm} \mathrm{S \& C}$ & not modeled \\
\hline 27 & HH-CW-Ruin Soil 60 & Hilltop Ruin & 57 & Jh Site $270 \mathrm{~cm}$ & $\begin{array}{l}\text { Petra Natural } \\
\text { Sandstone Soil }\end{array}$ & 87 & Jh site $33100 \mathrm{~S} \& \mathrm{C}$ & not modeled \\
\hline $\begin{array}{l}28 \\
29\end{array}$ & HH-CW-Kuin Soil 90 & Negev Pleistocene & $\begin{array}{l}58 \\
59\end{array}$ & $\begin{array}{l}\text { Abu Suwwan below nw } 65 \\
\text { Shakarat Mcaid } 1\end{array}$ & $\begin{array}{l}\text { Petra Pleistocene } \\
\text { Petra Pleistcecen }\end{array}$ & $\begin{array}{l}88 \\
89\end{array}$ & $\begin{array}{l}\text { Shakarat Msaid } 1 \mathrm{S \& C} \\
\text { Jh limestone } 60 \mathrm{~S} \& \mathrm{C}\end{array}$ & $\begin{array}{l}\text { not modeled } \\
\text { not modeled }\end{array}$ \\
\hline 29 & $\begin{array}{l}\text { HH-CW-Ruin Soil } 90 \\
\text { Duststorm } 24,250303 \text { (rain) }\end{array}$ & $\begin{array}{l}\text { Negev Pleistocene } \\
\text { Negev Dust }\end{array}$ & 59 & Shakarat Msaid 1 & Petra Pleistocene & 89 & $\begin{array}{l}\text { Jh limestone } 60 \mathrm{~S} \& \mathrm{C} \\
\mathrm{HH} \text {-WWW-har-limestone }\end{array}$ & not modeled \\
\hline 30 & Dust storm 24./25.03.03 (rain) & Negev Dust & 60 & Jh site 3310 & Terrace Soil & 90 & HH-WW-hard-limestone & not modeled \\
\hline
\end{tabular}


Table A9. Element concentrations and Ti/Zr-ratios from the Petra region. LOI is loss on ignition. The sample no. reflects the sample's number used in statistical analyses, ordered according to types of deposits (see Table A8).

\begin{tabular}{|c|c|c|c|c|c|c|c|c|c|c|c|c|c|c|c|c|c|c|c|c|c|c|c|c|c|c|c|}
\hline Sample Name & $\begin{array}{l}\text { Sample } \\
\text { No. }\end{array}$ & $\underset{\%}{\mathrm{SiO}_{2}}$ & $\begin{array}{c}\mathrm{TiO}_{2} \\
\%\end{array}$ & $\underset{\%}{\mathrm{Al}_{2} \mathrm{O}_{3}}$ & $\underset{\%}{\mathrm{Fe}_{2} \mathrm{O}_{3}}$ & $\underset{\%}{\mathrm{MnO}}$ & $\underset{\%}{\mathrm{MgO}}$ & $\begin{array}{c}\mathrm{CaO} \\
\%\end{array}$ & $\underset{\%}{\mathrm{Na}_{2} \mathrm{O}}$ & $\underset{\%}{\mathrm{~K}_{2} \mathrm{O}}$ & $\underset{\%}{\mathrm{P}_{2} \mathrm{O}_{5}}$ & $\begin{array}{c}\text { LOI } \\
\%\end{array}$ & 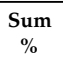 & $\begin{array}{c}\text { Ba } \\
\text { ppm }\end{array}$ & $\begin{array}{c}\mathrm{Cr} \\
\mathrm{ppm}\end{array}$ & $\begin{array}{c}\mathrm{Ga} \\
\mathrm{ppm}\end{array}$ & $\begin{array}{c}\mathrm{Nb} \\
\mathrm{ppm}\end{array}$ & $\begin{array}{c}\mathrm{Ni} \\
\mathrm{ppm}\end{array}$ & $\begin{array}{l}\text { (Pb) } \\
\text { ppm }\end{array}$ & $\begin{array}{c}\mathbf{R b} \\
\mathbf{p p m}\end{array}$ & $\begin{array}{c}\mathrm{Sr} \\
\mathrm{ppm}\end{array}$ & $\begin{array}{c}\text { Th } \\
\text { ppm }\end{array}$ & $\begin{array}{c}\mathrm{V} \\
\mathrm{ppm}\end{array}$ & $\begin{array}{c}\mathrm{Y} \\
\mathrm{ppm}\end{array}$ & $\begin{array}{c}\mathrm{Zn} \\
\mathrm{ppm}\end{array}$ & $\begin{array}{r}\mathrm{Zr} \\
\mathrm{ppm}\end{array}$ & $\mathrm{Ti} / \mathrm{Zr}$ \\
\hline \multicolumn{28}{|c|}{ Natural Hilltop Soil on Sandstone Plateau: Protic Arenosol (Ochric) } \\
\hline Sandplateau 1 & 47 & 89.3 & 0.28 & 3.4 & 0.86 & 0.017 & 0.39 & 1.3 & 0.05 & 0.25 & 0.06 & 3.3 & 99.1 & 86 & 17 & 5.0 & 5.0 & 4.0 & 14 & 7.0 & 104 & 5.0 & 23 & 15 & 21 & 193 & 9 \\
\hline Sandpla & 48 & 91.4 & 0.22 & 2.9 & 0.63 & 0.012 & 0.24 & 0.8 & 0.02 & 0.16 & 0.04 & 2.5 & 98.9 & 53 & 16 & 5.0 & 4.0 & 1.0 & 10 & 5.0 & 88 & 6.0 & 24 & 14 & 16 & 148 & 9 \\
\hline Sandplateau 3 & 49 & 92.9 & 0.23 & 2.9 & 0.62 & 0.011 & 0.20 & 0.3 & 0.02 & 0.15 & 0.04 & 2.0 & 99.4 & 60 & 14 & 4.0 & 4.0 & 1.0 & 15 & 3.0 & 85 & 10.0 & 19 & 15 & 17 & 224 & 6 \\
\hline Sandplateau 4 & 50 & 93.1 & 0.20 & 3.2 & 0.59 & 0.009 & 0.16 & 0.1 & 0.01 & 0.14 & 0.03 & 1.9 & 99.4 & 50 & 14 & 5.0 & 4.0 & 1.0 & 12 & 3.0 & 83 & 11.0 & 22 & 14 & 17 & 149 & 8 \\
\hline Sandplateau Stein (rock) & 39 & 92.9 & 0.09 & 3.7 & 0.10 & 0.003 & 0.02 & 0.1 & 0.01 & 0.03 & 0.07 & 1.6 & 98.6 & 23 & 6 & 5.0 & 3.0 & 1.0 & 11 & 1.0 & 83 & 10.0 & 12 & 12 & 4 & 75 & 7 \\
\hline \multicolumn{28}{|c|}{ Jabal Farasha Triclinium Hilltop Ruin: Calcaric Leptosol (Protic) } \\
\hline IF site $124 / 15 \mathrm{~cm}$ & 67 & 69.1 & 0.60 & 7.2 & 2.46 & 0.036 & 1.26 & 7.5 & 0.54 & 0.77 & 0.22 & 10.1 & 99.8 & 249 & 50 & 7.8 & 13.0 & 15.9 & 17 & 24.3 & 27 & 9.2 & 51 & 24 & 40 & 410 & 9 \\
\hline JF site $124 / 15 \mathrm{~cm} \mathrm{S \& C}$ & 85 & 48.4 & 0.92 & 11.4 & 4.37 & 0.066 & 2.12 & 12.5 & 0.74 & 1.19 & 1.24 & 16.8 & 99.7 & 376 & 76 & 11.3 & 28.3 & 39.3 & 22 & 38.0 & 450 & 11.1 & 84 & 33 & 71 & 541 & 10 \\
\hline JF site & 68 & & & 7.6 & 2. & & & 6.2 & 0.42 & 0. & 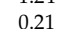 & 8.6 & 99.8 & 191 & 41 & 10.7 & & & & & 277 & & & & 35 & 394 & 9 \\
\hline $\mathrm{JF}$ sit & 69 & 72.9 & 0.61 & 9.0 & 2.42 & 024 & & 5.0 & 0.3 & 0. & 0.2 & 8.1 & 99 & 20 & 48 & 10 & 13 & 16 & 26 & & & & 0 & 4 & 27 & 372 & 10 \\
\hline JF site $124 / 1$ bedrock & 38 & 54.1 & 1.00 & 21.5 & 11.84 & 0.007 & 0.13 & 1.5 & 0.24 & 0.40 & 0.29 & 8.8 & 99.7 & 243 & 113 & 27.4 & 17.0 & 27.5 & 107 & 14.0 & 786 & 18.2 & 92 & 58 & 13 & 182 & 33 \\
\hline \multicolumn{28}{|c|}{ Umm Saysaban Hilltop Ruin: Calcaric Leptosol (Protic) } \\
\hline Umm Saysaban $5 \mathrm{~cm}$ & 70 & 69.1 & 0.60 & 7.9 & 2.31 & 0.034 & 1.12 & 7.6 & 0.44 & 0.58 & 0.23 & 9.9 & 99.8 & 193 & 50 & 13.6 & 13.3 & 23.6 & 22 & 20.4 & 305 & 10.2 & 50 & 23 & 41 & 411 & 9 \\
\hline Umm Saysaban $10 \mathrm{~cm}$ & 71 & 69.5 & 0.61 & 8.2 & 2.11 & 0.028 & 0.96 & 7.5 & 0.58 & 0.50 & 0.22 & 9.6 & 99.8 & 183 & 41 & 9.2 & 13.8 & 12.5 & 24 & 17.3 & 325 & 9.8 & 47 & 24 & 35 & 422 & 9 \\
\hline Umm Saysaban $10 \mathrm{~cm} \mathrm{S \& c}$ & 86 & 44.9 & 0.88 & 13.9 & 3.25 & 0.052 & 1.96 & 13.9 & 0.59 & 0.94 & 1.13 & 18.2 & 99.8 & 304 & 75 & 16.3 & 26.8 & 24.5 & 23 & 32.1 & 493 & 13.3 & 73 & 34 & 65 & 503 & 11 \\
\hline \multicolumn{28}{|c|}{ Buried Early Holocene Paleoso } \\
\hline Shakarat Msaid 1 & 59 & 72.1 & 0.45 & 4.5 & 2.03 & 0.034 & 1.24 & 8.6 & 0.54 & 0.74 & 0.14 & 9.5 & 99.9 & 138 & 44 & 5.5 & 10.3 & 10.3 & 9 & 19.5 & 159 & 6.9 & 47 & 18 & 29 & 312 & 9 \\
\hline Shakarat M & 88 & 45.0 & 0.78 & 9.0 & 3.73 & 0.066 & 2.33 & 16.9 & 0.73 & 1.28 & 1.18 & 18.7 & 99.8 & 306 & 81 & 11.1 & 27.2 & 34.0 & 6 & 32.4 & 300 & 8.1 & 73 & 27 & 63 & 405 & 12 \\
\hline Abu Suwwan below nw 65 & 58 & 71.7 & 0.34 & 3.5 & 1.49 & 0.017 & 1.10 & 10.3 & 0.32 & 0.28 & 0.17 & 10.6 & 99.8 & 127 & 29 & 5.0 & 8.4 & 10.0 & 9 & 7.8 & 483 & 7.0 & 34 & 14 & 29 & 285 & 7 \\
\hline \multicolumn{28}{|c|}{ Monastery Garden of Jabal Haroun: Protic Arenosol (Alcalic. Ochric) } \\
\hline Th Site $210 \mathrm{~cm}$ & 51 & & & 1.8 & 1.21 & 0.015 & 0.43 & & & & & & & 76 & & & & & 8 & & & & & & & & 5 \\
\hline & & & & & & & & 2.2 & & & & & & 90 & & & & & $\begin{array}{l}\circ \\
5\end{array}$ & & & & & & & & 4 \\
\hline & & & & & & & & & & & & & & & & & & & 10 & & & & & & & & 4 \\
\hline & 5 & 87 & & 2. & & & & & & & & & & & & & & & & & & & & & & 431 & 4 \\
\hline Jh Site $260 \mathrm{c}$ & 56 & 88.6 & 0.30 & 2.3 & 1. & & 0.37 & & & & & 3.7 & 99.9 & $1 \mathrm{c}$ & 29 & 5.8 & 7.7 & 15. & 9 & & & 6.3 & & 15 & 25 & 431 & 4 \\
\hline Jh Site $270 \mathrm{~cm}$ & 57 & 84.8 & 0.37 & 3.0 & 2.33 & 0.031 & 0.51 & 3.1 & 0.56 & 0.39 & 0.11 & 4.7 & 99.8 & 150 & 39 & 7.2 & 9.0 & 25.3 & 11 & 12.6 & 182 & 7.4 & 41 & 21 & 33 & 491 & 5 \\
\hline \multicolumn{28}{|c|}{ Terrace on Dolomitic Limestone (Jh site 60): Protic Colluvic Calcaric Regosol } \\
\hline & 72 & 33.8 & 0.3 & 4.1 & 1.9 & 0.033 & 5.18 & 25.3 & 0.34 & 0.66 & 0.28 & 27.9 & 99.9 & 210 & 49 & 5.6 & 8.8 & 8.0 & 5 & 17 & 344 & 3.9 & 49 & 14 & 41 & 248 & 9 \\
\hline & 73 & & & 4.8 & & & 5.38 & 24 & & & & 27 & 99 & 23 & 53 & 6.5 & 9.1 & 10.2 & 4 & 20 & 342 & 4.8 & 53 & 15 & 41 & 241 & 10 \\
\hline $\mathrm{Jl}$ & 74 & 31.5 & 0.36 & 4.1 & 2.06 & 0.030 & 5.48 & 26.3 & & & $0.2-2$ & 28.9 & 99.9 & 214 & 46 & 4.2 & 9.2 & 9.4 & 7 & 16 & 340 & 4.1 & 49 & 14 & 33 & 241 & 9 \\
\hline Jh lime & 75 & 34.0 & 0.40 & 4.4 & 2.14 & 0.032 & 4.97 & 25.2 & 0.3 & 0. & 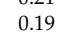 & 27.6 & 99.9 & 210 & 46 & 2.1 & 8.4 & 6.7 & 5 & 15.5 & 335 & $\begin{array}{l}7.1 \\
6.0\end{array}$ & 50 & 14 & 32 & 250 & 10 \\
\hline Jh limestone $60 \mathrm{~S} \& \mathrm{C}$ & $\begin{array}{l}89 \\
89\end{array}$ & 27.0 & 0.50 & 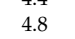 & $\begin{array}{l}2.14 \\
2.42\end{array}$ & 0.038 & 5.27 & 28.5 & 0.40 & 0.66 & 0.55 & 29.7 & 99.8 & 262 & 54 & 4.2 & $\begin{array}{l}0.4 \\
15.4\end{array}$ & 26.7 & 6 & 18.6 & 396 & 6.4 & 51 & 18 & 38 & 279 & 11 \\
\hline \multicolumn{28}{|c|}{ Terrace on Sandstone (Jh site 33): Protic Calcaric Arenosol (Colluvic) over Protic Colluvic Calcaric Regosol } \\
\hline & & & & & 1.67 & & & & & & & & & & & & & & 7 & & & & & & 35 & & 6 \\
\hline & 6 & & & & & & & & & & & & & & & & & & & & & & & & & & 6 \\
\hline & & & & & & & & & & & & & & & & & & & & & & & & & & & 4 \\
\hline & & & & & & & & & & & & & & & 4 & & & & 14 & & & & 55 & 21 & 63 & & $\begin{array}{c}4 \\
10\end{array}$ \\
\hline & 64 & 47 & 0. & 13 & 3. & & 2. & 12 & & 2. & & & 96 & 44 & 52 & & & 26 & 14 & 75 & 35 & 11 & 66 & 23 & 82 & 284 & 15 \\
\hline Jh sit & 87 & 40.5 & 0.8 & 15.3 & 4.32 & 0. & 2.41 & 14. & 0. & 2. & & 18 & 99 & 433 & 54 & 16 & 20 & 31 & 16 & 81 & 41 & 14 & 56 & 27 & 94 & 295 & 18 \\
\hline Jh site 33130 & 65 & 49.1 & 0.56 & 9.9 & 2.98 & 0.030 & 1.88 & 15.4 & 0.4 & 1.60 & 0.19 & 17.8 & 99.8 & 426 & 40 & 12.9 & 12.5 & 16. & 7 & 51.8 & 342 & 10.3 & 57 & 20 & 60 & 306 & 11 \\
\hline Jh site 33150 & 66 & 57.8 & 0.46 & 7.2 & 2.29 & 0.023 & 1.43 & 13.6 & 0.40 & 1.46 & 0.15 & 15.0 & 99.8 & 390 & 39 & 11.5 & 11.1 & 13.2 & 9 & 46.5 & 303 & 10.8 & 44 & 21 & 44 & 403 & 7 \\
\hline
\end{tabular}


Table A9. Cont.

\begin{tabular}{|c|c|c|c|c|c|c|c|c|c|c|c|c|c|c|c|c|c|c|c|c|c|c|c|c|c|c|c|}
\hline Sample Name & $\begin{array}{c}\text { Sample } \\
\text { No. }\end{array}$ & $\begin{array}{c}\mathrm{SiO}_{2} \\
\%\end{array}$ & $\begin{array}{c}\mathrm{TiO}_{2} \\
\%\end{array}$ & $\begin{array}{c}\mathrm{Al}_{2} \mathrm{O}_{3} \\
\%\end{array}$ & $\begin{array}{c}\mathrm{Fe}_{2} \mathrm{O}_{3} \\
\% \\
\end{array}$ & $\begin{array}{c}\mathrm{MnO} \\
\%\end{array}$ & $\begin{array}{c}\mathrm{MgO} \\
\%\end{array}$ & $\begin{array}{c}\mathrm{CaO} \\
\% \\
\end{array}$ & $\begin{array}{c}\mathrm{Na}_{2} \mathrm{O} \\
\%\end{array}$ & $\begin{array}{c}\mathrm{K}_{2} \mathrm{O} \\
\% \\
\end{array}$ & $\begin{array}{c}\mathrm{P}_{2} \mathrm{O}_{5} \\
\%\end{array}$ & $\begin{array}{c}\text { LOI } \\
\%\end{array}$ & $\begin{array}{c}\text { Sum } \\
\%\end{array}$ & $\begin{array}{c}\mathrm{Ba} \\
\mathrm{ppm}\end{array}$ & $\begin{array}{c}\mathrm{Cr} \\
\mathrm{ppm}\end{array}$ & $\begin{array}{c}\mathrm{Ga} \\
\mathrm{ppm}\end{array}$ & $\begin{array}{c}\mathrm{Nb} \\
\mathrm{ppm}\end{array}$ & $\begin{array}{c}\mathrm{Ni} \\
\text { ppm }\end{array}$ & $\begin{array}{l}(\mathrm{Pb}) \\
\mathrm{ppm}\end{array}$ & $\begin{array}{c}\mathbf{R b} \\
\text { ppm }\end{array}$ & $\begin{array}{c}\mathrm{Sr} \\
\text { ppm }\end{array}$ & $\begin{array}{c}\text { Th } \\
\text { ppm }\end{array}$ & $\begin{array}{c}\mathbf{V} \\
\text { ppm }\end{array}$ & $\begin{array}{c}\mathrm{Y} \\
\mathrm{ppm}\end{array}$ & $\begin{array}{c}\mathrm{Zn} \\
\text { ppm }\end{array}$ & $\begin{array}{c}\mathrm{Zr} \\
\mathrm{ppm}\end{array}$ & $\mathrm{Ti} / \mathrm{Zr}$ \\
\hline \multicolumn{28}{|c|}{ Reference Samples from Petra Region: Rocks \& Current Fans } \\
\hline Jh limestone 60 outcrop & 44 & 3.1 & 0.03 & 0.7 & 0.29 & 0.013 & 18.35 & 31.9 & 0.01 & 0.02 & 0.07 & 45.5 & 100.0 & 61 & 16 & 2.3 & 1.2 & 0.5 & 1 & 0.5 & 236 & 2.0 & 21 & 1 & 7 & 11 & 18 \\
\hline Beid & 46 & 71.3 & & 2.9 & & 0.032 & 1.06 & 11.4 & 0.52 & 0.57 & 0.32 & 9.8 & 99.9 & 196 & 48 & 8.8 & & 28 & 8 & 18.5 & & & 40 & 19 & 42 & 370 & 5 \\
\hline Fan umm Sayhoun & 45 & 88.8 & 0.14 & 1.7 & 0.57 & 0.007 & 0.34 & 4.3 & 0.63 & 0.08 & 0.23 & 3.0 & 99.9 & 82 & 38 & 5.9 & 4.9 & 36.8 & 8 & 2.8 & 78 & 2.3 & 24 & 8 & 21 & 213 & 4 \\
\hline $\mathrm{JH}$ sandstone & 41 & 92.1 & 0.14 & 1.5 & 3.52 & 0.012 & 0.05 & 0.6 & 0.52 & 0.04 & 0.11 & 1.3 & 99.9 & 45 & 29 & 4.2 & 4.4 & 21.1 & 26 & 2.3 & 403 & 1.6 & 46 & 9 & 17 & 108 & 8 \\
\hline Disi Sandstone & 42 & 92.9 & 0.13 & 2.0 & 0.14 & 0.001 & 0.20 & 1.4 & 0.37 & 0.03 & 0.07 & 2.7 & 99.9 & 39 & 13 & 3.4 & 3.3 & 7.9 & 5 & 1.7 & 237 & 5.2 & 21 & 6 & 7 & 88 & 6 \\
\hline Um Ishrin & 43 & 93.9 & 0.10 & 0.9 & 1.15 & 0.004 & 0.18 & 1.3 & 0.33 & 0.04 & 0.10 & 1.9 & 99.9 & 58 & 12 & 1.7 & 3.3 & 9.8 & 13 & 1.7 & 306 & 3.7 & 16 & 7 & 8 & 102 & 9 \\
\hline Ba'ja Sandstin & 40 & 77.4 & 0.67 & 12.8 & 0.21 & 0.013 & 0.05 & 1.1 & 0.01 & 0.16 & 0.12 & 5.5 & 98.1 & 67 & 43 & 16.0 & 13.0 & 1.0 & 23 & 2.0 & 507 & 30.0 & 41 & 32 & 14 & 360 & 11 \\
\hline \multicolumn{28}{|c|}{ Mountaintop dust Petra region } \\
\hline JH-19-12-16 & 76 & 91.4 & 0.39 & 1.3 & 0.61 & 0.004 & 0.24 & 1.5 & 0.52 & 0.09 & 0.10 & 3.8 & 99.9 & 94 & 21 & 2.9 & 6.3 & 16.6 & 7 & 2.8 & 110 & 5.5 & 16 & 9 & 58 & 390 & 6 \\
\hline$-07-01-17$ & & 88.2 & 0.25 & 1.7 & 1.23 & & 0.39 & 3.2 & & 0.18 & 0.25 & 3.9 & & 74 & 25 & 7.8 & 6.4 & 22.3 & 13 & & & & 26 & 10 & 47 & 264 & 6 \\
\hline $\mathrm{JH}-01-03$ & 8 & 73 & 0. & 3 & 1. & 0.6 & 0. & 6 & & & & 11.3 & & 1 & & & & & 14 & 14.0 & & & & & 69 & & 10 \\
\hline $\mathrm{JH}-05-08-17$ & 78 & 89.8 & 0.25 & 1.3 & & 0.014 & 0.36 & 2.7 & & 0.21 & & 3.7 & & & & & & & & & & & 22 & 13 & 23 & 369 & 4 \\
\hline JH-05-08-17 closed box & 79 & 89.6 & 0.24 & 1.7 & 1.02 & 0.013 & 0.35 & 2.3 & 0.50 & 0.19 & 0.18 & 3.8 & 99.9 & 96 & 22 & 0.5 & 5.1 & 16.0 & 12 & 5.3 & 112 & 5.5 & 26 & 10 & 25 & 276 & 5 \\
\hline \multicolumn{28}{|c|}{ Foot of mountain dust Petra region } \\
\hline & 80 & 93 & 0. & 0 & 0.8 & 0.005 & 0.11 & 1.3 & 0.34 & 0.09 & & 2.8 & 99.9 & 6 & 15 & 1.0 & & & 6 & & & & 10 & & 01 & 234 & 5 \\
\hline & 81 & 75 & 0 & 2.9 & 1.54 & & 0.79 & 6 & 0. & 0. & 0. & 10.3 & 94 & 1 & 33 & 5.3 & 10.1 & 15.3 & 12 & 11. & 255 & 7.5 & 33 & 15 & 162 & 473 & 7 \\
\hline Saleh 05-08-17 & 82 & 75.4 & 0.53 & 2.9 & 1.55 & 0.019 & 0.76 & 6.4 & 0.52 & 0.43 & 0.42 & 11.0 & 99.8 & 166 & 34 & 1.8 & 9.2 & 8.3 & 12 & 11.9 & 257 & 7.9 & 33 & 17 & 147 & 440 & 7 \\
\hline
\end{tabular}


Table A10. Element concentrations and Ti/Zr-ratios from the Northern Negev. LOI is loss on ignition. The sample no. reflects the sample's number used in statistical analyses, ordered according to types of deposits (see Table A8).

\begin{tabular}{|c|c|c|c|c|c|c|c|c|c|c|c|c|c|c|c|c|c|c|c|c|c|c|c|c|c|c|c|}
\hline Sample Name & $\begin{array}{l}\text { Sample } \\
\text { No. }\end{array}$ & $\underset{\%}{\mathrm{SiO}_{2}}$ & $\begin{array}{c}\mathrm{TiO}_{2} \\
\%\end{array}$ & $\underset{\%}{\mathrm{Al}_{2} \mathrm{O}_{3}}$ & $\begin{array}{c}\mathrm{Fe}_{2} \mathrm{O}_{3} \\
\%\end{array}$ & $\underset{\%}{\mathrm{MnO}}$ & $\underset{\%}{\mathrm{MgO}}$ & $\begin{array}{c}\mathrm{CaO} \\
\%\end{array}$ & $\begin{array}{c}\mathrm{Na}_{2} \mathrm{O} \\
\end{array}$ & $\begin{array}{c}\mathrm{K}_{2} \mathrm{O} \\
\%\end{array}$ & $\begin{array}{c}\mathrm{P}_{2} \mathrm{O}_{5} \\
\%\end{array}$ & $\begin{array}{c}\text { LOI } \\
\%\end{array}$ & $\underset{\%}{S}$ & $\begin{array}{c}\text { Ba } \\
\text { ppm }\end{array}$ & $\underset{\mathrm{ppm}}{\mathrm{Cr}}$ & $\begin{array}{c}\mathrm{Ga} \\
\text { ppm }\end{array}$ & $\begin{array}{c}\mathrm{Nb} \\
\mathrm{ppm}\end{array}$ & $\begin{array}{c}\mathrm{Ni} \\
\mathrm{ppm}\end{array}$ & $\begin{array}{l}\text { (Pb) } \\
\text { ppm }\end{array}$ & $\begin{array}{c}\mathbf{R b} \\
\mathbf{p p m}\end{array}$ & $\begin{array}{c}\mathrm{Sr} \\
\text { ppm }\end{array}$ & $\begin{array}{c}\text { Th } \\
\text { ppm }\end{array}$ & $\begin{array}{c}\mathrm{V} \\
\mathrm{ppm}\end{array}$ & $\begin{array}{c}\mathrm{Y} \\
\mathrm{ppm}\end{array}$ & $\begin{array}{c}\mathrm{Zn} \\
\text { ppm }\end{array}$ & $\begin{array}{c}\mathrm{Zr} \\
\mathrm{ppm}\end{array}$ & Ti//Zr \\
\hline \multicolumn{28}{|c|}{ Terrace on Chalk in Nahal HaRo'a: Protic Colluvic Calcaric Regosol (Siltic) over Calcaric Fluvisol (Aric, Siltic) over Cambic Calcisol (Siltic) } \\
\hline Haroa Farm $10 \mathrm{C}$ & 7 & 49.2 & 0.73 & 6.6 & 3.2 & 0.067 & 2.6 & 14.9 & 1.16 & 1.15 & 0.27 & 19.9 & 99.8 & 357 & 77 & 10.6 & 14 & 18.1 & 5.7 & 30 & 407 & 5.4 & 70 & 21 & 43 & 473 & 9 \\
\hline Haroa Farm 28 fAh & 8 & 50.1 & 0.73 & 6.8 & 3.4 & 0.066 & 2.5 & 16.0 & 1.21 & 0.91 & 0.23 & 17.9 & 99.8 & 331 & 77 & 14.7 & 15 & 24.6 & 7.0 & 23 & 417 & 6.5 & 75 & 23 & 45 & 465 & 9 \\
\hline Haroa Farm $40 \mathrm{C}$ & 9 & 50.4 & 0.74 & 6.8 & 3.4 & 0.065 & 2.5 & 16.4 & 1.22 & 0.93 & 0.22 & 17.1 & 99.8 & 306 & 76 & 7.6 & 15 & 18.7 & 5.0 & 23 & 447 & 6.2 & 69 & 23 & 42 & 458 & 10 \\
\hline Haroa Farm $50 \mathrm{C}$ & 10 & 48.7 & 0.75 & 7.3 & 3.6 & 0.067 & 2.6 & 16.8 & 1.22 & 0.95 & 0.21 & 17.6 & 99.8 & 324 & 77 & 8.3 & 14 & 22.7 & 3.3 & 24 & 486 & 6.6 & 78 & 22 & 44 & 425 & 11 \\
\hline Haroa Farm $65 \mathrm{C}$ & 11 & 38.9 & 0.53 & 5.3 & 2.6 & 0.051 & 2.0 & 25.7 & 0.79 & 0.63 & 0.24 & 23.1 & 99.8 & 262 & 56 & 3.3 & 12 & 16.4 & 5.6 & 16 & 480 & 4.7 & 58 & 19 & 35 & 297 & 11 \\
\hline Haroa Farm 80 B & 12 & 48.4 & 0.95 & 7.5 & 3.9 & 0.075 & 2.4 & 17.6 & 1.21 & 1.06 & 0.23 & 16.4 & 99.8 & 330 & 110 & 9.5 & 17 & 32.2 & 4.5 & 29 & 419 & 6.5 & 94 & 26 & 45 & 536 & 11 \\
\hline \multicolumn{28}{|c|}{ Paleosol in Loessial Apron of tributary of Nahal HaRo'a: Cambic Calcisol (Siltic) } \\
\hline NH-LA-10cm & 5 & 54.3 & 0.89 & 6.2 & 3.2 & 0.062 & 2.0 & 16.1 & 0.95 & 1.19 & 0.20 & 14.6 & 99.8 & 319 & 87 & 10.1 & 15 & 17.3 & 7.2 & 29 & 352 & 8.7 & 74 & 27 & 43 & 713 & 8 \\
\hline NH-LA-30cm & 6 & 53.8 & 0.89 & 6.6 & 3.3 & 0.064 & 2.3 & 15.9 & 0.96 & 1.23 & 0.18 & 14.6 & 99.8 & 337 & 90 & 11.1 & 16 & 21.1 & 6.4 & 30 & 342 & 8.2 & 76 & 24 & 41 & 657 & 8 \\
\hline \multicolumn{28}{|c|}{ Cistern Cleanout Spoil: Protic Calcaric Regosol (Siltic, Transportic) over Calcaric Regosol (Siltic, Transportic) over Camic Calcisol (Siltic) } \\
\hline HH-WW-C.1-Cl.1-40 & 18 & 43.6 & 0.66 & 7.6 & 3.7 & 0.060 & 2.2 & 20.5 & 0.82 & 0.80 & 0.23 & 19.7 & 99.8 & 345 & 76 & 10.9 & 14 & 26.9 & 4.4 & & 367 & 6.4 & 77 & 24 & 51 & 351 & 11 \\
\hline HH-WW-C.1-Cl.1-80 & 19 & 44.0 & 0.67 & 7.7 & 3.7 & 0.063 & 2.2 & 20.3 & 0.83 & 0.82 & 0.23 & 19.4 & 99.8 & 325 & 77 & 8.7 & 13 & 27.1 & 3.8 & 22 & 354 & 5.5 & 75 & 21 & 52 & 337 & 12 \\
\hline HH-WW-C.1-Cl.1-120 & 20 & 44.9 & 0.61 & 6.3 & 2.9 & 0.051 & 1.9 & 21.6 & 0.81 & 0.77 & 0.20 & 19.7 & 99.8 & 336 & 70 & 5.4 & 12 & 17.5 & 5.5 & 20 & 361 & 5.1 & 67 & 20 & 42 & 395 & 9 \\
\hline HH-WW-C.1-Cl.1-160 & 21 & 44.7 & 0.59 & 6.1 & 2.7 & 0.050 & 1.9 & 22.1 & 0.76 & 0.83 & 0.20 & 19.8 & 99.8 & 300 & 65 & 5.7 & 11 & 21.3 & 6.3 & 24 & 367 & 6.5 & 61 & 20 & 38 & 374 & 10 \\
\hline \multicolumn{28}{|c|}{ Hilltop Ruin Overlooking the Western Wadi: Calcaric Leptosol (Protic, Siltic) } \\
\hline HH-WW R1 10 & 22 & 41.8 & 0.63 & 6.0 & 2.6 & 0.048 & 2.2 & 22.7 & 0.64 & 0.65 & 0.21 & 22.3 & 99.8 & 297 & 63 & 5.9 & 14 & 8.9 & 4.6 & 18 & 395 & 5.3 & 62 & 20 & 41 & 432 & 9 \\
\hline HH-WW-R1 20 & 23 & 39.8 & 0.59 & 5.8 & 2.6 & 0.045 & 2.2 & 23.8 & 0.60 & 0.52 & 0.24 & 23.6 & 99.8 & 282 & 62 & 4.2 & 11 & 7.8 & 4.3 & 13 & 418 & 6.8 & 63 & 17 & 38 & 392 & 9 \\
\hline \multicolumn{28}{|c|}{ Hilltop Ruin Overlooking the Central Wadi: Protic Calcaric Regosol (Siltic) over Cambic Calcisol (Siltic) } \\
\hline HH-CW-Ruin 10 & 24 & 39.8 & 0.62 & 6.3 & 3.0 & 0.061 & 2.8 & 23.0 & 0.67 & 1.23 & 0.60 & 21.7 & 99.8 & 326 & 68 & 6.8 & 13 & 20.5 & 6.5 & 31 & 493 & 4.8 & 69 & 20 & 63 & 372 & 10 \\
\hline HH-CW-Ruin 25 & 25 & 38.3 & 0.59 & 5.8 & 2.8 & 0.058 & 2.9 & 24.2 & 0.79 & 0.97 & 0.63 & 22.9 & 99.8 & 373 & 59 & 5.0 & 11 & 12.4 & 7.1 & 23 & 551 & 4.7 & 63 & 17 & 61 & 376 & 9 \\
\hline & 26 & 39.5 & 0.57 & 5.5 & 2.5 & & 3.1 & & & & & & & & 62 & & 12 & & & & & & 55 & 18 & 58 & 68 & 9 \\
\hline & 2 & & 0.68 & 5.6 & 2.5 & & 2.5 & & & & & & & & & & & & & 22 & & & 69 & 18 & 37 & & 8 \\
\hline HH-CW-Ruin 75 & 28 & 44.7 & 0.74 & 6.1 & 3.0 & 0.054 & 2.5 & 21.0 & 0.94 & 1.24 & 0.19 & 19.4 & 99.8 & 299 & 75 & 5.2 & 12 & 15.0 & 7.5 & 26 & 411 & 6.2 & 80 & 22 & 35 & 496 & 9 \\
\hline HH-CW-Ruin 90 & 29 & 48.9 & 0.75 & 6.1 & 2.5 & 0.050 & 2.3 & 18.1 & 0.95 & 0.94 & 0.20 & 19.0 & 99.8 & 295 & 55 & 4.5 & 12 & 9.0 & 10.3 & 22 & 363 & 6.4 & 80 & 18 & 34 & 477 & 9 \\
\hline \multicolumn{28}{|c|}{ Western Wadi Terrace 3: Protic Colluvic Calcaric Regosol (Siltic) } \\
\hline HH-WW-T3-20 & 13 & 45.1 & 0.66 & 6.7 & 3.1 & 0.056 & 1.9 & 21.4 & 0.69 & 1.01 & 0.23 & 18.9 & 99.8 & 338 & 65 & 9.8 & 13 & 17.4 & 8.0 & 30 & 359 & 8.0 & 70 & 23 & 46 & 399 & 10 \\
\hline HH-WW-T3-40 & 14 & 42.1 & 0.59 & 5.4 & 2.5 & 0.048 & 1.8 & 24.2 & 0.67 & 0. & 0.1 & 21 & 99.8 & 3 & 66 & 6.6 & 12 & 17.8 & 5.9 & 21 & 367 & 3.9 & 58 & 17 & 38 & 403 & 9 \\
\hline HH-WW-T3-70 & 15 & 43.2 & 0. & 5.9 & 2.6 & & 2.0 & 23 & 0. & 0. & 0. & 1 & & 3. & 167 & 5.7 & 12 & 98. & 0.8 & 24 & 358 & 3.9 & 58 & 19 & 39 & 369 & 10 \\
\hline HH-WW-T3-90 & 16 & 40.8 & 0.56 & 5.9 & 2.7 & 0.050 & 2.2 & 23.9 & 0.65 & 0.88 & 0.23 & 22.0 & 99.8 & 300 & 56 & 6.5 & 12 & 16.1 & 3.7 & 23 & 350 & 5.1 & 61 & 18 & 40 & 314 & 11 \\
\hline HH-WW-T3-120 & 17 & 40.6 & 0.57 & 6.7 & 3.1 & 0.048 & 2.4 & 23.1 & 0.64 & 0.91 & 0.22 & 21.7 & 99.8 & 300 & 66 & 5.8 & 13 & 19.8 & 6.9 & 25 & 349 & 6.1 & 63 & 19 & 44 & 291 & 12 \\
\hline \multicolumn{28}{|c|}{ Negev Reference Samples } \\
\hline Haroa Farm - chalk & 4 & & & 2.7 & 1.1 & 0.004 & 0.9 & & 0.09 & 0.02 & 0.47 & 39.0 & 99.8 & 335 & 112 & 4.9 & 4 & 39.5 & 4.8 & 0 & 560 & 3.5 & 113 & 16 & 93 & 26 & 33 \\
\hline HH-WW-C2-soft limes & 2 & 0.6 & 0.8 & 0.1 & 0.1 & 0.011 & 0.4 & 55.1 & 0.01 & 0.01 & & 43.5 & & & & 2 & 2 & & 8 & 1 & & 2.8 & 1 & 1 & 6 & 8 & 22 \\
\hline HH-WW-C2-hard limestone & 90 & 0.4 & 0.03 & 0.0 & 0.1 & 0.004 & 0.5 & 55.2 & 0.01 & 0. & 0 & 43 & 10 & 42 & & & 1 & & & 1 & 1 & & 21 & 1 & 6 & 13 & 12 \\
\hline & 3 & 1.1 & 0.03 & 0.2 & 0.2 & & 0.6 & 54 & 0. & 0. & & 43 & 99 & 135 & 1 & & 3 & & & 1 & & & & 1 & 5 & 15 & 14 \\
\hline HH-CW-Tur-Paleo & 1 & 34.3 & 0.56 & 8.8 & 3.7 & 0.020 & 2.1 & 25.1 & 0.51 & 1.09 & 0.17 & 23.6 & 99.8 & 404 & 66 & 9.9 & 10 & 32.6 & 9.7 & 47 & 241 & 8.8 & 115 & 14 & 28 & 212 & 16 \\
\hline \multirow{2}{*}{\multicolumn{28}{|c|}{ Current Dust from Midreshet Ben Gurion, Sede Boker }} \\
\hline dust storm 24./25.03.03 (rain) & 30 & 39.1 & 0.62 & 9.1 & 4.5 & 0.069 & 3.7 & 19.9 & & 1.09 & & & & 305 & 66 & 10.2 & 16 & & 7. & 34 & & 8.3 & 87 & 23 & 78 & 169 & 22 \\
\hline & 31 & & & 6 & 2 & & 2 & 27 & & & & 22.8 & & & 76 & & 12 & & 7.5 & 10 & & 5 & 78 & 21 & 77 & 400 & 10 \\
\hline d & 32 & 42 & & 6.1 & 3.0 & & 2.3 & 27 & & & & 21 & & 46 & 73 & 6.8 & 12 & & 4.1 & 13 & & 6.0 & 71 & 23 & 66 & 410 & 10 \\
\hline t storm 29.02.12 & 33 & 47.5 & 0.67 & 5.7 & 2.7 & 0.052 & 2.3 & 19.4 & 0.86 & 0.7 & 0.2 & 19. & 99 & 427 & 66 & 4.3 & 12 & 17 & 6.2 & 16 & 432 & 4.9 & 59 & 21 & 68 & 489 & 8 \\
\hline st storm 18.04.12 & 34 & 49.3 & 0.75 & 6.6 & 3.1 & 0.059 & 2.5 & 17.0 & 1.02 & 1.04 & 0.24 & 18.1 & 99.8 & 391 & 79 & 8.8 & 14 & 14.2 & 7.1 & 22 & 389 & 6.9 & 75 & 26 & 68 & 502 & 9 \\
\hline ist storm 20.12.12 & 35 & 45.3 & 0.76 & 6.7 & 3.1 & 0.056 & 2.5 & 18.5 & 1.10 & 0.80 & 0.28 & 20.6 & 99.8 & 437 & 82 & 8.4 & 13 & 31.0 & 7.7 & 16 & 454 & 6.0 & 80 & 22 & 76 & 475 & 10 \\
\hline dust storm 22.03.13 & 36 & 38.8 & 0.60 & 5.5 & 2.7 & 0.056 & 2.3 & 24.5 & 0.82 & 0.29 & 0.33 & 23.9 & 99.8 & 461 & 80 & 6.7 & 11 & 31.0 & 3.4 & 7 & 577 & 4.7 & 73 & 22 & 68 & 414 & 9 \\
\hline dust storm 10/11.02.15 & 37 & 38.8 & 0.68 & 6.6 & 3.5 & 0.062 & 3.1 & 22.3 & 0.88 & 0.47 & 0.26 & 23.2 & 99.8 & 486 & 76 & 7.2 & 14 & 25.6 & 6.1 & 11 & 528 & 7.4 & 79 & 27 & 74 & 440 & 9 \\
\hline
\end{tabular}


Table A11. Table summarizing indicators of pedogenesis. Fe(o) is oxalate-extractable iron, Fe(d) dithionite-extractable iron, and Fe(d/t) the ratio of dithionite-extractable to total iron. The Parker-index was calculated according to [66]. $\chi$ is the weight-specific magnetic susceptibility, and $\chi_{\mathrm{FD}}$ the frequency-dependent change of susceptibility. The sample no. reflects the sample's number used in statistical analyses, ordered according to types of deposits (see Table A8).

\begin{tabular}{|c|c|c|c|c|c|c|c|c|c|c|c|}
\hline Sample Name & Sample No. & $\mathrm{Fe}(\mathrm{o})(\mathrm{mg} / \mathrm{g})$ & $\mathrm{Fe}(\mathrm{d})(\mathrm{mg} / \mathrm{g})$ & $\mathrm{Fe}(\mathrm{d} / \mathrm{t})$ & PARKER-Index & $\mathrm{CaCO}_{3} \%$ & Sand \% & Silt \% & Clay \% & $x\left(\mathrm{~m}^{3} / \mathrm{kg}\right) \mathrm{E}-8$ & $\chi_{\mathrm{FD}} \%$ \\
\hline \multicolumn{12}{|c|}{ Petra region } \\
\hline \multicolumn{12}{|c|}{ Natural Hilltop Soil on Sandstone Plateau: Protic Arenosol (Ochric) } \\
\hline Sandplateau 1 & 47 & 0.1 & 2.5 & 0.41 & 7 & 2 & 80 & 12 & 8 & 20.0 & -4.3 \\
\hline Sandplateau 2 & 48 & 0.0 & 2.0 & 0.46 & 4 & 1 & 82 & 9 & 9 & 15.0 & -6.1 \\
\hline Sandplateau 3 & 49 & 0.0 & 2.0 & 0.46 & 3 & 0 & 82 & 9 & 9 & 10.0 & -2.0 \\
\hline Sandplateau 4 & 50 & 0.0 & 1.7 & 0.42 & 2 & 0 & 82 & 8 & 10 & 11.0 & 3.6 \\
\hline Sandplateau Stein (bedrock) & 39 & 0.0 & 0.1 & 0.20 & 1 & 0 & 89 & 7 & 4 & -0.2 & \\
\hline Baja Sandstein (rock) & 40 & 0.0 & 0.2 & 0.15 & 4 & 1 & 72 & 11 & 17 & 0.0 & \\
\hline \multicolumn{12}{|c|}{ Jabal Farasha Triclinium Hilltop Ruin: Calcaric Leptosol (Protic) } \\
\hline$J F$ site $124 / 15 \mathrm{~cm}$ & 67 & 0.2 & 7.5 & 0.44 & 34 & 13 & 51 & 36 & 13 & 32.0 & 5.3 \\
\hline $\mathrm{JF}$ site $124 / 115 \mathrm{~cm}$ & 68 & 0.3 & 6.7 & 0.45 & 28 & 10 & 53 & 35 & 12 & 26.0 & 6.2 \\
\hline JF site 124/1 $25 \mathrm{~cm}$ & 69 & 0.2 & 7.6 & 0.45 & 23 & 8 & 57 & 29 & 14 & 20.0 & 6.5 \\
\hline JF site $124 / 1$ bedrock & 38 & 0.2 & 52.2 & 0.63 & 10 & 2 & 60 & 18 & 22 & 7.0 & 14.4 \\
\hline \multicolumn{12}{|c|}{ Umm Saysaban Hilltop Ruin: Calcaric Leptosol (Protic) } \\
\hline Umm Saysaban $5 \mathrm{~cm}$ & 70 & 0.2 & 5.6 & 0.34 & 31 & 12 & 51 & 35 & 14 & 28.0 & 6.6 \\
\hline Umm Saysaban $10 \mathrm{~cm}$ & 71 & 0.2 & 5.2 & 0.36 & 31 & 12 & 54 & 30 & 16 & 23.0 & 7.0 \\
\hline \multicolumn{12}{|c|}{ Buried Early Holocene Paleosols: Cambic Calcisol (Hypocalcic) (below Shkarat Msaied Ruins); Cambisol (Protocalcic) (below Abu Suwwan Ruins) } \\
\hline Shakarat Msaid 1 & 59 & 0.3 & 4.4 & 0.31 & 36 & 15 & 52 & 26 & 22 & 22.0 & 5.1 \\
\hline Abu Suwwan below nw 65 & 58 & 0.1 & 4.0 & 0.39 & 34 & 18 & 62 & 23 & 15 & 82.0 & 6.3 \\
\hline \multicolumn{12}{|c|}{ Monastery Garden of Jabal Haroun: Protic Arenosol (Alcalic, Ochric) } \\
\hline Jh Site $230 \mathrm{~cm}$ & 53 & 0.1 & 5.0 & 0.43 & 14 & 3 & 80 & 7 & 13 & 15.4 & 18.7 \\
\hline \multicolumn{12}{|c|}{ Terrace on Dolomitic Limestone (Jh site 60): Protic Colluvic Calcaric Regosol } \\
\hline Jh limestone 20 & 73 & 0.3 & 5.0 & 0.33 & 87 & 55 & 32 & 47 & 21 & 28.9 & 1.6 \\
\hline Jh limestone 60 & 75 & 0.2 & 4.8 & 0.32 & 85 & 55 & 30 & 43 & 27 & 24.1 & 1.1 \\
\hline \multicolumn{12}{|c|}{ Terrace on Sandstone (Jh site 33): Protic Calcaric Arenosol (Colluvic) over Protic Colluvic Calcaric Regosol } \\
\hline Jh site 3310 & 51 & 0.2 & 3.9 & 0.34 & 67 & 39 & 66 & 27 & 7 & 20.6 & 1.4 \\
\hline Jh site 3330 & 52 & 0.1 & 4.2 & 0.41 & 38 & 11 & 73 & 15 & 12 & 7.7 & -2.6 \\
\hline Jh site 3350 & 53 & 0.1 & 4.0 & 0.40 & 34 & 7 & 74 & 15 & 11 & 6.3 & -4.8 \\
\hline Jh site 3375 & 54 & 0.1 & 6.8 & 0.33 & 54 & 15 & 32 & 41 & 27 & 16.2 & -0.1 \\
\hline Jh site 33100 & 55 & 0.2 & 10.2 & 0.37 & 60 & 23 & 16 & 45 & 39 & 18.4 & -13.6 \\
\hline Jh site 33130 & 56 & 0.2 & 7.3 & 0.35 & 62 & 28 & 31 & 36 & 33 & 18.9 & 0.9 \\
\hline Jh site 33150 & 57 & 0.2 & 5.9 & 0.37 & 54 & 24 & 59 & 23 & 18 & 15.5 & 0.6 \\
\hline
\end{tabular}


Table A11. Cont

\begin{tabular}{|c|c|c|c|c|c|c|c|c|c|c|c|}
\hline Sample Name & Sample No. & $\mathrm{Fe}(\mathrm{o})(\mathrm{mg} / \mathrm{g})$ & $\mathrm{Fe}(\mathrm{d})(\mathrm{mg} / \mathrm{g})$ & $\mathrm{Fe}(\mathrm{d} / \mathrm{t})$ & PARKER-Index & $\mathrm{CaCO}_{3} \%$ & Sand $\%$ & Silt \% & Clay \% & $\chi\left(m^{3} / k g\right) E-8$ & $\chi_{\mathrm{FD}} \%$ \\
\hline \multicolumn{12}{|c|}{ Negev } \\
\hline \multicolumn{12}{|c|}{ Paleosol in Loessial Apron of tributary of Nahal HaRo'a: Cambic Calcisol (Siltic) } \\
\hline NH-LA-10cm & 5 & 0.4 & 4.6 & 0.20 & 65 & 28 & 32 & 57 & 11 & 58.3 & 2.8 \\
\hline NH-LA-30cm & 6 & 0.4 & 4.6 & 0.20 & 66 & 27 & 27 & 58 & 15 & 61.1 & 2.8 \\
\hline \multicolumn{12}{|c|}{ Western Wadi Terrace 3: Protic Colluvic Calcaric Regosol (Siltic) } \\
\hline HH-WW-T3-20cm & 13 & 0.5 & 5.0 & 0.23 & 74 & 36 & 33 & 45 & 22 & 44.5 & 4.9 \\
\hline HH-WW-T3-70cm & 15 & 0.4 & 4.6 & 0.25 & 80 & 48 & 30 & 55 & 15 & 40.5 & 5.2 \\
\hline \multicolumn{12}{|c|}{ Hilltop Ruin Overlooking the Western Wadi: Calcaric Leptosol (Protic. Siltic) } \\
\hline HH-WW R1 10 & 22 & 0.2 & 2.5 & 0.14 & 75 & 35 & 42 & 45 & 13 & 43.6 & 3.7 \\
\hline HH-WW-R1 20 & 23 & 0.2 & 2.4 & 0.13 & 76 & 35 & 45 & 42 & 13 & 43.7 & 4.5 \\
\hline \multicolumn{12}{|c|}{ Hilltop Ruin Overlooking the Central Wadi: Protic Calcaric Regosol (Siltic) over Cambic Calcisol (Siltic) } \\
\hline HH-CW-Ruin 10 & 24 & 0.5 & 4.3 & 0.20 & 82 & 40 & 22 & 53 & 25 & 64.0 & 6.1 \\
\hline HH-CW-Ruin 25 & 25 & 0.5 & 3.6 & 0.19 & 84 & 42 & 23 & 53 & 24 & 64.0 & 6.9 \\
\hline HH-CW-Ruin 50 & 26 & 0.5 & 3.1 & 0.17 & 85 & 40 & 41 & 47 & 12 & 74.0 & 7.6 \\
\hline HH-CW-Ruin-60 & 27 & 0.2 & 1.8 & 0.11 & 74 & 32 & 37 & 50 & 13 & 60.9 & 4.9 \\
\hline HH-CW-Ruin-75 & 28 & 0.3 & 3.7 & 0.18 & 79 & 36 & 20 & 51 & 29 & 55.0 & 3.0 \\
\hline HH-CW-Ruin-90 & 29 & 0.2 & 2.1 & 0.20 & 69 & 27 & 27 & 51 & 22 & 55.0 & 2.9 \\
\hline
\end{tabular}




\section{Appendix D. Detailed Calculation of Sedimentation Rates}

\section{Appendix D.1. Sedimentation Rates at the Monastery Garden on Jabal Haroun (Jh site 2)}

Due to its position on a sandstone plateau, the profile in the monastery garden was probably not strongly affected by fluvial deposition, and gorges separate the plateau from the surrounding sandstone rocks. However, various high sandstone cliffs showing active Tafoni formation overlook the plateau. Therefore, it seems likely that there is a deposition of sand grains from these rocks by gravity and wind transport. The OSL-age of $150 \mathrm{BCE}-90 \mathrm{CE}$ in $60 \mathrm{~cm}$ matches the pottery from the Roman period found there. Similarly, the ${ }^{14} \mathrm{C}$-age of $239-327 \mathrm{CE}$ in $50 \mathrm{~cm}$ depth corresponds well to the Byzantine material in this layer. Using mean ages and assuming that sediment accumulation continued without interruption until today, one arrives at a deposition of $0.32 \mathrm{~mm} / \mathrm{a}$ during the first 313 years. From then until today, approximately $0.288 \mathrm{~mm} / \mathrm{a}$ were deposited, resulting in a time-normalized average sedimentation of $0.29 \mathrm{~mm} / \mathrm{a}$, or $264 \mathrm{~g} \mathrm{~m}^{-2} \mathrm{a}^{-1}$ of aeolian sediment. This suggests a rather steady and continuous sedimentation, about double as high as on the more isolated hilltop ruin of Jabal Farasha, and more or less identical to the amounts that could annually be collected with the nearby marble dust collectors on Jabal Haroun.

\section{Appendix D.2. Sedimentation Rates at the Terrace on Limestone (Jh site 60)}

The OSL-sample from the bottom of the limestone terrace $(60 \mathrm{~cm}$ depth) showed strong scattering (age range from 2000-27,000 years), which seems connected with a contribution of unbleached grains. This could be due to sample extraction close to the bottom, where older quartz grains from a pre-existing debris layer or other sediment bodies could have been mixed by plowing into the terrace sediment. We, therefore, applied a minimum age model and used only the youngest population of aliquots. The upper sample in $\sim 20 \mathrm{~cm}$ depth also showed some scattering of ages, which could again be connected with the effects of plowing, this time introducing recently bleached grains from the surface. Although effects of current plowing could only be observed up to $10 \mathrm{~cm}$ depth, with rather compact sediment in $20 \mathrm{~cm}$ depth, the age scatter suggests that recent plowing skewed the upper sample to some degree. In addition, the terrace wall had completely filled with sediment, and it is possible that recent land use was connected with some erosion, or at least strongly reduced accumulation of additional material. This means that the top layer of the terrace may consist of a mixture of older and very recent grains.

Using mean ages and assuming continuous sedimentation, one arrives at an average sediment accumulation of $0.2 \mathrm{~mm} / \mathrm{a}$ for the sediment body between the two OSL-ages, and of strongly elevated $1 \mathrm{~mm} / \mathrm{a}$ for the upper $20 \mathrm{~cm}$. Skipping the upper age due to the above mentioned insecurities, one would arrive at $0.27 \mathrm{~mm} / \mathrm{a}$. A similar number would be reached when keeping the age, but normalizing sedimentation rates with respect to the different covered time periods. This suggests a deposition of $\sim 246 \mathrm{~g} \mathrm{~m}^{-2} \mathrm{a}^{-1}$, which is more or less identical to what could be gathered in the dust collectors on Jabal Haroun.

\section{Appendix D.3. Sedimentation Rates at the Cistern Cleanout Spoil}

Although the artificial deposition of the cleanout spoil at the cistern in Horvat Haluqim in the Negev was probably erratic and discontinuous, it is interesting to compare an estimate of average annual deposition with the other archaeological structures. The available ages suggest that a first phase of use dates to the Byzantine and Early Islamic period which lasted around $~ 350$ years, reflected by the now strongly compacted lower part of the cleanout spoil. The upper, friable part of the profile probably refers to a renewed use of the terrace after an interruption of unknown time. Considering the history of the region [14,26] and other ages of similar cistern sediments [59], one could speculate that this re-use took place during the Mamluk and Early Ottoman periods.

Applying the OSL-ages of the dated part of the cleanout spoil, one would arrive at an average sedimentation rate of $1.47 \mathrm{~mm} / \mathrm{a}$, or $1906 \mathrm{~g} \mathrm{~m}^{-2} \mathrm{a}^{-1}$, which exceeds sedimentation in hilltop ruins 
by a factor of $\sim 10-15$, and the material collected in current dust samplers by a factor of $\sim 8$. This amount of sediment is an obvious mismatch to the other structures and clearly reflects its divergent deposition process.

\section{Appendix D.4. Sedimentation Rates at Terrace 3}

Two OSL-ages and one ${ }^{14} \mathrm{C}$-age are available at Terrace 3 . The structure of the profile suggests changes of the deposition process from agricultural use in the lower part, and continued sedimentation without plowing in the upper part. The terrace wall did not yet completely fill with sediment and deposition may continue until today. Applying mean ages, and assuming steady sedimentation, one arrives at a rate of $2.2 \mathrm{~mm} / \mathrm{a}$ of the first $30 \mathrm{~cm}$ until the layer with the charcoal piece. The rate falls to $0.32 \mathrm{~mm} / \mathrm{a}$ for the following $20 \mathrm{~cm}$, and rises to $0.43 \mathrm{~mm} / \mathrm{a}$ for the remaining $70 \mathrm{~cm}$ until the top.

The radiocarbon age does not represent the time of sedimentation, but might have been deposited later, which could explain these strong variations. Using only the OSL-ages, one arrives at average sedimentation rates of $0.67 \mathrm{~mm} / \mathrm{a}$ for the lower $50 \mathrm{~cm}$, and $0.43 \mathrm{~mm} / \mathrm{a}$ for the upper $70 \mathrm{~cm}$. Assuming that no hiati are present, this would result in a time-normalized average sedimentation rate of $0.51 \mathrm{~mm} / \mathrm{a}$, or 453-525 $\mathrm{g} \mathrm{m}^{-2} \mathrm{a}^{-1}$ (considering the change of bulk densities in the upper and lower part of the profile). This is about 3.5 times of the amount that was deposited in hilltop ruins, and double of the amount that accumulated at the limestone terrace in the Petra region.

\section{Appendix D.5. Re-Calculation of Average Annual Sedimentation Rates Applying Revised Bulk Densities}

Lucke et al. [19] estimated that hilltop ruins in both study regions contain more or less exclusively aeolian sediments, and that approximately $0.14 \mathrm{~mm} / \mathrm{a}$ of sediment were deposited in both areas. This resembled around $30 \%$ of the material that could be collected annually with standard marble dust collectors in the Petra region. These values were based on experimentally determined bulk densities of $\sim 1.074 \mathrm{~g} / \mathrm{cm}^{3}$ for hilltop ruins. The hilltop ruin soils contained some small stones and pottery. Although such clasts concentrated largely on the surface, skeleton contents of the hilltop ruin soils could be as high as $2-20 \%$ [19]. As this fraction resembles debris, bulk density of the aeolian fraction of the ruin soil in the triclinium of Jabal Farasha was re-determined to $0.901 \mathrm{~g} / \mathrm{cm}^{3}$ after removing the skeleton content and subtracting its contribution to bulk density, which was measured by water displacement.

In this context, removing the fraction possibly deposited by a dust devil, and separating the two collectors, one would arrive at $41 \mathrm{~g}$ of annual dust collection on the mountaintop of Jabal Haroun, and $38 \mathrm{~g}$ at the foot of the mountain as more conservative estimates. As revision of the calculation presented by Lucke et al. [19], we, therefore, arrive at a minimum of $\sim 250 \mathrm{~g} \mathrm{~m}^{2} \mathrm{a}^{-1}$ dust that can be trapped by standard marble dust collectors. Applying the re-calculated bulk density of only the aeolian fraction, this means that $\sim 125 \mathrm{~g} \mathrm{~m}^{-2} \mathrm{a}^{-1}$ were stored in the hilltop ruin of Jabal Farasha, approximately $50 \%$ of the amount that could be trapped with artificial dust collectors on Jabal Haroun. Taking the one-time event of strong dust deposition on 5 August 2017 into account, possibly by a dust devil, the amount of dust trapped by the collector on the summit of Jabal Haroun would rise to $606 \mathrm{~g} \mathrm{~m}^{2} \mathrm{a}^{-1}$. This would mean that only $20 \%$ were stored in the hilltop soil on Jabal Farasha, but the occurrence of such events is probably too erratic to be included in calculations of average accretion rates.

\section{References}

1. Yaalon, D.H.; Dan, J. Accumulation and distribution of loess-derived deposits in the semi-desert and desert fringe areas of Israel. Z. Geomorphol. Suppl. 1974, 20, e105.

2. Bruins, H.J. The Origin, Nature and Stratigraphy of Paleosols in the Loessial Deposits of the NW-Negev (Netivot, Israel). Master's Thesis, The Hebrew University, Jerusalem, Israel, 1976.

3. Bruins, H.J.; Yaalon, D.H. Stratigraphy of the Netivot section in the desert loess of the Negev (Israel). Acta Geol. Acad. Sci. Hung. 1979, 22, 161-169.

4. Bruins, H.J.; Yaalon, D.H. Parallel advance of slopes in aeolian loess deposits of the northern Negev, Israel. Isr. J. Earth Sci. 1992, 41, 189-199. 
5. Goldberg, P. Late quaternary environmental history of the southern Levant. Geoarchaeology 1986, 1, $225-244$. [CrossRef]

6. Goodfriend, G.A.; Magaritz, M. Palaeosols and late Pleistocene rainfall fluctuations in the Negev Desert. Nature 1988, 332, 144. [CrossRef]

7. Issar, A.S.; Bruins, H.J. Special climatological conditions in the deserts of Sinai and the Negev during the latest Pleistocene, Palaeogeogr. Palaeoclimatol. Palaeoecol. 1983, 43, 63-72. [CrossRef]

8. Bowman, D.; Karnieli, A.; Issar, A.; Bruins, H.-J. Residual colluvio-aeolian aprons in the Negev highlands (Israel) as a palaeo-climatic indicator. Palaeogeogr. Palaeoclimatol. Palaeoecol. 1986, 56, 89-101. [CrossRef]

9. Gerson, R.; Amit, R. Rates and modes of dust accretion and deposition in an arid region: The Negev, Israel. Geol. Soc. Spec. Publ. 1987, 35, 157-169. [CrossRef]

10. Zilberman, E. The Late Pleistocene sequence of the northwestern Negev flood plains-A key to reconstructing the paleoclimate of southern Israel in the last glacial. Isr. J. Earth Sci. 1992, 41, 155-167.

11. Crouvi, O.; Amit, R.; Enzel, Y.; Porat, N.; Sandler, A. Sand dunes as a major proximal dust source for late Pleistocene loess in the Negev Desert, Israel. Quat. Res. 2008, 70, 275-282. [CrossRef]

12. Crouvi, O.; Amit, R.; Enzel, Y.; Gillespie, A.R.; McDonald, E.V. The significance of studying primary hilltop, aeolian loess: An example from the Negev desert, Israel. J. Geophys. Res. 2009, 114, 1-16. [CrossRef]

13. Evenari, M.; Shanan, L.; Tadmor, N. The Negev. In The Challenge of A Desert; Havard University Press: Cambridge, MA, USA, 1971; ISBN 978-0674419247.

14. Avni, G. The Byzantine-Islamic Transition in Palestine: An archaeological approach; Oxford University Press: Oxford, UK, 2014.

15. Al-Qudah, K.; Abdelal, Q.; Hamarneh, C.; Abu-Jaber, N. Taming the torrents: The hydrological impacts of ancient terracing practices in Jordan. J. Hydrol. 2016, 542, 913-922. [CrossRef]

16. Bender, F. Geology of Jordan; Gebrüder Borntraeger: Berlin, Germany, 1974; ISBN 978-3443117078.

17. Cordova, C. Millenial Landscape Change in Jordan; University of Arizona Press: Tucson, USA, 2007; ISBN 978-0816525546.

18. Lucke, B.; Ziadat, F.; Taimeh, A. The Soils of Jordan. In Atlas of Jordan. History, Territories and Society; Ababsa, M., Ed.; Institut Français du Proche-Orient: Beirut, Lebanon, 2013; pp. 72-76.

19. Lucke, B.; Roskin, J.; Vanselow, K.; Bruins, H.; Abu-Jaber, N.; Deckers, K.; Lindauer, S.; Porat, N.; Reimer, P.; Bäumler, R.; et al. Character, rates, and environmental significance of dust accumulation in archaeological hilltop ruins in the southern Levant. Geosciences 2019, 9, 190. [CrossRef]

20. Faershtein, G.; Porat, N.; Avni, Y.; Matmon, A. Aggradation-incision transition in arid environments at the end of the Pleistocene: An example from the Negev Highlands, southern Israel. Geomorphology 2016, 253, 289-304. [CrossRef]

21. Avni, Y.; Porat, N.; Plakht, J.; Avni, G. Geomorphologic changes leading to natural desertification processes versus anthropogenic land conservation in an arid environment, the Negev Highlands, Israel. Geomorphology 2006, 82, 177-200. [CrossRef]

22. Lucke, B. Landscape Transformations in the Context of Soil Development, Land Use, and Climate: A Comparison of Marginal Areas in Jordan, Mexico, and Germany. In Relief Boden Paläoklima 26; Gebrüder Bornträger: Stuttgart, Germany, 2017; ISBN 978-3443090265.

23. Enzel, Y.; Amit, R.; Crouvi, O.; Porat, N. Abrasion-derived sediments under intensified winds at the latest Pleistocene leading edge of the advancing Sinai-Negev erg. Quat. Res. 2010, 74, 121-131. [CrossRef]

24. Swet, N.; Elperin, T.; Kok, J.F.; Martin, R.L.; Yizhaq, H.; Katra, I. Can active sands generate dust particles by wind-induced processes? Earth Planet. Sci. Lett. 2019, 506, 371-380. [CrossRef]

25. Roskin, J.; Katra, I.; Blumberg, D.G. Particle-size fractionation of eolian sand along the Sinai-Negev erg of Egypt and Israel. Geol. Soc. Am. Bull. 2014, 126, 47-65. [CrossRef]

26. Avni, Y.; Avni, G.; Porat, N. A review of the rise and fall of ancient desert runoffagriculture in the Negev Highlands-A model for the southern Levant deserts. J. Arid Environ. 2019, 163, 127-137. [CrossRef]

27. Bruins, H.; Jongmans, A. Micromorphology of ancient agricultural Terraces in the Negev Desert, Horvat Haluqim (Israel). In Proceedings of the 14th International Working Meeting on Soil Micromorphology, Lleida, Spain, 8-14 July 2012; Poch, R.M., Casamitjana, M., Francis, M.L., Eds.; Departament de Medi Ambient i Ciències del Sòl (UdL): Lleida, Spain, 2012; pp. 292-295, ISBN 978-84-615-9132-9.

28. Porat, N.; Avner, U.; Holzer, A.; Shemtov, R.; Horwitz, L.K. Fourth-millennium-BC 'leopard traps' from the Negev Desert (Israel). Antiquity 2013, 87, 714-727. [CrossRef] 
29. Junge, A.; Lomax, J.; Shahack-Gross, R.; Dunseth, Z.C.; Finkelstein, I.; Fuchs, M. OSL Age Determination of archaeological stone structures using trapped aeolian sediments: A Case Study from the Negev Highlands, Israel. Geoarchaeology 2016, 31, 550-563. [CrossRef]

30. Crouvi, O.; Dayan, U.; Amit, R.; Enzel, Y. An Israeli haboob: Sea breeze activating local anthropogenic dust sources in the Negev loess. Aeolian Res. 2017, 24, 39-52. [CrossRef]

31. Bodenheimer, S.; Lensky, I.M.; Dayan, U. Characterization of Eastern Mediterranean dust storms by area of origin; North Africa vs. Arabian Peninsula. Atmos. Environ. 2019, 198, 158-165. [CrossRef]

32. Frösén, J. Petra papyri. In The Encyclopedia of Ancient History; Bagnall, R.S., Brodersen, K., Champion, C.B., Erskine, A., Huebner, S.R., Eds.; Wiley: Hoboken, NJ, USA, 2012. [CrossRef]

33. Lavento, M.; Huotari, M.; Jansson, H.; Silvonen, S.; Fiema, Z. Ancient Water Management System in the Area of Jabal Harûn, Petra. In Men of Dikes and Canals: The Archaeology of Water in the Middle East; Bienert, H., Häser, J., Eds.; Verlag Marie Leidorf: Rahden, Germany, 2004; pp. 163-172. ISBN 978-3896466433.

34. Lavento, M.; Kouki, P.; Silvonen, S.; Ynnilä, H.; Huotari, M. Discussions on terrace cultivation and its relationship to the city of Petra, in southern Jordan. In Studies in the History and Archaeology of Jordan 9; Department of Antiquities: Amman, Jordan, 2007; pp. 145-156.

35. Kouki, P. Archaeological evidence of land tenure in the Petra region, Jordan: Nabataean-Early Roman to Late Byzantine. J. Mediterr. Archaeol. 2009, 22, 29-56. [CrossRef]

36. Kouki, P. The Hinterland of a City. Rural Settlement and Land Use in the Petra Region from the Nabatean-Roman to the Early Islamic Periods; Helsinki University Print: Helsinki, Finland, 2012; ISBN 978-952-10-8015-9.

37. Kouki, P. Appendix 1. Sedimentological studies in the FJHP Survey. In Petra-the Mountain of Aaron III. The Archaeological Survey; Kouki, P., Lavento, M., Eds.; Societas Scientiarum Fennica: Helsinki, Finland, 2013; pp. 44-50. ISBN 9789516534001.

38. Kouki, P.; Lavento, M. The Archaeological Survey. In Petra-the Mountain of Aaron: The Finnish Archaeological Project in Jordan; Societas Scientiarum Fennica: Helsinki, Finland, 2013; Volume 3, ISBN 978-951-653-400-1.

39. Barjous, M.O. The Geology or Petra and Wadi Lahyana Area, Map Sheets No. 3050-I and 3050-IV. Bulletin 56; Hashemite Kingdom of Jordan, Natural Resources Authority, Geology Directorate, Geological Mapping Division: Amman, Jordan, 2003.

40. Lucke, B.; Bäumler, R. Soils and Paleosols at Ba'ja. Neo Lithics 2007, 2, 43.

41. Peel, M.C.; Finlayson, B.L.; McMahon, T.A. Updated world map of the KöppenGeiger climate classification. Hydrol. Earth Syst. Sci. Discuss. 2007, 4, 439-473. [CrossRef]

42. Rosenan, N. Climate. In Atlas of Israel, 2nd ed.; Amiran, D., Elster, J., Gilead, M., Rosenan, N., Kadmon, N., Paran, U., Eds.; map sheet IV/3; Survey of Israel and Elsevier: Amsterdam, The Netherlands, 1970.

43. National Atlas of Jordan. Part One: Agriculture and Climatology; Royal Geographic Centre of Jordan: Amman, Jordan, 1984.

44. Bruins, H.J. Comparative chronology of climatic and human history in the southern Levant from the late Chalcolithic to the Early Arab Period. In Late Quaternary Chronology E Paleoclimates in the Eastern Mediterranean, Proceedings of the 14th International Radiocarbon Conference, Tucson, AZ, USA, 20-21 May 1991; Refereed Special Edition; Radiocarbon \& the American School of Prehistoric Research, Peabody Museum, Harvard University: Cambridge, MA, USA, 1994; pp. 301-314.

45. Bruins, H.J. Runoff terraces in the Negev Highlands during the Iron Age: Nomads settling down or farmers living in the desert? In On the Fringe of Society: Archaeological and Ethnoarchaeological Perspectives on Pastoral and Agricultural Societies; Van der Steen, E.J., Saidel, B.A., Eds.; British Archaeological Reports (BAR) International Series 1657; Archaeopress: Oxford, UK, 2007; pp. 37-43. ISBN 978-1407300931.

46. Bruins, H.J. Ancient desert agriculture in the Negev and climate-zone boundary changes during average, wet and drought years. J. Arid Environ. 2012, 86, 28-42. [CrossRef]

47. Bruins, H.J.; Van der Plicht, J. Assorting and synchronising archaeological and geological strata with radiocarbon: The Southern Levant in relation to Egypt and Thera. In The Synchronisation of Civilizations in the Eastern Mediterranean in the 2nd millennium BC-II; Bietak, M., Ed.; Austrian Academy of Sciences: Vienna, Austria, 2003; pp. 35-42. ISBN 978-3700129363.

48. Bruins, H.J.; Van der Plicht, J. Desert settlement in the central Negev: First 14C indication of rainwater-harvesting agriculture in the Iron Age. In Radiocarbon and Archaeology, Proceedings of the 4th International Symposium, Oxford, UK, 9-14 April 2002; Higham, T.F.G., Bronk Ramsey, C., Owen, D.C., Eds.; Oxford University, School of Archaeology: Oxford, UK, 2004; pp. 83-98. ISBN 978-0947816650. 
49. Bruins, H.J.; Ore, G. Runoff from loess or bedrock? Hillslope geoarchaeology of ancient runoff farming systems at Horvat Haluqim and Har Eldad in the central Negev desert. Isr. J. Earth Sci. 2009, 57, $231-247$. [CrossRef]

50. Bruins, H.J.; Bithan-Guedj, H.; Svoray, T. GIS-based hydrological modelling to assess runoff yields in ancient-agricultural terraced wadi fields (central Negev desert). J. Arid Environ. 2019, 166, 91-107. [CrossRef]

51. Sandler, A. A Turonian Subaerial Event in Israel: Karst, Sandstone, and Pedogenesis; GSI Bulletin 85; Geological Survey of Israel: Jerusalem, Israel, 1996.

52. WRB. World Reference Base for Soil Resources 2014. Update 2015; World Soil Resources Reports 106; FAO: Rome, Italy, 2015; ISBN 978-92-5-108369-7.

53. Soil Survey Staff. Keys to Soil Taxonomy, 12th ed.; United States Department of Agriculture (USDA) \& Natural Resources Conservation Service (NRCS): Washington, DC, USA, 2014.

54. Blott, S.J.; Pye, K. GRADISTAT: A grain size distribution and statistics package for the analysis of unconsolidated sediments. Earth Surf. Process. Landf. 2001, 26, 1237-1248. [CrossRef]

55. Dietze, E.; Hartmann, K.; Diekmann, B.; IJmker, J.; Lehmkuhl, F.; Opitz, S.; Stauch, G.; Wünnemann, B.; Borchers, A. An end-member algorithm for deciphering modern detrital processes from lake sediments of Lake Donggi Cona, NE Tibetan Plateau, China. Sediment. Geol. 2012, 243, 169-180. [CrossRef]

56. R Core Team, R. A Language and Environment for Statistical Computing; R Foundation for Statistical Computing: Vienna, Austria, 2013; Available online: Ftp://ftp.uvigo.es/CRAN/web/packages/dplR/vignettes/intro-dplR. pdf (accessed on 15 March 2019).

57. Liaw, A.; Wiener, M. Classification and Regression by randomForest. R News 2002, 2, 18-22.

58. Abu-Safat, M. Verwitterung und Hangabtragung im "Nubischen Sandstein" Südjordanien, Erlanger Geographische Arbeiten 49; Fränkische Geographische Gesellschaft: Erlangen, Germany, 1988; ISBN 3920405-65-X.

59. Junge, A.; Lomax, J.; Shahack-Gross, R.; Finkelstein, I.; Fuchs, M. Chronology of an ancient water reservoir and the history of human activity in the Negev Highlands, Israel. Geoarchaeology 2018, 33, 695-707. [CrossRef]

60. Lucke, B.; Birk, J.; Hunt, C.; Laparidou, S.; Keilholz, P.; Kouki, P.; Abu-Jaber, N.; Bäumler, R.; Fiedler, S.; Deckers, K.; et al. The terraces of Petra, Jordan: Environmental archives of a lost agricultural hinterland. Geoarchaeology, submitted.

61. Lucke, B. Demise of the Decapolis. Past and Present Desertification in the Context of Soil Development, Land Use, and Climate; Verlag Dr. Müller: Saarbrücken, Germany, 2008; ISBN 978-3639006131.

62. Haliva-Cohen, A.; Stein, M.; Goldstein, S.L.; Sandler, A.; Starinsky, A. Sources and routes of transport of fine detritus material to the late Quaternary Dead Sea basin. Quat. Sci. Rev. 2012, 50, 55-70. [CrossRef]

63. Sandler, A. Clay distribution over the landscape of Israel: From the hyper-arid to the Mediterranean climate regimes. Catena 2013, 110, 119-132. [CrossRef]

64. Lucke, B.; Kemnitz, H.; Bäumler, R.; Schmidt, M. Red Mediterranean Soils in Jordan: New insights in their origin, genesis, and role as environmental archives. Catena 2014, 112, 4-24. [CrossRef]

65. Erel, Y.; Dayan, U.; Rabi, R.; Rudich, Y.; Stein, M. Trans boundary transport of pollutants by atmospheric mineral dust. Environ. Sci. Technol. 2006, 40, 2996-3005. [CrossRef]

66. Parker, A. An index of weathering for silicate rocks. Geol. Mag. 1970, 107, 501-504. [CrossRef]

67. Cornell, R.; Schwertmann, U. The Iron Oxides, 2nd ed.; Wiley VCH: Weinheim, Germany, 2003; ISBN 978-3527302741.

68. Kronberg, B.I.; Nesbitt, H.W. Quantification of weathering soil chemistry and soil fertility. J. Soil Sci. 1981, 32, 453-459. [CrossRef]

69. Kidron, G.J.; Zohar, M.; Starinsky, A. Spatial distribution of dust deposition within a small drainage basin: Implications for loess deposits in the Negev Desert. Sedimentology 2014, 61, 1908-1922. [CrossRef]

70. Kidron, G.J. Biocrust research: A critical view on eight common hydrological-related paradigms and dubious theses. Ecohydrology 2019, 12, e2061. [CrossRef]

71. Kidron, G.J.; Ying, W.; Starinsky, A.; Herzberg, M. Drought effect on biocrust resilience: High-speed winds result in crust burial and crust rupture and flaking. Sci. Total Environ. 2017, 579, 848-859. [CrossRef] [PubMed]

72. Danin, A.; Ganor, E. Trapping of airborne dust by mosses in the Negev Desert, Israel. Earth Surf. Process. Landf. 1991, 16, 153-162. [CrossRef] 
73. McFadden, L.D.; McDonald, E.V.; Wells, S.G.; Anderson, K.; Quade, J.; Forman, S.L. The vesicular layer and carbonate collars of desert soils and pavements: Formation, age and relation to climate change. Geomorphology 1998, 24, 101-145. [CrossRef]

74. Turk, J.K. Vesicular Horizon Distribution, Properties, and Pedogenic Processes in Deserts of the Western United States. Ph.D. Thesis, UC Riverside, Riverside, CA, USA, 2012. Available online: https://escholarship. org/uc/item/325854wj (accessed on 13 March 2019).

75. Yaalon, D.H.; Ginzbourg, D. Sedimentary characteristics and climatic analysis of easterly dust storms in the Negev (Israel). Sedimentology 1966, 6, 315-332. [CrossRef]

76. Offer, Z.Y.; Azmon, E. Chemistry and mineralogy of four dust storms in the northern Negev Desert, Israel (1988-1992). Sci. Total Environ. 1994, 143, 235-243. [CrossRef]

77. Offer, Z.Y.; Goossens, D. Ten years of aeolian dust dynamics in a desert region (Negev desert, Israel): Analysis of airborne dust concentration, dust accumulation and the high-magnitude dust events. J. Arid Environ. 2001, 47, 211-249. [CrossRef]

78. Ganor, E.; Foner, H.A. Mineral dust concentrations, deposition fluxes and deposition velocities in dust episodes over Israel. J. Geophys. Res. Atmos. 2001, 106, 18431-18437. [CrossRef]

79. Kemnitz, H.; Lucke, B. Quartz grain surfaces-A potential microarchive for sedimentation processes and parent material identification in soils of Jordan. Catena 2019, 176, 209-226. [CrossRef]

80. Kalderon-Asael, B.; Erel, Y.; Sandler, A.; Dayan, U. Mineralogical and chemical characterization of suspended atmospheric particles over the east Mediterranean based on synoptic-scale circulation patterns. Atmos. Environ. 2009, 39, 521-533. [CrossRef]

81. Palchan, D.; Erel, Y.; Stein, M. Geochemical characterization of contemporary fine detritus in the Dead Sea watershed. Chem. Geol. 2018, 494, 30-42. [CrossRef]

82. Singer, A.; Ganor, E.; Dultz, S.; Fischer, W. Dust deposition over the Dead Sea. J. Arid Environ. 2003, 53, 41-59. [CrossRef]

83. Singer, A.; Dultz, S.; Argaman, E. Properties of the non-soluble fractions of suspended dust over the Dead Sea. Atmos. Environ. 2004, 38, 1745-1753. [CrossRef]

84. Yaalon, D.; Ganor, E. East Mediterranean Trajectories of Dust-carrying Storms from the Sahara and Sinai. In Saharan Dust: Mobilization, Transport, Deposition; Morales, C., Ed.; Wiley: Chichester, UK, 1979; pp. 187-193.

85. Ganor, E.; Foner, H.A. The mineralogical and chemical properties and the behaviour of aaeolian Saharan dust over Israel. In The Impact of Desert Dust Across the Mediterranean; Guerzoni, S., Chester, R., Eds.; Kluwer Academic Publishers: Dordrecht, The Netherlands, 1996; pp. 163-172. ISBN 978-0792342946.

86. Kubilay, N.; Nickovic, S.; Moulin, C.; Dulac, F. An illustration of the transport and deposition of mineral dust onto the eastern Mediterranean. Atmos. Environ. 2000, 34, 1293-1303. [CrossRef]

87. Kushelevsky, A.; Shani, G.; Haccoun, A. Effect of meteorologic conditions on total suspended particulate (TSP) levels and elemental concentration of aerosols in a semi-arid zone (Beer-Sheva, Israel). Tellus B Chem. Phys. Meteorol. 1983, 35, 55-64. [CrossRef]

88. Ganor, E.; Foner, H.A.; Brenner, S.; Neeman, E.; Lavi, N. The chemical composition of aerosols settling in Israel following dust storms. Atmos. Environ. Part A Gen. Top. 1991, 25, 2665-2670. [CrossRef]

89. Falkovich, A.H.; Ganor, E.; Levin, Z.; Formenti, P.; Rudich, Y. Chemical and mineralogical analysis of individual mineral dust particles. J. Geophys. Res. Atmos. 2001, 106, 18029-18036. [CrossRef]

90. Mahowald, N.; Albani, S.; Kok, J.F.; Engelstaeder, S.; Scanza, R.; Ward, D.S.; Flanner, M.G. The size distribution of desert dust aerosols and its impact on the Earth system. Aeolian Res. 2014, 15, 53-71. [CrossRef]

91. Jeong, G.Y.; Kim, J.Y.; Seo, J.; Kim, G.M.; Jin, H.C.; Chun, Y. Long-range transport of giant particles in Asian dust identified by physical, mineralogical, and meteorological analysis. Atmos. Chem. Phys. 2014, 14, 505-521. [CrossRef]

92. Kok, J.F.; Ridley, D.A.; Zhou, Q.; Miller, R.L.; Zhao, C.; Heald, C.L.; Ward, D.S.; Albani, S.; Haustein, K. Smaller desert dust cooling effect estimated from analysis of dust size and abundance. Nat. Geosci. 2017, 10, 274-278. [CrossRef]

93. Abed, A.M.; Al Kuisi, M.; Khair, H.A. Characterization of the Khamaseen (spring) dust in Jordan. Atmos. Environ. 2009, 43, 2868-2876. [CrossRef]

94. Bonjour, J.L.; Dabard, M.P. Ti/Nb ratios of clastic terrigenous sediments used as an indicator of provenance. Chem Geol. 1991, 91, 257-267. [CrossRef] 
95. Fiema, Z.T.; Frösén, J. (Eds.) The Church and the Chapel. In Petra-The Mountain of Aaron: The Finnish Archaeological Project in Jordan; Societas Scientiarum Fennica: Helsinki, Finland, 2008; Volume 1, ISBN 978-951-653-364-6.

96. Fiema, Z.; Frösén, J.; Holappa, M. Petra-The mountain of Aaron: The Finnish archaeological project in Jordan. The Nabataean Sanctuary and the Byzantine Monastery; Societas Scientiarum Fennica: Helsinki, Finland, 2016; Volume 2, ISBN 978-951-653-410-0.

97. Shkarat Msaied Neolithic Project. Available online: https://shkaratmsaied.tors.ku.dk/radiocarbondates/ (accessed on 15 October 2019).

98. Silvonen, S.; Kouki, P.; Eklund, A.; Hertell, E.; Lavento, M.; Ynnilä, H. The FJHP Survey Site Catalog. In Petra-The Mountain of Aaron: The Finnish Archaeological Project in Jordan. The Archaeological Survey; Kouki, P., Lavento, M., Eds.; Societas Scientiarum Fennica: Helsinki, Finland, 2013; Volume 3, pp. 347-409.

99. Silvonen, S. Nabatean-Roman, Byzantine, and Early Islamic Pottery from the FJHP Survey. In Petra-the Mountain of Aaron: The Finnish Archaeological Project in Jordan. The Archaeological Survey; Kouki, P., Lavento, M., Eds.; Societas Scientiarum Fennica: Helsinki, Finland, 2013; Volume 3, pp. 120-148.

100. Lavento, M.; Silvonen, S.; Kouki, P. Runoff Cultivation and Hydraulic Structures. In Petra-The mountain of Aaron: The Finnish Archaeological Project in Jordan. The Archaeological Survey; Kouki, P., Lavento, M., Eds.; Societas Scientiarum Fennica: Helsinki, Finland, 2013; Volume 3, pp. 213-230.

101. Cohen, R. The Iron Age fortresses in the central Negev. Bull. Am. Sch. Orient. Res. 1979, 236, 61-79. [CrossRef]

102. Turk, J.; Houdeshell, C.-A.; Graham, R. A Proposed Master V Horizon for the Designation of Near-Surface Horizons with Vesicular Porosity. 2016. Available online: https://www.nrcs.usda.gov/Internet/FSE_ DOCUMENTS/nrcs142p2_050966.pdf (accessed on 13 March 2019).

103. Müller, G.; Gastner, M. The "Karbonat-Bombe", a simple device for the determination of the carbonate content in sediments, soils and other materials. Neues Jahrbuch für Mineralogie 1971, 10, 466-469.

104. Deutsches Institut für Normung. Blatt 1-3, Physikalische Laboruntersuchungen, Bestimmung der Korngrößenzusammensetzung nach Vorbehandlung mit Natriumpyrophosphat; DIN 19683; Beuth Verlag: Berlin, Germany, 1973.

105. Gerzabek, M.H. Die Bestimmung Der Bodentextur Mittels Sedigraph 5100; OEFZS Report No. 4592; Österreichisches Forschungszentrum Seibersdorf: Seibersdorf, Austria, 1991.

106. Schlichting, E.; Blume, H.-P.; Stahr, K. Bodenkundliches Praktikum, 2nd ed.; Blackwell Wissenschafts-Verlag: Berlin, Germany, 1995; ISBN 978-3826330421.

107. Schwertmann, U. Use of oxalate for Fe extraction from soils. Can. J. Soil Sci. 1973, 53, 244-246. [CrossRef]

108. Slota, P.J.; Jull, A.T.; Linick, T.W.; Toolin, L. Preparation of small samples for $14 \mathrm{C}$ accelerator targets by catalytic reduction of CO. Radiocarbon 1987, 29, 303-306. [CrossRef]

109. Stuiver, M.; Polach, H.A. Discussion reporting of 14 C data. Radiocarbon 1977, 19, 355-363. [CrossRef]

110. Murray, A.S.; Wintle, A.G. Luminescence dating of quartz using an improved single-aliquot regenerative-dose protocol. Radiat. Meas. 2000, 32, 57-73. [CrossRef]

111. Duller, G.A.T.; Aberystwyth University, Aberystwyth, UK. Personal communication, 2008.

112. Folk, R.L.; Ward, W.C. Brazos River bar [Texas]; a study in the significance of grain size parameters. J. Sediment. Res. 1957, 27, 3-26. [CrossRef]

113. Reyment, R.A.; Jöreskog, K.G. Applied Factor Analysis in the Natural Sciences, 2nd ed.; Cambridge University Press: Cambridge, UK, 1997; ISBN 978-0521575560.

114. EMMAgeo. End-Member Modelling of Grain-Size Data v0.9.4. Available online: https://www.rdocumentation. org/packages/EMMAgeo/versions/0.9.4 (accessed on 19 February 2019).

115. Dietze, E.; Maussion, F.; Ahlborn, M.; Diekmann, B.; Hartmann, K.; Henkel, K.; Kasper, T.; Lockot, G.; Opitz, S.; Haberzettl, T. Sediment transport processes across the Tibetan Plateau inferred from robust grain-size end members in lake sediments. Clim. Past 2014, 10, 91-106. [CrossRef]

116. Hartmann, K.; Wünnemann, B. Hydrological changes and Holocene climate variations in NW China, inferred from lake sediments of Juyanze palaeolake by factor analyses. Quat. Int. 2009, 194, 28-44. [CrossRef]

117. Manson, V.; Imbrie, J. Kansas Geological Survey Special Distribution Publications. FORTRAN Program for Factor and Vector Analysis of Geological Data Using an IBM 7090 or 7094/1401 Computer System; Kansas Geological Survey: Lawrence, KS, USA, 1964; Volume 13.

118. Klovan, J.E.; Imbrie, J. An algorithm and FORTRAN-IV program for large-scale Q-mode factor analysis and calculation of factor scores. Math. Geol. 1971, 3, 61-77. [CrossRef] 
119. Renner, R.M.; Glasby, G.P.; Szefer, P. Endmember analysis of heavy-metal pollution in surficial sediments from the Gulf of Gdansk and the southern Baltic Sea off Poland. Appl. Geochem. 1998, 13, 313-318. [CrossRef]

120. Kaiser, H.F. The VARIMAX criterion for analytic rotation in factor analysis. Psychometrika 1958, 23, 187-200. [CrossRef]

121. Sachs, L.; Hedderich, J. Angewandte Statistik. Methodensammlung mit R; Springer: Berlin/Heidelberg, Germany, 2006. [CrossRef]

122. Breiman, L. Random forests. Mach. Learn. 2001, 45, 5-32. [CrossRef]

123. Stuiver, M.; Reimer, P.J. Extended C-14 Data-Base and Revised Calib 3.0 C-14 Age Calibration Program. Radiocarbon 1993, 35, 215-230. [CrossRef]

124. Reimer, P.J.; Bard, E.; Bayliss, A.; Beck, J.W.; Blackwell, P.G.; Ramsey, C.B.; Buck, C.E.; Cheng, H.; Edwards, R.L.; Friedrich, M.; et al. IntCal13 and Marine13 radiocarbon age calibration curves $0-50,000$ years cal BP. Radiocarbon 2013, 55, 1869-1887. [CrossRef]

(C) 2019 by the authors. Licensee MDPI, Basel, Switzerland. This article is an open access article distributed under the terms and conditions of the Creative Commons Attribution (CC BY) license (http://creativecommons.org/licenses/by/4.0/). 